\title{
Direct Catalytic Asymmetric Mannich-type Reaction of $\alpha$ - and $\beta$-Fluorinated Amides
}

Lennart Brewitz, Fernando Arteaga Arteaga, Liang Yin, Kaliyamoorthy Alagiri, Naoya Kumagai,"* and Masakatsu Shibasaki*

Institute of Microbial Chemistry (BIKAKEN), Tokyo, Japan

mshibasa@bikaken.or.jp,nkumagai@bikaken.or.jp

1. General

2. Instrumentation

3. Materials

4. General Procedures

4-1. Preparation of amides.

4-2. Preparation of $\mathrm{N}-\mathrm{Boc}$ and $\mathrm{N}-\mathrm{Cbz}$ imines

4-3. Direct catalytic asymmetric Mannich-type reaction of $\alpha-\mathrm{CF}_{3}$ amide $1 \mathrm{a}$.

4-4. Direct catalytic asymmetric Mannich-type reaction of $\alpha-F$ $\alpha-\mathrm{CF}_{3}$ amide $\mathbf{1 b}$.

4-5. Direct catalytic asymmetric Mannich-type reaction of $\alpha-\mathrm{CF}_{2} \mathrm{CF}_{3}$ amide $\mathbf{1 c}$ and $\alpha-\mathrm{CF}_{2} \mathrm{CF}_{2} \mathrm{Br}$ amide $\mathbf{1 d}$.

4-6. Attempted direct catalytic asymmetric Mannich-type reaction of $\alpha-C F\left(\mathrm{CF}_{3}\right)_{2}$ amide $\mathbf{1 f}$.

4-7. Direct catalytic asymmetric Mannich-type reaction of $\alpha-F$ amide 1e. S47-S54

4-8. Transformation of the Mannich products.

5. Determination of the Absolute and Relative Configuration of the Products

6. Chemical Shifts of Fluorinated 7-Azaindoline Amides and Related Compounds

7. Conformational Analysis of Amides $\mathbf{1 a}$ and $\mathbf{1 b}$

8. Preparation and Characterization of $\mathrm{Cu}(\mathrm{I}) / \mathrm{rac}-\mathrm{BINAP} / \mathrm{1a}$ Complex

9. Separation of Amides $(R)-\mathbf{1 b}$ and $(S)-\mathbf{1 b}$ and Determination of the Absolute Configuration

10. HOESY Analysis of $\mathrm{Cu}(\mathrm{I}) /(R)-\mathrm{xyl}-\mathrm{BINAP} /(S)-\mathbf{1 b}$ Complex

11. Ligand Screening for the Mannich-type Reaction of $\alpha-F$ amide 1 e

12. Acute Toxicity of $\alpha-F$ Amide 1e

13. References

14. ${ }^{1} \mathrm{H},{ }^{13} \mathrm{C}$, and ${ }^{19} \mathrm{~F}$ NMR Spectra of the New Compounds (see separate file)
S71

S2

S2

S2

S3-S57

S3-S12

$\mathrm{S} 12$

S12-S28

S28-S38

S38-S46

S47

S54-S57

S58-S60

S61-S63

S63-S66

S66-S68

S69-S70

S71-S72

S72

S73

S73-S240 


\section{General}

All reactions were performed under Ar atmosphere unless otherwise noted. The direct catalytic asymmetric Mannich-type reaction was performed in a $20 \mathrm{~mL}$ glass test tube with a 3-way glass stopcock. Air- and moisture-sensitive liquids were transferred via a gas-tight syringe and a stainless-steel needle. All work-up and purification procedures were carried out with reagent-grade solvents under ambient atmosphere.

\section{Instrumentation}

Infrared (IR) spectra were recorded on a JASCO FT/IR-4100 Fourier transform infrared spectrophotometer. NMR spectra were recorded on JEOL ECS-400 or ECS-600 and Bruker AVANCE III HD 400 or AVANCE III 500. Chemical shifts for protons are reported in parts per million (ppm) downfield from tetramethylsilane and referenced to residual protium in the NMR solvent $\left(\mathrm{CDCl}_{3}: \delta=7.28\right.$ ppm; DMSO- $d_{6}: \delta=2.49$ ppm; MeCN- $d_{3}: \delta=1.94$ ppm). For ${ }^{13} \mathrm{C}$ NMR, chemical shifts are reported in the scale relative to the NMR solvents $\left(\mathrm{CDCl}_{3}: \delta=77.00\right.$ ppm; DMSO- $d_{6}: \delta=39.52$ ppm; MeCN-d $d_{3} \delta=118.26,1.32 \mathrm{ppm}$ ) as an internal reference and for ${ }^{19} \mathrm{~F} \mathrm{NMR}$, chemical shifts are reported in the scale relative to TFA or trifluorotoluene as an external reference $(\delta=-76.5$ ppm respectively $\delta=-62.8$ ppm). NMR data are reported as follows: chemical shift, multiplicity (s: singlet, d: doublet, dd: doublet of doublets, t: triplet, q: quartet, hept.: heptet, m: multiplet, br: broad signal), coupling constant (Hz), and integration. Single-crystal X-ray data were collected on a Rigaku R-AXIS RAPID II imaging plate area detector with graphite-monochromated Cu-K $\alpha$ radiation. High resolution mass spectra (ESI Orbitrap (+)) were measured on ThermoFisher Scientific LTQ Orbitrap XL. Melting points were measured on a Yanagimoto Seisakusho Micro Melting Point Apparatus. Optical rotations were measured using a $2 \mathrm{~mL}$ cell with a $1.0 \mathrm{dm}$ path length on a JASCO polarimeter P-1030. HPLC analysis was conducted on a JASCO HPLC system equipped with Daicel chiral-stationary-phase columns $(0.46 \mathrm{~cm} \phi \times 25 \mathrm{~cm})$.

\section{Materials}

Unless otherwise noted, all materials were purchased from commercial suppliers (Sigma-Aldrich, TCI) and were used without further purification. $\left[\mathrm{Cu}\left(\mathrm{CH}_{3} \mathrm{CN}\right)_{4}\right] \mathrm{PF}_{6}$ and chiral phosphine ligands were purchased from Sigma-Aldrich or Strem Chemicals, Inc. and were stored and handled in a glove box. Barton's base and T3P ${ }^{\circledR}$ solution (50\% solution in ethyl acetate) were obtained from Sigma-Aldrich; 2,3-dihydro-7-azaindole was either purchased (Sigma-Aldrich or Combi-Blocks, Inc.) or prepared from 7-azaindole (purchased from Ark Pharma, Inc. or Combi-Blocks, Inc.) by following literature procedures. ${ }^{1}$ The fluorinated building blocks necessary for amide preparations were purchased from the following companies and used as received: 3,3,3-trifluoropropanoic acid (Apollo Scientific Ltd.); 2,3,3,3-tetrafluoropropanoic acid (Oakwood Products, Inc. or Matrix Scientific Ltd.); 3,3,4,4,4-pentafluorobutan-1-ol (Matrix Scientific Ltd. or Oakwood Products, Inc.); 4-bromo-3,3,4,4-tetrafluorobutan-1-ol (Apollo Scientific Ltd.). THF and diethyl ether were passed through a solvent purification system (Glass Contour). Flash column chromatography was performed on Merck 60 (230-400 mesh) or Kanto Chemical 60N (neutral, spherical, 50-60 $\mu$ m) silica gel. 


\section{General Procedures}

\section{4-1. Preparation of amides.}

\section{1-(2,3-Dihydro-1H-pyrrolo[2,3-b]pyridin-1-yl)-3,3,3-trifluoropropan-1-one (1a).}

In a previous communication of this work, the synthesis of the title compound was reported to start from commercially available 3,3,3-trifluoropropionyl chloride; ${ }^{2}$ however, it was later found that starting from the corresponding cheaper and more stable acid, the title compound can be obtained in higher yield:

2,3-Dihydro-7-azaindole (1.95 g, $16.2 \mathrm{mmol}, 1.05$ equiv.), triethylamine (4.3 mL, 30.8 mmol, 2.0 equiv.) and T3P ${ }^{\circledR}$ solution (12.3 g of a 50\% solution in ethyl acetate, $19.3 \mathrm{mmol}, 1.25$ equiv.) were subsequently added to a solution of 3,3,3-trifluoropropionic acid $\left(1.36 \mathrm{~mL}, 15.4 \mathrm{mmol}, 1.0\right.$ equiv.) in dichloromethane $(75 \mathrm{~mL})$ at $0{ }^{\circ} \mathrm{C}$. The reaction mixture was stirred at ambient temperature for $2 \mathrm{~h}$ before it was diluted with saturated aqueous $\mathrm{NaHCO}_{3}$ solution and extracted three times with dichloromethane; the combined organic extracts were dried over $\mathrm{Na}_{2} \mathrm{SO}_{4}$, filtered and evaporated. The residue was purified by flash column chromatography (hexane/ethyl acetate, 2:1) to yield amide 1a as a white solid $(2.4 \mathrm{~g}, 68 \%)$

White fluffy solid, m. p.: $111-113{ }^{\circ} \mathrm{C} ;{ }^{1} \mathrm{H}$ NMR $\left(400 \mathrm{MHz}, 293 \mathrm{~K}, \mathrm{CDCl}_{3}\right): \delta=8.09$ (d, J=5.3 Hz, $\left.1 \mathrm{H}\right), 7.50(\mathrm{dq}, J=7.4,1.2$ $\mathrm{Hz}, 1 \mathrm{H}), 6.92(\mathrm{dd}, J=7.3,5.0 \mathrm{~Hz}, 1 \mathrm{H}), 4.21(\mathrm{q}, J=10.4 \mathrm{~Hz}, 2 \mathrm{H}), 4.12(\mathrm{t}, J=8.5 \mathrm{~Hz}, 2 \mathrm{H}), 3.08 \mathrm{ppm}(\mathrm{t}, J=8.6 \mathrm{~Hz}, 2 \mathrm{H}) ;{ }^{13} \mathrm{C}$ $\operatorname{NMR}\left(100 \mathrm{MHz}, 293 \mathrm{~K}, \mathrm{CDCl}_{3}\right): \delta=162.4$ (q, J = 3.8 Hz), 155.0, 145.9, 133.9, 126.0, 124.3 (q, J = 276.4 Hz), 118.7, $45.6,40.4$ $(\mathrm{q}, J=28.8 \mathrm{~Hz}), 23.9 \mathrm{ppm} ;{ }^{19} \mathrm{~F} \mathrm{NMR}\left(376 \mathrm{MHz}, 293 \mathrm{~K}, \mathrm{CDCl}_{3}\right): \delta=-62.2 \mathrm{ppm}(\mathrm{t}, J=11.6 \mathrm{~Hz})$; IR (film): $\widetilde{v}=2992,2970,1666$, $1602,1474,1443,1431,1412,1389,1264,1253,1239,1172,1109,848,791 \mathrm{~cm}^{-1}$; HRMS (ESI): m/z calculated for $\mathrm{C}_{10} \mathrm{H}_{9} \mathrm{~F}_{3} \mathrm{~N}_{2} \mathrm{ONa}[\mathrm{M}+\mathrm{Na}]^{+}:$253.0559, found: 253.0555.

In order to determine the conformation of amide 1a in the solid state, a single-crystal was grown by leaving a concentrated solution of 1a in $n$-hexane/ethyl acetate at room temperature. Single-crystal X-ray data were collected on a Rigaku R-AXIS RAPID II imaging plate area detector with graphite-monochromated Cu-K $\alpha$ radiation. Data collection was conducted at $93 \mathrm{~K}$. All structures were solved by direct methods and refined by full matrix least-squares against $F^{2}$ with all reflections. All non-hydrogen atoms were refined anisotropically. All hydrogen atoms were placed in standard calculated positions, and were refined using the riding model. CCDC 1430608 contains the supplementary crystallographic data for 1a. Refined structure and crystallographic parameters are summarized in Figure S1 and Table S1.

The conformation of amide 1a in the solid state was determined to be $E$-conformation, which is expected based on similar literature-known amides.3,4 In Section 7 (page S63-S66), the conformation of 1a was also examined in solution phase: NOE contacts were not observed between the $\alpha$-protons and the protons at the indolinyl group; the ${ }^{1} \mathrm{H}$ NMR spectra of 1a showed the existence of only one conformer at a wide range of temperatures; the addition of TFA- $d_{1}$ resulted in the unambiguous formation of the Z-conformer of 1a. Even though these experiments are not fully conclusive, they strongly suggest that only the E-conformer of 1a is present in the solution phase. 


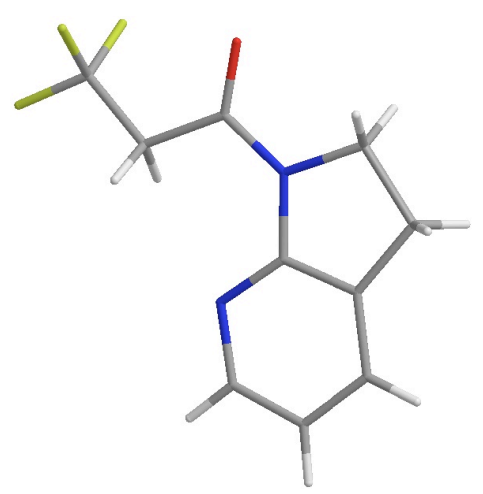

Figure S1. The structure of $1 \mathrm{a}$ in the solid state (E-conformation). Color code: light green: fluorine; red: oxygen; blue: nitrogen; gray: carbon; white: hydrogen.

\begin{tabular}{c|c}
\hline & 1a \\
\hline molecular formula & $\mathrm{C}_{10} \mathrm{H}_{9} \mathrm{~F}_{3} \mathrm{~N}_{2} \mathrm{O}$ \\
formula weight & 230.19 \\
crystal color, habit & colorless, platelet \\
crystal system & monoclinic \\
space group & $P 21 / n$ \\
cell constants: & \\
$a(\AA)$ & $4.69994(14)$ \\
$b(\AA)$ & $9.6731(3)$ \\
$c(\AA)$ & $21.1552(6)$ \\
$\beta(\mathrm{deg})$ & $95.859(7)$ \\
$V\left(\AA{ }^{3}\right)$ & $956.76(5)$ \\
$Z$ & 4 \\
$\rho_{\text {calcd. }}\left(\mathrm{g} \cdot \mathrm{cm}^{-3}\right)$ & 1.598 \\
$R 1$ & 0.0420 \\
$w R 2$ & 0.1131 \\
$F(000)$ & 472.00 \\
&
\end{tabular}

\section{1-(2,3-Dihydro-1H-pyrrolo[2,3-b]pyridin-1-yl)-2,3,3,3-tetrafluoropropan-1-one (1b).}

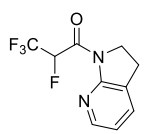

2,3-Dihydro-7-azaindole (1.95 g, $16.2 \mathrm{mmol}, 1.05$ equiv.), triethylamine (4.3 mL, $30.8 \mathrm{mmol}, 2.0$ equiv.) and $\mathrm{T}^{\mathrm{P}} \mathrm{P}^{\circledR}$ solution (12.3 $\mathrm{g}$ of a $50 \%$ solution in ethyl acetate, $19.3 \mathrm{mmol}, 1.25$ equiv.) were subsequently added to a solution of 2,3,3,3-tetrafluoropropionic acid (2.25 g, $15.4 \mathrm{mmol}, 1.0$ equiv.) in dichloromethane (30 $\mathrm{mL})$ at

$0{ }^{\circ} \mathrm{C}$. The reaction mixture was stirred at ambient temperature for $2 \mathrm{~h}$ before it was diluted with saturated aqueous $\mathrm{NaHCO}_{3}$ solution and extracted three times with dichloromethane; the combined organic extracts were dried over $\mathrm{Na}_{2} \mathrm{SO}_{4}$, filtered and evaporated. The residue was purified by flash column chromatography (hexane/ethyl acetate, 2:1) to yield amide $\mathbf{1 b}$ as a white solid $(2.75 \mathrm{~g}, 72 \%)$.

White solid, m. p.: $105-107^{\circ} \mathrm{C} ;{ }^{1} \mathrm{H}$ NMR (400 MHz, $\left.293 \mathrm{~K}, \mathrm{CDCl}_{3}\right): \delta=8.14(\mathrm{~d}, J=4.4 \mathrm{~Hz}, 1 \mathrm{H}), 7.58(\mathrm{~d}, J=7.5 \mathrm{~Hz}, 1 \mathrm{H})$, $7.44(\mathrm{dq}, J=45.9,5.9 \mathrm{~Hz}, 1 \mathrm{H}), 7.02(\mathrm{dd}, J=7.3,5.1 \mathrm{~Hz}, 1 \mathrm{H}), 4.22(\mathrm{t}, J=8.6 \mathrm{~Hz}, 2 \mathrm{H}), 3.18 \mathrm{ppm}(\mathrm{t}, J=8.4 \mathrm{~Hz}, 2 \mathrm{H}) ;{ }^{13} \mathrm{C} \mathrm{NMR}$ $\left(100 \mathrm{MHz}, 293 \mathrm{~K}, \mathrm{CDCl}_{3}\right): \delta=160.3(\mathrm{dq}, J=21.3,2.7 \mathrm{~Hz}), 154.3,146.2,134.5,126.0,121.2(\mathrm{qd}, J=283.0,26.6 \mathrm{~Hz}), 119.6$, $83.0(\mathrm{dq}, J=189.5,33.9 \mathrm{~Hz}), 45.9,24.2 \mathrm{ppm} ;{ }^{19} \mathrm{~F}$ NMR $\left(376 \mathrm{MHz}, 293 \mathrm{~K}, \mathrm{CDCl}_{3}\right): \delta=-75.1$ (s, 3F), -205.7 ppm (m, 1F); IR (film): $\widetilde{v}=3007,2970,2942,2918,1666,1595,1467,1438,1411,1257,1185,1141 \mathrm{~cm}^{-1}$; HRMS (ESI): m/z calculated for $\mathrm{C}_{10} \mathrm{H}_{9} \mathrm{~F}_{4} \mathrm{~N}_{2} \mathrm{O}[\mathrm{M}+\mathrm{H}]^{+}:$249.0646, found: 249.0640 .

In order to determine the conformation of amide rac-1 $\mathbf{b}$ in the solid state, a single-crystal was grown by leaving a concentrated solution of $r a c-1 \mathbf{b}$ in $n$-hexane/ethyl acetate at room temperature. Single-crystal X-ray data were collected on a Rigaku R-AXIS RAPID II imaging plate area detector with graphite-monochromated Cu-K $\alpha$ radiation. Data collection was conducted at $93 \mathrm{~K}$. All structures were solved by direct methods and refined by full matrix least-squares against $F^{2}$ with all reflections. All non-hydrogen atoms were refined anisotropically. All hydrogen atoms were placed in standard calculated positions, and were refined using the riding model. CCDC 1430611 contains the supplementary crystallographic data for rac-1b. Refined structure and crystallographic parameters are summarized in Figure S2 and Table S2. The conformation of amide rac-1 $\mathbf{b}$ in the solid state was determined to be E-conformation. 


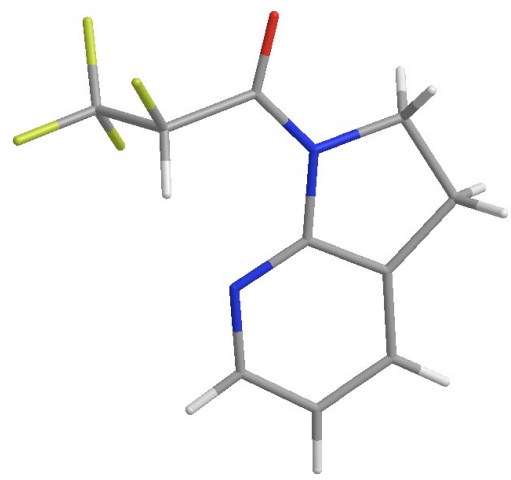

Figure S2. The structure of rac-1b in the solid state (E-conformation). Color code: light green: fluorine; red: oxygen; blue: nitrogen; gray: carbon; white: hydrogen.

\begin{tabular}{c|c}
\hline & rac-1b \\
\hline molecular formula & $\mathrm{C}_{10} \mathrm{H}_{8} \mathrm{~F}_{4} \mathrm{~N}_{2} \mathrm{O}$ \\
formula weight & 248.18 \\
crystal color, habit & colorless, platelet \\
crystal system & monoclinic \\
space group & $C 2 / c$ \\
cell constants: & \\
$a(\AA)$ & $26.8498(13)$ \\
$b(\AA)$ & $4.9574(2)$ \\
$c(\AA)$ & $17.6053(9)$ \\
$\beta(\mathrm{deg})$ & $121.882(9)$ \\
$V\left(\AA{ }^{3}\right)$ & $1989.8(3)$ \\
$\mathrm{Z}$ & 8 \\
$\rho_{\text {calcd. }}\left(\mathrm{g} \cdot \mathrm{cm}^{-3}\right)$ & 1.657 \\
$R_{1}$ & 0.0710 \\
$w R_{2}$ & 0.1860 \\
$F(000)$ & 1008.00 \\
&
\end{tabular}

\section{3,3,4,4,4-Pentafluorobutanoic acid (SI-1).}

$\mathrm{F}_{3} \mathrm{CF}_{2} \mathrm{C} \stackrel{\mathrm{I}}{\mathrm{OH}}_{\mathrm{OH}}$ Jones Reagent (18 mL of a $2.0 \mathrm{M}$ aqueous solution in $\mathrm{H}_{2} \mathrm{SO}_{4}, 36 \mathrm{mmol}, 1.2$ equiv.) was added slowly to a solution of 3,3,4,4,4-pentafluorobutan-1-ol (5.0 g, $30 \mathrm{mmol}, 1.0$ equiv.) in acetone (35 mL, reagent grade) and diethyl ether $\left(15 \mathrm{~mL}\right.$, reagent grade) at $0{ }^{\circ} \mathrm{C}$ under ambient atmosphere. The reaction mixture was stirred for $2 \mathrm{~h}$ at the same temperature before it was carefully quenched by the portionwise addition of solid $\mathrm{NaHSO}_{3}$. The resulting green suspension was diluted with water and extracted three times with diethyl ether; the combined organic extracts were carefully basified using saturated aqueous $\mathrm{NaHCO}_{3}$ solution. After separation of the phases, the organic layer was two times extracted with saturated aqueous $\mathrm{NaHCO}_{3}$ solution. The combined aqueous phase was cooled to $0{ }^{\circ} \mathrm{C}$ and carefully acidified by the dropwise addition of conc. $\mathrm{H}_{2} \mathrm{SO}_{4}$ under vigorous stirring. Then, the solution was extracted three times with diethyl ether; these combined organic extracts were dried over $\mathrm{Na}_{2} \mathrm{SO}_{4}$, filtered and evaporated to afford the title compound SI-1 in pure form as a white crystalline solid ( $3.4 \mathrm{~g}, 62 \%)$.

White crystalline solid, m. p.: $57-59{ }^{\circ} \mathrm{C} ;{ }^{1} \mathrm{H}$ NMR $\left(400 \mathrm{MHz}, 300 \mathrm{~K}, \mathrm{CDCl}_{3}\right): \delta=10.96$ (brs, $\left.1 \mathrm{H}\right), 3.20$ ppm (t, J=16.7 Hz, $2 \mathrm{H}) ;{ }^{13} \mathrm{C} \mathrm{NMR}\left(100 \mathrm{MHz}, 300 \mathrm{~K}, \mathrm{CDCl}_{3}\right): \delta=170.0(\mathrm{t}, J=2.1 \mathrm{~Hz}), 118.5(\mathrm{qt}, J=285.5,35.0 \mathrm{~Hz}), 112.5(\mathrm{tq}, J=256.5,38.9 \mathrm{~Hz})$, $36.4 \mathrm{ppm}(\mathrm{t}, J=22.7 \mathrm{~Hz}) ;{ }^{19} \mathrm{~F}$ NMR $\left(376 \mathrm{MHz}, 300 \mathrm{~K}, \mathrm{CDCl}_{3}\right): \delta=-85.6(\mathrm{~s}, 3 \mathrm{~F}),-116.2 \mathrm{ppm}(\mathrm{t}, J=16.9 \mathrm{~Hz}, 2 \mathrm{~F}) ; \mathrm{IR}(\mathrm{film}): \widetilde{v}=$ 2986 (br), 2959, 1712, 1282, 1211, 1194, 1116, 1034, $918 \mathrm{~cm}^{-1}$; HRMS (ESI): $m / z$ calculated for $\mathrm{C}_{4} \mathrm{H}_{2} \mathrm{~F}_{5} \mathrm{O}_{2} \mathrm{Na}_{2}\left[\mathrm{M}-\mathrm{H}_{+} 2 \mathrm{Na}^{+}\right.$: 222.9765, found: 222.9763 .

\section{1-(2,3-Dihydro-1H-pyrrolo[2,3-b]pyridin-1-yl)-3,3,4,4,4-pentafluorobutan-1-one (1c).}

2,3-Dihydro-7-azaindole (360 mg, $3.0 \mathrm{mmol}, 1.0$ equiv.), triethylamine (0.84 mL, $6.0 \mathrm{mmol}, 2.0$ equiv.) and $\mathrm{T} 3 \mathrm{P}^{\circledR}$ solution $(2.4 \mathrm{~g}$ of a $50 \%$ solution in ethyl acetate, $3.8 \mathrm{mmol}, 1.25$ equiv.) were subsequently added to a solution of 3,3,4,4,4-pentafluorobutanoic acid SI-1 (550 mg, $3.1 \mathrm{mmol}, 1.03$ equiv.) in dichloromethane $(15 \mathrm{~mL})$ at $0{ }^{\circ} \mathrm{C}$. The reaction mixture was slowly warmed to room temperature over $12 \mathrm{~h}$ before it was diluted with saturated aqueous $\mathrm{NaHCO}_{3}$ solution and extracted three times with dichloromethane; the combined organic extracts 

were dried over $\mathrm{Na}_{2} \mathrm{SO}_{4}$, filtered and evaporated. The residue was purified by flash column chromatography (hexane/ethyl acetate, 4:1) to yield amide 1c as a beige fluffy solid (702 $\mathrm{mg}, 84 \%$ ).

Beige fluffy solid, m. p.: $93-94{ }^{\circ} \mathrm{C} ;{ }^{1} \mathrm{H}$ NMR $\left(400 \mathrm{MHz}, 300 \mathrm{~K}, \mathrm{CDCl}_{3}\right): \delta=8.10(\mathrm{~d}, J=4.6 \mathrm{~Hz}, 1 \mathrm{H}), 7.53-7.50(\mathrm{~m}, 1 \mathrm{H}), 6.94$ $(\mathrm{dd}, J=7.4,5.1 \mathrm{~Hz}, 1 \mathrm{H}), 4.27(\mathrm{t}, J=17.8 \mathrm{~Hz}, 2 \mathrm{H}), 4.16(\mathrm{t}, J=8.5 \mathrm{~Hz}, 2 \mathrm{H}), 3.09 \mathrm{ppm}(\mathrm{t}, J=8.5 \mathrm{~Hz}, 2 \mathrm{H}) ;{ }^{13} \mathrm{C} \mathrm{NMR}(100 \mathrm{MHz}$, $\left.300 \mathrm{~K}, \mathrm{CDCl}_{3}\right): \delta=162.0,155.0,145.9,133.9,126.0,118.8(\mathrm{qt}, J=285.8,35.6 \mathrm{~Hz}), 118.7(6), 113.9$ (tq, $\left.J=255.9,38.3 \mathrm{~Hz}\right), 45.8$, $36.6(\mathrm{t}, J=20.1 \mathrm{~Hz}), 23.9 \mathrm{ppm} ;{ }^{19} \mathrm{~F} \mathrm{NMR}\left(376 \mathrm{MHz}, 300 \mathrm{~K}, \mathrm{CDCl}_{3}\right): \delta=-85.6(\mathrm{~s}, 3 \mathrm{~F}),-115.5 \mathrm{ppm}(\mathrm{t}, J=17.7 \mathrm{~Hz}, 2 \mathrm{~F}) ; \mathrm{IR}$ (film): $\widetilde{v}=3001,2952,1669,1600,1591,1431,1346,1193,1098,1022 \mathrm{~cm}^{-1}$; HRMS (ESI): m/z calculated for $\mathrm{C}_{11} \mathrm{H}_{10} \mathrm{~F}_{5} \mathrm{~N}_{2} \mathrm{O}$ $[\mathrm{M}+\mathrm{H}]^{+}:$281.0708, found: 281.0709 .

\section{4-Bromo-1-(2,3-dihydro-1H-pyrrolo[2,3-b]pyridin-1-yl)-3,3,4,4-tetrafluorobutan-1-one (1d).}

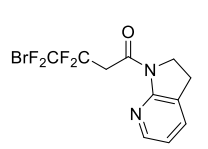

2,3-Dihydro-7-azaindole (360 mg, $3.0 \mathrm{mmol}, 1.0$ equiv.), triethylamine (0.84 mL, $6.0 \mathrm{mmol}, 2.0$ equiv.) and $\mathrm{T} 3 \mathrm{P}^{\circledR}$ solution ( $2.4 \mathrm{~g}$ of a $50 \%$ solution in ethyl acetate, $3.8 \mathrm{mmol}, 1.25$ equiv.) were subsequently added to a solution of 4-bromo-3,3,4,4-tetrafluorobutanoic acid ${ }^{5}$ (741 mg, $3.1 \mathrm{mmol}, 1.03$ equiv.) in dichloromethane $(15 \mathrm{~mL})$ at $0{ }^{\circ} \mathrm{C}$. The reaction mixture was slowly warmed to room temperature over $12 \mathrm{~h}$ before it was diluted with saturated aqueous $\mathrm{NaHCO}_{3}$ solution and extracted three times with dichloromethane; the combined organic extracts were dried over $\mathrm{Na}_{2} \mathrm{SO}_{4}$, filtered and evaporated. The residue was purified by flash column chromatography (hexane/ethyl acetate, 3:1) to yield amide $\mathbf{1 d}$ as a beige fluffy solid (821 $\mathrm{mg}, 75 \%$ ).

Beige fluffy solid, m. p.: $87-89^{\circ} \mathrm{C} ;{ }^{1} \mathrm{H}$ NMR $\left(400 \mathrm{MHz}, 300 \mathrm{~K}, \mathrm{CDCl}_{3}\right): \delta=8.12(\mathrm{~d}, J=5.0 \mathrm{~Hz}, 1 \mathrm{H}), 7.53-7.51(\mathrm{~m}, 1 \mathrm{H}), 6.95$ $(\mathrm{dd}, J=7.4,5.0 \mathrm{~Hz}, 1 \mathrm{H}), 4.34(\mathrm{t}, J=17.8 \mathrm{~Hz}, 2 \mathrm{H}), 4.18(\mathrm{t}, J=8.5 \mathrm{~Hz}, 2 \mathrm{H}), 3.11 \mathrm{ppm}(\mathrm{t}, J=8.5 \mathrm{~Hz}, 2 \mathrm{H}) ;{ }^{13} \mathrm{C} \mathrm{NMR}(100 \mathrm{MHz}$, $\left.300 \mathrm{~K}, \mathrm{CDCl}_{3}\right): \delta=162.3,155.1,146.0,133.9,126.0,118.8,117.4(\mathrm{tt}, J=312.3,39.1 \mathrm{~Hz}), 115.5(\mathrm{tt}, J=258.1,31.7 \mathrm{~Hz}), 45.9$, $36.3(\mathrm{t}, J=20.3 \mathrm{~Hz}), 24.0 \mathrm{ppm} ;{ }^{19} \mathrm{~F}$ NMR $\left(376 \mathrm{MHz}, 300 \mathrm{~K}, \mathrm{CDCl}_{3}\right): \delta=-65.6(\mathrm{~s}, 2 \mathrm{~F}),-109.2 \mathrm{ppm}(\mathrm{t}, J=17.8 \mathrm{~Hz}, 2 \mathrm{~F}) ; \mathrm{IR}$ (film): $\widetilde{v}=2997,2952,1670,1600,1587,1441,1425,1406,1317,1266,1204,1141,1102,1092,877,793$ cm-1; HRMS (ESI): $m / z$ calculated for $\mathrm{C}_{11} \mathrm{H}_{10} \mathrm{BrF}_{4} \mathrm{~N}_{2} \mathrm{O}[\mathrm{M}+\mathrm{H}]^{+}: 340.9907$, found: 340.9910 .

\section{1-(2,3-Dihydro-1H-pyrrolo[2,3-b]pyridin-1-yl)-2-fluoroethan-1-one (1e).}

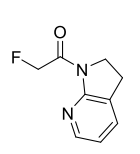

Note that every step of this experiment was performed in a well-ventilated fume hood. Appropriate gloves were used to avoid direct skin contact at any time; special care was also taken during purification as small amounts of highly toxic fluoroacetic acid or its corresponding carboxylates might be present in the reaction mixture!

A flask was charged with lanthanum(III) trifluoromethanesulfonate ( $2.9 \mathrm{~g}, 5.0 \mathrm{mmol}, 0.2 \mathrm{equiv}$.) and flame dried under vacuum for $15 \mathrm{~min}$. After the flask cooled down to ambient temperature, it was backfilled with Ar and subsequently charged with 2,3-dihydro-7-azaindole (3.0 g, $25.0 \mathrm{mmol}, 1.0$ equiv.) and ethyl fluoroacetate (12 mL, $125 \mathrm{mmol}, 5.0$ equiv.). The resulting reaction mixture was stirred for $72 \mathrm{~h}$ at ambient temperature before it was directly loaded onto a silica gel column (hexane/ethyl acetate, 2:1). Amide 1e was obtained as a white solid (2.9 g, 65\%).

White solid, m. p.: $142-144{ }^{\circ} \mathrm{C} ;{ }^{1} \mathrm{H}$ NMR (400 MHz, $\left.293 \mathrm{~K}, \mathrm{CDCl}_{3}\right): \delta=8.08(\mathrm{~d}, J=5.0 \mathrm{~Hz}, 1 \mathrm{H}), 7.50(\mathrm{~d}, J=7.3 \mathrm{~Hz}, 1 \mathrm{H})$, $6.92(\mathrm{dd}, J=7.3,5.0 \mathrm{~Hz}, 1 \mathrm{H}), 5.60(\mathrm{~d}, J=48.5 \mathrm{~Hz}, 2 \mathrm{H}), 4.16(\mathrm{t}, J=8.6 \mathrm{~Hz}, 2 \mathrm{H}), 3.17 \mathrm{ppm}(\mathrm{t}, J=8.5 \mathrm{~Hz}, 2 \mathrm{H}) ;{ }^{13} \mathrm{C} \mathrm{NMR}(100$ $\left.\mathrm{MHz}, 293 \mathrm{~K}, \mathrm{CDCl}_{3}\right): \delta=167.1(\mathrm{~d}, J=20.1 \mathrm{~Hz}), 155.3,146.2,133.7,125.2,118.4,81.4(\mathrm{~d}, J=176.4 \mathrm{~Hz}), 44.7,24.7 \mathrm{ppm}$; ${ }^{19} \mathrm{~F}$ 

$\operatorname{NMR}\left(376 \mathrm{MHz}, 293 \mathrm{~K}, \mathrm{CDCl}_{3}\right): \delta=-226.9 \mathrm{ppm}(\mathrm{m})$; IR (film): $\widetilde{v}=2963,2928,1674,1593,1446,1421,1315,1239,807 \mathrm{~cm}-1$; HRMS (ESI): $m / z$ calculated for $\mathrm{C}_{9} \mathrm{H}_{9} \mathrm{FN}_{2} \mathrm{ONa}[\mathrm{M}+\mathrm{Na}]^{+}: 203.0591$, found: 203.0592.

In order to determine the conformation of amide 1e in the solid state, a single-crystal was grown by leaving a concentrated solution of 1e in $n$-hexane/ethyl acetate at room temperature. Single-crystal X-ray data were collected on a Rigaku R-AXIS RAPID II imaging plate area detector with graphite-monochromated Cu-K $\alpha$ radiation. Data collection was conducted at $93 \mathrm{~K}$. All structures were solved by direct methods and refined by full matrix least-squares against $F^{2}$ with all reflections. All non-hydrogen atoms were refined anisotropically. All hydrogen atoms were placed in standard calculated positions, and were refined using the riding model. CCDC 1430615 contains the supplementary crystallographic data for 1e. Refined structure and crystallographic parameters are summarized in Figure S3 and Table S3. The conformation of amide 1e in the solid state was determined to be E-conformation.

Table S3. Selected Crystallographic Data of 1e

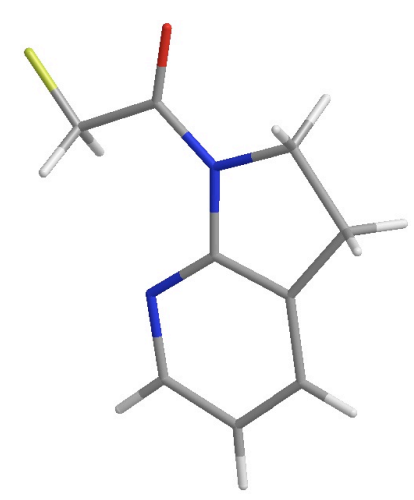

Figure S3. The structure of 1e in the solid state (E-conformation). Color code: light green: fluorine; red: oxygen; blue: nitrogen; gray: carbon; white: hydrogen.

\begin{tabular}{c|c}
\hline & $\mathbf{1 e}$ \\
\hline molecular formula & $\mathrm{C}_{18} \mathrm{H}_{18} \mathrm{~F}_{2} \mathrm{~N}_{4} \mathrm{O}_{2}$ \\
formula weight & 360.36 \\
crystal color, habit & colorless, prism \\
crystal system & monoclinic \\
space group & $P 21 / n$ \\
cell constants: & \\
$a(\AA)$ & $7.0914(3)$ \\
$b(\AA)$ & $26.3930(6)$ \\
$c(\AA)$ & $8.8755(3)$ \\
$\beta(\mathrm{deg})$ & $107.452(8)$ \\
$V\left(\AA^{3}\right)$ & $1584.70(11)$ \\
$Z$ & 4 \\
$\rho_{\text {calcd. }}\left(\mathrm{g} \cdot \mathrm{cm}^{-3}\right)$ & 1.510 \\
$R_{1}$ & 0.0489 \\
$w R_{2}$ & 0.1018 \\
$F(000)$ & 752.00
\end{tabular}

\section{1-(2,3-Dihydro-1H-pyrrolo[2,3-b]pyridin-1-yl)-3,4,4,4-tetrafluoro-3-(trifluoromethyl)butan-1-one (1f).}

Following the above described $\mathrm{T}^{3} \mathrm{P}^{\circledR}$-mediated coupling reaction for the preparation of amide $\mathbf{1 d}$, amide $\mathbf{1 f}$ was obtained from 2,3-dihydro-7-azaindole and 3,4,4,4-tetrafluoro-3-(trifluoromethly)butanoic acid. The crude residue was purified by flash column chromatography (hexane/ethyl acetate, 4:1) to yield amide 1f as a white solid. However, the target material is instable and some byproduct resulting from decomposition is always present (directly after flash chromatography: $<15 \%$ based on ${ }^{1} \mathrm{H}$ analysis). Below given are ${ }^{1} \mathrm{H}$ and ${ }^{19} \mathrm{~F}$ NMR data as well as HRMS of the desired major component:

${ }^{1} \mathrm{H}$ NMR $\left(400 \mathrm{MHz}, 300 \mathrm{~K}, \mathrm{CDCl}_{3}\right): \delta=8.12(\mathrm{~d}, J=5.0 \mathrm{~Hz}, 1 \mathrm{H}), 7.53(\mathrm{dq}, J=7.4,1.3 \mathrm{~Hz}, 1 \mathrm{H}), 6.95(\mathrm{dd}, J=7.4,5.1 \mathrm{~Hz}, 1 \mathrm{H})$, $4.40(\mathrm{~d}, J=20.9 \mathrm{~Hz}, 2 \mathrm{H}), 4.16(\mathrm{t}, J=8.5 \mathrm{~Hz}, 2 \mathrm{H}), 3.10 \mathrm{ppm}(\mathrm{t}, J=8.5 \mathrm{~Hz}, 2 \mathrm{H}) ;{ }^{19} \mathrm{~F}$ NMR $(376 \mathrm{MHz}, 300 \mathrm{~K}, \mathrm{CDCl}): \delta=-76.3$ (s, 6F), -182.3 ppm (s, 1F); HRMS (ESI): m/z calculated for $\mathrm{C}_{12} \mathrm{H}_{9} \mathrm{~F}_{7} \mathrm{~N}_{2} \mathrm{ONa}[\mathrm{M}+\mathrm{Na}]^{+}: 353.0495$, found: 353.0490 . 


\section{3,3,4,4,5,5,5-Heptafluoropentanoic acid (SI-2).}

$\mathrm{F}_{3} \mathrm{CF}_{2} \mathrm{CF}_{2} \mathrm{C}{ }_{\mathrm{O}_{\mathrm{OH}}}$ Jones Reagent (10 mL of a $2.0 \mathrm{M}$ aqueous solution in $\mathrm{H}_{2} \mathrm{SO}_{4}, 20 \mathrm{mmol}, 2.0$ equiv.) was added slowly to a solution of 3,3,4,4,5,5,5-heptafluoropentan-1-ol (2.1 g, $10 \mathrm{mmol}, 1.0$ equiv.) in acetone (10 $\mathrm{mL}$, reagent grade) and diethyl ether $\left(5 \mathrm{~mL}\right.$, reagent grade) at $0{ }^{\circ} \mathrm{C}$ under ambient atmosphere. The reaction mixture was stirred for $2 \mathrm{~h}$ at the same temperature before it was carefully quenched by the portionwise addition of solid $\mathrm{NaHSO}_{3}$. The resulting green suspension was diluted with water and extracted three times with diethyl ether; the combined organic extracts were carefully basified using saturated aqueous $\mathrm{NaHCO}_{3}$ solution. After separation of the phases, the organic layer was two times extracted with saturated aqueous $\mathrm{NaHCO}_{3}$ solution. The combined aqueous phase was cooled to $0{ }^{\circ} \mathrm{C}$ and carefully acidified by the dropwise addition of conc. $\mathrm{H}_{2} \mathrm{SO}_{4}$ under vigorous stirring. Then, the solution was extracted three times with diethyl ether; these combined organic extracts were dried over $\mathrm{Na}_{2} \mathrm{SO}_{4}$, filtered and evaporated to afford the title compound SI-2 as a clear slightly yellow oil (1.34 $\mathrm{g}, 59 \%)$.

${ }^{1} \mathrm{H}$ NMR (400 MHz, $\left.300 \mathrm{~K}, \mathrm{CDCl}_{3}\right): \delta=10.85$ (brs, 1H), 3.22 ppm (t, $\left.J=17.2 \mathrm{~Hz}, 2 \mathrm{H}\right) ;{ }^{13} \mathrm{C} \mathrm{NMR}\left(100 \mathrm{MHz}, 300 \mathrm{~K}, \mathrm{CDCl}_{3}\right)$ : $\delta=170.3,117.6(\mathrm{qt}, J=286.6,33.7 \mathrm{~Hz}), 114.7(\mathrm{tt}, J=257.8,31.8 \mathrm{~Hz}), 108.4(\mathrm{tqt}, J=264.6,38.4,34.8 \mathrm{~Hz}), 36.4 \mathrm{ppm}(\mathrm{t}, J=22.7$ $\mathrm{Hz}$ ); ${ }^{19} \mathrm{~F}$ NMR (376 MHz, $\left.300 \mathrm{~K}, \mathrm{CDCl}_{3}\right): \delta=-80.4$ (s, 3F), -113.0 (s, 2F), -127.2 ppm (s, 2F); IR (film): $\widetilde{v}=3032$ (br), 1739 , 1428, 1356, 1226, 1177, 1118, 968, $748 \mathrm{~cm}^{-1}$; HRMS (ESI): $\mathrm{m} / z$ calculated for $\mathrm{C}_{5} \mathrm{H}_{2} \mathrm{~F}_{7} \mathrm{O}_{2} \mathrm{Na} 2[\mathrm{M}-\mathrm{H}+2 \mathrm{Na}]^{+}: 272.9733$, found: 272.9730.

\section{1-(2,3-Dihydro-1H-pyrrolo[2,3-b]pyridin-1-yl)-3,3,4,4,5,5,5-heptafluoropentan-1-one (1g).}

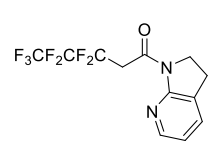

2,3-Dihydro-7-azaindole ( $240 \mathrm{mg}, 2.0 \mathrm{mmol}, 1.0$ equiv.), triethylamine (0.56 mL, $4.0 \mathrm{mmol}, 2.0$ equiv.) and $\mathrm{T} 3 \mathrm{P}^{\circledR}$ solution ( $1.6 \mathrm{~g}$ of a $50 \%$ solution in ethyl acetate, $1.9 \mathrm{mmol}, 1.25$ equiv.) were subsequently added to a solution of 3,3,4,4,5,5,5-heptafluoropentanoic acid SI-2 (479 mg, $2.1 \mathrm{mmol}, 1.05$ equiv.) in dichloromethane $(10 \mathrm{~mL})$ at $0{ }^{\circ} \mathrm{C}$. The reaction mixture was stirred at the same temperature for $3 \mathrm{~h}$ before it was diluted with saturated aqueous $\mathrm{NaHCO}_{3}$ solution and extracted three times with dichloromethane; the combined organic extracts were dried over $\mathrm{Na}_{2} \mathrm{SO}_{4}$, filtered and evaporated. The residue was purified by flash column chromatography (hexane/ethyl acetate, 5:1) to yield amide $1 \mathrm{~g}$ as an orange solid (451 $\mathrm{mg}, 68 \%$ ).

Orange solid, m. p.: $56-58{ }^{\circ} \mathrm{C}$; ${ }^{1} \mathrm{H}$ NMR $\left(400 \mathrm{MHz}, 300 \mathrm{~K}, \mathrm{CDCl}_{3}\right): \delta=8.12(\mathrm{~d}, J=4.8 \mathrm{~Hz}, 1 \mathrm{H}), 7.54-7.51(\mathrm{~m}, 1 \mathrm{H}), 6.95(\mathrm{dd}$, $J=7.4,5.1 \mathrm{~Hz}, 1 \mathrm{H}), 4.33(\mathrm{t}, J=18.3 \mathrm{~Hz}, 2 \mathrm{H}), 4.18(\mathrm{t}, J=8.5 \mathrm{~Hz}, 2 \mathrm{H}), 3.11 \mathrm{ppm}(\mathrm{t}, J=8.5 \mathrm{~Hz}, 2 \mathrm{H}) ;{ }^{13} \mathrm{C} \mathrm{NMR}(125 \mathrm{MHz}$, $\left.298 \mathrm{~K}, \mathrm{CDCl}_{3}\right): \delta=162.0,155.0,146.0,133.9,126.0,118.8,117.8$ (qt, $\left.J=287.5,33.9 \mathrm{~Hz}\right), 116.0(\mathrm{tt}, J=257.3,31.2 \mathrm{~Hz}), 108.6$ $(\mathrm{tqt}, J=264.6,37.9,35.2 \mathrm{~Hz}), 45.9,36.6(\mathrm{t}, J=20.1 \mathrm{~Hz}), 24.0 \mathrm{ppm} ;{ }^{19} \mathrm{~F}$ NMR $(376 \mathrm{MHz}, 300 \mathrm{~K}, \mathrm{CDCl}): \delta=-80.4(\mathrm{~s}, 3 \mathrm{~F})$, -112.3 (s, 2F), -127.5 ppm (s, 2F); IR (film): $\widetilde{v}=3059,3007,2966,1669,1596,1480,1428,1353,1326,1268,1221,1114,972$, $786 \mathrm{~cm}^{-1}$; HRMS (ESI): $m / z$ calculated for $\mathrm{C}_{12} \mathrm{H}_{10} \mathrm{~F}_{7} \mathrm{~N}_{2} \mathrm{O}[\mathrm{M}+\mathrm{H}]^{+}: 331.0676$, found: 331.0675 .

\section{1-(2,3-Dihydro-1H-pyrrolo[2,3-b]pyridin-1-yl)-3,3,3-trifluoro-2-(trifluoromethyl)propan-1-one (1h).}

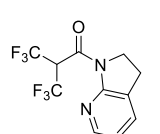

2,3-Dihydro-7-azaindole (240 mg, $2.0 \mathrm{mmol}, 1.0$ equiv.), triethylamine (0.56 mL, $4.0 \mathrm{mmol}, 2.0$ equiv.) and T3P ${ }^{\circledR}$ solution ( $1.6 \mathrm{~g}$ of a $50 \%$ solution in ethyl acetate, $2.5 \mathrm{mmol}, 1.25$ equiv.) were subsequently added to a solution of 3,3,3-trifluoro-2-(trifluoromethly)propanoic acid (471 mg, $2.4 \mathrm{mmol}, 1.2$ equiv.) in 

dichloromethane $(10 \mathrm{~mL})$ at $0{ }^{\circ} \mathrm{C}$. The reaction mixture was slowly warmed to room temperature over $12 \mathrm{~h}$ before it was diluted with saturated aqueous $\mathrm{NaHCO}_{3}$ solution and extracted three times with dichloromethane; the combined organic extracts were dried over $\mathrm{Na}_{2} \mathrm{SO}_{4}$, filtered and evaporated. The residue was purified by flash column chromatography (hexane/ethyl acetate, 3:1) to yield amide $\mathbf{1 h}$ as a white crystalline solid (428 $\mathrm{mg}, 72 \%)$.

White solid, m. p.: $170-172{ }^{\circ} \mathrm{C} ;{ }^{1} \mathrm{H}$ NMR $\left(400 \mathrm{MHz}, 300 \mathrm{~K}, \mathrm{CDCl}_{3}\right): \delta=8.17-8.15(\mathrm{~m}, 1 \mathrm{H}), 7.60(\mathrm{dq}, J=7.4,1.3 \mathrm{~Hz}, 1 \mathrm{H})$, 7.27 (hept., $J=7.8 \mathrm{~Hz}, 1 \mathrm{H}), 7.04$ (dd, $J=7.4,5.1 \mathrm{~Hz}, 1 \mathrm{H}), 4.26-4.22$ (m, 2H), 3.17-3.13 ppm (m, $2 \mathrm{H}) ;{ }^{13} \mathrm{C} \mathrm{NMR}(125 \mathrm{MHz}$, $300 \mathrm{~K}, \mathrm{CDCl}_{3}$ ): $\delta=157.4$ (hept., $J=2.4 \mathrm{~Hz}$ ), 154.2, 145.9, 134.6, 126.4, 121.7 (m), 119.6, 50.6 (hept., J = 29.0 Hz), $46.3,23.9$ ppm; ${ }^{19} \mathrm{~F}$ NMR (376 MHz, $\left.300 \mathrm{~K}, \mathrm{CDCl}_{3}\right): \delta=-63.6$ ppm (s); IR (film): $\widetilde{v}=3018,2922,1665,1597,1448,1432,1415,1380$, 1295, 1275, 1239, 1217, 1173, 1158, 1094, 920, 868, $770 \mathrm{~cm}^{-1}$; HRMS (ESI): $m / z$ calculated for $\mathrm{C}_{11} \mathrm{H}_{8} \mathrm{~F}_{6} \mathrm{~N}_{2} \mathrm{ONa}[\mathrm{M}+\mathrm{Na}]^{+}$: 321.0433, found: 321.0431 .

\section{1-(2,3-Dihydro-1H-pyrrolo[2,3-b]pyridin-1-yl)-3,3,3-trifluoro-2-methylpropan-1-one (1i).}

Following the above described $\mathrm{T} 3 \mathrm{P}^{\circledR}$-mediated coupling reaction for the preparation of amide $\mathbf{1 h}$, amide $\mathbf{1 i}$ was obtained from 3,3,3-trifluoro-2-methylpropanoic acid and 2,3-dihydro-7-azaindole.

White solid, m. p.: $95-97^{\circ} \mathrm{C} ;{ }^{1} \mathrm{H}$ NMR $\left(400 \mathrm{MHz}, 300 \mathrm{~K}, \mathrm{CDCl}_{3}\right): \delta=8.13(\mathrm{~d}, J=5.1 \mathrm{~Hz}, 1 \mathrm{H}), 7.53-7.50(\mathrm{~m}, 1 \mathrm{H}), 6.94(\mathrm{dd}, J$ $=7.4,5.1 \mathrm{~Hz}, 1 \mathrm{H}), 5.59-5.48(\mathrm{~m}, 1 \mathrm{H}), 4.24-4.16(\mathrm{~m}, 1 \mathrm{H}), 4.14-4.06(\mathrm{~m}, 1 \mathrm{H}), 3.16-3.02(\mathrm{~m}, 2 \mathrm{H}), 1.47 \mathrm{ppm}(\mathrm{m}, 3 \mathrm{H}) ;{ }^{13} \mathrm{C} \mathrm{NMR}$ $\left(100 \mathrm{MHz}, 300 \mathrm{~K}, \mathrm{CDCl}_{3}\right): \delta=167.3(\mathrm{q}, J=3.0 \mathrm{~Hz}), 155.1,146.1,133.9,126.2,125.9(\mathrm{q}, J=279.5 \mathrm{~Hz}), 118.7,45.9,42.3(\mathrm{q}, J=$ 27.1 Hz), 23.9, 11.4 ppm (q, $J=2.7 \mathrm{~Hz}) ;{ }^{19} \mathrm{~F}$ NMR $\left(376 \mathrm{MHz}, 300 \mathrm{~K}, \mathrm{CDCl}_{3}\right): \delta=-68.9 \mathrm{ppm}(\mathrm{s}) ; \mathrm{IR}($ film $): \tilde{v}=2973,2946$, 1655, 1602, 1445, 1417, 1297, 1265, 1238, 1169, 1129, 1010, $802 \mathrm{~cm}^{-1}$; HRMS (ESI): m/z calculated for $\mathrm{C}_{11} \mathrm{H}_{12} \mathrm{~F}_{3} \mathrm{~N}_{2} \mathrm{O}[\mathrm{M}+\mathrm{H}]^{+}$: 245.0896, found: 245.0891 .

\section{3,3,3-Trifluoro-1-(1H-pyrrolo[2,3-b]pyridin-1-yl)propan-1-one (1aa).}

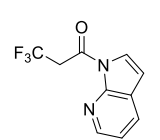

3,3,3-Trifluoropropionyl chloride (1.0 $\mathrm{mL}, 9.7 \mathrm{mmol}, 1.1$ equiv.) was added dropwise to a suspension of 7-azaindole (1.04 g, $8.8 \mathrm{mmol}, 1.0$ equiv.), $\mathrm{K}_{2} \mathrm{CO}_{3}\left(2.43 \mathrm{~g}\right.$, $17.6 \mathrm{mmol}, 2.0$ equiv.), and THF $(30 \mathrm{~mL})$ at $0{ }^{\circ} \mathrm{C}$. The suspension was stirred for $6 \mathrm{~h}$ at room temperature before it was diluted with water and extracted three times with ethyl acetate; the combined organic extracts were dried over $\mathrm{Na}_{2} \mathrm{SO}_{4}$, filtered and evaporated. The residue was purified by flash column chromatography (hexane/ethyl acetate, 6:1) to yield amide 1aa as a white fluffy solid (815 mg, 41\%).

White fluffy solid, m. p.: $86-88^{\circ} \mathrm{C} ;{ }^{1} \mathrm{H}$ NMR $\left(400 \mathrm{MHz}, 293 \mathrm{~K}, \mathrm{CDCl}_{3}\right): \delta=8.35$ (dd, J = 4.8, $\left.1.4 \mathrm{~Hz}, 1 \mathrm{H}\right), 7.97(\mathrm{~d}, J=4.1 \mathrm{~Hz}$, $1 \mathrm{H}), 7.90(\mathrm{dd}, J=7.8,1.4 \mathrm{~Hz}, 1 \mathrm{H}), 7.24(\mathrm{dd}, J=7.8,4.8 \mathrm{~Hz}, 1 \mathrm{H}), 6.66(\mathrm{~d}, J=4.1 \mathrm{~Hz}, 1 \mathrm{H}), 4.62 \mathrm{ppm}(\mathrm{q}, J=10.0 \mathrm{~Hz}, 2 \mathrm{H}) ;{ }^{13} \mathrm{C}$ $\operatorname{NMR}\left(100 \mathrm{MHz}, 293 \mathrm{~K}, \mathrm{CDCl}_{3}\right): \delta=162.1$ (q, J = 4.2 Hz), 147.4, 143.9, 129.8, 125.0, 123.8(2), 123.8 (q, J = 276.4 Hz), 119.4, 107.1, 41.7 ppm (q, J = 29.4 Hz); ${ }^{19} \mathrm{~F}$ NMR (376 MHz, $\left.293 \mathrm{~K}, \mathrm{CDCl}_{3}\right): \delta=-62.3 \mathrm{ppm}(\mathrm{m}) ; \mathrm{IR}(\mathrm{film}): \widetilde{v}=3156,2994,2949$, 1715, 1534, 1421, 1406, 1387, 1320, 1269, 1254, 1220, 1197, 1136, 1116, $1102 \mathrm{~cm}^{-1}$; HRMS (ESI): m/z calculated for $\mathrm{C}_{10} \mathrm{H}_{7} \mathrm{~F}_{3} \mathrm{~N}_{2} \mathrm{ONa}[\mathrm{M}+\mathrm{Na}]^{+}: 251.0403$, found: 251.0399 . 


\section{1-(2,3-Dihydro-1H-pyrrolo[3,2-c]pyridin-1-yl)-3,3,3-trifluoropropan-1-one (1ab).}

等

Following the above described coupling reaction for the preparation of amide 1aa, amide 1ab was obtained from 3,3,3-trifluoropropionyl chloride and 2,3-dihydro-5-azaindole and purified by flash column chromatography (ethyl acetate/hexane, 3:1 and 10\% triethylamine).

White solid, m. p.: $131-133{ }^{\circ} \mathrm{C}$; ${ }^{1} \mathrm{H}$ NMR (400 MHz, $373 \mathrm{~K}$, DMSO- $\left.d_{6}\right): \delta=8.40(\mathrm{~s}, 1 \mathrm{H}), 8.34(\mathrm{~d}, J=5.3 \mathrm{~Hz}, 1 \mathrm{H}), 7.83(\mathrm{~d}, J=$ $5.0 \mathrm{~Hz}, 1 \mathrm{H}), 4.18(\mathrm{t}, J=8.5 \mathrm{~Hz}, 2 \mathrm{H}), 3.78(\mathrm{q}, J=10.6 \mathrm{~Hz}, 2 \mathrm{H}), 3.21 \mathrm{ppm}(\mathrm{t}, J=8.6 \mathrm{~Hz}, 2 \mathrm{H}) ;{ }^{13} \mathrm{C} \mathrm{NMR}(100 \mathrm{MHz}, 373 \mathrm{~K}$, DMSO-d $d): \delta=162.7(\mathrm{~m}), 148.4,148.3,145.6,127.2,121.3(\mathrm{q}, J=276.0 \mathrm{~Hz}), 110.1,47.5,39.0(\mathrm{q}, J=28.1 \mathrm{~Hz}), 24.7 \mathrm{ppm}$; ${ }^{19} \mathrm{~F}$ NMR (376 MHz, $\left.373 \mathrm{~K}, \mathrm{DMSO}-d_{6}\right): \delta=-61.1 \mathrm{ppm}(\mathrm{t}, J=11.6 \mathrm{~Hz})$; IR (film): $\widetilde{v}=3357,3121,2973,2933,1683,1595,1492$, 1480, 1441, 1424, 1406, 1376, 1338, 1288, 1259, 1227, 1173, 1111, $853 \mathrm{~cm}^{-1}$; HRMS (ESI): $\mathrm{m} / z$ calculated for $\mathrm{C}_{10} \mathrm{H}_{10} \mathrm{~F}_{3} \mathrm{~N}_{2} \mathrm{O}$ $[\mathrm{M}+\mathrm{H}]^{+}: 231.0740$, found: 231.0735 .

\section{3,3,3-Trifluoro-1-(indolin-1-yl)propan-1-one (1ac).}

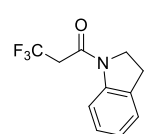

Following the above described coupling reaction for the preparation of amide 1aa, amide 1ac was obtained from 3,3,3-trifluoropropionyl chloride and 2,3-dihydroindole and purified by flash column chromatography (hexane/ethyl acetate, 6:1).

White crystalline solid, m.p.: $136-137{ }^{\circ} \mathrm{C} ;{ }^{1} \mathrm{H}$ NMR (400 MHz, $343 \mathrm{~K}$, DMSO-d6): $\delta=8.05$ (brs, $\left.1 \mathrm{H}\right), 7.24(\mathrm{~d}, J=7.3 \mathrm{~Hz}$, $1 \mathrm{H}), 7.16(\mathrm{t}, J=7.4 \mathrm{~Hz}, 1 \mathrm{H}), 7.02(\mathrm{td}, J=7.4,0.9 \mathrm{~Hz}, 1 \mathrm{H}), 4.12(\mathrm{t}, J=8.5 \mathrm{~Hz}, 2 \mathrm{H}), 3.73(\mathrm{q}, J=10.8 \mathrm{~Hz}, 2 \mathrm{H}), 3.15 \mathrm{ppm}(\mathrm{t}, J=$ $8.2 \mathrm{~Hz}, 2 \mathrm{H}) ;{ }^{13} \mathrm{C}$ NMR $\left(100 \mathrm{MHz}, 343 \mathrm{~K}, \mathrm{DMSO}-d_{6}\right): \delta=161.3(\mathrm{q}, J=3.2 \mathrm{~Hz}), 141.9,131.5,126.5,124.4,124.2(\mathrm{q}, J=275.7$ $\mathrm{Hz}), 123.3,115.7,47.2,38.8(\mathrm{q}, J=27.8 \mathrm{~Hz}), 26.8 \mathrm{ppm} ;{ }^{19} \mathrm{~F}$ NMR (376 MHz, $\left.343 \mathrm{~K}, \mathrm{DMSO}-d_{6}\right): \delta=-61.0 \mathrm{ppm}(\mathrm{t}, J=11.6$ Hz); IR (film): $\widetilde{v}=2966,2946,1672,1483,1432,1404,1370,1282,1263,1173,1132,1105 \mathrm{~cm}^{-1}$; HRMS (ESI): $\mathrm{m} / z$ calculated for $\mathrm{C}_{11} \mathrm{H}_{10} \mathrm{~F}_{3} \mathrm{NONa}[\mathrm{M}+\mathrm{Na}]^{+}: 252.0607$, found: 252.0599 .

\section{3,3,3-Trifluoro- $N$-methyl- $N$-(pyridin-2-yl)propanamide (1ad).}

$\mathrm{F}_{3} \mathrm{C}$. 3,3,3-Trifluoropropionyl chloride $(1.0 \mathrm{~mL}, 9.7 \mathrm{mmol}, 1.0$ equiv.) was added dropwise to a solution of 2-(methylamino)pyridine ( $1.1 \mathrm{~g}, 10 \mathrm{mmol}, 1.03$ equiv.) and triethylamine ( $2.8 \mathrm{~mL}, 20 \mathrm{mmol}, 2.1$ equiv.) in dichloromethane $(50 \mathrm{~mL})$. The reaction mixture was stirred for $2 \mathrm{~h}$ before it was diluted with water and extracted three times with dichloromethane; the combined organic extracts were dried over $\mathrm{Na}_{2} \mathrm{SO}_{4}$, filtered and evaporated. The residue was purified by flash column chromatography (hexane/ethyl acetate, 4:3) to yield amide 1ad as a white solid $(1.6 \mathrm{~g}, 72 \%)$.

White solid, m.p.: $31{ }^{\circ} \mathrm{C}$; ${ }^{1} \mathrm{H}$ NMR $\left(400 \mathrm{MHz}, 293 \mathrm{~K}, \mathrm{CDCl}_{3}\right): \delta=8.47(\mathrm{~d}, J=3.9 \mathrm{~Hz}, 1 \mathrm{H}), 7.80(\mathrm{dd}, J=7.7,7.5 \mathrm{~Hz}, 1 \mathrm{H})$, 7.26-7.21 (m, 2H), $3.36(\mathrm{~s}, 3 \mathrm{H}), 3.28 \mathrm{ppm}(\mathrm{q}, J=10.1 \mathrm{~Hz}, 2 \mathrm{H}) ;{ }^{13} \mathrm{C}$ NMR $\left(100 \mathrm{MHz}, 293 \mathrm{~K}, \mathrm{CDCl}_{3}\right): \delta=163.6(\mathrm{q}, J=3.5 \mathrm{~Hz})$, 155.2, 149.0, 138.9, 124.0 (q, J = 276.7 Hz), 122.5, 119.8, 39.2 (q, J = 28.8 Hz), 35.7 ppm; ${ }^{19} \mathrm{~F}$ NMR (376 MHz, $\left.293 \mathrm{~K}, \mathrm{CDCl}_{3}\right):$ $\delta=-62.4 \mathrm{ppm}(\mathrm{t}, J=11.6 \mathrm{~Hz}) ; \mathrm{IR}\left(\right.$ film): $\widetilde{v}=3062,2949,1678,1588,1573,1474,1437,1394,1340,1249,1114,852 \mathrm{~cm}^{-1}$; HRMS (ESI): $m / z$ calculated for $\mathrm{C}_{9} \mathrm{H}_{9} \mathrm{~F}_{3} \mathrm{~N}_{2} \mathrm{ONa}[\mathrm{M}+\mathrm{Na}]^{+}: 241.0559$, found: 241.0554 . 


\section{3,3,3-Trifluoro- $N$-(pyridin-2-yl)propanamide (1ae).}

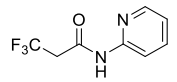

3,3,3-Trifluoropropionyl chloride (1.0 $\mathrm{mL}, 9.7 \mathrm{mmol}, 1.0$ equiv.) was added dropwise to a suspension of 2-aminopyridine ( $1.1 \mathrm{~g}, 10 \mathrm{mmol}, 1.03$ equiv.), $\mathrm{NaHCO}_{3}(2.5 \mathrm{~g}, 30 \mathrm{mmol}, 3.0$ equiv.) and dichloromethane $(40 \mathrm{~mL})$. The reaction mixture was stirred for $12 \mathrm{~h}$ before it was diluted with water and extracted three times with dichloromethane; the combined organic extracts were dried over $\mathrm{Na}_{2} \mathrm{SO}_{4}$, filtered and evaporated. The residue was purified by flash column chromatography (hexane/ethyl acetate, 3:1) to yield amide 1ae as a white solid (1.24 g, 61\%). White solid, m. p.: $108{ }^{\circ} \mathrm{C} ;{ }^{1} \mathrm{H}$ NMR $\left(400 \mathrm{MHz}, 293 \mathrm{~K}, \mathrm{CDCl}_{3}\right): \delta=9.87$ (brs, $\left.1 \mathrm{H}\right), 8.28(\mathrm{~d}, J=3.9 \mathrm{~Hz}, 1 \mathrm{H}), 8.22(\mathrm{~d}, J=8.5$ $\mathrm{Hz}, 1 \mathrm{H}), 7.77(\mathrm{ddd}, J=8.7,7.1,1.4 \mathrm{~Hz}, 1 \mathrm{H}), 7.13(\mathrm{ddd}, J=7.4,5.0,1.1 \mathrm{~Hz}, 1 \mathrm{H}), 3.27 \mathrm{ppm}(\mathrm{q}, J=10.2 \mathrm{~Hz}, 2 \mathrm{H}) ;{ }^{13} \mathrm{C}$ NMR $\left(100 \mathrm{MHz}, 293 \mathrm{~K}, \mathrm{CDCl}_{3}\right): \delta=161.6(\mathrm{q}, J=3.5 \mathrm{~Hz}), 151.1,147.4,139.0,123.8(\mathrm{q}, J=277.0 \mathrm{~Hz}), 120.7,115.2,42.2 \mathrm{ppm}(\mathrm{q}, J=$ 29.4 Hz); ${ }^{19} \mathrm{~F}$ NMR (376 MHz, $\left.293 \mathrm{~K}, \mathrm{CDCl}_{3}\right): \delta=-62.6 \mathrm{ppm}(\mathrm{t}, J=11.6 \mathrm{~Hz}) ; \mathrm{IR}($ film): $\widetilde{v}=3420$ (br), 3001, 2952, 1672, 1581, 1556, 1439, 1353, 1280, 1259, 1230, 1145, 1081, $945 \mathrm{~cm}^{-1}$; HRMS (ESI): $\mathrm{m} / z$ calculated for $\mathrm{C}_{8} \mathrm{H}_{8} \mathrm{~F}_{3} \mathrm{~N}_{2} \mathrm{O}[\mathrm{M}+\mathrm{H}]^{+}: 205.0583$, found: 205.0581 .

\section{1-((2,2,2-Trifluoroethyl)sulfonyl)-2,3-dihydro-1H-pyrrolo[2,3-b]pyridine (1af).}

F 3 C,2,2-Trifluoroethane-1-sulfonyl chloride $(0.37 \mathrm{~mL}, 3.3 \mathrm{mmol}, 1.1$ equiv.) was added dropwise to a solution of 2,3-dihydro-7-azaindole ( $360.5 \mathrm{mg}, 3.0 \mathrm{mmol}, 1.0$ equiv.) and diisopropylethylamine (1.05 mL, $6.0 \mathrm{mmol}$, 2.0 equiv.) in dichloromethane $(9 \mathrm{~mL})$ at $0{ }^{\circ} \mathrm{C}$. The reaction mixture was stirred for $1 \mathrm{~h}$ at the same temperature and $3 \mathrm{~h}$ at room temperature before it was diluted with saturated aqueous $\mathrm{NaHCO}_{3}$ solution and extracted three times with dichloromethane; the combined organic extracts were dried over $\mathrm{Na}_{2} \mathrm{SO}_{4}$, filtered and evaporated. The residue was purified by flash column chromatography (hexane/ethyl acetate, 3:1) to yield sulfonamide 1af as a beige solid (360 mg, $45 \%)$.

Beige solid, m. p.: $106-108{ }^{\circ} \mathrm{C} ;{ }^{1} \mathrm{H}$ NMR $\left(400 \mathrm{MHz}, 300 \mathrm{~K}, \mathrm{CDCl}_{3}\right): \delta=8.17-8.15(\mathrm{~m}, 1 \mathrm{H}), 7.53-7.50(\mathrm{~m}, 1 \mathrm{H}), 6.95(\mathrm{dd}, J=$ 7.4, $5.2 \mathrm{~Hz}, 1 \mathrm{H}), 4.44(\mathrm{q}, J=9.1 \mathrm{~Hz}, 2 \mathrm{H}), 4.18(\mathrm{t}, J=8.5 \mathrm{~Hz}, 2 \mathrm{H}), 3.19 \mathrm{ppm}(\mathrm{t}, J=8.5 \mathrm{~Hz}, 2 \mathrm{H}) ;{ }^{13} \mathrm{C} \mathrm{NMR}(100 \mathrm{MHz}, 300 \mathrm{~K}$, $\left.\mathrm{CDCl}_{3}\right): \delta=155.4,146.8,133.8,124.1,121.5(\mathrm{q}, J=277.4 \mathrm{~Hz}), 118.5,53.1(\mathrm{q}, J=32.0 \mathrm{~Hz}), 47.8,25.4 \mathrm{ppm} ;{ }^{19} \mathrm{~F}$ NMR $(376$ $\left.\mathrm{MHz}, 300 \mathrm{~K}, \mathrm{CDCl}_{3}\right): \delta=-62.4$ ppm (s); IR (film): $\widetilde{v}=3069,2996,2942,1599,1484,1461,1420,1371,1323,1253,1230,1173$, 1137, 1119, 1085, 1038, 993, $791 \mathrm{~cm}^{-1}$; HRMS (ESI): m/z calculated for $\mathrm{C}_{9} \mathrm{H}_{10} \mathrm{~F}_{3} \mathrm{~N}_{2} \mathrm{O}_{2} \mathrm{~S}$ [M+H] $]^{+}$267.0410, found: 267.0410.

\section{3,3,3-Trifluoro- $N, N$-dimethylpropanamide (1ag).}

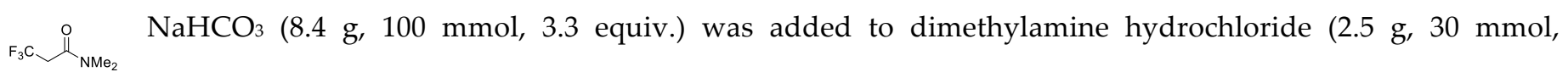
1.0 equiv.) in dichloromethane (100 mL). To the resulting suspension was added 3,3,3-trifluoropropionyl chloride ( $3.1 \mathrm{~mL}, 30 \mathrm{mmol}, 1.0$ equiv.). The reaction mixture was stirred for $4 \mathrm{~h}$ before it was diluted with water and extracted three times with dichloromethane; the combined organic extracts were dried over $\mathrm{Na}_{2} \mathrm{SO}_{4}$, filtered and evaporated. The residue was recrystallized from ethyl acetate/hexane to yield amide 1ag as a white crystalline solid $(4.0 \mathrm{~g}, 86 \%)$. All analytical data match those reported in the literature. ${ }^{6}$

White crystalline solid, m. p.: $42-44{ }^{\circ} \mathrm{C} ;{ }^{1} \mathrm{H}$ NMR $\left(400 \mathrm{MHz}, 293 \mathrm{~K}, \mathrm{CDCl}_{3}\right): \delta=3.25-3.17(\mathrm{~m}, 2 \mathrm{H}), 3.04-3.02(\mathrm{~m}, 3 \mathrm{H})$, 

2.98-2.96 ppm (m, 3H); ${ }^{13} \mathrm{C}$ NMR $\left(100 \mathrm{MHz}, 293 \mathrm{~K}, \mathrm{CDCl}_{3}\right): \delta=163.1(\mathrm{q}, J=3.5 \mathrm{~Hz}), 124.1(\mathrm{q}, J=276.7 \mathrm{~Hz}), 38.4(\mathrm{q}, J=$ 29.1 Hz), 37.7, 35.5 ppm; ${ }^{19} \mathrm{~F}$ NMR (376 MHz, $\left.293 \mathrm{~K}, \mathrm{CDCl}_{3}\right): \delta=-62.4$ ppm (m); IR (film): $\widetilde{v}=2983,2953,1655,1432,1356$, 1275, 1171, 1119, 1104, 929, 854, $824 \mathrm{~cm}^{-1}$; HRMS (ESI): $m / z$ calculated for $\mathrm{C}_{5} \mathrm{H}_{8} \mathrm{~F}_{3} \mathrm{NONa}[\mathrm{M}+\mathrm{Na}]^{+}: 178.0446$, found: 178.0450 .

\section{2,3,3,3-Tetrafluoro- $N, N$-dimethylpropanamide (1bg).}

2,3,3,3-Tetrafluoropropionic acid ( $0.5 \mathrm{~g}, 3.4 \mathrm{mmol}, 1.0$ equiv.), triethylamine (0.95 mL, $6.8 \mathrm{mmol}, 2.0 \mathrm{equiv}$.), and $\mathrm{T} 3 \mathrm{P}^{\circledR}$ solution (2.7 $\mathrm{g}$ of a $50 \%$ solution in ethyl acetate, $4.3 \mathrm{mmol}, 1.25$ equiv.) were subsequently added to a solution of dimethylamine (2.0 M in THF, $1.8 \mathrm{~mL}, 3.6 \mathrm{mmol}, 1.05$ equiv.) $\left(2.25 \mathrm{~g}, 15.4 \mathrm{mmol}, 1.0 \mathrm{equiv}\right.$.) at $0{ }^{\circ} \mathrm{C}$. The reaction mixture was stirred at ambient temperature for $150 \mathrm{~min}$ before it was diluted with saturated aqueous $\mathrm{NaHCO}_{3}$ solution and extracted three times with ethyl acetate; the combined organic extracts were dried over $\mathrm{Na}_{2} \mathrm{SO}_{4}$, filtered and evaporated. The residue was purified by flash column chromatography (hexane/ethyl acetate, 3:1) to yield amide $\mathbf{1 b g}$ as a clear colorless oil (155 $\mathrm{mg}, 27 \%)$.

${ }^{1} \mathrm{H}$ NMR $\left(400 \mathrm{MHz}, 300 \mathrm{~K}, \mathrm{CDCl}_{3}\right): \delta=5.42(\mathrm{dq}, J=46.3,6.3 \mathrm{~Hz}), 3.13-3.11(\mathrm{~m}, 3 \mathrm{H}), 3.07-3.02$ ppm (m, 3H); ${ }^{13} \mathrm{C}$ NMR $\left(100 \mathrm{MHz}, 300 \mathrm{~K}, \mathrm{CDCl}_{3}\right): \delta=160.7(\mathrm{~d}, J=19.5 \mathrm{~Hz}), 121.0(\mathrm{qd}, J=282.0,26.1 \mathrm{~Hz}), 84.2(\mathrm{dq}, J=198.2,34.2 \mathrm{~Hz}), 36.6(\mathrm{dq}, J=$ 7.3, $1.4 \mathrm{~Hz}), 36.4$ ppm; ${ }^{19} \mathrm{~F}$ NMR (376 MHz, $\left.300 \mathrm{~K}, \mathrm{CDCl}_{3}\right): \delta=-75.5$ (s, 3F), -199.0 ppm (m, 1F); IR (film): $\widetilde{v}=2952,1680$, 1507, 1426, 1409, 1362, 1275, 1185, 1132, 983, 872 cm-1; HRMS (ESI): m/z calculated for $\mathrm{C}_{5} \mathrm{H}_{7} \mathrm{~F}_{4} \mathrm{NONa}[\mathrm{M}+\mathrm{Na}]^{+}:$196.0356, found: 196.0354 .

\section{4-2. Preparation of $N$-Boc and $N-C B z$ imines.}

All aromatic ${ }^{7}$ and aliphatic ${ }^{8} \mathrm{~N}$-Boc imines used herein $(\mathbf{2 a}, \mathbf{2} \mathbf{c}-\mathbf{2} \mathbf{q}$ and $\mathbf{2} \mathbf{r}-\mathbf{2 t})$ are literature-known and were prepared according to the general procedures cited.

All aromatic $N-C b z$ imines used herein $(\mathbf{2 b}, \mathbf{2} \mathbf{u}-\mathbf{2 z a})$ are literature-known and were prepared according to the general procedures cited. ${ }^{9}$

\section{4-3. Direct catalytic asymmetric Mannich-type reaction of $\alpha-\mathrm{CF}_{3}$ amide 1a.}

Procedure A:

A flame-dried $20 \mathrm{~mL}$ test tube equipped with a magnetic stirring bar and a 3-way glass stopcock was charged with $\left[\mathrm{Cu}\left(\mathrm{CH}_{3} \mathrm{CN}\right)_{4}\right] \mathrm{PF}_{6}(3.7 \mathrm{mg}, 0.01 \mathrm{mmol}, 10 \mathrm{~mol} \%)$ and $(R)-(-)-2,2$ '-bis[di(3,5-diisopropyl-4-dimethylaminophenyl)phosphine]-6,6'-dimethoxy-1,1'-biphenyl (DIPA-MeO-BIPHEP) (13.1 mg, $0.012 \mathrm{mmol}, 12 \mathrm{~mol} \%$ ) in a glove box under Ar atmosphere. Afterwards, the test tube was removed from the glove box and evacuated for 15 min. Then, it was backfilled with Ar and anhydrous THF $(0.2 \mathrm{~mL}, 0.33 \mathrm{M})$ was added at room temperature. The resulting clear colorless solution was stirred for $1 \mathrm{~h}$ before 1-(2,3-dihydro-1H-pyrrolo[2,3-b]pyridin-1-yl)-3,3,3-trifluoropropan-1-one (1a) (23.0 mg, 0.1 mmol, 1.0 equiv.), the corresponding $N$-Boc or $N$-Cbz imine (2a-2t) (0.2 mmol, 2.0 equiv.) and Barton's base (a $0.1 \mathrm{M}$ stock solution of Barton's base was prepared by diluting the commercial pure amine with the appropriate 

amount of freshly dried anhydrous THF and used for a maximum time period of 2 weeks before it was discarded; 0.1 $\mathrm{M}$ in THF, $0.1 \mathrm{~mL}, 0.01 \mathrm{mmol}, 10 \mathrm{~mol} \%$ ) were subsequently added. The reaction mixture was stirred at ambient temperature until the complete consumption of the amide starting material was observed by TLC analysis (<24 h). The diastereomeric ratio and the enantiomeric excess of the target material were determined by HPLC analysis of an aliquot, which was taken from the reaction mixture and filtered through a short pad of silica; the diastereomeric ratio is reported if it is worse than 20:1. Then, the reaction mixture was loaded directly onto a silica gel column and eluted with a suitable solution of $n$-hexane and ethyl acetate. Evaporation of solvent afforded the pure product.

Note that the NMR spectra of the Mannich products were recorded at elevated temperature (usually $\left.70{ }^{\circ} \mathrm{C}\right)$ in DMSO-d6, as rotamers were observed in $\mathrm{CDCl}_{3}$ at room temperature. ${ }^{10}$

\section{tert-Butyl ((1S,2S)-2-(2,3-dihydro-1H-pyrrolo[2,3-b]pyridine-1-carbonyl)-3,3,3-trifluoro-1-phenylpropyl)carbamate}

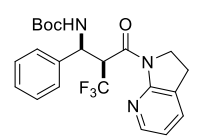

(3a).

The reaction was performed according to procedure A using $\left[\mathrm{Cu}\left(\mathrm{CH}_{3} \mathrm{CN}\right)_{4}\right] \mathrm{PF}_{6}(1.9 \mathrm{mg}, 0.005 \mathrm{mmol}, 5$ mol\%), (R)-(-)-2,2'-bis[di(3,5-diisopropyl-4-dimethylaminophenyl)phosphine]-6,6'-dimethoxy-1,1'-biphenyl (DIPAMeO-Biphep) (6.5 mg, $0.006 \mathrm{mmol}, 6 \mathrm{~mol} \%)$, anhydrous THF (0.15 mL, $0.5 \mathrm{M})$, N-Boc imine $2 \mathrm{a}$ (41.0 mg), and Barton's base $(0.1 \mathrm{M}$ in THF, $50 \mu \mathrm{L}, 0.005 \mathrm{mmol}, 5 \mathrm{~mol} \%) .40 .8 \mathrm{mg}(94 \%)$ of the desired pure product 3a were obtained after purification by flash column chromatography (hexane/ethyl acetate, 3:1).

White solid, m. p.: 161-163 ${ }^{\circ} \mathrm{C}$; ${ }^{1} \mathrm{H}$ NMR (400 MHz, $\left.343 \mathrm{~K}, \mathrm{DMSO}-\mathrm{d}_{6}\right): \delta=8.16(\mathrm{~d}, J=4.4 \mathrm{~Hz}, 1 \mathrm{H}), 7.66$ (brs, $\left.1 \mathrm{H}\right), 7.56(\mathrm{dd}$, $J=7.4,1.3 \mathrm{~Hz}, 1 \mathrm{H}), 7.24(\mathrm{dd}, J=7.7,1.3 \mathrm{~Hz}, 2 \mathrm{H}), 7.12-7.01(\mathrm{~m}, 4 \mathrm{H}), 6.44-6.35(\mathrm{~m}, 1 \mathrm{H}), 5.27(\mathrm{t}, J=10.2 \mathrm{~Hz}, 1 \mathrm{H}), 3.77(\mathrm{ddd}$, $J=14.9,7.7,4.5 \mathrm{~Hz}, 1 \mathrm{H}), 3.45(\mathrm{ddd}, J=11.7,11.0,5.1 \mathrm{~Hz}, 1 \mathrm{H}), 2.84(\mathrm{ddd}, J=15.9,10.5,5.2 \mathrm{~Hz}, 1 \mathrm{H}), 2.57(\mathrm{ddd}, J=17.4$, 10.4, 7.2 Hz, 1H), 1.35 ppm (s, 9H); $\left.{ }^{13} \mathrm{C} \mathrm{NMR} \mathrm{(150} \mathrm{MHz,} 343 \mathrm{~K}, \mathrm{DMSO}-d_{6}\right): \delta=163.3$ (br), 154.2 (br), $153.9,145.0,139.1$, 134.0, 127.3, 127.1, 127.0(5), 126.1, 124.8 (q, J = 282.2 Hz), 118.8, 77.9, 52.9 (br), 50.8 (q, J = 23.1 Hz), 45.5, 27.9, 23.0 ppm; ${ }^{19} \mathrm{~F}$ NMR (376 MHz, $\left.343 \mathrm{~K}, \mathrm{DMSO}-d_{6}\right): \delta=-61.5 \mathrm{ppm}(\mathrm{m}) ; \mathrm{IR}$ (film): $\widetilde{v}=3341,3066,3007,2973,2932,1705,1662,1594$, 1507, 1475, 1425, 1366, 1263, 1152, 1124, 1022, 866, 786, $755 \mathrm{~cm}^{-1}$; HRMS (ESI): m/z calculated for $\mathrm{C}_{22} \mathrm{H}_{24} \mathrm{~F}_{3} \mathrm{~N}_{3} \mathrm{O}_{3} \mathrm{Na}$ $[\mathrm{M}+\mathrm{Na}]^{+}:$458.1662, found: 458.1654; $[\alpha]_{D}^{24}=-62.6\left(c=1.00, \mathrm{CHCl}_{3}, 98 \%\right.$ ee sample); HPLC analysis (CHIRALPAK IA $(\phi=0.46 \mathrm{~cm} \times 25 \mathrm{~cm}), 2$-propanol $/ n$-hexane $=1 / 9$, flow rate $=1.0 \mathrm{~mL} / \mathrm{min}$, detection at $254 \mathrm{~nm}, \mathrm{tR}=12.0 \mathrm{~min}(\mathrm{minor})$, $13.7 \min ($ major$))$ :
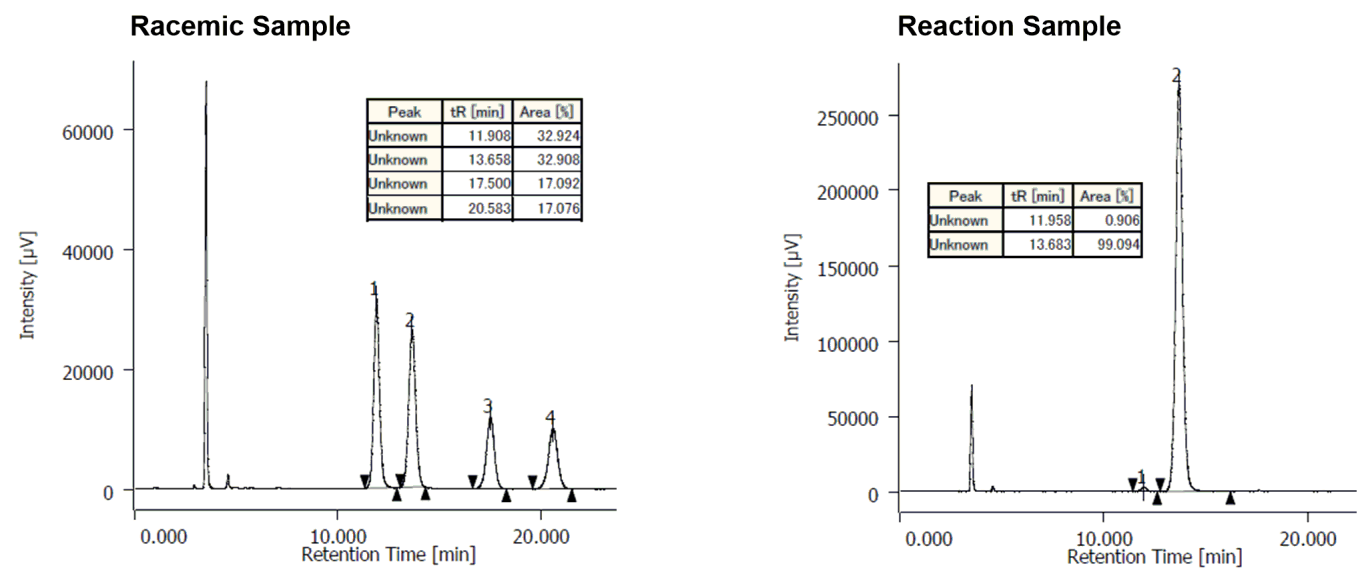

tert-Butyl ((1R,2R)-2-(2,3-dihydro-1H-pyrrolo[2,3-b]pyridine-1-carbonyl)-3,3,3-trifluoro-1-phenylpropyl)carbamate

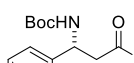
(SI-3).

The reaction was performed according to procedure A using $\left[\mathrm{Cu}\left(\mathrm{CH}_{3} \mathrm{CN}\right)_{4}\right] \mathrm{PF}_{6}(1.9 \mathrm{mg}, 0.005 \mathrm{mmol}, 5$ mol\%), the corresponding enantiomeric ligand (S)-(+)-2,2'-bis[di(3,5-diisopropyl-4-dimethylaminophenyl)phosphine]6,6'-dimethoxy-1,1'-biphenyl (DIPA-MeO-Biphep) (6.5 mg, $0.006 \mathrm{mmol}, 6 \mathrm{~mol} \%)$, anhydrous THF (0.15 mL, $0.5 \mathrm{M})$, $N$-Boc imine 2a (41.0 mg), and Barton's base (0.1 M in THF, $50 \mu \mathrm{L}, 0.005 \mathrm{mmol}, 5 \mathrm{~mol} \%) .41 .0 \mathrm{mg}(94 \%)$ of the desired pure product SI-3 were obtained after purification by flash column chromatography (hexane/ethyl acetate, 2:1).

White solid, m. p.: $167-168{ }^{\circ} \mathrm{C} ;{ }^{1} \mathrm{H}$ NMR (400 MHz, $343 \mathrm{~K}$, DMSO- $\left.d_{6}\right): \delta=8.17-8.14$ (m, 1H), 7.66-7.48 (m, 2H), 7.26-7.23 (m, 2H), 7.12-7.05 (m, 3H), 7.03 (dd, J= 7.4, $5.1 \mathrm{~Hz}, 1 \mathrm{H}), 6.44-6.35(\mathrm{~m}, 1 \mathrm{H}), 5.26(\mathrm{brt}, J=10.1 \mathrm{~Hz}, 1 \mathrm{H}), 3.78(\mathrm{ddd}, J=12.1$, 10.5, $7.4 \mathrm{~Hz}, 1 \mathrm{H}), 3.45$ (ddd, $J=12.0,10.7,5.2 \mathrm{~Hz}, 1 \mathrm{H}), 2.89-2.81(\mathrm{~m}, 1 \mathrm{H}), 2.62-2.54(\mathrm{~m}, 1 \mathrm{H}), 1.35 \mathrm{ppm}(\mathrm{s}, 9 \mathrm{H}) ;{ }^{13} \mathrm{C}$ NMR (125 MHz, 343 K, DMSO-d6): $\delta=163.3$ (br), 154.1 (br), 153.9, 145.0, 139.1, 134.0, 127.3, 127.1, 127.0, 126.1, 124.8 (q, $J=$ $281.9 \mathrm{~Hz}), 118.8,77.9,52.9(\mathrm{br}), 50.7(\mathrm{q}, J=23.6 \mathrm{~Hz}), 45.4,27.8,23.0$ ppm; ${ }^{19} \mathrm{~F}$ NMR $\left(376 \mathrm{MHz}, 343 \mathrm{~K}, \mathrm{DMSO}-d_{6}\right): \delta=-61.7$ ppm (s); IR (film): $\widetilde{v}=3341,3062,3007,2977,1705,1662,1594,1507,1476,1426,1366,1264,1152,1124,757 \mathrm{~cm}^{-1}$; HRMS (ESI): $m / z$ calculated for $\mathrm{C}_{22} \mathrm{H}_{24} \mathrm{~F}_{3} \mathrm{~N}_{3} \mathrm{O}_{3} \mathrm{Na}[\mathrm{M}+\mathrm{Na}]^{+}: 458.1662$, found: 458.1659; $[\alpha]_{D}^{26}=+64.5\left(c=0.65, \mathrm{CHCl}_{3}, 97 \% e e\right.$ sample); HPLC analysis (CHIRALPAK IA $(\phi=0.46 \mathrm{~cm} \times 25 \mathrm{~cm}$ ), 2-propanol $/ n$-hexane $=1 / 9$, flow rate $=1.0 \mathrm{~mL} / \mathrm{min}$, detection at $254 \mathrm{~nm}, \mathrm{t}_{\mathrm{R}}=12.0 \mathrm{~min}$ (major), $13.8 \mathrm{~min}$ (minor)):
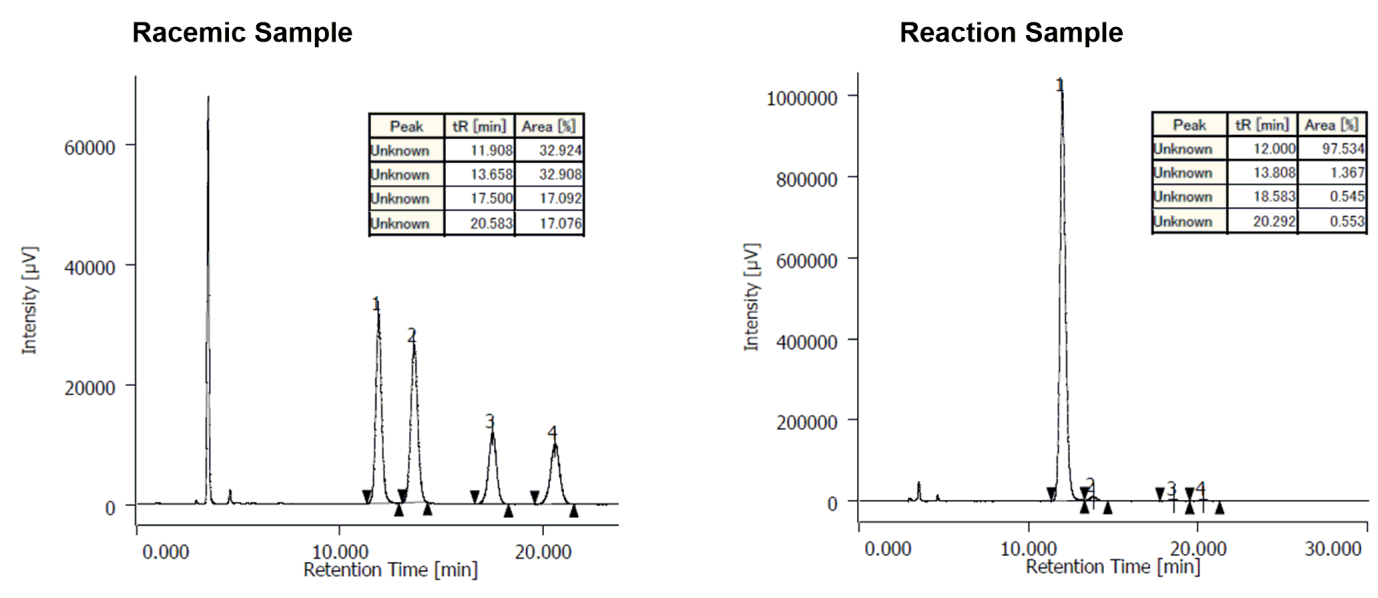

Benzyl ((1S,2S)-2-(2,3-dihydro-1H-pyrrolo[2,3-b]pyridine-1-carbonyl)-3,3,3-trifluoro-1-phenylpropyl)carbamate (3b).

The reaction was performed according to procedure A using $\left.\left[\mathrm{Cu}_{(\mathrm{CH}} \mathrm{CH}_{3} \mathrm{CN}\right)_{4}\right] \mathrm{PF}_{6}(1.9 \mathrm{mg}, 0.005 \mathrm{mmol}, 5$ mol\%), (R)-(-)-2,2'-bis[di(3,5-diisopropyl-4-dimethylaminophenyl)phosphine]-6,6'-dimethoxy1,1'-biphenyl (DIPA-MeO-Biphep) (6.5 mg, $0.006 \mathrm{mmol}, 6 \mathrm{~mol} \%)$, anhydrous THF (0.15 mL, $0.5 \mathrm{M}), \mathrm{N}$-Cbz imine 2b (47.8 mg), and Barton's base (0.1 M in THF, $50 \mu \mathrm{L}, 0.005 \mathrm{mmol}, 5 \mathrm{~mol} \%) .44 .1 \mathrm{mg}$ (94\%) of the desired pure product $3 \mathbf{b}$ were obtained after purification by flash column chromatography (hexane/ethyl acetate, 2:1).

White amorphous solid; ${ }^{1} \mathrm{H}$ NMR (400 MHz, $343 \mathrm{~K}$, DMSO- $\left.d_{6}\right): \delta=8.16-8.14(\mathrm{~m}, 1 \mathrm{H}), 8.09$ (brs, $\left.1 \mathrm{H}\right), 7.57$ (ddd, $J=7.4$, 2.7, $1.2 \mathrm{~Hz}, 1 \mathrm{H}), 7.35-7.24(\mathrm{~m}, 7 \mathrm{H}), 7.14-7.06(\mathrm{~m}, 3 \mathrm{H}), 7.03(\mathrm{dd}, J=7.4,5.1 \mathrm{~Hz}, 1 \mathrm{H}), 6.50-6.41(\mathrm{~m}, 1 \mathrm{H}), 5.33(\mathrm{t}, J=10.4 \mathrm{~Hz}$, 1H), $5.06(\mathrm{~d}, J=12.7 \mathrm{~Hz}, 1 \mathrm{H}), 4.99(\mathrm{~d}, J=12.7 \mathrm{~Hz}, 1 \mathrm{H}), 3.79(\mathrm{ddd}, J=12.1,10.5,7.3 \mathrm{~Hz}, 1 \mathrm{H}), 3.46(\mathrm{ddd}, J=12.0,10.7,5.2$ $\mathrm{Hz}, 1 \mathrm{H}), 2.86(\mathrm{ddd}, J=16.7,10.5,5.2 \mathrm{~Hz}, 1 \mathrm{H}), 2.59 \mathrm{ppm}(\mathrm{ddd}, J=16.8,10.5,7.3 \mathrm{~Hz}, 1 \mathrm{H}) ;{ }^{13} \mathrm{C} \mathrm{NMR}(125 \mathrm{MHz}, 343 \mathrm{~K}$, 

DMSO-d6): $\delta=163.1,154.8$ (br), 153.8, 145.0, 138.8, 136.7, 134.1, 127.7, 127.4, 127.3, 127.2, 127.1, 127.0(7), 126.2, 124.7 (q, J $=282.1 \mathrm{~Hz}), 118.8,65.2,53.5(\mathrm{br}), 50.6(\mathrm{q}, J=23.9 \mathrm{~Hz}), 45.5,23.9$ ppm; ${ }^{19} \mathrm{~F}$ NMR $\left(376 \mathrm{MHz}, 343 \mathrm{~K}, \mathrm{DMSO}-d_{6}\right): \delta=-61.7$ ppm (s); IR (film): $\widetilde{v}=3324$ (br), 3066, 3032, 2959, 2918, 1704, 1660, 1594, 1540, 1426, 1352, 1262, 1152, 1125, 1026, 770 $\mathrm{cm}^{-1}$; HRMS (ESI): $m / z$ calculated for $\mathrm{C}_{25} \mathrm{H}_{23} \mathrm{~F}_{3} \mathrm{~N}_{3} \mathrm{O}_{3}[\mathrm{M}+\mathrm{H}]^{+}: 470.1686$, found: $470.1681 ;[\alpha]_{D}^{26}=-21.0\left(c=0.40, \mathrm{CHCl}_{3}\right.$, 98\% ee sample); HPLC analysis (CHIRALPAK IA $(\phi=0.46 \mathrm{~cm} \times 25 \mathrm{~cm})$, 2-propanol $/ n$-hexane $=1 / 9$, flow rate $=1.0$ $\mathrm{mL} / \mathrm{min}$, detection at $254 \mathrm{~nm}, \mathrm{t}=33.6 \mathrm{~min}$ (minor), $38.8 \mathrm{~min}$ (major)):
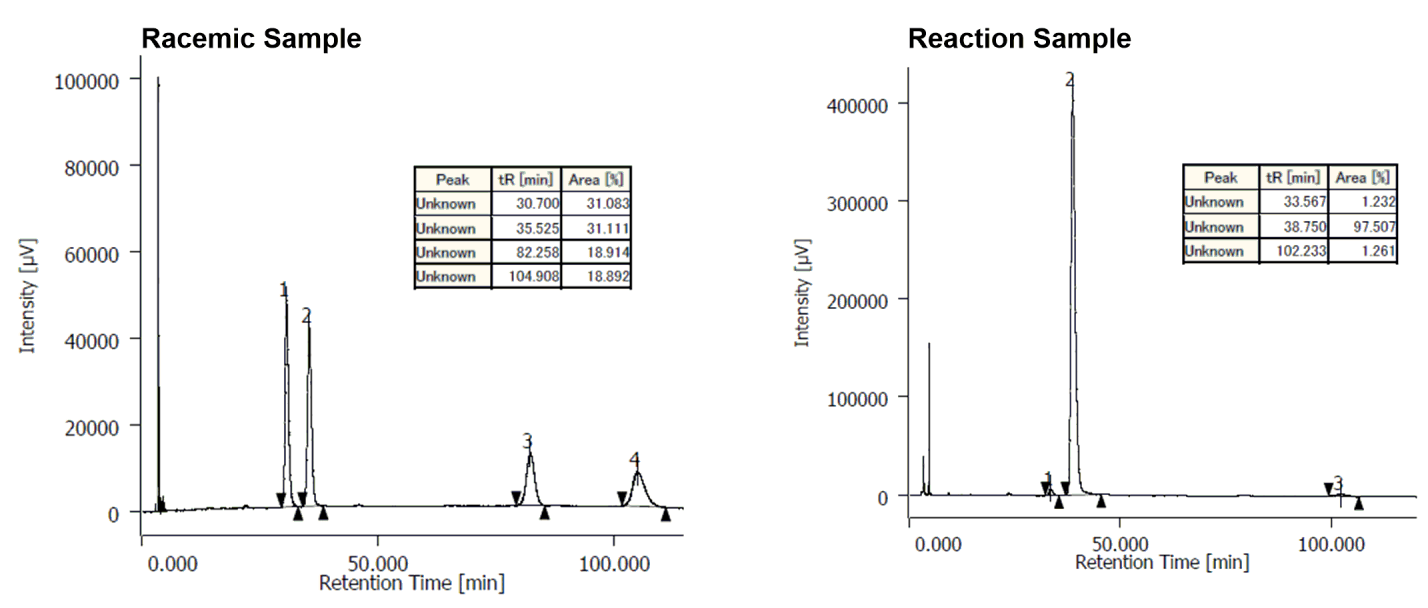

tert-Butyl (1S,2S)-2-(2,3-dihydro-1H-pyrrolo[2,3-b]pyridine-1-carbonyl)-3,3,3-trifluoro-1-(naphthalen-2-yl)propyl)carbamate (3c).

The reaction was performed according to procedure A using N-Boc imine $2 \mathrm{c}(51.1 \mathrm{mg})$ to afford 45.2 (hexane/ethyl acetate, 2:1).

White solid, m. p.: $197^{\circ} \mathrm{C} ;{ }^{1} \mathrm{H}$ NMR (400 MHz, $343 \mathrm{~K}$, DMSO-d6): $\delta=8.20$ (d, J=4.6 Hz, 1H), 7.82-7.73 (m, 3H), 7.62-7.58 $(\mathrm{m}, 2 \mathrm{H}), 7.44-7.40(\mathrm{~m}, 4 \mathrm{H}), 7.01(\mathrm{dd}, J=6.9,5.5 \mathrm{~Hz}, 1 \mathrm{H}), 6.56-6.47(\mathrm{~m}, 1 \mathrm{H}), 5.44(\mathrm{t}, J=9.2 \mathrm{~Hz}, 1 \mathrm{H}), 3.69(\mathrm{td}, J=10.9,7.9$ $\mathrm{Hz}, 1 \mathrm{H}), 3.33(\mathrm{td}, J=11.3,4.7 \mathrm{~Hz}, 1 \mathrm{H}), 2.69(\mathrm{ddd}, J=16.2,10.7,5.2 \mathrm{~Hz}, 1 \mathrm{H}), 2.18-2.10(\mathrm{~m}, 1 \mathrm{H}), 1.35 \mathrm{ppm}(\mathrm{s}, 9 \mathrm{H}) ;{ }^{13} \mathrm{C} \mathrm{NMR}$ (150 MHz, $\left.343 \mathrm{~K}, \mathrm{DMSO}-d_{6}\right): \delta=163.3$ (q, $\left.J=3.4 \mathrm{~Hz}\right), 154.2$ (br), 153.8, 145.0, 136.5, 133.9, 132.0, 131.9(5), 127.2, 126.9(4), 126.9, 126.1, 126.0, 125.7, 125.6(5), 125.1, 124.8 (q, $J=281.8 \mathrm{~Hz}), 118.8,77.9,53.1$ (br), 50.7 (q, J = 22.6 Hz), 45.4, $27.8,22.8$ ppm; ${ }^{19} \mathrm{~F}$ NMR (376 MHz, $\left.343 \mathrm{~K}, \mathrm{DMSO}-d_{6}\right): \delta=-61.5$ ppm (m); IR (film): $\widetilde{v}=3341,3059,3011,2976,2928,1705,1661$, 1594, 1507, 1476, 1426, 1366, 1265, 1152, 1021, 859, 786, $753 \mathrm{~cm}^{-1}$; HRMS (ESI): m/z calculated for $\mathrm{C}_{26} \mathrm{H}_{26} \mathrm{~F}_{3} \mathrm{~N}_{3} \mathrm{O}_{3} \mathrm{Na}$ $[\mathrm{M}+\mathrm{Na}]^{+}:$508.1818, found: 509.1801; $[\alpha]_{D}^{24}=-112.0\left(c=0.65, \mathrm{CHCl}_{3}, 95 \%\right.$ ee sample); HPLC analysis (CHIRALPAK IA $(\phi=0.46 \mathrm{~cm} \times 25 \mathrm{~cm}), 2$-propanol $/ n$-hexane $=1 / 9$, flow rate $=1.0 \mathrm{~mL} / \mathrm{min}$, detection at $254 \mathrm{~nm}, \mathrm{t}=16.3 \mathrm{~min}(\mathrm{minor})$, $18.7 \min$ (major)): 

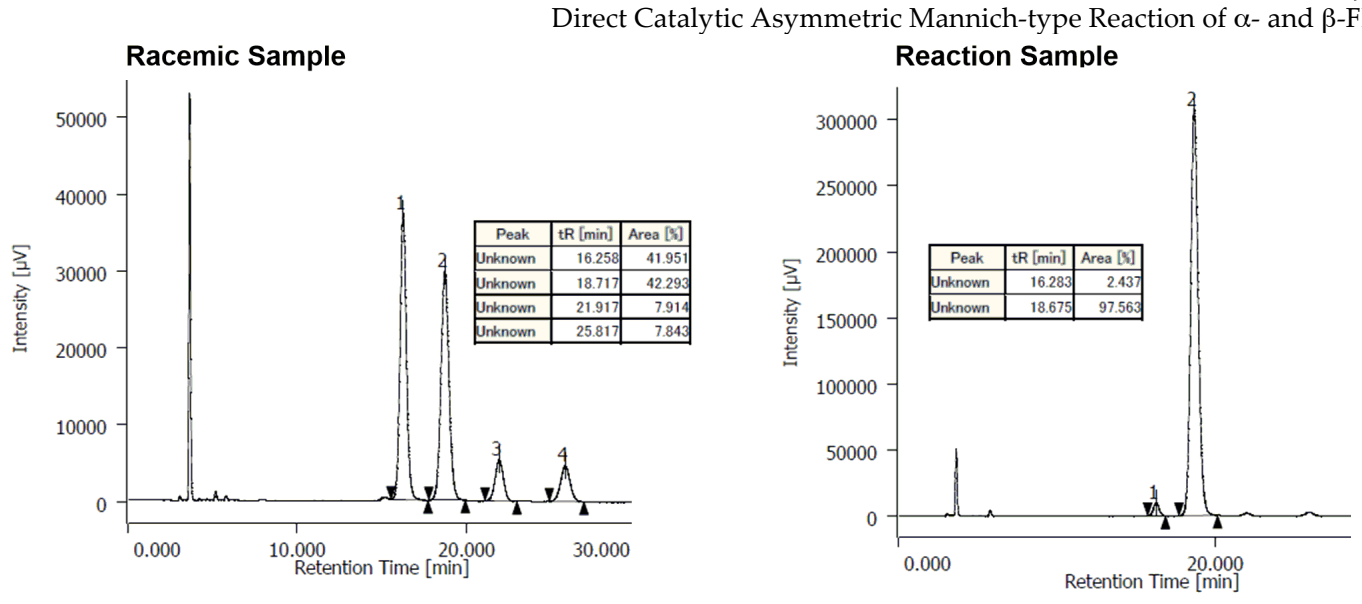

tert-Butyl ((1S,2S)-2-(2,3-dihydro-1H-pyrrolo[2,3-b]pyridine-1-carbonyl)-3,3,3-trifluoro-1-(meta-tolyl)propyl)-

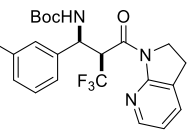
carbamate (3d).

The reaction was performed according to procedure A using N-Boc imine $2 \mathbf{d}(43.9 \mathrm{mg}$ ) to afford 43.2 $\mathrm{mg}(96 \%)$ of the desired pure product 3d after purification by flash column chromatography (hexane/ethyl acetate, 3:1). White solid, m. p.: $176-178{ }^{\circ} \mathrm{C} ;{ }^{1} \mathrm{H}$ NMR (400 MHz, $\left.343 \mathrm{~K}, \mathrm{DMSO}-\mathrm{d}_{6}\right): \delta=8.17$ (d, J=4.4 Hz, $\left.1 \mathrm{H}\right), 7.61$ (brs, $\left.1 \mathrm{H}\right), 7.57$ (d, J $=7.3 \mathrm{~Hz}, 1 \mathrm{H}), 7.05-6.89(\mathrm{~m}, 5 \mathrm{H}), 6.41-6.32(\mathrm{~m}, 1 \mathrm{H}), 5.20$ (brt, $J=9.5 \mathrm{~Hz}, 1 \mathrm{H}), 3.74(\mathrm{ddd}, J=11.6,10.4,7.5 \mathrm{~Hz}, 1 \mathrm{H}), 3.43$ (td, $J=11.3,4.9 \mathrm{~Hz}, 1 \mathrm{H}), 2.82(\mathrm{ddd}, J=15.9,10.9,4.9 \mathrm{~Hz}, 1 \mathrm{H}), 2.57-2.50(\mathrm{~m}, 1 \mathrm{H}), 2.02(\mathrm{~s}, 3 \mathrm{H}), 1.35 \mathrm{ppm}(\mathrm{s}, 9 \mathrm{H}) ;{ }^{13} \mathrm{C} \mathrm{NMR}$ (150 MHz, 343 K, DMSO-d6): $\delta=163.4$ (q, J = 2.9 Hz), 154.1 (br), 153.9, 145.0, 138.9, 136.6, 134.0, 127.8, 127.6, 127.2, 126.2, $124.8(q, J=281.8 \mathrm{~Hz}), 124.2,118.8,77.8,53.0(\mathrm{br}), 50.7(\mathrm{q}, J=23.1 \mathrm{~Hz}), 45.5,27.9,23.0,20.3$ ppm; ${ }^{19} \mathrm{~F} \mathrm{NMR}(376 \mathrm{MHz}, 343$ K, DMSO-d6): $\delta=-61.5$ ppm (m); IR (film): $\widetilde{v}=3344,3059,3011,2977,2928,1705,1663,1593,1506,1476,1443,1426,1366$,

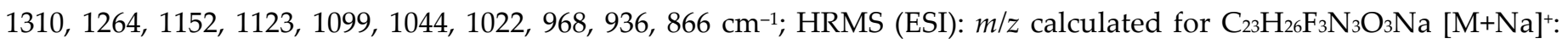
472.1818, found: 472.1812; $[\alpha]_{D}^{24}=-67.1\left(c=1.75, \mathrm{CHCl}_{3}, 97 \%\right.$ ee sample); HPLC analysis (CHIRALPAK IA $(\phi=0.46 \mathrm{~cm}$ x $25 \mathrm{~cm}$ ), 2-propanol $/ n$-hexane $=1 / 9$, flow rate $=1.0 \mathrm{~mL} / \mathrm{min}$, detection at $254 \mathrm{~nm}, \mathrm{t}$ = $10.6 \mathrm{~min}(\mathrm{minor}), 12.1 \mathrm{~min}$ (major)):
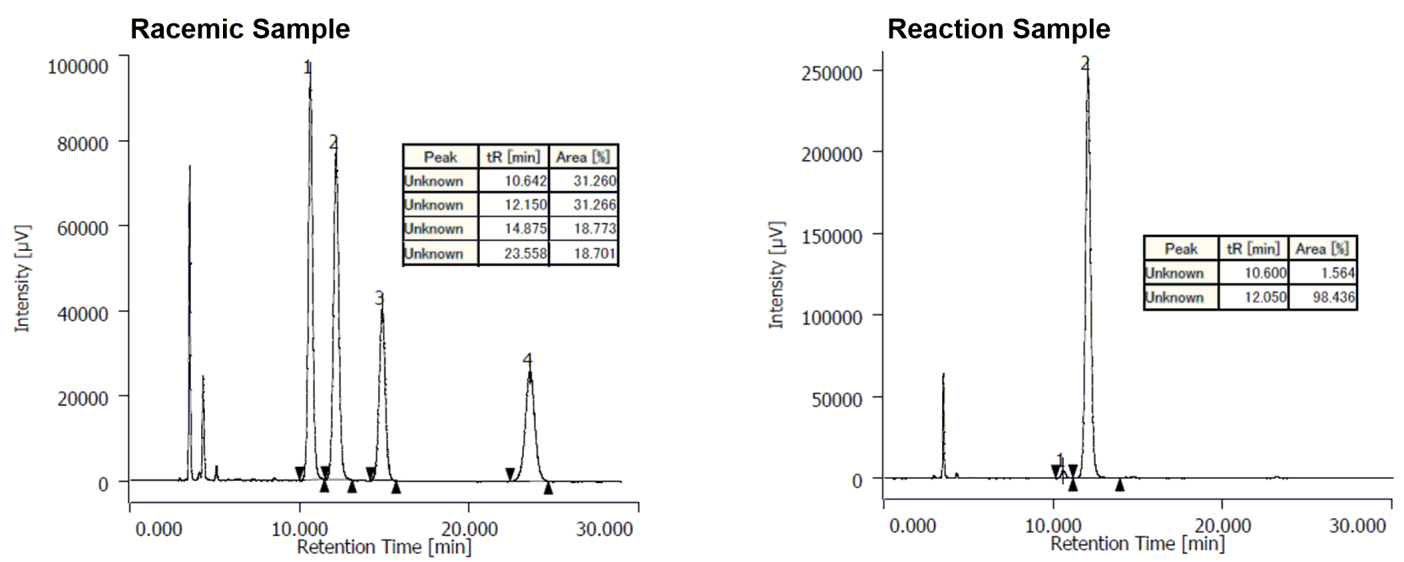

tert-Butyl ((1S,2S)-2-(2,3-dihydro-1H-pyrrolo[2,3-b]pyridine-1-carbonyl)-3,3,3-trifluoro-1-(para-tolyl)propyl)-

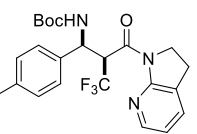
carbamate (3e).

The reaction was performed according to procedure A using $\left[\mathrm{Cu}\left(\mathrm{CH}_{3} \mathrm{CN}\right)_{4}\right] \mathrm{PF}_{6}(1.9 \mathrm{mg}, 0.005 \mathrm{mmol}, 5$ mol\%), (R)-(-)-2,2'-bis[di(3,5-diisopropyl-4-dimethylaminophenyl)phosphine]-6,6'-dimethoxy-1,1'-biphenyl (DIPAMeO-Biphep) (6.5 mg, $0.006 \mathrm{mmol}, 6 \mathrm{~mol} \%)$, anhydrous THF (0.15 mL, $0.5 \mathrm{M})$, N-Boc imine $2 \mathbf{e}$ (43.9 mg), and Barton's base $(0.1 \mathrm{M}$ in THF, $50 \mu \mathrm{L}, 0.005 \mathrm{mmol}, 5 \mathrm{~mol} \%) .41 .3 \mathrm{mg}(92 \%)$ of the desired pure product 3e were obtained after purification by flash column chromatography (hexane/ethyl acetate, 2:1).

White solid, m. p.: $191-193{ }^{\circ} \mathrm{C}$; ${ }^{1} \mathrm{H}$ NMR (400 MHz, $\left.343 \mathrm{~K}, \mathrm{DMSO}-\mathrm{d}_{6}\right): \delta=8.15$ (d, J = $\left.5.0 \mathrm{~Hz}, 1 \mathrm{H}\right), 7.58$ (brs, $\left.1 \mathrm{H}\right), 7.57$ (d, J $=7.3 \mathrm{~Hz}, 1 \mathrm{H}), 7.11(\mathrm{~d}, J=7.3 \mathrm{~Hz}, 2 \mathrm{H}), 7.05-7.01(\mathrm{~m}, 1 \mathrm{H}), 6.87$ (d, J = 7.6 Hz, 2H), 6.40-6.32 (m, $1 \mathrm{H}), 5.23(\mathrm{brt}, J=9.5 \mathrm{~Hz}$, 1H), 3.81-3.74 (m, 1H), 3.53-3.46 (m, 1H), 2.85 (ddd, J = 16.0, 10.6, $5.2 \mathrm{~Hz}, 1 \mathrm{H}), 2.62-2.54(\mathrm{~m}, 1 \mathrm{H}), 2.15(\mathrm{~s}, 3 \mathrm{H}), 1.36 \mathrm{ppm}$ (s, 9H); ${ }^{13} \mathrm{C}$ NMR (150 MHz, $\left.343 \mathrm{~K}, \mathrm{DMSO}-d_{6}\right): \delta=163.3$ (br), 154.1 (br), 153.9, 145.0, 136.2, 136.1(9), 133.9, 127.8, 126.9, 126.2, $124.9(q, J=282.3 \mathrm{~Hz}), 118.8,77.8,52.6(\mathrm{br}), 50.8(\mathrm{q}, J=23.1 \mathrm{~Hz}), 45.5,27.9,23.0,20.1 \mathrm{ppm}$; ${ }^{19} \mathrm{~F}$ NMR $(376 \mathrm{MHz}, 343$ K, DMSO-d6): $\delta=-61.5$ ppm (s); IR (film): $\widetilde{v}=3344,3056,3011,2977,2928,2867,1706,1663,1594,1513,1476,1426,1366$, $1316,1263,1153,1123,1045,1021,967,897,867,819,785,756 \mathrm{~cm}^{-1} ;$ HRMS (ESI): $m / z$ calculated for $\mathrm{C}_{23} \mathrm{H}_{26} \mathrm{~F}_{3} \mathrm{~N}_{3} \mathrm{O}_{3} \mathrm{Na}_{2}$ $[\mathrm{M}+\mathrm{Na}]^{+}:$472.1818, found: $472.1816 ;[\alpha]_{D}^{24}=-72.9\left(c=1.13, \mathrm{CHCl}_{3}, 99 \%\right.$ ee sample); HPLC analysis (CHIRALPAK IE $(\phi=0.46 \mathrm{~cm} \times 25 \mathrm{~cm}), 2$-propanol $/ n$-hexane $=1 / 4$, flow rate $=1.0 \mathrm{~mL} / \mathrm{min}$, detection at $254 \mathrm{~nm}, \mathrm{t}=18.8 \mathrm{~min}(\mathrm{major}), 27.0$ $\min ($ minor)):
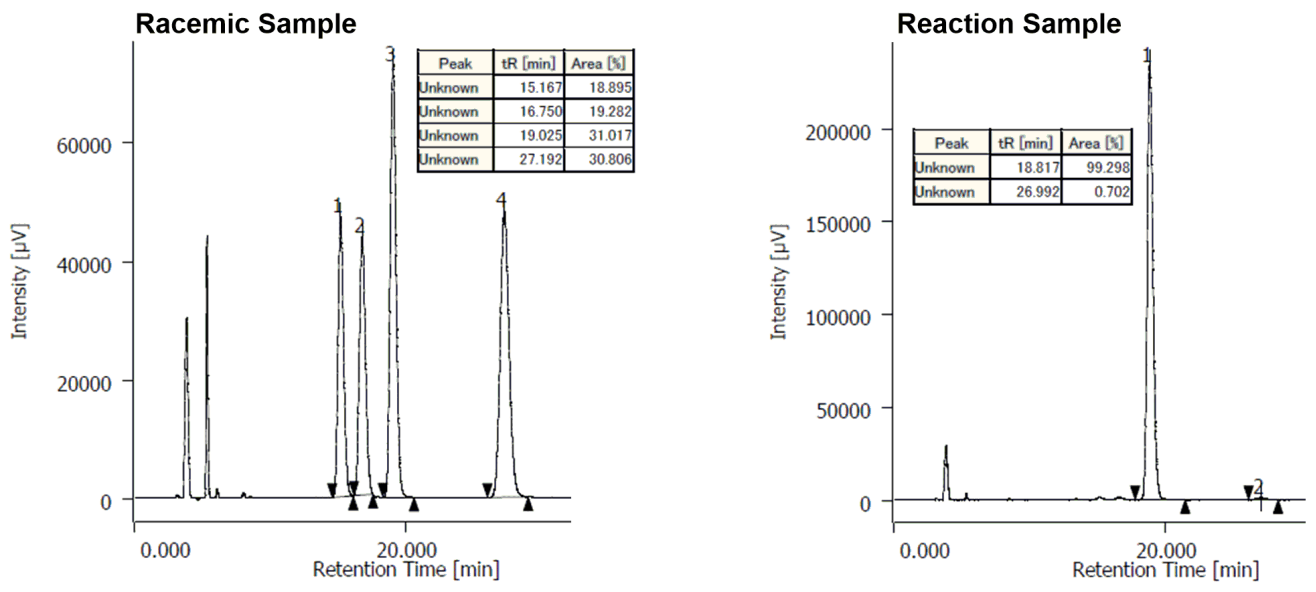

tert-Butyl ((1S,2S)-2-(2,3-dihydro-1H-pyrrolo[2,3-b]pyridine-1-carbonyl)-3,3,3-trifluoro-1-(3-vinylphenyl)propyl)-

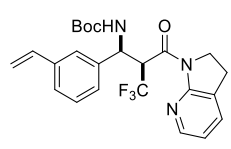
carbamate (3f).

The reaction was performed according to procedure A using $N$-Boc imine $2 \mathrm{f}(46.3 \mathrm{mg})$ to afford 42.4 mg $(92 \%)$ of the desired pure product $3 \mathbf{f}$ after purification by flash column chromatography (hexane/ethyl acetate, 3:1).

White solid, m. p.: $155-156{ }^{\circ} \mathrm{C} ;{ }^{1} \mathrm{H}$ NMR (400 MHz, $\left.343 \mathrm{~K}, \mathrm{DMSO}-\mathrm{d}_{6}\right): \delta=8.16$ (d, J = 5.0 Hz, $\left.1 \mathrm{H}\right), 7.67$ (brs, $\left.1 \mathrm{H}\right), 7.55$ (d, $J$ $=7.3 \mathrm{~Hz}, 1 \mathrm{H}), 7.38(\mathrm{~s}, 1 \mathrm{H}), 7.17(\mathrm{~d}, J=7.3 \mathrm{~Hz}, 1 \mathrm{H}), 7.12(\mathrm{~d}, J=7.8 \mathrm{~Hz}, 1 \mathrm{H}), 7.08-7.06(\mathrm{~m}, 1 \mathrm{H}), 7.04-7.00(\mathrm{~m}, 1 \mathrm{H}), 6.50-6.38$ $(\mathrm{m}, 2 \mathrm{H}), 5.52(\mathrm{~d}, J=17.6 \mathrm{~Hz}, 1 \mathrm{H}), 5.26(\mathrm{brt}, J=9.4 \mathrm{~Hz}, 1 \mathrm{H}), 5.09$ (d, J = 11.0, 1H), $3.76(\mathrm{ddd}, J=11.9,10.7,7.5 \mathrm{~Hz}, 1 \mathrm{H})$, 3.46-3.39 (m, 1H), $2.84(\mathrm{ddd}, J=15.9,10.4,5.2 \mathrm{~Hz}, 1 \mathrm{H}), 2.58-2.51$ (m, 1H), $1.36 \mathrm{ppm}(\mathrm{s}, 9 \mathrm{H}) ;{ }^{13} \mathrm{C} \mathrm{NMR}(150 \mathrm{MHz}, 343 \mathrm{~K}$, DMSO-d $): \delta=163.3(q, J=2.9 \mathrm{~Hz}), 154.2(\mathrm{br}), 153.8,145.0,139.4,136.6,135.9,134.0,127.6,126.8,126.1,125.2,124.8(\mathrm{q}, J=$ 

282.3 Hz), 124.6, 118.8, 113.7, 77.9, 52.9 (br), $50.7(q, J=24.6 \mathrm{~Hz}), 45.5,27.8,23.0 \mathrm{ppm}$; ${ }^{19} \mathrm{~F} \mathrm{NMR} \mathrm{(376} \mathrm{MHz,} \mathrm{343} \mathrm{K,}$ DMSO-d6): $\delta=-61.6$ ppm (s); IR (film): $\widetilde{v}=3337,3004,2977,2932,1704,1662,1594,1507,1476,1425,1366,1264,1153$, 1124, 1022, 910, $865 \mathrm{~cm}^{-1}$; HRMS (ESI): $m / z$ calculated for $\mathrm{C}_{24} \mathrm{H}_{26} \mathrm{~F}_{3} \mathrm{~N}_{3} \mathrm{O}_{3} \mathrm{Na}[\mathrm{M}+\mathrm{Na}]^{+}: 484.1818$, found: $484.1812 ;[\alpha]_{D}^{24}=$ -56.9 ( $c=1.08, \mathrm{CHCl}_{3}, 96 \%$ ee sample); HPLC analysis (CHIRALPAK IA $(\phi=0.46 \mathrm{~cm} \times 25 \mathrm{~cm}), 2$-propanol $/ n$-hexane $=$ $1 / 9$, flow rate $=1.0 \mathrm{~mL} / \mathrm{min}$, detection at $254 \mathrm{~nm}, \mathrm{t}_{\mathrm{R}}=11.4 \mathrm{~min}$ (minor), $13.6 \mathrm{~min}($ major $)$ ):
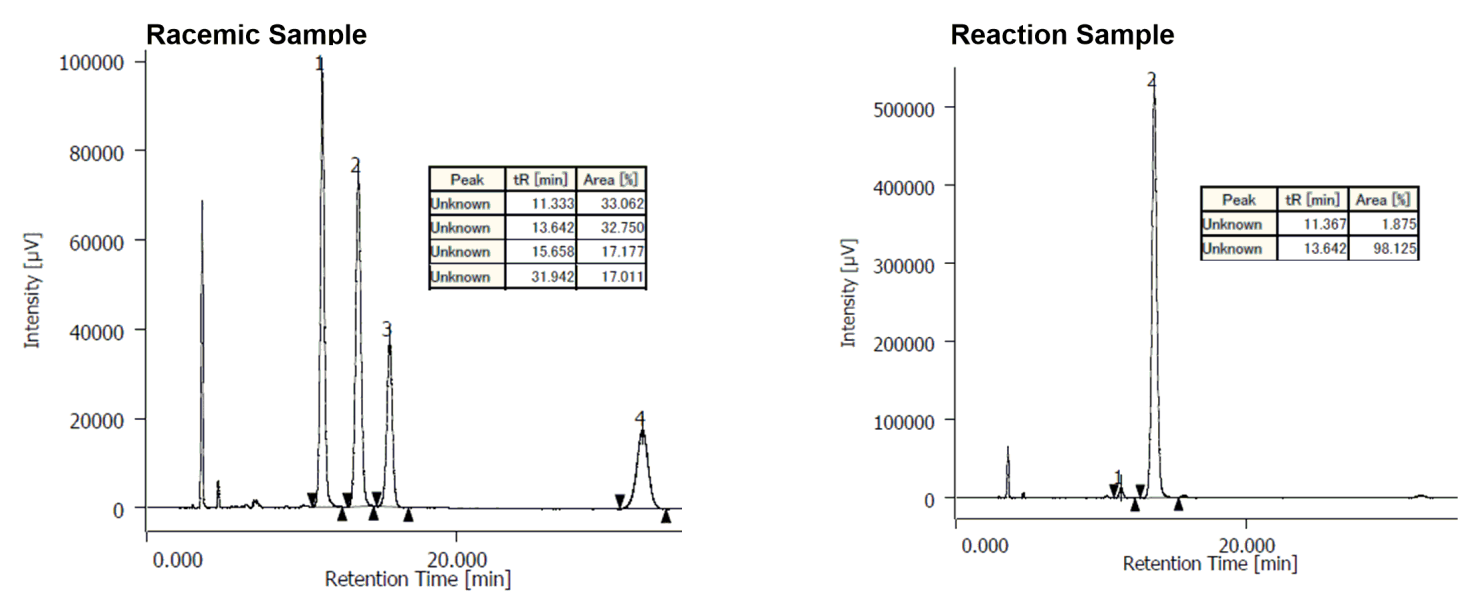

tert-Butyl ((1S,2S)-2-(2,3-dihydro-1H-pyrrolo[2,3-b]pyridine-1-carbonyl)-3,3,3-trifluoro-1-(3-methoxyphenyl)propyl)-

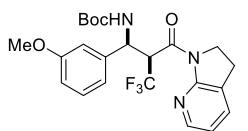
carbamate $(3 \mathrm{~g})$.

The reaction was performed according to procedure A using $N$-Boc imine $2 \mathrm{~g}$ (47.1 $\mathrm{mg})$ to afford 41.9 $\mathrm{mg}(90 \%)$ of the desired pure product $3 \mathrm{~g}$ after purification by flash column chromatography (hexane/ethyl acetate, 2:1).

White solid, m. p.: $147^{\circ} \mathrm{C}$; ${ }^{1} \mathrm{H}$ NMR (400 MHz, $\left.343 \mathrm{~K}, \mathrm{DMSO}-\mathrm{d}_{6}\right): \delta=8.16(\mathrm{~d}, J=5.0 \mathrm{~Hz}, 1 \mathrm{H}), 7.63(\mathrm{brs}, 1 \mathrm{H}), 7.58(\mathrm{~d}, J=$ $7.3 \mathrm{~Hz}, 1 \mathrm{H}), 7.04-6.99(\mathrm{~m}, 2 \mathrm{H}), 6.88(\mathrm{~s}, 1 \mathrm{H}), 6.77$ (d, J = 7.6 Hz, 1H), $6.66(\mathrm{dd}, J=8.1,2.4 \mathrm{~Hz}, 1 \mathrm{H}), 6.44-6.35$ (m, $1 \mathrm{H}), 5.24$ (brt, $J=9.4 \mathrm{~Hz}, 1 \mathrm{H}), 3.82-3.74(\mathrm{~m}, 1 \mathrm{H}), 3.51-3.44(\mathrm{~m}, 1 \mathrm{H}), 3.47(\mathrm{~s}, 3 \mathrm{H}), 2.86(\mathrm{ddd}, J=16.0,10.4,5.3 \mathrm{~Hz}, 1 \mathrm{H}), 2.60(\mathrm{ddd}, J=$ 16.6, 10.0, 7.3 Hz, 1H), 1.36 ppm (s, 9H); ${ }^{13} \mathrm{C}$ NMR (150 MHz, $\left.343 \mathrm{~K}, \mathrm{DMSO}-d_{6}\right): \delta=163.3$ (q, J = 2.9 Hz), $158.7,154.1$ (br), $153.9,145.1,140.6,134.0,128.4,126.2,124.8(q, J=282.3 \mathrm{~Hz}), 119.6,118.8,113.4,112.1,77.9,54.5,52.9$ (br), 50.7 (q, $J=22.2$ Hz), 45.5, 27.9, 23.0 ppm; ${ }^{19} \mathrm{~F}$ NMR (376 MHz, $\left.343 \mathrm{~K}, \mathrm{DMSO}-d_{6}\right): \delta=-61.6$ ppm (m); IR (film): $\widetilde{v}=3351,3007,2974,2935$, 1704, 1663, 1594, 1490, 1425, 1366, 1312, 1265, 1153, 1124, 1101, 1042, $865 \mathrm{~cm}^{-1}$; HRMS (ESI): m/z calculated for $\mathrm{C}_{23} \mathrm{H}_{26} \mathrm{~F}_{3} \mathrm{~N}_{3} \mathrm{O}_{4} \mathrm{Na}[\mathrm{M}+\mathrm{Na}]^{+}: 488.1768$, found: $488.1762 ;[\alpha]_{D}^{24}=-58.7\left(c=1.45, \mathrm{CHCl}_{3}, 96 \%\right.$ ee sample); HPLC analysis $($ CHIRALPAK IA $(\phi=0.46 \mathrm{~cm} \times 25 \mathrm{~cm}), 2$-propanol $/ n$-hexane $=1 / 9$, flow rate $=1.0 \mathrm{~mL} / \mathrm{min}$, detection at $254 \mathrm{~nm}$, tR $=$ $18.8 \mathrm{~min}$ (major), $22.9 \mathrm{~min}$ (minor)): 

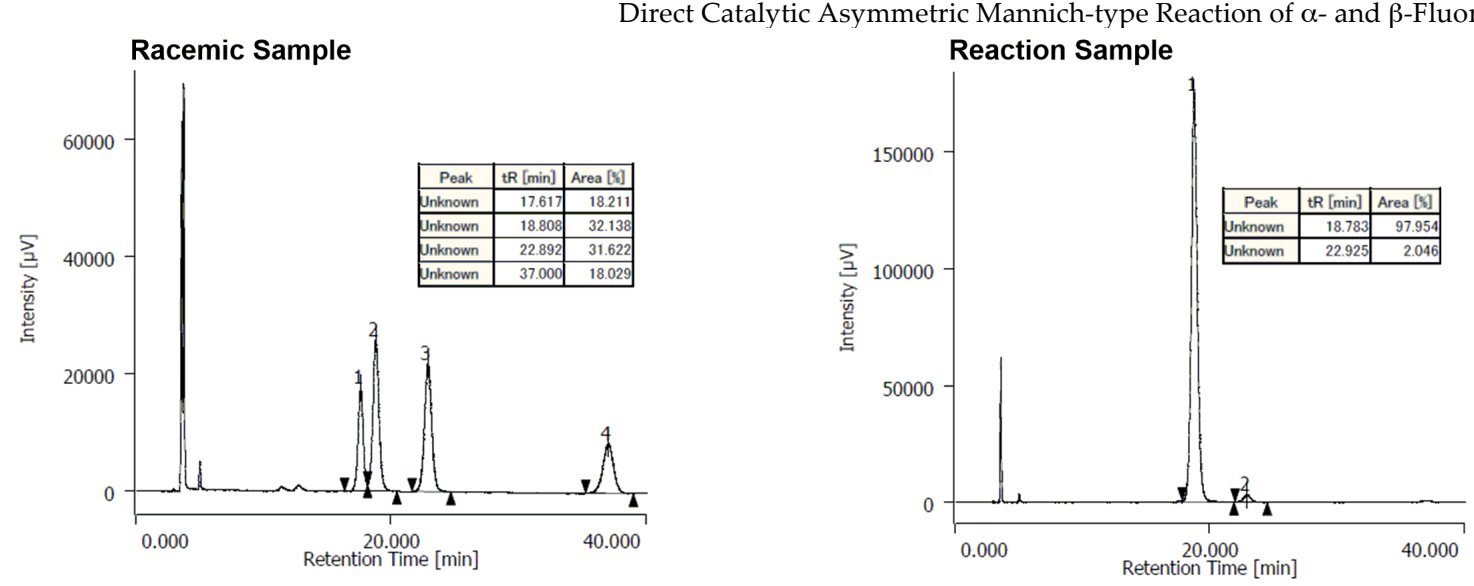

tert-Butyl ((1S,2S)-2-(2,3-dihydro-1H-pyrrolo[2,3-b]pyridine-1-carbonyl)-3,3,3-trifluoro-1-(4-methoxyphenyl)propyl)-

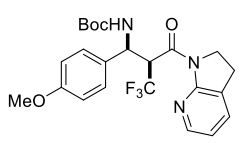
carbamate (3h).

A flame-dried $100 \mathrm{~mL}$ test tube equipped with a magnetic stirring bar and a 3-way glass stopcock was charged with $\left[\mathrm{Cu}\left(\mathrm{CH}_{3} \mathrm{CN}\right)_{4}\right] \mathrm{PF}_{6} \quad(121.1 \quad \mathrm{mg}, \quad 0.33 \quad \mathrm{mmol}, \quad 5 \quad \mathrm{~mol} \%)$ and (R)-(-)-2,2'-bis[di(3,5-diisopropyl-4-dimethylaminophenyl)phosphine]-6,6'-dimethoxy1,1'-biphenyl

(DIPA-MeO-Biphep) (425.7 mg, $0.39 \mathrm{mmol}, 6 \mathrm{~mol} \%$ ) in a glove box under Ar atmosphere. Afterwards, the test tube was removed from the glove box and evacuated for $15 \mathrm{~min}$. Then, it was backfilled with Ar and anhydrous THF (13 mL, 0.5 M) was added at ambient temperature. The resulting clear colorless solution was stirred for $1 \mathrm{~h}$ before amide $1 \mathrm{a}$ (1.5 $\mathrm{g}$, $6.5 \mathrm{mmol}, 1.0$ equiv.), $\mathrm{N}$-Boc imine $2 \mathrm{~h}(3.1 \mathrm{~g}, 13.0 \mathrm{mmol}, 2.0$ equiv.) and Barton's base ( $38.2 \mu \mathrm{L}, 0.33 \mathrm{mmol}, 5 \mathrm{~mol} \%)$ were subsequently added. The resulting red solution was allowed to stir for $24 \mathrm{~h}$ before it was diluted with water and extracted three times with ethyl acetate. The combined organic extracts were dried over $\mathrm{Na}_{2} \mathrm{SO}_{4}$, filtered and evaporated to afford $2.85 \mathrm{~g}$ (94\%) of the desired pure product $3 \mathrm{~h}$ after purification by flash column chromatography (hexane/ethyl acetate, 3:1).

White solid, m. p.: $157-158^{\circ} \mathrm{C}$; ${ }^{1} \mathrm{H}$ NMR $\left(600 \mathrm{MHz}, 343 \mathrm{~K}\right.$, DMSO- $\left.d_{6}\right): \delta=8.15$ (d, J= 5.2 Hz, 1H), 7.60 (brs, $\left.1 \mathrm{H}\right), 7.57$ (d, $J$ $=7.2 \mathrm{~Hz}, 1 \mathrm{H}), 7.15(\mathrm{~d}, J=8.2 \mathrm{~Hz}, 2 \mathrm{H}), 7.03(\mathrm{dd}, J=6.7,5.7 \mathrm{~Hz}, 1 \mathrm{H}), 6.62(\mathrm{~d}, J=8.2 \mathrm{~Hz}, 2 \mathrm{H}), 6.38-6.32(\mathrm{~m}, 1 \mathrm{H}), 5.23-5.20$ (brm, 1H), 3.80-3.75 (m, 1H), $3.63(\mathrm{~s}, 3 \mathrm{H}), 3.54-3.49(\mathrm{~m}, 1 \mathrm{H}), 2.88-2.83(\mathrm{~m}, 1 \mathrm{H}), 2.63-2.57(\mathrm{~m}, 1 \mathrm{H}), 1.35 \mathrm{ppm}(\mathrm{s}, 9 \mathrm{H}) ;{ }^{13} \mathrm{C}$ NMR (150 MHz, 343 K, DMSO-d6): $\delta=163.4$ (br), 158.3, 154.1 (br), 154.0, 145.0, 134.0, 131.3, 128.3, 126.2, 124.8 (q, $J=$ $281.8 \mathrm{~Hz}), 118.8,112.8,77.8,54.7,52.3(\mathrm{br}), 50.9(\mathrm{q}, J=23.6 \mathrm{~Hz}), 45.5,27.9,23.0$ ppm; ${ }^{19} \mathrm{~F}$ NMR $(376 \mathrm{MHz}, 343 \mathrm{~K}$, DMSO- $\left.d_{6}\right): \delta=-61.6$ ppm (m); IR (film): $\widetilde{v}=3348,3062,3004,2975,2935,1705,1662,1612,1594,1514,1476,1443,1425$, $1366,1248,1152,1124,1111,1033,898,866,833,786,756 \mathrm{~cm}^{-1}$; HRMS (ESI): $m / z$ calculated for $\mathrm{C}_{23} \mathrm{H}_{26} \mathrm{~F}_{3} \mathrm{~N}_{3} \mathrm{O}_{4} \mathrm{Na}[\mathrm{M}+\mathrm{Na}]^{+}$: 488.1768, found: 488.1755; $[\alpha]_{D}^{24}=-85.2\left(c=1.40, \mathrm{CHCl}_{3}, 98 \%\right.$ ee sample); HPLC analysis (CHIRALPAK IE $(\phi=0.46 \mathrm{~cm}$ x $25 \mathrm{~cm}$ ), 2-propanol $/ n$-hexane $=1 / 10$, flow rate $=1.0 \mathrm{~mL} / \mathrm{min}$, detection at $254 \mathrm{~nm}, \mathrm{t}_{\mathrm{R}}=29.6 \mathrm{~min}$ (major), $46.1 \mathrm{~min}$ (minor)): 

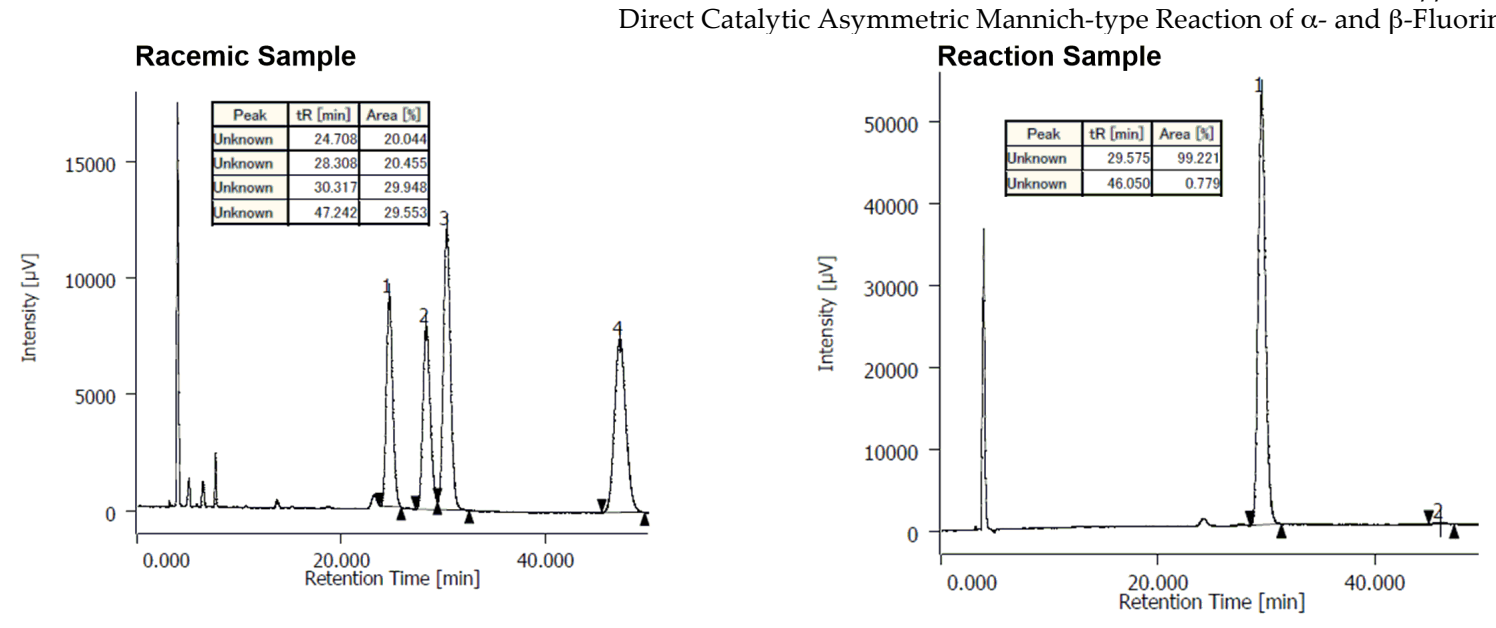

tert-Butyl ((1S,2S)-2-(2,3-dihydro-1H-pyrrolo[2,3-b]pyridine-1-carbonyl)-3,3,3-trifluoro-1-(2-fluorophenyl)propyl)-

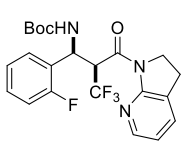
carbamate (3i).

The reaction was performed according to procedure A using $N$-Boc imine $2 \mathbf{i}(44.7 \mathrm{mg})$ to afford $43.3 \mathrm{mg}$ $(95 \%)$ of the desired product $3 \mathbf{i}$ as a $2.8: 1$ mixture of diastereomers after purification by flash column chromatography (hexane/ethyl acetate, 2:1) in form of a white foam. Analytical data for the major diastereomer are listed below:

White solid, m. p.: $148-150{ }^{\circ} \mathrm{C} ;{ }^{1} \mathrm{H}$ NMR (400 MHz, $\left.343 \mathrm{~K}, \mathrm{DMSO}-\mathrm{d}_{6}\right): \delta=8.18-8.16$ (m, 1H), 7.60 (ddd, J = 7.4, $2.7,1.3 \mathrm{~Hz}$, 1H), 7.44 (brs, 1H), 7.38 (td, $J=11.5,1.6 \mathrm{~Hz}, 1 \mathrm{H}), 7.20-7.14(\mathrm{~m}, 1 \mathrm{H}), 7.05(\mathrm{dd}, J=7.4,5.1 \mathrm{~Hz}, 1 \mathrm{H}), 7.03-6.98(\mathrm{~m}, 1 \mathrm{H}), 6.82$ $(\mathrm{td}, J=7.6,1.0 \mathrm{~Hz}, 1 \mathrm{H}), 6.54-6.45(\mathrm{~m}, 1 \mathrm{H}), 5.70(\mathrm{t}, J=10.3 \mathrm{~Hz}, 1 \mathrm{H}), 3.81(\mathrm{ddd}, J=12.1,10.5,7.2 \mathrm{~Hz}, 1 \mathrm{H}), 3.47(\mathrm{ddd}, J=$ $12.1,10.6,5.3 \mathrm{~Hz}, 1 \mathrm{H}), 2.93-2.85(\mathrm{~m}, 1 \mathrm{H}), 2.70-2.61(\mathrm{~m}, 1 \mathrm{H}), 1.36 \mathrm{ppm}(\mathrm{s}, 9 \mathrm{H}) ;{ }^{13} \mathrm{C}$ NMR $(100 \mathrm{MHz}, 343 \mathrm{~K}, \mathrm{DMSO}-d 6): \delta=$ $162.7(\mathrm{q}, J=2.9 \mathrm{~Hz}), 158.7(\mathrm{~d}, J=247.2 \mathrm{~Hz}), 153.9(\mathrm{br}), 153.8,145.0,134.0,129.1(\mathrm{~d}, J=8.2 \mathrm{~Hz}), 128.9(\mathrm{brd}, J=3.1 \mathrm{~Hz})$, $126.2(\mathrm{~d}, J=22.7 \mathrm{~Hz}), 126.1,124.7(\mathrm{q}, J=285.4 \mathrm{~Hz}), 123.2(\mathrm{~d}, J=3.5 \mathrm{~Hz}), 118.8,114.6(\mathrm{~d}, J=22.6 \mathrm{~Hz}), 78.0,50.1(\mathrm{q}, J=24.0$ Hz), 45.7 (br), 45.5, 27.7, 22.9 ppm; ${ }^{19} \mathrm{~F}$ NMR (376 MHz, $\left.343 \mathrm{~K}, \mathrm{DMSO}-d_{6}\right): \delta=-62.1$ (s, 3F), -116.6 ppm (s, 1F); IR (film): $\widetilde{v}=3355$ (br), 2978, 2928, 1708, 1665, 1594, 1493, 1426, 1366, 1313, 1268, 1155, 1103, 1022,760 cm-1; HRMS (ESI): $m / z$ calculated for $\mathrm{C}_{22} \mathrm{H}_{24} \mathrm{~F}_{4} \mathrm{~N}_{3} \mathrm{O}_{3}[\mathrm{M}+\mathrm{H}]^{+}: 454.1748$, found: $454.1744 ;[\alpha]_{D}^{25}=-66.9\left(c=0.60, \mathrm{CHCl}_{3}, 74 \%\right.$ ee sample); HPLC analysis (CHIRALPAK IA $(\phi=0.46 \mathrm{~cm} \times 25 \mathrm{~cm}), 2$-propanol $/ n$-hexane $=1 / 9$, flow rate $=1.0 \mathrm{~mL} / \mathrm{min}$, detection at $254 \mathrm{~nm}, \mathrm{tr}_{\mathrm{R}}=13.5 \mathrm{~min}$ (minor), $18.1 \mathrm{~min}($ major $)$ ):
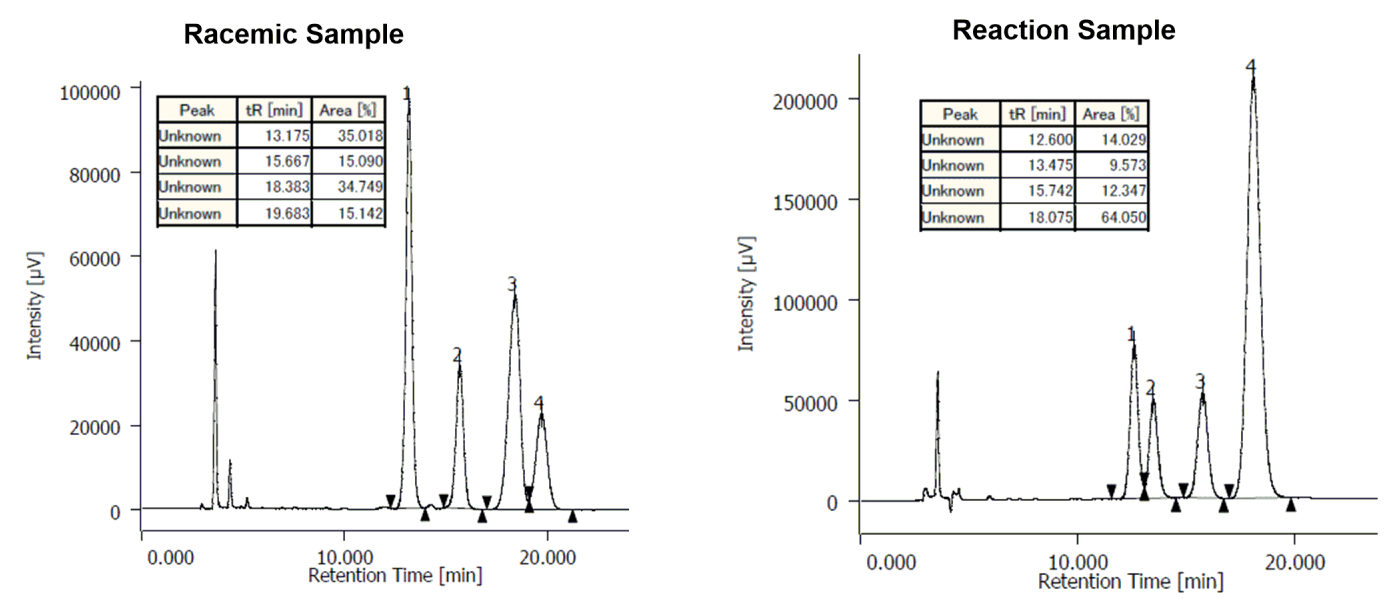

tert-Butyl ((1S,2S)-2-(2,3-dihydro-1H-pyrrolo[2,3-b]pyridine-1-carbonyl)-3,3,3-trifluoro-1-(3-fluorophenyl)propyl)-

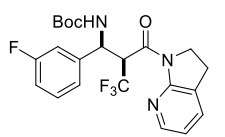
carbamate $(3 \mathbf{j})$.

The reaction was performed according to procedure A using N-Boc imine $\mathbf{2} \mathbf{j}(44.6 \mathrm{mg}$ ) to afford $39.0 \mathrm{mg}$ $(86 \%)$ of the desired pure product $3 \mathbf{j}$ after purification by flash column chromatography (hexane/ethyl acetate, 2:1).

White solid, m. p.: $169-171^{\circ} \mathrm{C}$; ${ }^{1} \mathrm{H}$ NMR (400 MHz, $343 \mathrm{~K}$, DMSO-d 6 ): $\delta=8.17-8.15$ (m, $\left.1 \mathrm{H}\right), 7.64$ (brs, $\left.1 \mathrm{H}\right), 7.60$ (ddd, $J=$ 7.4, 2.7, $1.3 \mathrm{~Hz}, 1 \mathrm{H}), 7.16(\mathrm{td}, J=11.8,6.1 \mathrm{~Hz}, 1 \mathrm{H}), 7.10(\mathrm{ddd}, J=10.4,2.2,1.8 \mathrm{~Hz}, 1 \mathrm{H}), 7.07-7.03(\mathrm{~m}, 2 \mathrm{H}), 6.94-6.89(\mathrm{~m}$, 1H), 6.45-6.36 (m, 1H), $5.28(\mathrm{t}, J=10.2 \mathrm{~Hz}, 1 \mathrm{H}), 3.82(\mathrm{ddd}, J=12.1,10.5,7.1 \mathrm{~Hz}, 1 \mathrm{H}), 3.52(\mathrm{ddd}, J=12.1,10.6,5.4 \mathrm{~Hz}, 1 \mathrm{H})$, 2.94-2.85 (m, 1H), 2.70-2.61 (m, 1H), 1.36 ppm (s, 9H); ${ }^{13} \mathrm{C}$ NMR (150 MHz, $\left.343 \mathrm{~K}, \mathrm{DMSO}-d_{6}\right): \delta=163.0(\mathrm{br}), 161.4(\mathrm{~d}, J=$ $243.8 \mathrm{~Hz}), 154.1(\mathrm{br}), 153.8,145.0,141.8(\mathrm{~d}, J=6.8 \mathrm{~Hz}), 134.2,129.4(\mathrm{~d}, J=8.1 \mathrm{~Hz}), 126.1,124.6(\mathrm{q}, J=281.8 \mathrm{~Hz}), 123.3$, 118.9, $113.9(\mathrm{~d}, J=21.0 \mathrm{~Hz}), 113.8(\mathrm{~d}, J=22.2 \mathrm{~Hz}), 78.1,52.5(\mathrm{br}), 50.6(\mathrm{q}, J=24.0 \mathrm{~Hz}), 45.5,27.8,23.0$ ppm; ${ }^{19} \mathrm{~F}$ NMR $(376$ MHz, 343 K, DMSO- $\left.d_{6}\right): \delta=-61.8$ (s, 3F), -113.3 ppm (s, 1F); IR (film): $\widetilde{v}=3341$ (br), 3062, 2976, 2928, 1705, 1662, 1594, 1426, 1367, 1311, 1257, 1220, 1154, 868, $772 \mathrm{~cm}^{-1}$; HRMS (ESI): $\mathrm{m} / z$ calculated for $\mathrm{C}_{22} \mathrm{H}_{23} \mathrm{~F}_{4} \mathrm{~N}_{3} \mathrm{O} \mathrm{O}_{3} \mathrm{Na}[\mathrm{M}+\mathrm{Na}]^{+}: 476.1568$, found: 476.1562; $[\alpha]_{D}^{25}=-31.4\left(c=0.15, \mathrm{CHCl}_{3}, 95 \%\right.$ ee sample); HPLC analysis (CHIRALPAK IC $(\phi=0.46 \mathrm{~cm} \times 25 \mathrm{~cm})$, 2-propanol $/ n$-hexane $=1 / 9$, flow rate $=1.0 \mathrm{~mL} / \mathrm{min}$, detection at $254 \mathrm{~nm}, \mathrm{tR}=26.2 \mathrm{~min}$ (major), $51.5 \mathrm{~min}(\operatorname{minor})$ ):
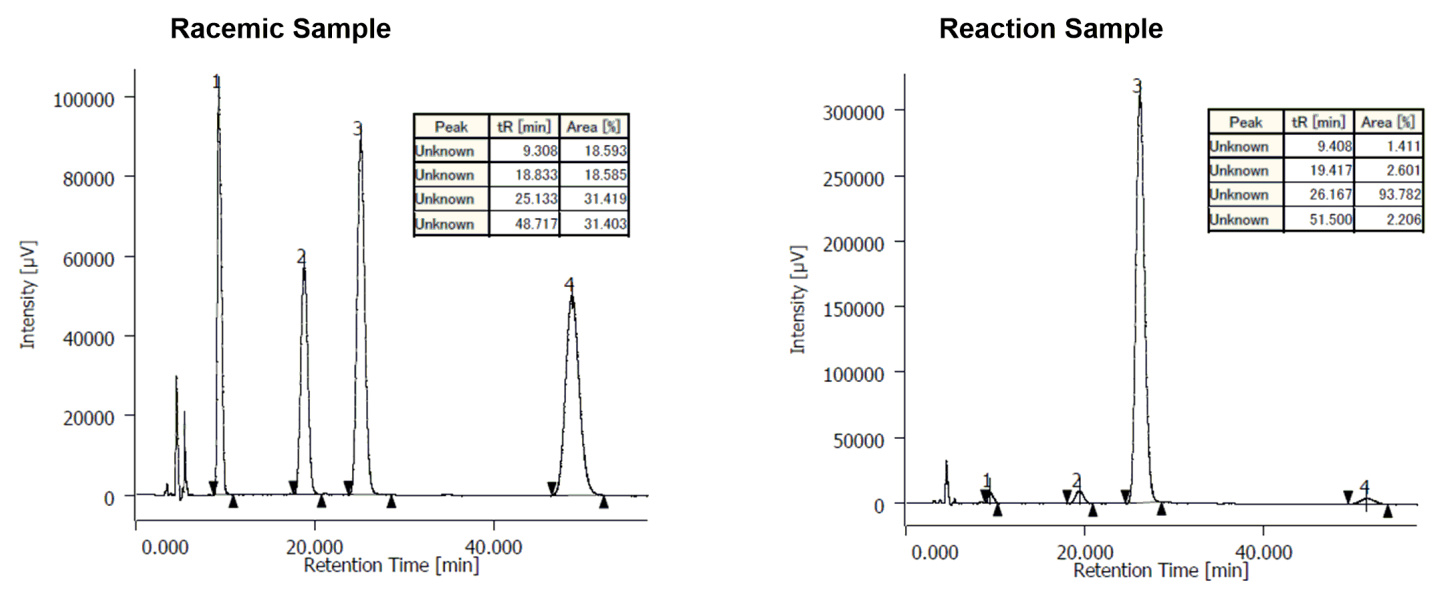

tert-Butyl ((1S,2S)-2-(2,3-dihydro-1H-pyrrolo[2,3-b]pyridine-1-carbonyl)-3,3,3-trifluoro-1-(4-fluorophenyl)propyl)-

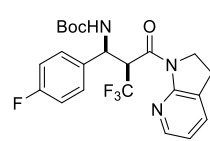
carbamate (3k).

The reaction was performed according to procedure A using N-Boc imine $\mathbf{2 k}(44.6 \mathrm{mg}$ ) to afford 41.9 $\mathrm{mg}(92 \%)$ of the desired pure product $3 \mathbf{k}$ after purification by flash column chromatography (hexane/ethyl acetate, 2:1).

White solid, m. p.: $192{ }^{\circ} \mathrm{C} ;{ }^{1} \mathrm{H}$ NMR $\left(400 \mathrm{MHz}, 343 \mathrm{~K}, \mathrm{DMSO}-\mathrm{d}_{6}\right): \delta=8.15$ (dd, J= 5.0, $\left.1.1 \mathrm{~Hz}, 1 \mathrm{H}\right), 7.70(\mathrm{brs}, 1 \mathrm{H}), 7.59$ (dd, $J=7.4,1.3 \mathrm{~Hz}, 1 \mathrm{H}), 7.32-7.26(\mathrm{~m}, 2 \mathrm{H}), 7.04(\mathrm{dd}, J=7.3,5.0 \mathrm{~Hz}, 1 \mathrm{H}), 6.92-6.86(\mathrm{~m}, 2 \mathrm{H}), 6.44-6.35(\mathrm{~m}, 1 \mathrm{H}), 5.27$ (brt, $J=9.6$ $\mathrm{Hz}, 1 \mathrm{H}), 3.81(\mathrm{ddd}, J=12.1,10.6,7.3 \mathrm{~Hz}, 1 \mathrm{H}), 3.52(\mathrm{ddd}, J=11.9,10.5,5.3 \mathrm{~Hz}, 1 \mathrm{H}), 2.88(\mathrm{ddd}, J=16.6,10.5,5.2 \mathrm{~Hz}, 1 \mathrm{H})$, $2.65(\mathrm{ddd}, J=16.9,10.5,7.1 \mathrm{~Hz}, 1 \mathrm{H}), 1.35 \mathrm{ppm}(\mathrm{s}, 9 \mathrm{H}) ;{ }^{13} \mathrm{C}$ NMR $\left(150 \mathrm{MHz}, 343 \mathrm{~K}, \mathrm{DMSO}-d_{6}\right): \delta=163.2(\mathrm{q}, J=3.4 \mathrm{~Hz})$, $161.1(\mathrm{~d}, J=244.2 \mathrm{~Hz}), 154.1$ (br), 153.8, 145.0, 135.4 (br), 134.1, 129.2 (d, J = 8.7 Hz), 126.2, 124.7 (q, J = 281.8 Hz), 118.9, $114.1(\mathrm{~d}, J=21.7 \mathrm{~Hz}), 78.0,52.2(\mathrm{br}), 50.8(\mathrm{q}, J=23.1 \mathrm{~Hz}), 45.5,27.8,23.0$ ppm; ${ }^{19} \mathrm{~F}$ NMR $\left(376 \mathrm{MHz}, 343 \mathrm{~K}, \mathrm{DMSO}-d_{6}\right): \delta=$ -61.6 (s, 3F), -114.7 ppm (s, 1F); IR (film): $\widetilde{v}=3333,3069,3007,2978,2932,1706,1662,1594,1510,1477,1444,1426,1367$, 

$1313,1257,1241,1154,1125,1100,1045,1022,967,898,838 \mathrm{~cm}^{-1}$; HRMS (ESI): $m / z$ calculated for $\mathrm{C}_{22} \mathrm{H}_{23} \mathrm{~F}_{4} \mathrm{~N}_{3} \mathrm{O}_{3} \mathrm{Na}$ $[\mathrm{M}+\mathrm{Na}]^{+}:$476.1568, found: 476.1563; $[\alpha]_{D}^{24}=-66.0\left(c=1.55, \mathrm{CHCl}_{3}, 99 \%\right.$ ee sample); HPLC analysis (CHIRALPAK IA $(\phi=0.46 \mathrm{~cm} \times 25 \mathrm{~cm}), 2$-propanol $/ n$-hexane $=1 / 9$, flow rate $=1.0 \mathrm{~mL} / \mathrm{min}$, detection at $254 \mathrm{~nm}, \mathrm{tR}=11.2 \mathrm{~min}(\mathrm{minor})$, $12.8 \min ($ major)):
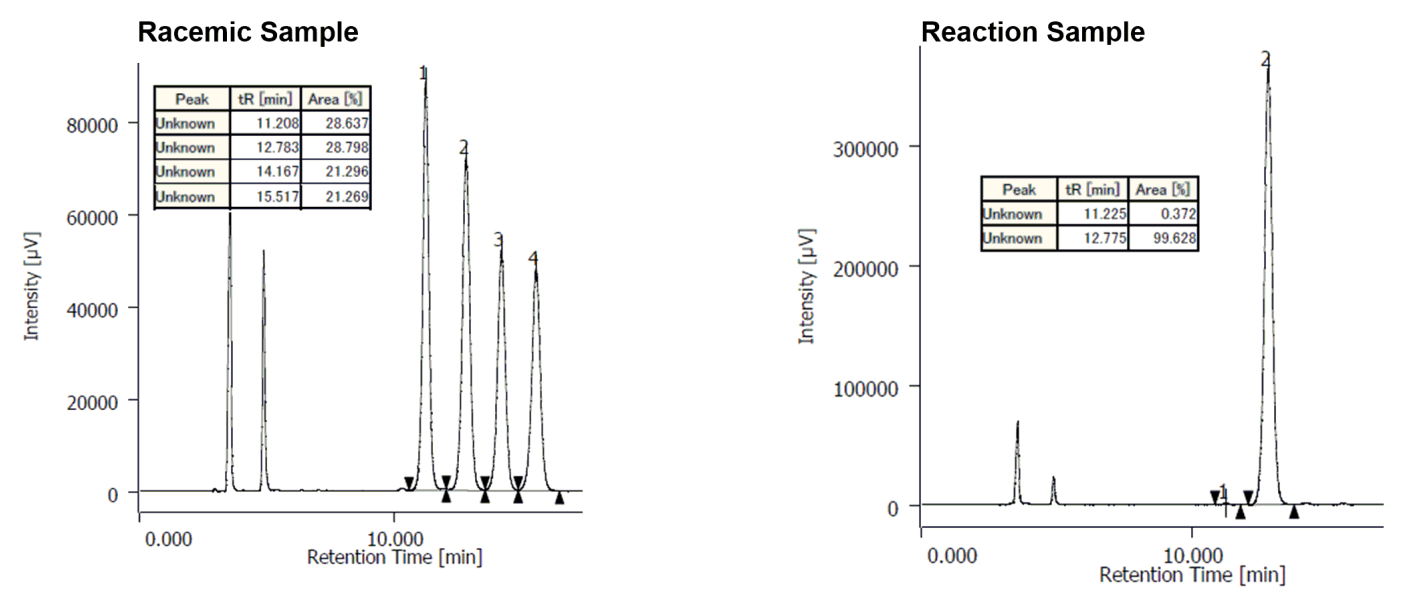

tert-Butyl ((1S,2S)-2-(2,3-dihydro-1H-pyrrolo[2,3-b]pyridine-1-carbonyl)-3,3,3-trifluoro-1-(4-chlorophenyl)propyl)-

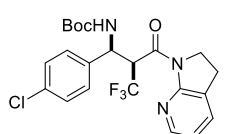
carbamate (31).

The reaction was performed according to procedure A using N-Boc imine 21 (47.9 $\mathrm{mg})$ to afford 43.0 mg $(91 \%)$ of the desired pure product 31 after purification by flash column chromatography (hexane/ethyl acetate, 3:1).

White solid, m. p.: $202{ }^{\circ} \mathrm{C}$; ${ }^{1} \mathrm{H}$ NMR $\left(400 \mathrm{MHz}, 343 \mathrm{~K}, \mathrm{DMSO}-\mathrm{d}_{6}\right): \delta=8.15$ (d, J=4.6 Hz, $\left.1 \mathrm{H}\right), 7.72(\mathrm{brs}, 1 \mathrm{H}), 7.61(\mathrm{~d}, J=$ $7.3 \mathrm{~Hz}, 1 \mathrm{H}), 7.27(\mathrm{~d}, J=6.9 \mathrm{~Hz}, 2 \mathrm{H}), 7.15-7.12(\mathrm{~m}, 2 \mathrm{H}), 7.07-7.03(\mathrm{~m}, 1 \mathrm{H}), 6.43-6.35(\mathrm{~m}, 1 \mathrm{H}), 5.25(\mathrm{t}, J=9.8 \mathrm{~Hz}, 1 \mathrm{H})$, 3.85-3.77 (m, 1H), 3.56-3.50 (m, 1H), 2.89 (ddd, $J=15.7,10.4,5.4 \mathrm{~Hz}, 1 \mathrm{H}), 2.69-2.61(\mathrm{~m}, 1 \mathrm{H}), 1.36 \mathrm{ppm}(\mathrm{s}, 9 \mathrm{H}) ;{ }^{13} \mathrm{C} \mathrm{NMR}$ $\left(150 \mathrm{MHz}, 343 \mathrm{~K}, \mathrm{DMSO}-d_{6}\right): \delta=163.1$ (q, $\left.J=2.9 \mathrm{~Hz}\right), 154.1$ (br), 153.8, 145.0, 138.1, 134.1, 131.9, 128.9, $127.4,126.2,124.6$ $(\mathrm{q}, J=282.3 \mathrm{~Hz}), 118.9,78.1,52.3(\mathrm{br}), 50.6(\mathrm{q}, J=24.1 \mathrm{~Hz}), 45.5,27.8,23.0$ ppm; ${ }^{19} \mathrm{~F}$ NMR $\left(376 \mathrm{MHz}, 343 \mathrm{~K}, \mathrm{DMSO}-d_{6}\right): \delta=$ -61.6 ppm (s); IR (film): $\widetilde{v}=3337,2973,2928,1706,1663,1594,1493,1426,1367,1263,1154,786,758$ cm-1; HRMS (ESI): $m / z$ calculated for $\mathrm{C}_{22} \mathrm{H}_{23} \mathrm{ClF}_{3} \mathrm{~N}_{3} \mathrm{O}_{3} \mathrm{Na}[\mathrm{M}+\mathrm{Na}]^{+}: 492.1272$, found: $492.1266 ;[\alpha]_{D}^{24}=-72.1\left(c=0.85, \mathrm{CHCl}_{3}, 99 \%\right.$ ee sample); HPLC analysis (CHIRALPAK IA $(\phi=0.46 \mathrm{~cm} \times 25 \mathrm{~cm})$, 2-propanol $/ n$-hexane $=1 / 9$, flow rate $=1.0 \mathrm{~mL} / \mathrm{min}$, detection at $254 \mathrm{~nm}, \mathrm{t}=12.5 \min ($ minor), $14.2 \min ($ major $)$ ):
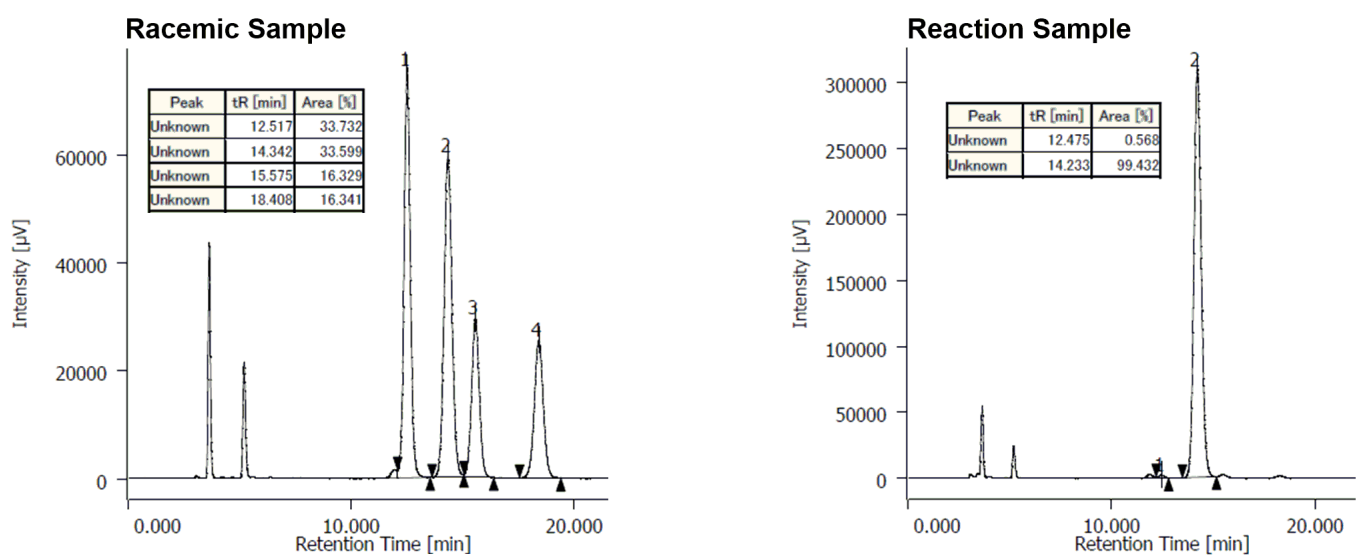

tert-Butyl ((1S,2S)-2-(2,3-dihydro-1H-pyrrolo[2,3-b]pyridine-1-carbonyl)-3,3,3-trifluoro-1-(3-nitrophenyl)propyl)-

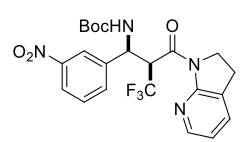

\section{carbamate $(3 \mathrm{~m})$.}

The reaction was performed according to procedure $\mathrm{A}$ at a reaction temperature of $-60{ }^{\circ} \mathrm{C}$ using $\mathrm{N}$-Boc imine $2 \mathrm{~m}(50.1 \mathrm{mg})$ to afford $44.0 \mathrm{mg}(92 \%)$ of the desired product $3 \mathrm{~m}$ as a $5.0: 1$ mixture of diastereomers after purification by flash column chromatography (hexane/ethyl acetate, 2:1). Analytical data for the major diastereomer are listed below:

White solid, m. p.: $148-151{ }^{\circ} \mathrm{C} ;{ }^{1} \mathrm{H}$ NMR $\left(400 \mathrm{MHz}, 343 \mathrm{~K}, \mathrm{DMSO}-d_{6}\right): \delta=8.23(\mathrm{t}, J=2.0 \mathrm{~Hz}, 1 \mathrm{H}), 8.16-8.14(\mathrm{~m}, 1 \mathrm{H}), 7.96$ $(\mathrm{ddd}, J=8.2,2.3,0.9 \mathrm{~Hz}, 1 \mathrm{H}), 7.91(\mathrm{brs}, 1 \mathrm{H}), 7.67(\mathrm{dt}, J=7.6,1.2 \mathrm{~Hz}, 1 \mathrm{H}), 7.56(\mathrm{ddd}, J=7.4,2.6,1.2 \mathrm{~Hz}, 1 \mathrm{H}), 7.46(\mathrm{t}, J=7.9$ $\mathrm{Hz}, 1 \mathrm{H}), 7.02(\mathrm{dd}, J=7.4,5.1 \mathrm{~Hz}, 1 \mathrm{H}), 6.55-6.47(\mathrm{~m}, 1 \mathrm{H}), 5.37$ (brt, $J=10.2 \mathrm{~Hz}, 1 \mathrm{H}), 3.81$ (ddd, J= 12.2, 10.5, 6.9 Hz, 1H), $3.50(\mathrm{ddd}, J=12.2,10.6,5.5 \mathrm{~Hz}, 1 \mathrm{H}), 2.92-2.84(\mathrm{~m}, 1 \mathrm{H}), 2.65-2.57(\mathrm{~m}, 1 \mathrm{H}), 1.36 \mathrm{ppm}(\mathrm{s}, 9 \mathrm{H}) ;{ }^{13} \mathrm{C}$ NMR $(125 \mathrm{MHz}, 343 \mathrm{~K}$, DMSO-d6): $\delta=162.9,154.2$ (br), 153.5, 147.2, 145.1, 141.1, 134.2, 133.8, 129.1, 126.1, 124.5 (q, $J=282.0 \mathrm{~Hz}$ ), 122.1, 122.0, 119.0, 78.3, 52.5 (br), 50.3 (q, $J=23.8 \mathrm{~Hz}), 45.4,27.8,22.9$ ppm; ${ }^{19} \mathrm{~F}$ NMR (376 MHz, $\left.343 \mathrm{~K}, \mathrm{DMSO}-d_{6}\right): \delta=-61.8 \mathrm{ppm}(\mathrm{s}) ;$ IR (film): $\widetilde{v}=3354$ (br), 3073, 2963, 2921, 2849, 1709, 1594, 1531, 1426, 1352, 1256, 1156, $771 \mathrm{~cm}^{-1}$; HRMS (ESI): m/z calculated for $\mathrm{C}_{22} \mathrm{H}_{24} \mathrm{~F}_{3} \mathrm{~N}_{4} \mathrm{O}_{5}[\mathrm{M}+\mathrm{H}]^{+}: 481.1693$, found: 481.1692; $[\alpha]_{D}^{26}=-11.5$ (c=0.02, $\mathrm{CHCl}_{3}, 89 \%$ ee sample); HPLC analysis (CHIRALPAK IC $(\phi=0.46 \mathrm{~cm} \times 25 \mathrm{~cm}), 2$-propanol $/ n$-hexane $=1 / 9$, flow rate $=1.0 \mathrm{~mL} / \mathrm{min}$, detection at $254 \mathrm{~nm}$, $\mathrm{t}_{\mathrm{R}}=50.6 \mathrm{~min}$ (minor), $60.6 \mathrm{~min}$ (major)):
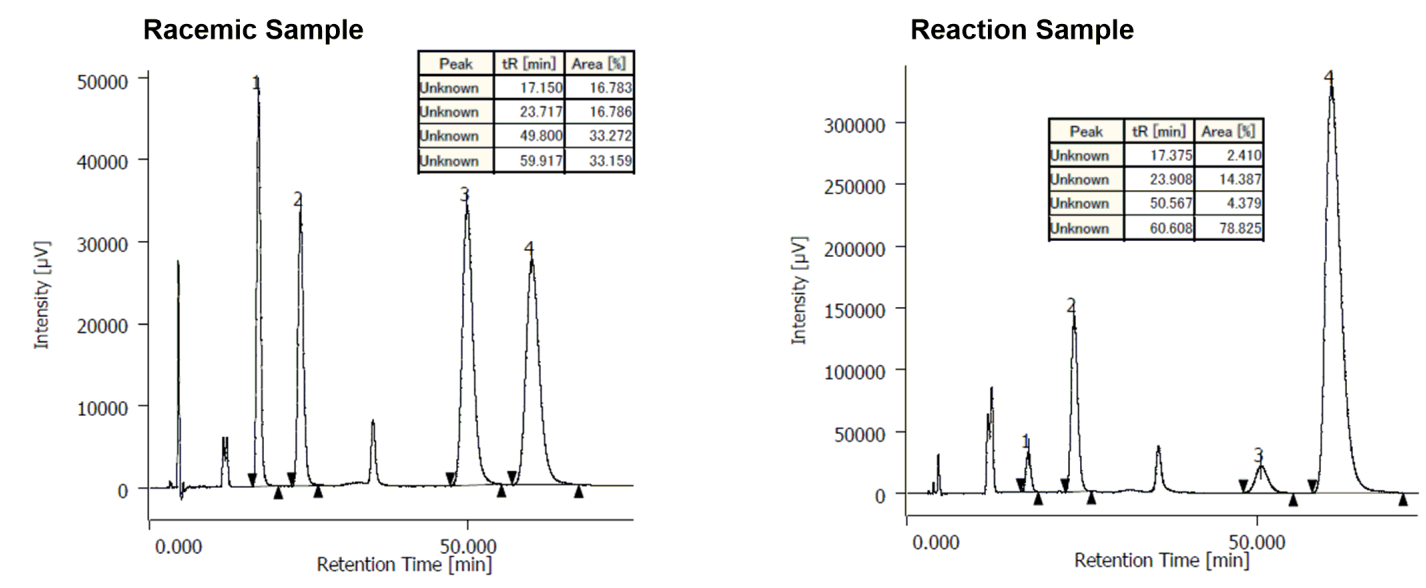

tert-Butyl ((1S,2S)-2-(2,3-dihydro-1H-pyrrolo[2,3-b]pyridine-1-carbonyl)-3,3,3-trifluoro-1-(4-((trifluoromethyl)-

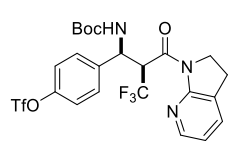
sulfonyl)phenyl)propyl)carbamate (3n).

The reaction was performed according to procedure A using N-Boc imine $2 \mathrm{n}(70.7 \mathrm{mg})$ to afford $52 \mathrm{mg}$ $(89 \%)$ of the desired product $3 \mathbf{n}$ as a 5.8:1 mixture of diastereomers after purification by flash column chromatography (hexane/ethyl acetate, 3:1). The major diastereomer was obtained in analytically pure form after flash column chromatography (hexane/ethyl acetate, 5:1 to 4:1), its analytical data are listed below:

White solid, m. p.: $97-99{ }^{\circ} \mathrm{C}$; ${ }^{1} \mathrm{H}$ NMR $\left(400 \mathrm{MHz}, 343 \mathrm{~K}, \mathrm{DMSO}-d_{6}\right): \delta=8.15(\mathrm{~d}, J=4.4 \mathrm{~Hz}, 1 \mathrm{H}), 7.82(\mathrm{brs}, 1 \mathrm{H}), 7.58(\mathrm{~d}, J=$ $7.3 \mathrm{~Hz}, 1 \mathrm{H}), 7.44(\mathrm{~d}, J=8.7 \mathrm{~Hz}, 2 \mathrm{H}), 7.20(\mathrm{~d}, J=8.7 \mathrm{~Hz}, 2 \mathrm{H}), 7.04(\mathrm{dd}, J=7.4,5.2 \mathrm{~Hz}, 1 \mathrm{H}), 6.47-6.38$ (m, 1H), 5.31 (brt, $J=$ $8.5 \mathrm{~Hz}, 1 \mathrm{H}), 3.81(\mathrm{ddd}, J=11.9,10.7,7.1 \mathrm{~Hz}, 1 \mathrm{H}), 3.47(\mathrm{ddd}, J=11.9,11.0,5.3 \mathrm{~Hz}, 1 \mathrm{H}), 2.88$ (ddd, J = 16.2, $10.5,5.1 \mathrm{~Hz}$, 1H), 2.66-2.58 (m, 1H), 1.35 ppm (s, 9H); ${ }^{13} \mathrm{C}$ NMR (100 MHz, $\left.343 \mathrm{~K}, \mathrm{DMSO}-d_{6}\right): \delta=163.0(\mathrm{q}, J=3.2 \mathrm{~Hz}), 154.1$ (br), 153.6 , 148.0, 145.0, 140.0, 134.2, 129.5, 126.1, 124.8 (q, J = 269.7 Hz), 120.4, 119.0, 117.9 (q, J = 321.1 Hz), 78.2 (br), 52.3 (br), 50.7 

$(\mathrm{q}, J=24.3 \mathrm{~Hz}), 45.4,27.8,22.8 \mathrm{ppm} ;{ }^{19} \mathrm{~F}$ NMR $\left(376 \mathrm{MHz}, 343 \mathrm{~K}, \mathrm{DMSO}-d_{6}\right): \delta=-61.6(\mathrm{~s}, 3 \mathrm{~F}),-72.6 \mathrm{ppm}(\mathrm{s}, 3 \mathrm{~F})$; IR (film): $\widetilde{v}=3421$ (br), 3018, 2978, 1699, 1653, 1596, 1504, 1476, 1424, 1369, 1251, 1214, 1141, 1107, 1019, 887, 789, 753 cm-1; HRMS (ESI): $m / z$ calculated for $\mathrm{C}_{23} \mathrm{H}_{23} \mathrm{~F}_{6} \mathrm{~N}_{3} \mathrm{O}_{6} \mathrm{SNa}[\mathrm{M}+\mathrm{Na}]^{+}: 606.1104$, found: $606.1100 ;[\alpha]_{D}^{23}=-50.0\left(c=0.65, \mathrm{CHCl}_{3}, 98 \%\right.$ ee sample); HPLC analysis (CHIRALPAK IA $(\phi=0.46 \mathrm{~cm} \times 25 \mathrm{~cm}), 2$-propanol $/ n$-hexane $=1 / 9$, flow rate $=1.0 \mathrm{~mL} / \mathrm{min}$, detection at $254 \mathrm{~nm}, \mathrm{tr}_{\mathrm{R}}=11.4 \mathrm{~min}$ (major), $12.6 \mathrm{~min}$ (minor)):
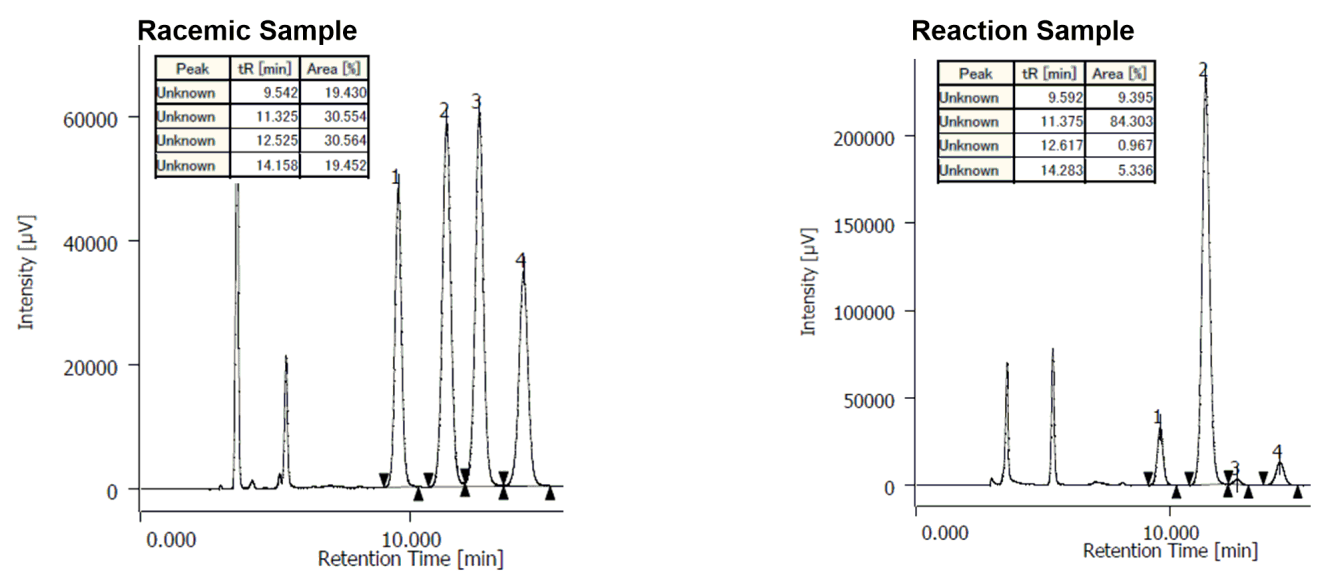

tert-Butyl ((1S,2S)-2-(2,3-dihydro-1H-pyrrolo[2,3-b]pyridine-1-carbonyl)-3,3,3-trifluoro-1-(furan-2-yl)propyl)-

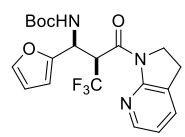
carbamate (3o).

The reaction was performed according to procedure A using N-Boc imine 20 (39.0 $\mathrm{mg}$ ) to afford $39.9 \mathrm{mg}$ (94\%) of the desired pure product 30 after purification by flash column chromatography (hexane/ethyl acetate, $2: 1)$.

White solid, m. p.: $128-130{ }^{\circ} \mathrm{C}$; ${ }^{1} \mathrm{H}$ NMR (400 MHz, $\left.343 \mathrm{~K}, \mathrm{DMSO}-\mathrm{d}_{6}\right): \delta=8.15$ (d, J=4.4 Hz, $\left.1 \mathrm{H}\right), 7.68$ (dd, J = 7.4, $1.3 \mathrm{~Hz}$, 1H), 7.50 (brs, 1H), $7.36(\mathrm{~d}, J=1.1 \mathrm{~Hz}, 1 \mathrm{H}), 7.07(\mathrm{dd}, J=7.3,5.0 \mathrm{~Hz}, 1 \mathrm{H}), 6.46-6.37(\mathrm{~m}, 1 \mathrm{H}), 6.22(\mathrm{dd}, J=3.2,1.8 \mathrm{~Hz}, 1 \mathrm{H})$, $6.10(\mathrm{~d}, J=3.4 \mathrm{~Hz}, 1 \mathrm{H}), 5.44$ (brs, 1H), $3.95(\mathrm{ddd}, J=14.8,7.7,4.4 \mathrm{~Hz}, 1 \mathrm{H}), 3.77$ (ddd, J=14.3, 8.2, 3.7 Hz, 1H), 3.06-2.89 (m, 2H), 1.38 ppm (s, 9H); ${ }^{13} \mathrm{C}$ NMR (150 MHz, $\left.343 \mathrm{~K}, \mathrm{DMSO}-d_{6}\right): \delta=162.8$ (q, J = 2.9 Hz), 154.2 (br), 154.1, $152.3,145.2$, 141.6, 134.3, 126.4, $124.4(\mathrm{q}, J=281.8 \mathrm{~Hz}), 118.9,110.0,106.2,78.1,49.2(\mathrm{q}, J=21.7 \mathrm{~Hz}), 46.4$ (br), 45.7, $27.8,23.1 \mathrm{ppm} ;{ }^{19} \mathrm{~F}$ NMR (376 MHz, 343 K, DMSO-d6): $\delta=-62.0$ ppm (s); IR (film): $\widetilde{v}=3348,2978,2928,1712,1665,1594,1501,1478,1426$, 1367, 1309, 1257, 1155, 1122, 1011, 886, 865, $787 \mathrm{~cm}^{-1}$; HRMS (ESI): $m / z$ calculated for $\mathrm{C}_{20} \mathrm{H}_{22} \mathrm{~F}_{3} \mathrm{~N}_{3} \mathrm{O}_{4} \mathrm{Na} \mathrm{MM}+\mathrm{Na}^{+}: 448.1455$, found: 448.1445; $[\alpha]_{D}^{24}=-64.6\left(c=0.45, \mathrm{CHCl}_{3}, 99 \%\right.$ ee sample); HPLC analysis (CHIRALPAK IE $(\phi=0.46 \mathrm{~cm} \times 25 \mathrm{~cm})$, 2-propanol $/ n$-hexane $=1 / 9$, flow rate $=1.0 \mathrm{~mL} / \mathrm{min}$, detection at $254 \mathrm{~nm}, \mathrm{t}_{\mathrm{R}}=46.5 \mathrm{~min}$ (major), $60.7 \mathrm{~min}(\mathrm{minor})$ ): 

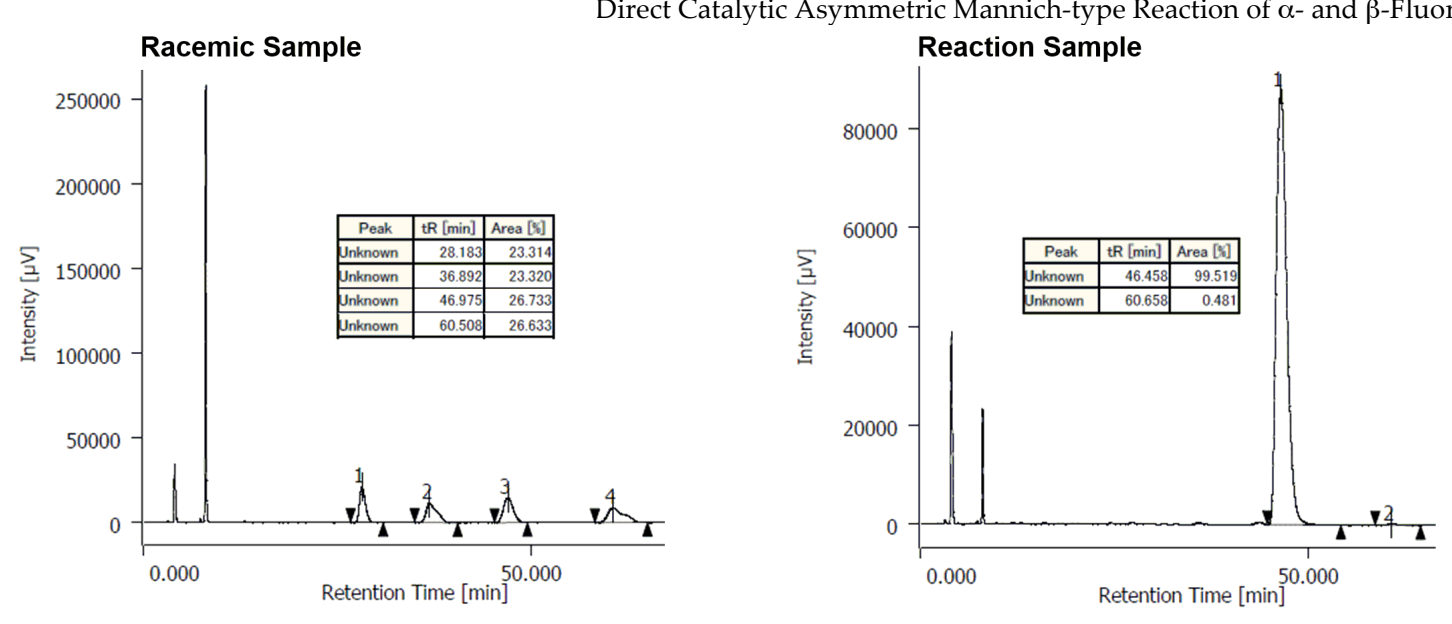

tert-Butyl ((1S,2S)-2-(2,3-dihydro-1H-pyrrolo[2,3-b]pyridine-1-carbonyl)-3,3,3-trifluoro-1-(furan-3-yl)propyl)-

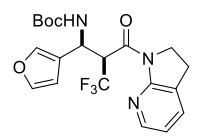
carbamate (3p).

The reaction was performed according to procedure A using $\left[\mathrm{Cu}\left(\mathrm{CH}_{3} \mathrm{CN}\right)_{4}\right] \mathrm{PF}_{6}(1.1 \mathrm{mg}, 3.0 \mu \mathrm{mol}, 0.75$ mol\%), (R)-(-)-2,2'-bis[di(3,5-diisopropyl-4-dimethylaminophenyl)phosphine]-6,6'-dimethoxy-1,1'-biphenyl (DIPAMeO-Biphep) (3.5 mg, $3.2 \mu \mathrm{mol}, 0.8 \mathrm{~mol} \%)$, anhydrous THF (0.17 mL, $2.0 \mathrm{M})$, 1-(2,3-dihydro-1H-pyrrolo[2,3-b]pyridin-1-yl)-3,3,3-trifluoropropan-1-one (1a) (92.1 mg, $0.4 \mathrm{mmol}, 1.0$ equiv.), N-Boc imine 2p (156 mg, $0.8 \mathrm{mmol}, 2.0$ equiv.), and Barton's base (0.1 M in THF, $30 \mu \mathrm{L}, 3.0 \mu \mathrm{mol}, 0.75 \mathrm{~mol} \%) .158 \mathrm{mg}$ (93\%) of the desired pure product $3 \mathrm{p}$ were obtained after purification by flash column chromatography (hexane/ethyl acetate, 2:1).

White solid, m. p.: $132-134{ }^{\circ} \mathrm{C} ;{ }^{1} \mathrm{H}$ NMR $\left(400 \mathrm{MHz}, 343 \mathrm{~K}, \mathrm{DMSO}-d_{6}\right): \delta=8.15(\mathrm{~d}, J=5.0 \mathrm{~Hz}, 1 \mathrm{H}), 7.64(\mathrm{dd}, J=7.3,1.1 \mathrm{~Hz}$, 1H), 7.50-7.41 (m, 1H), $7.40(\mathrm{~s}, 1 \mathrm{H}), 7.35$ (s, 1H), $7.05(\mathrm{dd}, J=7.3,5.0 \mathrm{~Hz}, 1 \mathrm{H}), 6.35(\mathrm{~s}, 1 \mathrm{H}), 6.34-6.26(\mathrm{~m}, 1 \mathrm{H}), 5.29(\mathrm{brt}, J=$ $9.8 \mathrm{~Hz}, 1 \mathrm{H}), 3.92(\mathrm{ddd}, J=11.8,10.8,7.1 \mathrm{~Hz}, 1 \mathrm{H}), 3.74(\mathrm{ddd}, J=11.9,10.7,5.7 \mathrm{~Hz}, 1 \mathrm{H}), 2.98(\mathrm{ddd}, J=16.5,10.6,5.9 \mathrm{~Hz}$, 1H), $2.87(\mathrm{ddd}, J=17.3,10.4,7.3 \mathrm{~Hz}, 1 \mathrm{H}), 1.38$ ppm $(\mathrm{s}, 9 \mathrm{H}) ;{ }^{13} \mathrm{C}$ NMR $\left(100 \mathrm{MHz}, 343 \mathrm{~K}, \mathrm{DMSO}-d_{6}\right): \delta=162.7(\mathrm{q}, J=2.9$ Hz), 154.2 (br), 154.1, 145.1, 142.5, 139.6, 134.2, 126.4, 124.6 (q, J = 281.8 Hz), 123.8, 118.8, 109.3, $77.9,50.8$ (q, J = 23.3 Hz), 45.6, 44.7 (br), 27.9, 23.0 ppm; ${ }^{19} \mathrm{~F}$ NMR (376 MHz, $\left.343 \mathrm{~K}, \mathrm{DMSO}-d_{6}\right): \delta=-61.7$ ppm (s); IR (film): $\widetilde{v}=3341,3062,3004$, 2977, 2932, 1707, 1661, 1594, 1504, 1477, 1445, 1426, 1394, 1367, 1324, 1254, 1155, 1122, 1102, 1023, 875 cm-1; HRMS (ESI): $m / z$ calculated for $\mathrm{C}_{20} \mathrm{H}_{22} \mathrm{~F}_{3} \mathrm{~N}_{3} \mathrm{O}_{4} \mathrm{Na}[\mathrm{M}+\mathrm{Na}]^{+}: 448.1455$, found: $448.1440 ;[\alpha]_{D}^{26}=-62.4\left(c=1.25, \mathrm{CHCl}_{3}, 93 \%\right.$ ee sample); HPLC analysis (CHIRALPAK IE $(\phi=0.46 \mathrm{~cm} \times 25 \mathrm{~cm}), 2$-propanol $/ n$-hexane $=1 / 9$, flow rate $=1.0 \mathrm{~mL} / \mathrm{min}$, detection at $254 \mathrm{~nm}, \mathrm{t}_{\mathrm{R}}=35.0 \mathrm{~min}$ (major), $50.9 \mathrm{~min}$ (minor)):
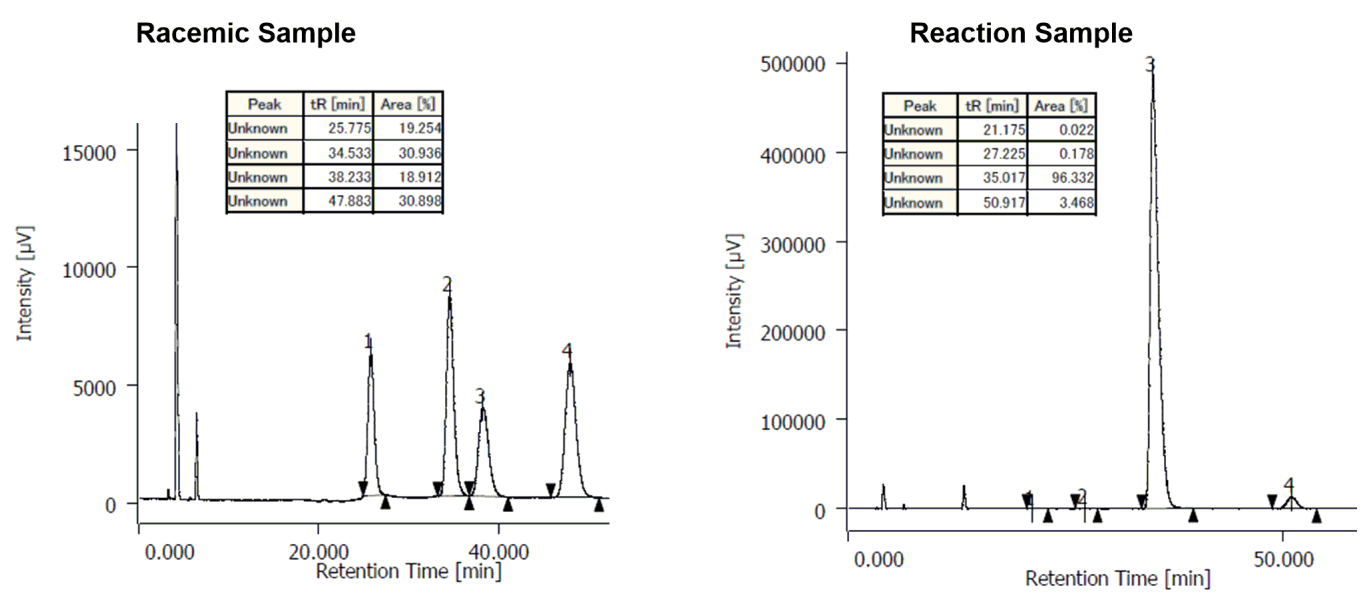

tert-Butyl ((1S,2S)-2-(2,3-dihydro-1H-pyrrolo[2,3-b]pyridine-1-carbonyl)-3,3,3-trifluoro-1-(thiophen-2-yl)propyl)-

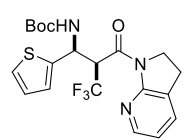
carbamate $(3 q)$.

The reaction was performed according to procedure A using $\left[\mathrm{Cu}\left(\mathrm{CH}_{3} \mathrm{CN}\right)_{4}\right] \mathrm{PF}_{6}(1.9 \mathrm{mg}, 0.005 \mathrm{mmol}$, $5 \mathrm{~mol} \%), \quad(R)-(-)-2,2$ '-bis[di(3,5-diisopropyl-4-dimethylaminophenyl)-phosphine]-6,6'-dimethoxy1,1'-biphenyl (DIPA-MeO-Biphep) (6.5 mg, $0.006 \mathrm{mmol}, 6 \mathrm{~mol} \%)$, anhydrous THF (0.15 mL, $0.5 \mathrm{M})$, N-Boc imine $2 \mathrm{q}$ (42.3 mg), and Barton's base (0.1 M in THF, $50 \mu \mathrm{L}, 0.005 \mathrm{mmol}, 5 \mathrm{~mol} \%) .40 .2 \mathrm{mg}$ (91\%) of the desired pure product $3 \mathrm{q}$ were obtained after purification by flash column chromatography (hexane/ethyl acetate, 3:1).

White solid, m. p.: $134-136{ }^{\circ} \mathrm{C}$; ${ }^{1} \mathrm{H}$ NMR $\left(400 \mathrm{MHz}, 343 \mathrm{~K}, \mathrm{DMSO}-d_{6}\right): \delta=8.16$ (s, $\left.1 \mathrm{H}\right), 7.70(\mathrm{brs}, 1 \mathrm{H}), 7.64(\mathrm{~d}, J=7.1 \mathrm{~Hz}$, 1H), $7.25(\mathrm{t}, J=3.7 \mathrm{~Hz}, 1 \mathrm{H}), 7.07-7.04(\mathrm{~m}, 1 \mathrm{H}), 6.90(\mathrm{~s}, 1 \mathrm{H}), 6.78-6.75(\mathrm{~m}, 1 \mathrm{H}), 6.51-6.42$ (m, $1 \mathrm{H}), 5.54(\mathrm{brs}, 1 \mathrm{H}), 3.94-3.87$ $(\mathrm{m}, 1 \mathrm{H}), 3.71-3.64(\mathrm{~m}, 1 \mathrm{H}), 3.00-2.92(\mathrm{~m}, 1 \mathrm{H}), 2.86-2.77(\mathrm{~m}, 1 \mathrm{H}), 1.38 \mathrm{ppm}(\mathrm{s}, 9 \mathrm{H}) ;{ }^{13} \mathrm{C} \mathrm{NMR}(150 \mathrm{MHz}, 343 \mathrm{~K}$, DMSO-d6): $\delta=163.1(q, J=2.9 \mathrm{~Hz}), 154.1(\mathrm{br}), 154.0,145.2,142.2,134.2,126.3,126.0,125.0,124.7,124.4(\mathrm{q}, J=281.8 \mathrm{~Hz})$, 118.9, 78.1, $51.0(\mathrm{q}, J=23.1 \mathrm{~Hz}), 47.9$ (br), 45.7, 27.8, 23.1 ppm; ${ }^{19} \mathrm{~F}$ NMR (376 MHz, $\left.343 \mathrm{~K}, \mathrm{DMSO}-d_{6}\right): \delta=-61.8 \mathrm{ppm}(\mathrm{s})$; IR (film): $\widetilde{v}=3341,3004,2977,2932,1708,1662,1594,1506,1476,1426,1366,1311,1255,1154,1121,1101,1043,1021,864$ $\mathrm{cm}^{-1}$; HRMS (ESI): $m / z$ calculated for $\mathrm{C}_{20} \mathrm{H}_{22} \mathrm{~F}_{3} \mathrm{~N}_{3} \mathrm{O}_{3} \mathrm{SNa}[\mathrm{M}+\mathrm{Na}]^{+}: 464.1226$, found: $464.1217 ;[\alpha]_{D}^{24}=-43.8(c=1.25$, $\mathrm{CHCl}_{3}, 94 \%$ ee sample); HPLC analysis (CHIRALPAK IC ( $\left.\phi=0.46 \mathrm{~cm} \times 25 \mathrm{~cm}\right)$, 2-propanol $/ n$-hexane $=1 / 9$, flow rate $=1.0 \mathrm{~mL} / \mathrm{min}$, detection at $254 \mathrm{~nm}, \mathrm{tr}=54.5 \mathrm{~min}$ (major), $112.8 \mathrm{~min}$ (minor)):
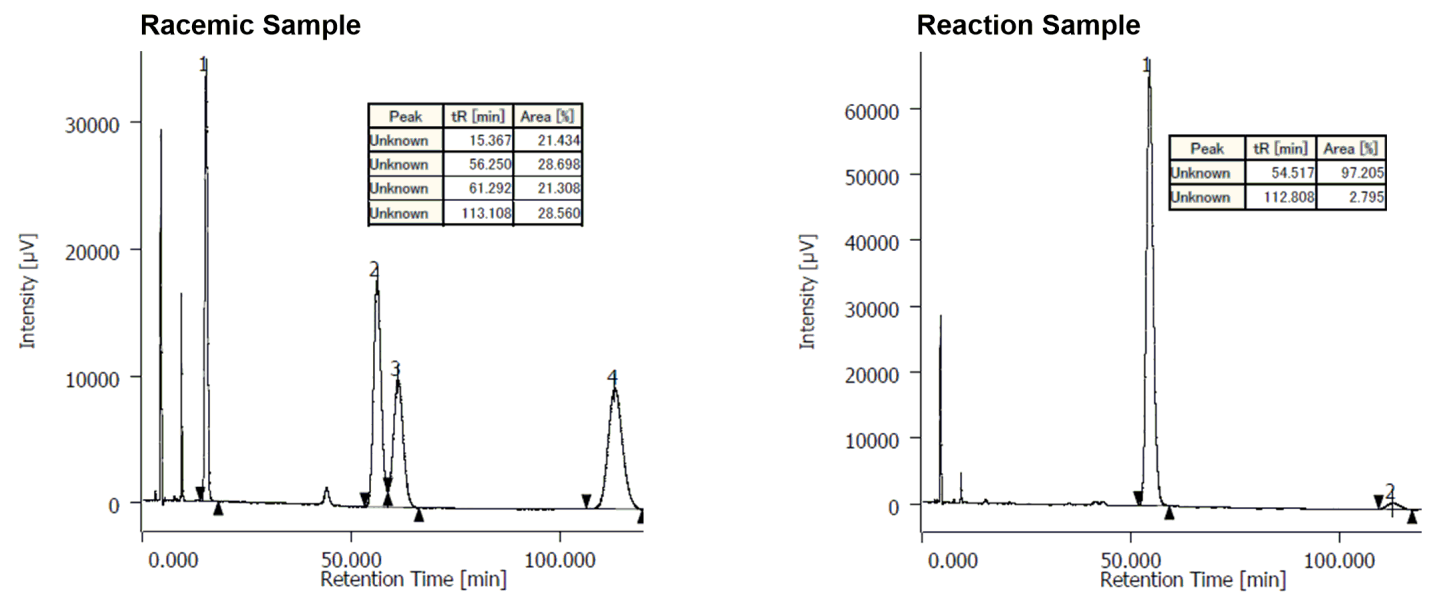

tert-Butyl ((1R,2S)-1-cyclohexyl-2-(2,3-dihydro-1H-pyrrolo[2,3-b]pyridine-1-carbonyl)-3,3,3-trifluoropropyl)-

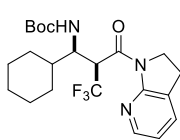
carbamate (3r).

The reaction was performed according to procedure A at a reaction temperature of $0{ }^{\circ} \mathrm{C}$ using $\mathrm{N}$-Boc imine $2 \mathrm{r}(106 \mathrm{mg}, 5.0$ equiv.) to afford $34.1 \mathrm{mg}(77 \%)$ of the desired product $3 \mathrm{r}$ as a 10:1 mixture of diastereomers after purification by flash column chromatography (hexane/ethyl acetate, 3:1). Analytical data for the major diastereomer are listed below:

White amorphous solid; ${ }^{1} \mathrm{H}$ NMR $\left(400 \mathrm{MHz}, 343 \mathrm{~K}, \mathrm{DMSO}-\mathrm{d}_{6}\right): \delta=8.11(\mathrm{~d}, J=4.1 \mathrm{~Hz}, 1 \mathrm{H}), 7.70(\mathrm{dd}, J=7.4,1.3 \mathrm{~Hz}, 1 \mathrm{H})$, $7.06(\mathrm{dd}, J=7.3,5.0 \mathrm{~Hz}, 1 \mathrm{H}), 6.63(\mathrm{brd}, J=10.3 \mathrm{~Hz}, 1 \mathrm{H}), 5.95-5.87(\mathrm{~m}, 1 \mathrm{H}), 4.22(\mathrm{td}, J=10.0,4.7 \mathrm{~Hz}, 1 \mathrm{H}), 4.08-3.96(\mathrm{~m}$, 2H), 3.12-3.05 (m, 2H), 1.67-1.40 (m, 6H), 1.38 (s, 9H), 1.16-0.90 ppm (m, 5H); ${ }^{13} \mathrm{C}$ NMR $\left(150 \mathrm{MHz}, 343 \mathrm{~K}, \mathrm{DMSO}-d_{6}\right): \delta=$ $163.9(q, J=3.4 \mathrm{~Hz}), 155.1,154.2,145.2,134.3,126.5,125.2(q, J=281.8 \mathrm{~Hz}), 118.8,77.3,52.6,48.5(q, J=23.6 \mathrm{~Hz}), 45.8,29.7$, 27.9, 27.7 (br), 26.6, 25.5, 25.4, 25.3, 23.2 ppm; ${ }^{19} \mathrm{~F}$ NMR (376 MHz, $\left.343 \mathrm{~K}, \mathrm{DMSO}-d_{6}\right): \delta=-60.9$ ppm (s); IR (film): $\widetilde{v}=3421$ 

(br), 2973, 2927, 2856, 1703, 1665, 1594, 1506, 1446, 1425, 1365, 1256, 1159, 784, 755, $689 \mathrm{~cm}^{-1}$; HRMS (ESI): m/z calculated for $\mathrm{C}_{22} \mathrm{H}_{30} \mathrm{~F}_{3} \mathrm{~N}_{3} \mathrm{O}_{3} \mathrm{Na}[\mathrm{M}+\mathrm{Na}]^{+}: 464.2131$, found: 464.2123; $[\alpha]_{D}^{24}=-32.2\left(c=0.80, \mathrm{CHCl}_{3}, 96 \%\right.$ ee sample); HPLC analysis $($ CHIRALPAK IA $(\phi=0.46 \mathrm{~cm} \times 25 \mathrm{~cm}), 2$-propanol $/ n$-hexane $=1 / 2$, flow rate $=0.5 \mathrm{~mL} / \mathrm{min}$, detection at $254 \mathrm{~nm}$, $\mathrm{t} R=$ $17.9 \min$ (major), 21.7 min (minor)):
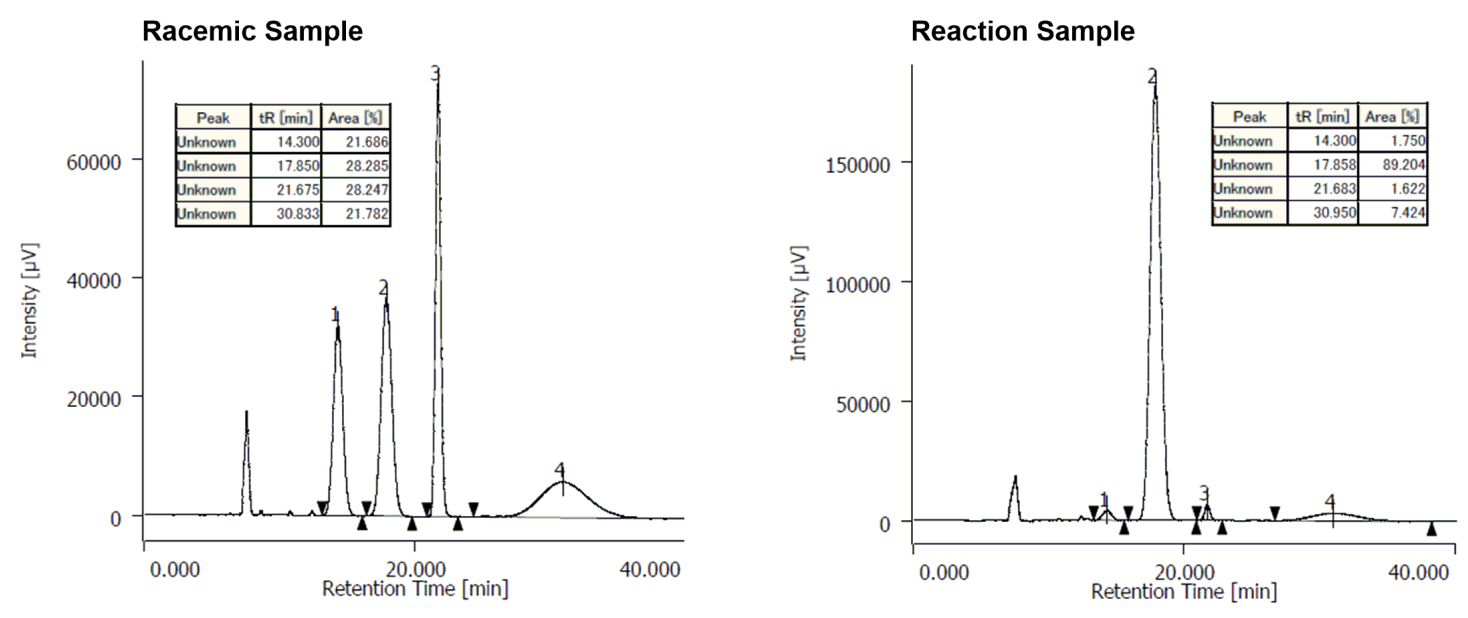

\section{tert-Butyl ((2S,3R)-2-(2,3-dihydro-1H-pyrrolo[2,3-b]pyridine-1-carbonyl)-1,1,1-trifluorooctan-3-yl)carbamate (3s).}

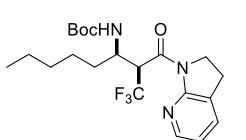

The reaction was performed according to procedure $\mathrm{A}$ at a reaction temperature of $-20{ }^{\circ} \mathrm{C}$ using $N$-Boc imine 2s (99 mg, 5.0 equiv.) to afford $34.7 \mathrm{mg}(81 \%)$ of the desired pure product 3 s after purification by flash column chromatography (hexane/ethyl acetate, 3:1).

White solid, m. p.: $118-120{ }^{\circ} \mathrm{C} ;{ }^{1} \mathrm{H}$ NMR (400 MHz, $\left.343 \mathrm{~K}, \mathrm{DMSO}-\mathrm{d}_{6}\right): \delta=8.10(\mathrm{dd}, J=4.9,1.3 \mathrm{~Hz}, 1 \mathrm{H}), 7.69(\mathrm{dq}, J=7.3$, $1.3 \mathrm{~Hz}, 1 \mathrm{H}), 7.05(\mathrm{dd}, J=7.3,5.0 \mathrm{~Hz}, 1 \mathrm{H}), 6.74(\mathrm{brd}, J=7.6 \mathrm{~Hz}, 1 \mathrm{H}), 5.95-5.86(\mathrm{~m}, 1 \mathrm{H}), 4.20-4.08(\mathrm{~m}, 1 \mathrm{H}), 4.02(\mathrm{t}, J=8.2$ $\mathrm{Hz}, 2 \mathrm{H}), 3.07(\mathrm{t}, J=8.5 \mathrm{~Hz}, 2 \mathrm{H}), 1.52-1.40(\mathrm{~m}, 1 \mathrm{H}), 1.36(\mathrm{~s}, 9 \mathrm{H}), 1.30-1.07(\mathrm{~m}, 7 \mathrm{H}), 0.80 \mathrm{ppm}(\mathrm{t}, J=6.6 \mathrm{~Hz}, 3 \mathrm{H}) ;{ }^{13} \mathrm{C} \mathrm{NMR}$ (150 MHz, $\left.343 \mathrm{~K}, \mathrm{DMSO}-d_{6}\right): \delta=163.8$ (q, $\left.J=2.9 \mathrm{~Hz}\right), 154.8,154.4,145.1,134.2,126.5,124.9$ (q, J = 281.3 Hz), $118.7,77.3$, $50.6(q, J=23.1 \mathrm{~Hz}), 48.3,45.7,31.3,30.2,27.9,24.4,23.1,21.5,13.3$ ppm; ${ }^{19} \mathrm{~F}$ NMR $\left(376 \mathrm{MHz}, 343 \mathrm{~K}, \mathrm{DMSO}-d_{6}\right): \delta=-61.4$ ppm (s); IR (film): $\widetilde{v}=3355,2960,2932,2860,1710,1665,1593,1502,1479,1424,1391,1365,1256,1160,1099,866,785$ $\mathrm{cm}^{-1}$; HRMS (ESI): $m / z$ calculated for $\mathrm{C}_{21} \mathrm{H}_{30} \mathrm{~F}_{3} \mathrm{~N}_{3} \mathrm{O}_{3} \mathrm{Na}[\mathrm{M}+\mathrm{Na}]^{+}: 452.2131$, found: $452.2119 ;[\alpha]_{D}^{24}=-50.0\left(c=0.52, \mathrm{CHCl}_{3}\right.$, 98\% ee sample); HPLC analysis (CHIRALPAK IA $(\phi=0.46 \mathrm{~cm} \mathrm{x} 25 \mathrm{~cm})$ and IA-3 $(\phi=0.46 \mathrm{~cm} \times 25 \mathrm{~cm})$, 2-propanol $/ n$-hexane $=1 / 20$, flow rate $=1.0 \mathrm{~mL} / \mathrm{min}$, detection at $254 \mathrm{~nm}, \mathrm{t}_{\mathrm{R}}=17.6 \mathrm{~min}$ (minor), $25.8 \mathrm{~min}(\mathrm{major})$ ):
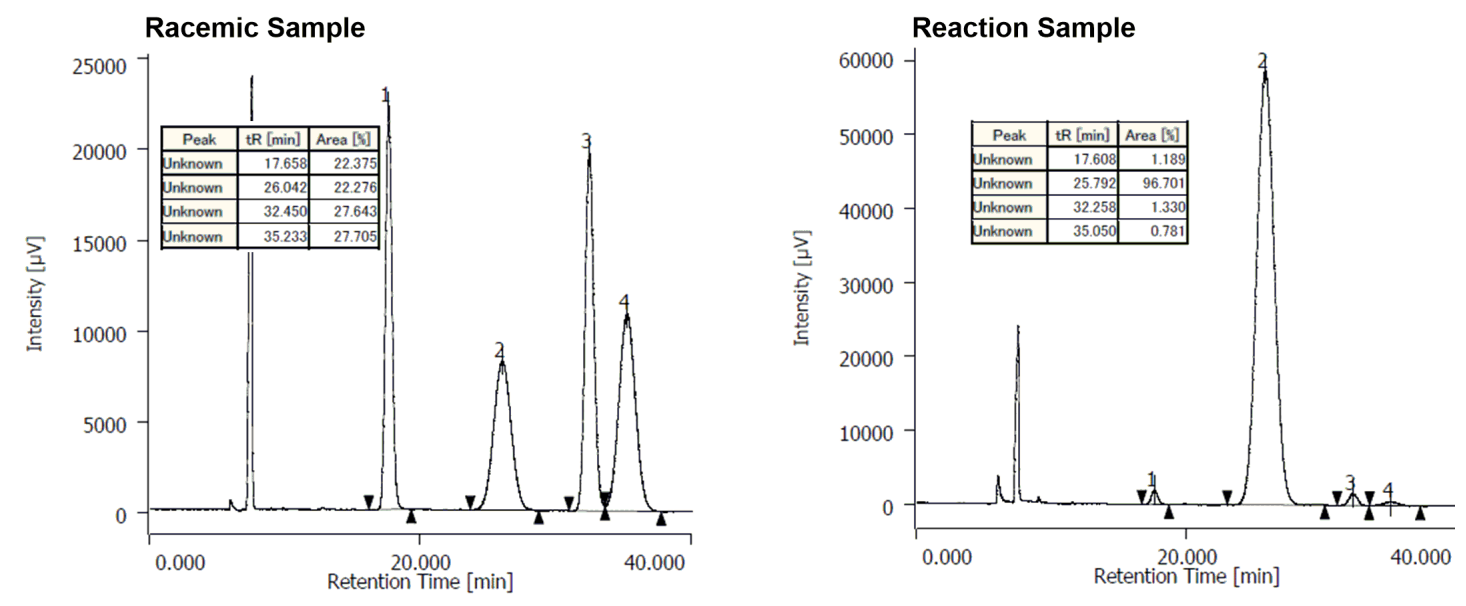
BocHN O carbamate (3t).

The reaction was performed according to procedure A at a reaction temperature of $-20{ }^{\circ} \mathrm{C}$ using $N$-Boc imine $2 \mathbf{t}$ (85 $\mathrm{mg}, 5.0$ equiv.) to afford $33.5 \mathrm{mg}(84 \%)$ of the desired pure product $3 \mathbf{t}$ after purification by flash column chromatography (hexane/ethyl acetate, 2:1).

Clear colorless oil; ${ }^{1} \mathrm{H}$ NMR (400 MHz, $\left.353 \mathrm{~K}, \mathrm{DMSO}-\mathrm{d}_{6}\right): \delta=8.11$ (d, J = 5.0 Hz, 1H), 7.69 (dd, J = 7.4, $\left.1.3 \mathrm{~Hz}, 1 \mathrm{H}\right), 7.06$ $(\mathrm{dd}, J=7.3,5.2 \mathrm{~Hz}, 1 \mathrm{H}), 6.59(\mathrm{brd}, J=8.2 \mathrm{~Hz}, 1 \mathrm{H}), 5.98-5.89(\mathrm{~m}, 1 \mathrm{H}), 4.26(\mathrm{td}, J=10.2,4.4 \mathrm{~Hz}, 1 \mathrm{H}), 4.08-3.97(\mathrm{~m}, 2 \mathrm{H})$, $3.07(\mathrm{t}, J=8.5 \mathrm{~Hz}, 2 \mathrm{H}), 1.76-1.68(\mathrm{~m}, 1 \mathrm{H}), 1.39(\mathrm{~s}, 9 \mathrm{H}), 0.84(\mathrm{t}, J=6.6 \mathrm{~Hz}, 3 \mathrm{H}), 0.71 \mathrm{ppm}(\mathrm{t}, J=6.6 \mathrm{~Hz}, 3 \mathrm{H}) ;{ }^{13} \mathrm{C} \mathrm{NMR}(150$ MHz, $\left.343 \mathrm{~K}, \mathrm{DMSO}-d_{6}\right): \delta=163.9(\mathrm{q}, J=3.4 \mathrm{~Hz}), 155.2,154.2,145.1,134.3,126.5,125.1(\mathrm{q}, J=282.2 \mathrm{~Hz}), 118.8,77.3,52.8$, $49.0(\mathrm{q}, J=23.1 \mathrm{~Hz}), 45.7,29.5,27.9,27.7$ (br), 23.1, 19.6, 16.2 ppm; ${ }^{19} \mathrm{~F}$ NMR $(376 \mathrm{MHz}, 353 \mathrm{~K}, \mathrm{DMSO}-d 6): \delta=-60.9$ ppm (s); IR (film): $\widetilde{v}=3459$ (br), 2969, 2932, 2876, 1705, 1662, 1593, 1506, 1477, 1445, 1425, 1406, 1391, 1366, 1310, 1297, 1255, 1158, 1104, 1016, 994, 865, 785, $756 \mathrm{~cm}^{-1}$; HRMS (ESI): $\mathrm{m} / z$ calculated for $\mathrm{C}_{19} \mathrm{H}_{26} \mathrm{~F}_{3} \mathrm{~N}_{3} \mathrm{O}_{3} \mathrm{Na}[\mathrm{M}+\mathrm{Na}]^{+}: 424.1818$, found: 424.1820; $[\alpha]_{D}^{23}=-43.3\left(c=1.25, \mathrm{CHCl}_{3}, 94 \%\right.$ ee sample); HPLC analysis (CHIRALPAK IA $(\phi=0.46 \mathrm{~cm} \times 25 \mathrm{~cm})$ and IA-3 $(\phi=0.46 \mathrm{~cm} \times 25 \mathrm{~cm}), 2$-propanol $/ n$-hexane $=1 / 9$, flow rate $=0.5 \mathrm{~mL} / \mathrm{min}$, detection at $254 \mathrm{~nm}$, tR $=24.5 \mathrm{~min}$ (minor), $30.3 \mathrm{~min}$ (major)):
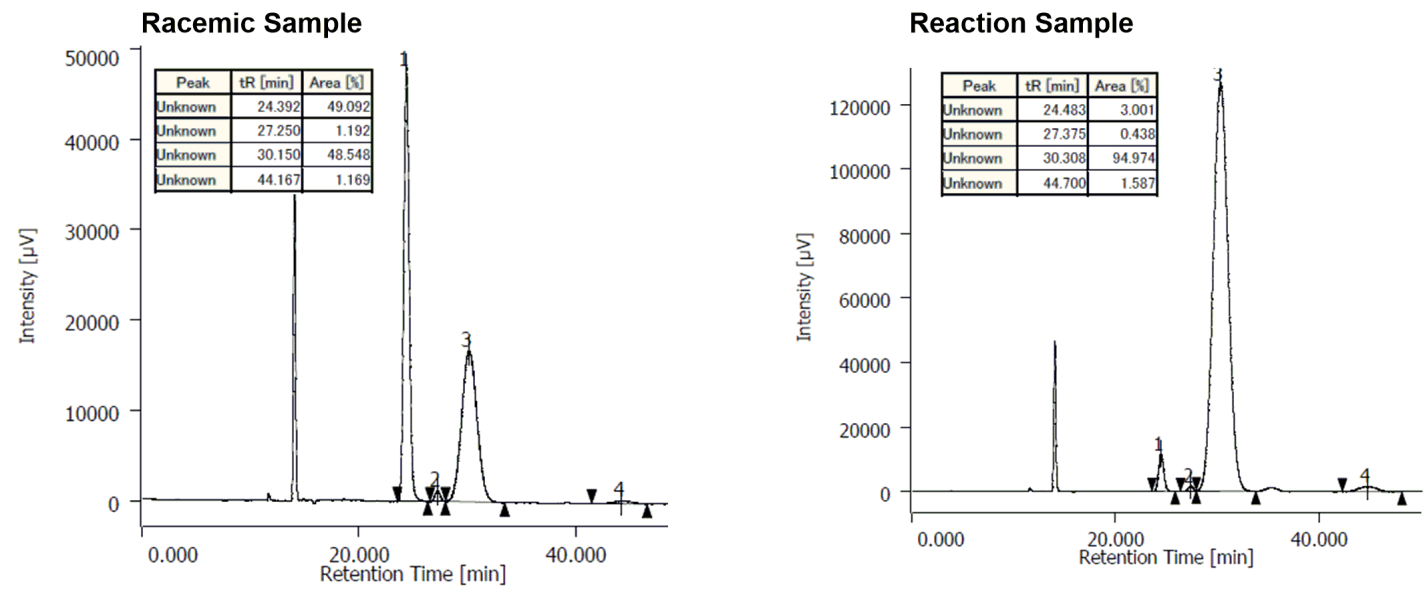

\section{4-4. Direct catalytic asymmetric Mannich-type reaction of $\alpha-\mathrm{F} \alpha-\mathrm{CF}_{3}$ amide $1 \mathrm{~b}$.}

Procedure B:

A flame-dried $20 \mathrm{~mL}$ test tube equipped with a magnetic stirring bar and a 3-way glass stopcock was charged with $\left[\mathrm{Cu}\left(\mathrm{CH}_{3} \mathrm{CN}\right)_{4}\right] \mathrm{PF}_{6}(3.7 \mathrm{mg}, 0.01 \mathrm{mmol}, 10 \mathrm{~mol} \%)$ and $(R)-(-)-2,2$ '-bis[di(3,5-diisopropyl-4-dimethylaminophenyl)phosphine]-6,6'-dimethoxy-1,1'-biphenyl (DIPA-MeO-BIPHEP) (13.1 mg, $0.012 \mathrm{mmol}, 12 \mathrm{~mol} \%)$ in a glove box under Ar atmosphere. Afterwards, the test tube was removed from the glove box and evacuated for 15 min. Then, it was backfilled with Ar and anhydrous THF $(0.10 \mathrm{~mL}, 0.5 \mathrm{M})$ was added at room temperature. The resulting clear colorless solution was stirred for $1 \mathrm{~h}$ before racemic 1-(2,3-dihydro-1H-pyrrolo[2,3-b]pyridin-1-yl)-2,3,3,3-tetrafluoropropan-1-one ( $r a c-1 b) ~(24.8 \mathrm{mg}, 0.1 \mathrm{mmol}, 1.0$ equiv.), the corresponding $\mathrm{N}$-Boc or $\mathrm{N}-\mathrm{Cbz}$ imine (2a, 

2b, 2e-2h, 2j, 2k, 2m, 2o-2q) (0.2 mmol, 2.0 equiv.) and Barton's base (a 0.1 M stock solution of Barton's base was prepared by diluting the commercial pure amine with the appropriate amount of freshly dried anhydrous THF and used for a maximum time period of 2 weeks before it was discarded; $0.1 \mathrm{M}$ in THF, $0.1 \mathrm{~mL}, 0.01 \mathrm{mmol}, 10 \mathrm{~mol} \%$ ) were subsequently added. The reaction mixture was stirred at ambient temperature until the complete consumption of the amide starting material was observed by TLC analysis $(<24 \mathrm{~h})$. The diastereomeric ratio and the enantiomeric excess of the target material were determined by HPLC analysis of an aliquot, which was taken from the reaction mixture and filtered through a short pad of silica; the diastereomeric ratio is reported if it is worse than 20:1. Then, the reaction mixture was loaded directly onto a silica gel column and eluted with a suitable solution of $n$-hexane and ethyl acetate. Evaporation of solvent afforded the pure product.

Note that the NMR spectra of the Mannich products were recorded at elevated temperature (usually $\left.70{ }^{\circ} \mathrm{C}\right)$ in DMSO-d6, as rotamers were observed in $\mathrm{CDCl}_{3}$ at room temperature. ${ }^{10}$

\section{tert-Butyl ((1R,2R)-2-(2,3-dihydro-1H-pyrrolo[2,3-b]pyridine-1-carbonyl)-2,3,3,3-tetrafluoro-1-phenylpropyl)-}

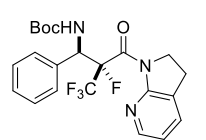
carbamate (6a).

The reaction was performed according to procedure $\mathrm{B}$ using $\left[\mathrm{Cu}\left(\mathrm{CH}_{3} \mathrm{CN}\right)_{4}\right] \mathrm{PF}_{6}(1.9 \mathrm{mg}, 0.005 \mathrm{mmol}$, $5 \mathrm{~mol} \%), \quad(R)-(-)-2,2$ '-bis[di(3,5-diisopropyl-4-dimethylaminophenyl)phosphine]-6,6'-dimethoxy1,1'-biphenyl (DIPAMeO-Biphep) (6.5 mg, $0.006 \mathrm{mmol}, 6 \mathrm{~mol} \%)$, anhydrous THF (0.15 mL, $0.5 \mathrm{M})$, N-Boc imine 2a (41.0 mg), and Barton's base $(0.1 \mathrm{M}$ in THF, $50 \mu \mathrm{L}, 0.005 \mathrm{mmol}, 5 \mathrm{~mol} \%) .42 .2 \mathrm{mg}(93 \%)$ of the desired pure product $6 \mathbf{a}$ were obtained after purification by flash column chromatography (hexane/ethyl acetate, 2:1).

White solid, m. p.: $135-137{ }^{\circ} \mathrm{C} ;{ }^{1} \mathrm{H}$ NMR (400 MHz, $\left.343 \mathrm{~K}, \mathrm{DMSO}-d_{6}\right): \delta=8.15(\mathrm{~d}, J=5.0 \mathrm{~Hz}, 1 \mathrm{H}), 7.88(\mathrm{brs}, 1 \mathrm{H})$, 7.60-7.57 (m, 1H), 7.51-7.45 (m, 2H), 7.32-7.27 (m, 3H), $7.04(\mathrm{dd}, J=7.3,5.0 \mathrm{~Hz}, 1 \mathrm{H}), 6.00(\mathrm{brdd}, J=26.8,9.9 \mathrm{~Hz}, 1 \mathrm{H})$, 3.83-3.75 (m, 1H), 3.50-3.41 (m, 1H), $2.82(\mathrm{ddd}, J=16.0,9.9,6.0 \mathrm{~Hz}, 1 \mathrm{H}), 2.69-2.60(\mathrm{~m}, 1 \mathrm{H}), 1.37 \mathrm{ppm}(\mathrm{s}, 9 \mathrm{H}) ;{ }^{13} \mathrm{C} \mathrm{NMR}$ (125 MHz, 343 K, DMSO-d6): $\delta=161.1$ (d, J = 21.4 Hz), 155.2, 154.3 (br), 145.7, 135.1, 133.2, 128.3, 128.0, 127.9, 125.6, 121.6 $(\mathrm{qd}, J=288.0,30.5 \mathrm{~Hz}), 119.6,96.7(\mathrm{dq}, J=216.3,26.9 \mathrm{~Hz}), 78.6,55.1(\mathrm{brm}), 47.2(\mathrm{~d}, J=12.1 \mathrm{~Hz}), 27.8,25.2$ ppm; ${ }^{19} \mathrm{~F}$ NMR (376 MHz, $\left.343 \mathrm{~K}, \mathrm{DMSO}-d_{6}\right): \delta=-66.0$ (s, 3F), -173.1 ppm (brs, 1F); IR (film): $\widetilde{v}=3337$ (br), 3062, 2983, 2928, 1709, 1682, $1596,1497,1420,1392,1368,1345,1308,1257,1207,1166,1002,755 \mathrm{~cm}^{-1}$; HRMS (ESI): $m / z$ calculated for $\mathrm{C}_{22} \mathrm{H}_{24} \mathrm{~F}_{4} \mathrm{~N}_{3} \mathrm{O}_{3}$ $[\mathrm{M}+\mathrm{H}]^{+}: 454.1748$, found: $454.1743 ;[\alpha]_{D}^{26}=+66.4\left(c=1.25, \mathrm{CHCl}_{3}, 95 \%\right.$ ee sample); HPLC analysis (CHIRALPAK ID $(\phi=0.46 \mathrm{~cm} \times 25 \mathrm{~cm}), 2$-propanol $/ n$-hexane $=1 / 2$, flow rate $=1.0 \mathrm{~mL} / \mathrm{min}$, detection at $254 \mathrm{~nm}, \mathrm{t} \mathrm{R}=16.2 \mathrm{~min}(\mathrm{major}), 25.2$ $\min (\operatorname{minor}))$ : 

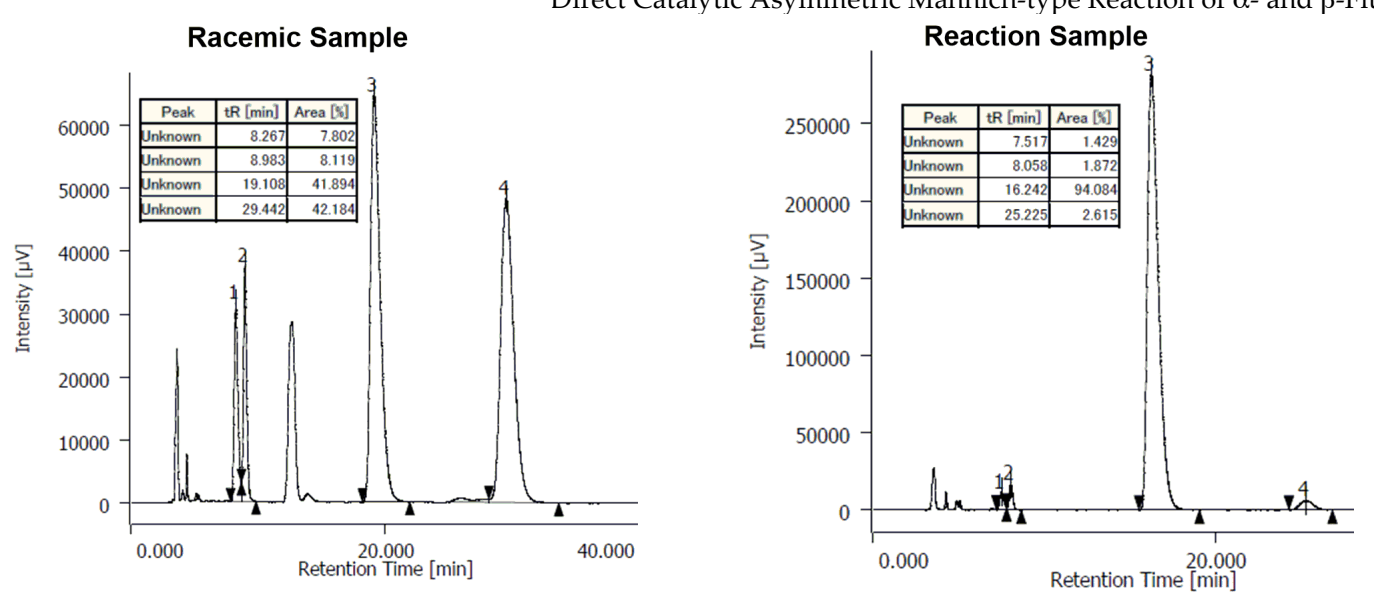

Benzyl ((1R,2R)-2-(2,3-dihydro-1H-pyrrolo[2,3-b]pyridine-1-carbonyl)-2,3,3,3-tetrafluoro-1-phenylpropyl)carbamate

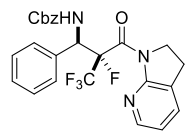
(6b).

The reaction was performed according to procedure B using $\mathrm{N}-\mathrm{Cbz}$ imine $\mathbf{2 b}(47.8 \mathrm{mg})$ to afford $41.3 \mathrm{mg}$ $(88 \%)$ of the desired pure product $6 \mathbf{b}$ after purification by flash column chromatography (hexane/ethyl acetate, $2: 1)$. White solid, m. p.: $139-140{ }^{\circ} \mathrm{C} ;{ }^{1} \mathrm{H} \mathrm{NMR}\left(400 \mathrm{MHz}, 343 \mathrm{~K}, \mathrm{DMSO}-d_{6}\right): \delta=8.43$ (brs, $\left.1 \mathrm{H}\right), 8.15(\mathrm{~d}, J=4.5 \mathrm{~Hz}, 1 \mathrm{H})$, 7.60-7.57 (m, 1H), 7.51-7.46 (m, 2H), 7.36-7.27 (m, 8H), $7.03(\mathrm{dd}, J=7.4,5.0 \mathrm{~Hz}, 1 \mathrm{H}), 6.07$ (brdd, $J=26.1,7.5 \mathrm{~Hz}, 1 \mathrm{H})$, $5.11(\mathrm{~d}, J=12.7 \mathrm{~Hz}, 1 \mathrm{H}), 5.04(\mathrm{~d}, J=12.6 \mathrm{~Hz}, 1 \mathrm{H}), 3.83-3.75(\mathrm{~m}, 1 \mathrm{H}), 3.49-3.41(\mathrm{~m}, 1 \mathrm{H}), 2.81(\mathrm{ddd}, J=16.2,9.9,6.1 \mathrm{~Hz}$, 1H), 2.64 ppm (ddd, $J=16.5,9.1,6.9 \mathrm{~Hz}, 1 \mathrm{H}) ;{ }^{13} \mathrm{C}$ NMR (125 MHz, $\left.343 \mathrm{~K}, \mathrm{DMSO}-d_{6}\right): \delta=161.0(\mathrm{brd}, J=21.7 \mathrm{~Hz}), 155.2$ (br), 145.7, 136.4, 134.9, 133.2, 128.3 (2C), 128.2, 128.0 (5C), 127.5, 127.2 (2C), 125.6, 121.6 (qd, J = 287.8, 30.4 Hz), 119.7, $96.7(\mathrm{dq}, J=218.7,27.4 \mathrm{~Hz}), 65.7,55.7(\mathrm{~d}, J=18.7 \mathrm{~Hz}), 47.3(\mathrm{~d}, J=12.1 \mathrm{~Hz}), 25.2 \mathrm{ppm} ;{ }^{19} \mathrm{~F} \mathrm{NMR}(376 \mathrm{MHz}, 343 \mathrm{~K}$, DMSO-d6): $\delta=-70.6$ (s, 3F), -178.2 ppm (brs, 1F); IR (film): $\widetilde{v}=3324$ (br), 3066, 3035, 2983, 2922, 1710, 1679, 1596, 1537, 1420, 1395, 1345, 1306, 1258, 1210, 1182, 1010, 753, $701 \mathrm{~cm}^{-1}$; HRMS (ESI): m/z calculated for $\mathrm{C}_{25} \mathrm{H}_{22} \mathrm{~F}_{4} \mathrm{~N}_{3} \mathrm{O}_{3}[\mathrm{M}+\mathrm{H}]^{+}$: 488.1592, found: 488.1587; $[\alpha]_{D}^{25}=+44.3\left(c=1.20, \mathrm{CHCl}_{3}, 90 \%\right.$ ee sample); HPLC analysis (CHIRALPAK ID $(\phi=0.46 \mathrm{~cm}$ x $25 \mathrm{~cm}$ ), 2-propanol $/ n$-hexane $=1 / 2$, flow rate $=1.0 \mathrm{~mL} / \mathrm{min}$, detection at $254 \mathrm{~nm}, \mathrm{t}_{\mathrm{R}}=54.5 \mathrm{~min}(\mathrm{major}), 75.4 \mathrm{~min}$ (minor)):
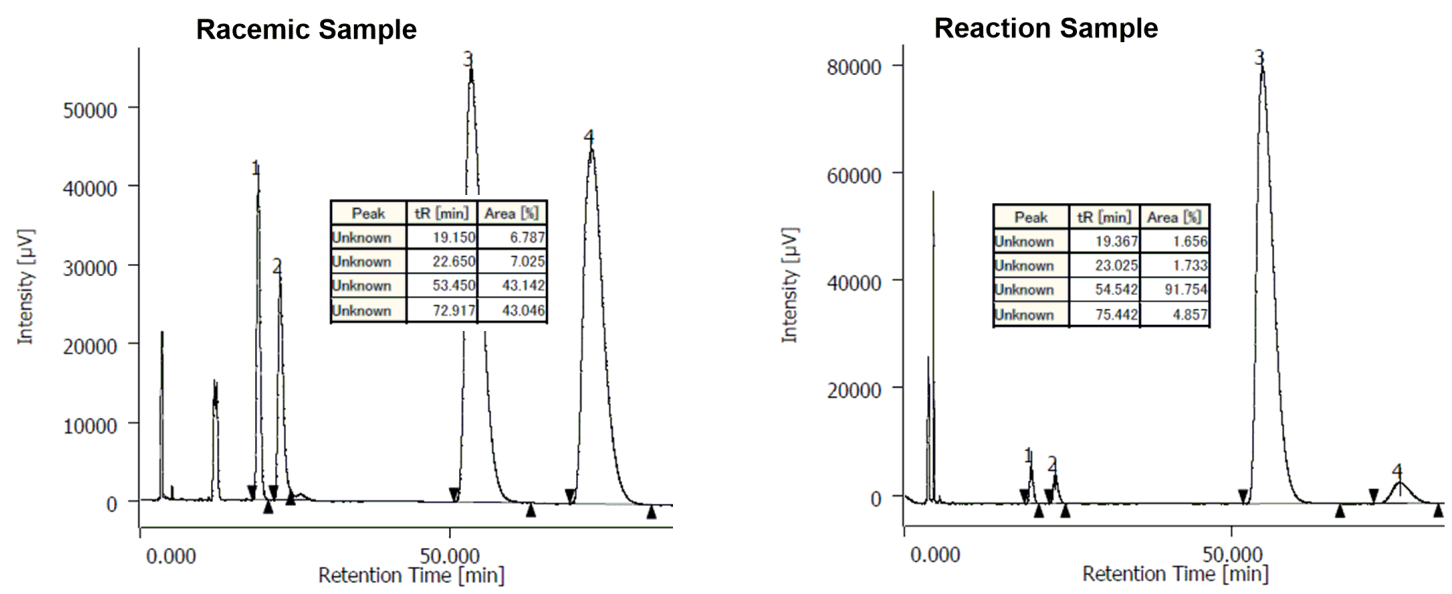

tert-Butyl ((1R,2R)-2-(2,3-dihydro-1H-pyrrolo[2,3-b]pyridine-1-carbonyl)-2,3,3,3-tetrafluoro-1-(para-tolyl)propyl)-

BocHN Oll carbamate (6e).

The reaction was performed according to procedure B using $N$-Boc imine $2 \mathbf{e}(43.9 \mathrm{mg})$ to afford $42.0 \mathrm{mg}$ (90\%) of the desired pure product 6 e after purification by flash column chromatography (hexane/ethyl acetate, 3:2).

White solid, m. p.: $162-164{ }^{\circ} \mathrm{C}$; ${ }^{1} \mathrm{H}$ NMR (400 MHz, $343 \mathrm{~K}$, DMSO-d6): $\delta=8.16-8.14(\mathrm{~m}, 1 \mathrm{H}), 7.81$ (brs, $\left.1 \mathrm{H}\right), 7.61-7.58$ (m, $1 \mathrm{H}), 7.35(\mathrm{~d}, J=7.8 \mathrm{~Hz}, 2 \mathrm{H}), 7.10(\mathrm{~d}, J=7.8 \mathrm{~Hz}, 2 \mathrm{H}), 7.04(\mathrm{dd}, J=7.4,5.0 \mathrm{~Hz}, 1 \mathrm{H}), 5.95(\mathrm{brdd}, J=26.7,9.7 \mathrm{~Hz}, 1 \mathrm{H})$, 3.83-3.76 (m, 1H), 3.51-3.43 (m, 1H), $2.83(\mathrm{ddd}, J=16.2,9.7,5.7 \mathrm{~Hz}, 1 \mathrm{H}), 2.64(\mathrm{ddd}, J=16.8,8.7,7.6 \mathrm{~Hz}, 1 \mathrm{H}), 2.25(\mathrm{~s}, 3 \mathrm{H})$, 1.37 ppm (s, 9H); ${ }^{13} \mathrm{C}$ NMR (125 MHz, $\left.343 \mathrm{~K}, \mathrm{DMSO}-d_{6}\right): \delta=161.2$ (d, J = 21.9 Hz), 155.3, 154.3 (br), $145.7,137.4,133.2$, 132.2, 128.4, 128.2, 125.6, $121.6(\mathrm{qd}, J=287.8,30.5 \mathrm{~Hz}), 119.6,96.7(\mathrm{dq}, J=218.6,27.2 \mathrm{~Hz}), 78.6,54.8(\mathrm{brd}, J=13.0 \mathrm{~Hz}), 47.2$ $(\mathrm{d}, J=12.1 \mathrm{~Hz}), 27.8,25.2,20.2 \mathrm{ppm} ;{ }^{19} \mathrm{~F}$ NMR (376 MHz, $\left.343 \mathrm{~K}, \mathrm{DMSO}-d_{6}\right): \delta=-70.7$ (s, 3F), -178.0 ppm (brs, $\left.1 \mathrm{~F}\right) ; \mathrm{IR}$ (film): $\widetilde{v}=3341$ (br), 3059, 3004, 2979, 2925, 1709, 1683, 1596, 1507, 1421, 1392, 1368, 1343, 1307, 1254, 1207, 1167, 1124, 1007, $758 \mathrm{~cm}^{-1}$; HRMS (ESI): $m / z$ calculated for $\mathrm{C}_{23} \mathrm{H}_{26} \mathrm{~F}_{4} \mathrm{~N}_{3} \mathrm{O}_{3}[\mathrm{M}+\mathrm{H}]^{+}: 468.1905$, found: $468.1897 ;[\alpha]_{D}^{26}=+58.4(c=1.15$, $\mathrm{CHCl}_{3}, 97 \%$ ee sample); HPLC analysis (CHIRALPAK ID $(\phi=0.46 \mathrm{~cm} \times 25 \mathrm{~cm})$, 2-propanol $/ n$-hexane $=1 / 2$, flow rate $=1.0 \mathrm{~mL} / \mathrm{min}$, detection at $254 \mathrm{~nm}, \mathrm{t}_{\mathrm{R}}=23.9 \mathrm{~min}$ (major), $42.8 \mathrm{~min}$ (minor)):
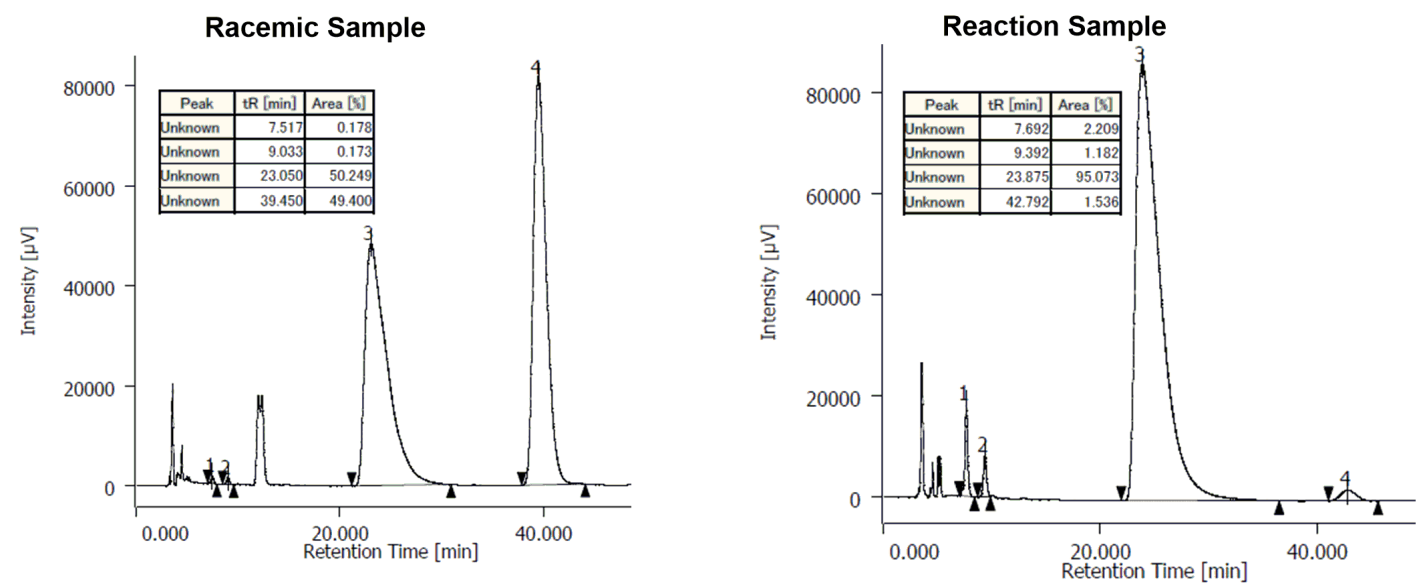

\section{tert-Butyl ((1R,2R)-2-(2,3-dihydro-1H-pyrrolo[2,3-b]pyridine-1-carbonyl)-2,3,3,3-tetrafluoro-1-(3-vinylphenyl)-}

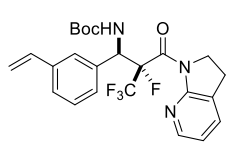

\section{propyl)carbamate (6f).}

The reaction was performed according to procedure B using N-Boc imine $2 \mathrm{f}$ (46.3 $\mathrm{mg}$ ) to afford 46.2 mg (96\%) of the desired product $6 f$ as a 20:1 mixture of diastereomers after purification by flash column chromatography (hexane/ethyl acetate, 2:1). Analytical data for the major diastereomer are listed below:

White amorphous solid; ${ }^{1} \mathrm{H}$ NMR $\left(400 \mathrm{MHz}, 343 \mathrm{~K}, \mathrm{DMSO}-d_{6}\right): \delta=8.16-8.14(\mathrm{~m}, 1 \mathrm{H}), 7.90(\mathrm{brs}, 1 \mathrm{H}), 7.59-7.57(\mathrm{~m}, 2 \mathrm{H})$, 7.38-7.35 (m, 2H), $7.27(\mathrm{t}, J=7.6 \mathrm{~Hz}, 1 \mathrm{H}), 7.04(\mathrm{dd}, J=7.4,5.0 \mathrm{~Hz}, 1 \mathrm{H}), 6.65(\mathrm{dd}, J=17.6,10.9 \mathrm{~Hz}, 1 \mathrm{H}), 6.02(\mathrm{brdd}, J=26.1$, 9.6 Hz, 1H), $5.73(\mathrm{dd}, J=17.6,0.9 \mathrm{~Hz}, 1 \mathrm{H}), 5.22(\mathrm{dd}, J=10.9,0.9 \mathrm{~Hz}, 1 \mathrm{H}), 3.84-3.76(\mathrm{~m}, 1 \mathrm{H}), 3.52-3.44(\mathrm{~m}, 1 \mathrm{H}), 2.82(\mathrm{ddd}$, $J=16.1,9.7,6.1 \mathrm{~Hz}, 1 \mathrm{H}), 2.66(\mathrm{ddd}, J=16.6,9.1,6.8 \mathrm{~Hz}, 1 \mathrm{H}), 1.38$ ppm $(\mathrm{s}, 9 \mathrm{H}) ;{ }^{13} \mathrm{C}$ NMR $\left(125 \mathrm{MHz}, 343 \mathrm{~K}, \mathrm{DMSO}-d_{6}\right): \delta=$ $161.1(\mathrm{~d}, J=21.7 \mathrm{~Hz}), 155.2,154.4(\mathrm{br}), 145.7,137.0,135.9,135.4,133.2,128.1,127.8,125.9(2), 125.9,125.6,121.6(\mathrm{qd}, J=$ 287.8, 30.5 Hz), 119.6, 114.4, $96.6(\mathrm{dq}, J=219.4,26.8 \mathrm{~Hz}), 78.7,55.0(\mathrm{brd}, J=14.4 \mathrm{~Hz}), 47.3$ (d, J = 11.8 Hz), $27.8,25.1$ ppm; ${ }^{19} \mathrm{~F}$ NMR (376 MHz, $\left.343 \mathrm{~K}, \mathrm{DMSO}-d_{6}\right): \delta=-70.7$ (s, 3F), -177.8 ppm (brs, 1F); IR (film): $\widetilde{v}=3355$ (br), $3059,3007,2980$, 1707, 1682, 1596, 1507, 1420, 1392, 1368, 1342, 1307, 1254, 1206, 1166, 1009, 912, 872, 762 cm ${ }^{-1}$; HRMS (ESI): m/z 

calculated for $\mathrm{C}_{24} \mathrm{H}_{26} \mathrm{~F}_{4} \mathrm{~N}_{3} \mathrm{O}_{3}[\mathrm{M}+\mathrm{H}]^{+}: 480.1905$, found: $480.1898 ;[\alpha]_{D}^{26}=+66.0$ (c=0.90, $\mathrm{CHCl}_{3}, 92 \%$ ee sample); HPLC analysis (CHIRALPAK ID $(\phi=0.46 \mathrm{~cm} \times 25 \mathrm{~cm})$, 2-propanol $/ n$-hexane $=1 / 2$, flow rate $=1.0 \mathrm{~mL} / \mathrm{min}$, detection at 254 $\mathrm{nm}, \mathrm{t}_{\mathrm{R}}=13.0 \mathrm{~min}$ (major), $64.1 \mathrm{~min}($ minor)):
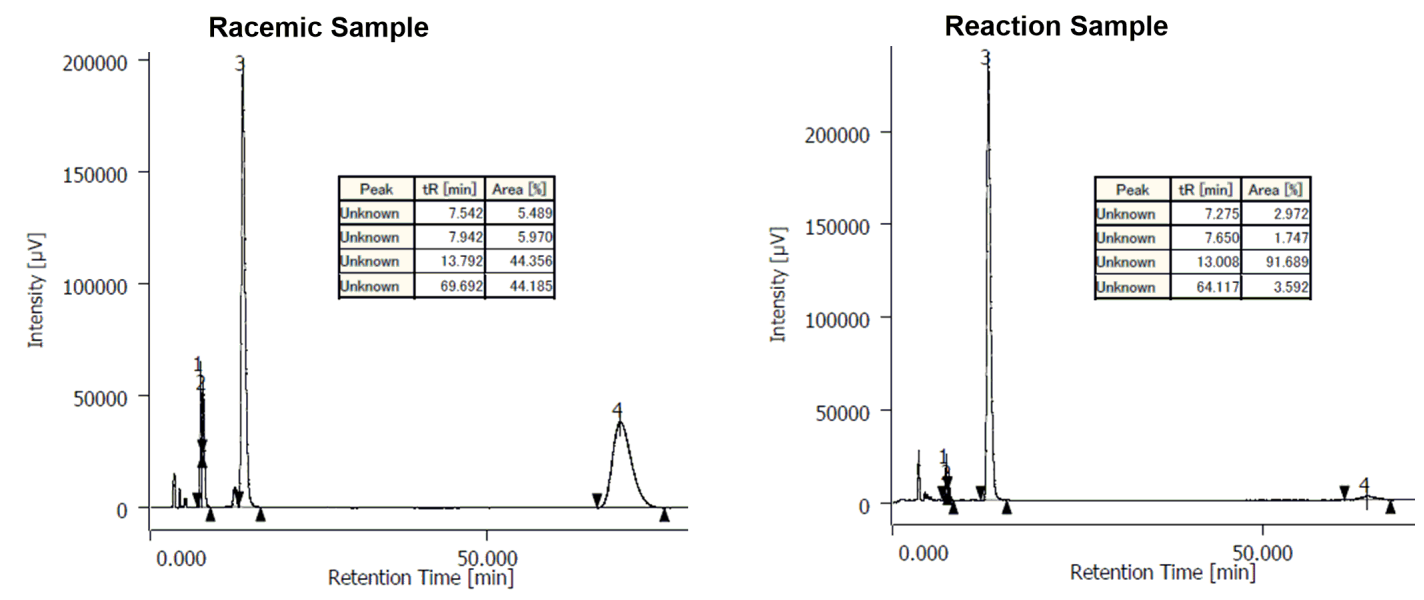

tert-Butyl ((1R,2R)-2-(2,3-dihydro-1H-pyrrolo[2,3-b]pyridine-1-carbonyl)-2,3,3,3-tetrafluoro-1-(3-methoxyphenyl)-

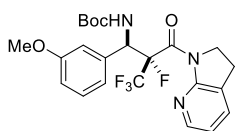
propyl)carbamate $(6 \mathrm{~g})$.

The reaction was performed according to procedure B using $N$-Boc imine $2 \mathrm{~g}$ (47.1 $\mathrm{mg}$ ) to afford 46.9 $\mathrm{mg}(97 \%)$ of the desired product $6 \mathrm{~g}$ as a $17: 1$ mixture of diastereomers after purification by flash column chromatography (hexane/ethyl acetate, 2:1). Analytical data for the major diastereomer are listed below: White amorphous solid; ${ }^{1} \mathrm{H}$ NMR (400 MHz, $343 \mathrm{~K}$, DMSO-d6): $\delta=8.16-8.15$ (m, 1H), 7.89 (brs, $\left.1 \mathrm{H}\right), 7.60(\mathrm{dd}, J=7.4,1.2$ $\mathrm{Hz}, 1 \mathrm{H}), 7.20(\mathrm{t}, J=7.9 \mathrm{~Hz}, 1 \mathrm{H}), 7.09(\mathrm{~s}, 1 \mathrm{H}), 7.06-7.01(\mathrm{~m}, 2 \mathrm{H}), 6.85(\mathrm{ddd}, J=8.2,2.5,0.8 \mathrm{~Hz}, 1 \mathrm{H}), 5.97(\mathrm{brdd}, J=26.9,9.3$ $\mathrm{Hz}, 1 \mathrm{H}), 3.86-3.78(\mathrm{~m}, 1 \mathrm{H}), 3.68(\mathrm{~s}, 3 \mathrm{H}), 3.53-3.44(\mathrm{~m}, 1 \mathrm{H}), 2.85(\mathrm{ddd}, J=16.2,9.6,5.8 \mathrm{~Hz}, 1 \mathrm{H}), 2.67(\mathrm{ddd}, J=16.6,9.0,7.3$ Hz, 1H), 1.38 ppm (s, 9H); ${ }^{13} \mathrm{C}$ NMR (125 MHz, $\left.343 \mathrm{~K}, \mathrm{DMSO}-\mathrm{d}_{6}\right): \delta=161.1$ (d, J = 21.5 Hz), 158.9, 155.3, 154.3 (br), 145.7 , 136.5, 133.2, 128.9, 125.6, 121.6 (qd, $J=287.9,30.4$ Hz), 120.6, 119.6, 114.0, 113.9, 96.7 (dq, J = 220.5, 26.8 Hz), 78.6, 55.1 (brd, $J=17.2 \mathrm{~Hz}), 54.9,47.2(\mathrm{~d}, J=12.4 \mathrm{~Hz}), 27.8,25.2 \mathrm{ppm} ;{ }^{19} \mathrm{~F}$ NMR (376 MHz, $\left.343 \mathrm{~K}, \mathrm{DMSO}-d_{6}\right): \delta=-70.8(\mathrm{~s}, 3 \mathrm{~F})$, -177.7 ppm (brs, 1F); IR (film): $\widetilde{v}=3337$ (br), 3059, 2979, 2932, 1708, 1682, 1599, 1493, 1420, 1392, 1368, 1343, 1291, 1253, 1205, 1166, 1044, 1009, 868, $768 \mathrm{~cm}^{-1}$; HRMS (ESI): $m / z$ calculated for $\mathrm{C}_{23} \mathrm{H}_{26} \mathrm{~F}_{4} \mathrm{~N}_{3} \mathrm{O}_{4}[\mathrm{M}+\mathrm{H}]^{+}:$484.1854, found: 484.1844; $[\alpha]_{D}^{26}=+66.6\left(c=0.85, \mathrm{CHCl}_{3}, 92 \%\right.$ ee sample); HPLC analysis (CHIRALPAK IC $(\phi=0.46 \mathrm{~cm} \times 25 \mathrm{~cm})$, 2-propanol $/ n$-hexane $=1 / 4$, flow rate $=1.0 \mathrm{~mL} / \mathrm{min}$, detection at $254 \mathrm{~nm}, \mathrm{t}_{\mathrm{R}}=71.6 \mathrm{~min}$ (major), $84.6 \mathrm{~min}(\mathrm{minor})$ ):
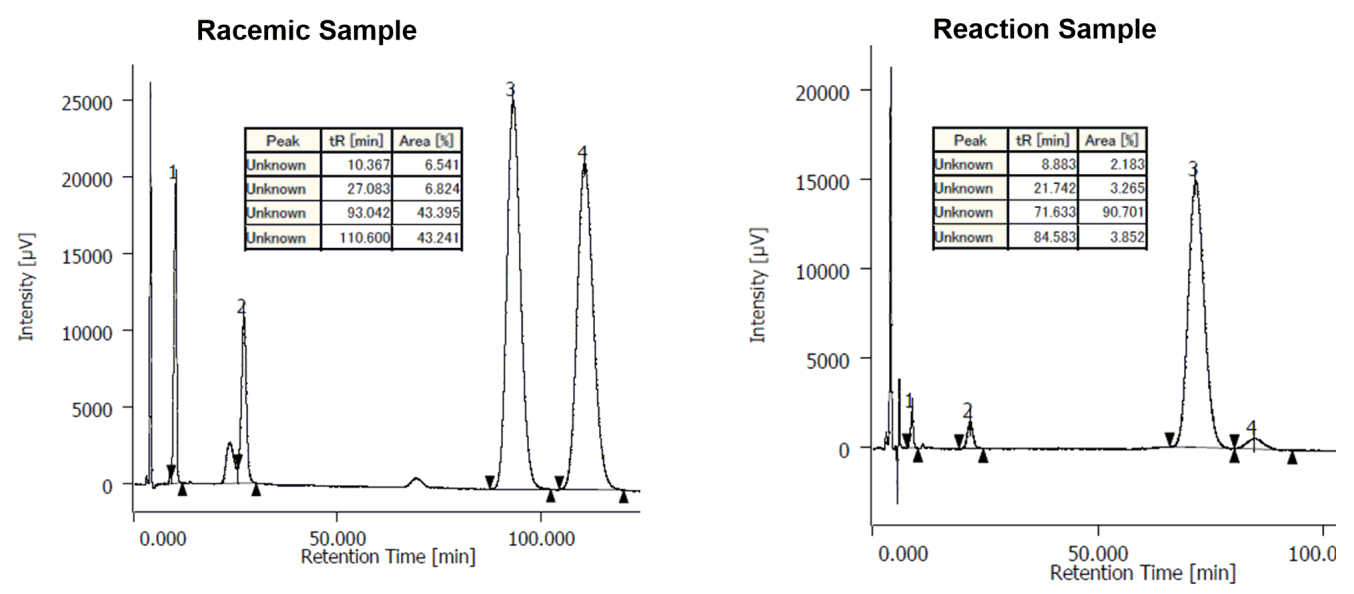

tert-Butyl ((1R,2R)-2-(2,3-dihydro-1H-pyrrolo[2,3-b]pyridine-1-carbonyl)-2,3,3,3-tetrafluoro-1-(4-methoxyphenyl)-

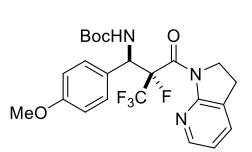

\section{propyl)carbamate (6h).}

The reaction was performed according to procedure B using $\left[\mathrm{Cu}\left(\mathrm{CH}_{3} \mathrm{CN}\right)_{4}\right] \mathrm{PF}_{6}(1.9 \mathrm{mg}, 0.005 \mathrm{mmol}$,

5 mol\%), (R)-(-)-2,2'-bis[di(3,5-diisopropyl-4-dimethylaminophenyl)phosphine]-6,6'-dimethoxy1,1'-biphenyl (DIPA-MeO-Biphep) $(6.5 \mathrm{mg}, 0.006 \mathrm{mmol}, 6 \mathrm{~mol} \%)$, anhydrous THF (0.20 mL, $0.4 \mathrm{M})$, N-Boc imine $2 \mathrm{~h}$ (47.1 mg), and Barton's base (0.1 M in THF, $50 \mu \mathrm{L}, 0.005 \mathrm{mmol}, 5 \mathrm{~mol} \%) .34 .2 \mathrm{mg}(73 \%)$ of the desired pure product $6 \mathbf{h}$ were obtained along with unreacted starting material after purification by flash column chromatography (hexane/ethyl acetate, 3:2).

White amorphous solid; ${ }^{1} \mathrm{H}$ NMR (400 MHz, $\left.343 \mathrm{~K}, \mathrm{DMSO}-d_{6}\right): \delta=8.16-8.14(\mathrm{~m}, 1 \mathrm{H}), 7.80$ (brs, $\left.1 \mathrm{H}\right), 7.61-7.58(\mathrm{~m}, 1 \mathrm{H})$, $7.39(\mathrm{~d}, J=8.3 \mathrm{~Hz}, 2 \mathrm{H}), 7.04(\mathrm{dd}, J=7.3,4.9 \mathrm{~Hz}, 1 \mathrm{H}), 6.87-6.83(\mathrm{~m}, 2 \mathrm{H}), 5.93(\mathrm{brdd}, J=27.0,10.2 \mathrm{~Hz}, 1 \mathrm{H}), 3.85-3.77(\mathrm{~m}$, 1H), $3.71(\mathrm{~s}, 3 \mathrm{H}), 3.54-3.46(\mathrm{~m}, 1 \mathrm{H}), 2.84(\mathrm{ddd}, J=16.3,9.8,5.9 \mathrm{~Hz}, 1 \mathrm{H}), 2.68(\mathrm{ddd}, J=16.5,8.9,7.7 \mathrm{~Hz}, 1 \mathrm{H}), 1.37 \mathrm{ppm}(\mathrm{s}$, 9H); ${ }^{13} \mathrm{C}$ NMR (125 MHz, $\left.343 \mathrm{~K}, \mathrm{DMSO}-d_{6}\right): \delta=161.2(\mathrm{~d}, J=21.6 \mathrm{~Hz}), 159.0,155.3,154.3$ (br), 145.7, 133.2, 129.6, 127.1, 125.6, $121.6(\mathrm{qd}, J=288.0,30.6 \mathrm{~Hz}), 119.6,113.4,96.8(\mathrm{dq}, J=217.9,27.1 \mathrm{~Hz}), 78.5,54.9,54.6(\mathrm{brd}, J=17.2 \mathrm{~Hz}), 47.2(\mathrm{~d}, J=$ $12.3 \mathrm{~Hz}), 27.8,25.3 \mathrm{ppm} ;{ }^{19} \mathrm{~F}$ NMR (376 MHz, $343 \mathrm{~K}$, DMSO-d6): $\delta=-70.7$ (s, 3F), -178.2 ppm (brs, 1F); IR (film): $\widetilde{v}=3348$ (br), 2979, 2932, 2840, 1713, 1682, 1613, 1596, 1514, 1420, 1392, 1368, 1344, 1308, 1252, 1207, 1165, 1032, 1006, $755 \mathrm{~cm}^{-1}$; HRMS (ESI): $m / z$ calculated for $\mathrm{C}_{23} \mathrm{H}_{26} \mathrm{~F}_{4} \mathrm{~N}_{3} \mathrm{O}_{4}[\mathrm{M}+\mathrm{H}]^{+}: 484.1854$, found: $484.1848 ;[\alpha]_{D}^{25}=+50.2\left(c=0.75, \mathrm{CHCl}_{3}, 99 \% e e\right.$ sample); HPLC analysis (CHIRALPAK ID $(\phi=0.46 \mathrm{~cm} \times 25 \mathrm{~cm}$ ), 2-propanol $/ n$-hexane $=1 / 2$, flow rate $=1.0 \mathrm{~mL} / \mathrm{min}$, detection at $254 \mathrm{~nm}, \mathrm{t}_{\mathrm{R}}=24.2 \mathrm{~min}$ (major), $38.8 \mathrm{~min}$ (minor)):
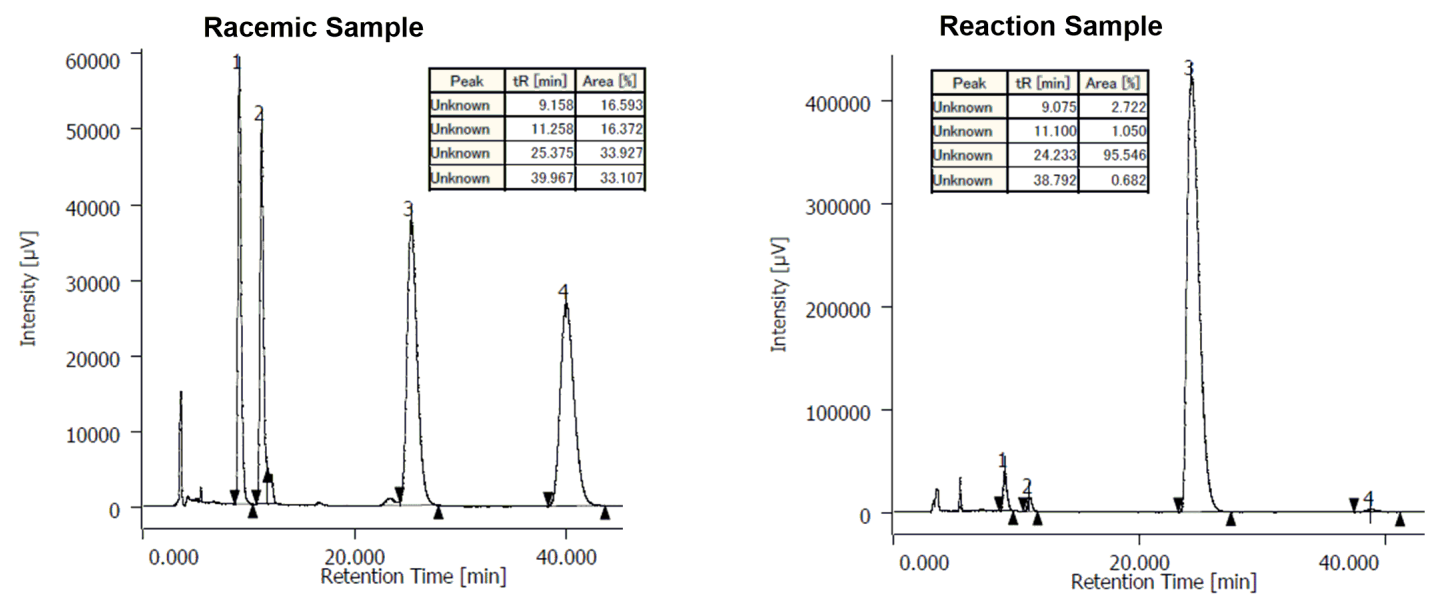

tert-Butyl ((1R,2R)-2-(2,3-dihydro-1H-pyrrolo[2,3-b]pyridine-1-carbonyl)-2,3,3,3-tetrafluoro-1-(3-fluorophenyl)-

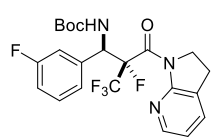
propyl)carbamate (6j).

The reaction was performed according to procedure B using N-Boc imine $2 \mathbf{j}(44.7 \mathrm{mg})$ to afford $45.0 \mathrm{mg}$ $(95 \%)$ of the desired product $\mathbf{6} \mathbf{j}$ as a 17:1 mixture of diastereomers after purification by flash column chromatography (hexane/ethyl acetate, 2:1). Analytical data for the major diastereomer are listed below:

White solid, m. p.: $158-159^{\circ} \mathrm{C}$; ${ }^{1} \mathrm{H}$ NMR (400 MHz, $\left.343 \mathrm{~K}, \mathrm{DMSO}-d_{6}\right): \delta=8.17-8.16(\mathrm{~m}, 1 \mathrm{H}), 7.91$ (brs, $\left.1 \mathrm{H}\right), 7.63-7.60$ (m, 1H), 7.39-7.28 (m, 3H), 7.13-7.08 (m, 1H), $7.06(\mathrm{dd}, J=7.4,5.0 \mathrm{~Hz}, 1 \mathrm{H}), 6.13$ (brdd, J = 24.3, $8.6 \mathrm{~Hz}, 1 \mathrm{H}), 3.90-3.82(\mathrm{~m}$, 1H), 3.64-3.57 (m, 1H), $2.90(\mathrm{ddd}, J=16.3,9.6,6.1 \mathrm{~Hz}, 1 \mathrm{H}), 2.78(\mathrm{ddd}, J=16.4,9.2,6.9 \mathrm{~Hz}, 1 \mathrm{H}), 1.37 \mathrm{ppm}(\mathrm{s}, 9 \mathrm{H}) ;{ }^{13} \mathrm{C}$ 

NMR (125 MHz, 343 K, DMSO-d6): $\delta=161.6(\mathrm{~d}, J=244.1 \mathrm{~Hz}), 160.9(\mathrm{~d}, J=22.2 \mathrm{~Hz}), 155.0,154.3(\mathrm{br}), 145.6,137.8(\mathrm{~d}, J=$ $6.4 \mathrm{~Hz}), 133.4,129.8(\mathrm{~d}, J=8.2 \mathrm{~Hz}), 125.7,124.5,121.5(\mathrm{qd}, J=288.0,30.3 \mathrm{~Hz}), 119.7,115.1(\mathrm{~d}, J=22.7 \mathrm{~Hz}), 114.9(\mathrm{~d}, J=$ $20.9 \mathrm{~Hz}), 96.3(\mathrm{dq}, J=217.3,28.0 \mathrm{~Hz}), 78.8,54.6(\mathrm{brd}, J=14.8 \mathrm{~Hz}), 47.4(\mathrm{~d}, J=10.7 \mathrm{~Hz}), 27.7,25.1 \mathrm{ppm} ;{ }^{19} \mathrm{~F} \mathrm{NMR}(376$ MHz, $\left.343 \mathrm{~K}, \mathrm{DMSO}-d_{6}\right): \delta=-70.6$ (s, 3F), -112.8 (s, 1F), -177.0 ppm (brs, 1F); IR (film): $\widetilde{v}=3344$ (br), $3069,2980,2932$, $1709,1682,1594,1490,1446,1420,1392,1368,1344,1308,1250,1204,1165,869,760 \mathrm{~cm}^{-1}$; HRMS (ESI): m/z calculated for $\mathrm{C}_{22} \mathrm{H}_{23} \mathrm{~F}_{5} \mathrm{~N}_{3} \mathrm{O}_{3}[\mathrm{M}+\mathrm{H}]^{+}:$472.1654, found: 472.1646; $[\alpha]_{D}^{25}=+50.9$ (c $=1.10, \mathrm{CHCl}_{3}, 90 \%$ ee sample); HPLC analysis $($ CHIRALPAK ID $(\phi=0.46 \mathrm{~cm} \times 25 \mathrm{~cm}), 2$-propanol $/ n$-hexane $=1 / 2$, flow rate $=1.0 \mathrm{~mL} / \mathrm{min}$, detection at $254 \mathrm{~nm}$, tR $=$ $13.8 \mathrm{~min}$ (major), $26.0 \mathrm{~min}$ (minor)):
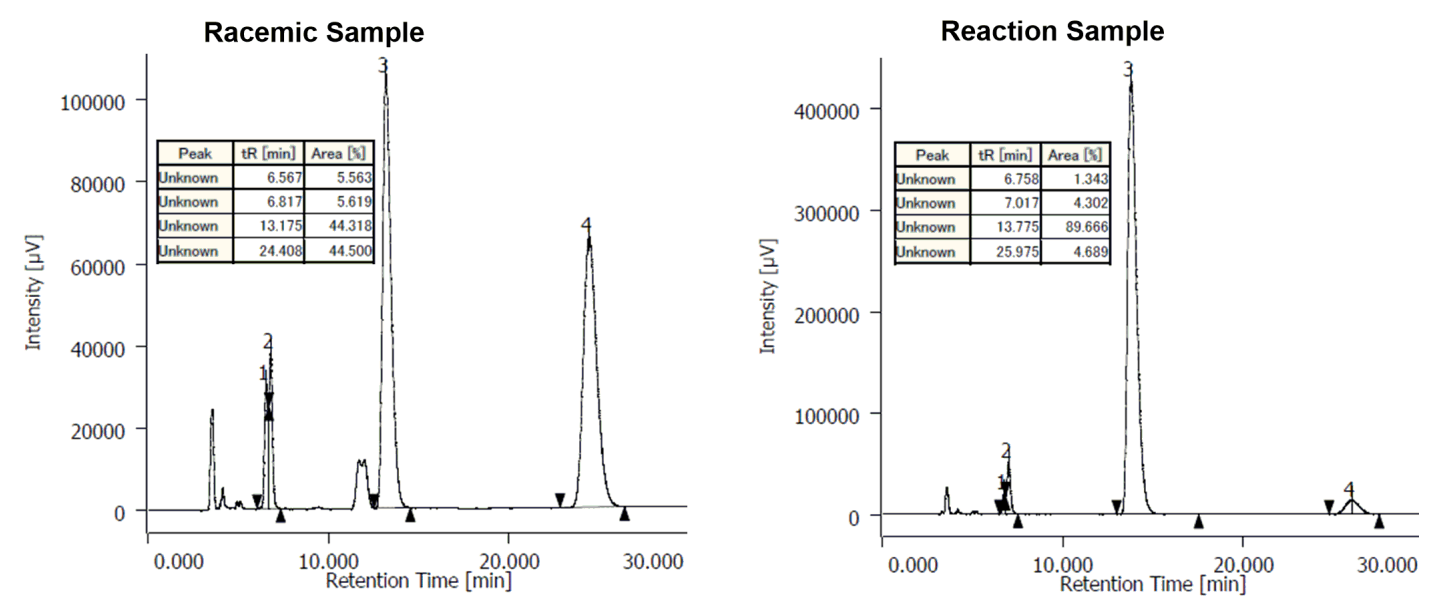

tert-Butyl ((1R,2R)-2-(2,3-dihydro-1H-pyrrolo[2,3-b]pyridine-1-carbonyl)-2,3,3,3-tetrafluoro-1-(4-fluorophenyl)-

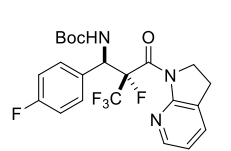
propyl)carbamate (6k).

The reaction was performed according to procedure B using N-Boc imine $2 \mathbf{k}(44.7 \mathrm{mg})$ to afford 43.0 mg (91\%) of the desired pure product $6 \mathbf{k}$ after purification by flash column chromatography (hexane/ethyl acetate, 2:1).

White amorphous solid; ${ }^{1} \mathrm{H}$ NMR (400 MHz, $343 \mathrm{~K}$, DMSO-d6): $\delta=8.17-8.15(\mathrm{~m}, 1 \mathrm{H}), 7.86(\mathrm{brs}, 1 \mathrm{H}), 7.63-7.60(\mathrm{~m}, 1 \mathrm{H})$, $7.52(\mathrm{dd}, J=8.0,5.6 \mathrm{~Hz}, 2 \mathrm{H}), 7.14-7.08(\mathrm{~m}, 2 \mathrm{H}), 7.05(\mathrm{dd}, J=7.4,5.0 \mathrm{~Hz}, 1 \mathrm{H}), 6.06(\mathrm{brdd}, J=25.6,10.0 \mathrm{~Hz}, 1 \mathrm{H}), 3.89-3.81$ (m, 1H), 3.63-3.55 (m, 1H), $2.89(\mathrm{ddd}, J=16.3,9.6,6.3 \mathrm{~Hz}, 1 \mathrm{H}), 2.77(\mathrm{ddd}, J=16.5,9.1,6.9 \mathrm{~Hz}, 1 \mathrm{H}), 1.37 \mathrm{ppm}(\mathrm{s}, 9 \mathrm{H}) ;{ }^{13} \mathrm{C}$ NMR (125 MHz, 343 K, DMSO-d6): $\delta=161.7(\mathrm{~d}, J=245.2 \mathrm{~Hz}), 161.1$ (d, $J=22.0 \mathrm{~Hz}), 155.1,154.3$ (br), $145.6,133.3,131.4$, $130.5(\mathrm{~d}, J=8.0 \mathrm{~Hz}), 125.7,121.5(\mathrm{qd}, J=287.7,30.0 \mathrm{~Hz}), 119.7,114.7(\mathrm{~d}, J=21.5 \mathrm{~Hz}), 96.5(\mathrm{dq}, J=217.7,26.3 \mathrm{~Hz}), 78.7$, $54.4(\mathrm{brd}, J=13.3 \mathrm{~Hz}), 47.4(\mathrm{~d}, J=11.2 \mathrm{~Hz}), 27.8,25.1$ ppm; ${ }^{19} \mathrm{~F}$ NMR $\left(376 \mathrm{MHz}, 343 \mathrm{~K}, \mathrm{DMSO}-d_{6}\right): \delta=-70.6(\mathrm{~s}, 3 \mathrm{~F}),-113.7$ (s, 1F), -177.5 ppm (brs, 1F); IR (film): $\widetilde{v}=3346$ (br), 2978, 2922, 1710, 1681, 1597, 1510, 1420, 1392, 1368, 1344, 1308, 1209, 1164, 1123, 1007, $771 \mathrm{~cm}^{-1}$; HRMS (ESI): $m / z$ calculated for $\mathrm{C}_{22} \mathrm{H}_{23} \mathrm{~F}_{5} \mathrm{~N}_{3} \mathrm{O}_{3}[\mathrm{M}+\mathrm{H}]^{+}: 472.1654$, found: 472.1647; $[\alpha]_{D}^{25}=$ $+61.3\left(c=0.10, \mathrm{CHCl}_{3}, 94 \%\right.$ ee sample); HPLC analysis (CHIRALPAK IE $(\phi=0.46 \mathrm{~cm} \times 25 \mathrm{~cm}), 2$-propanol $/ n$-hexane $=$ $1 / 6$, flow rate $=1.0 \mathrm{~mL} / \mathrm{min}$, detection at $254 \mathrm{~nm}, \mathrm{t}=39.6 \mathrm{~min}$ (major), $77.4 \mathrm{~min}$ (minor)): 
Racemic Sample

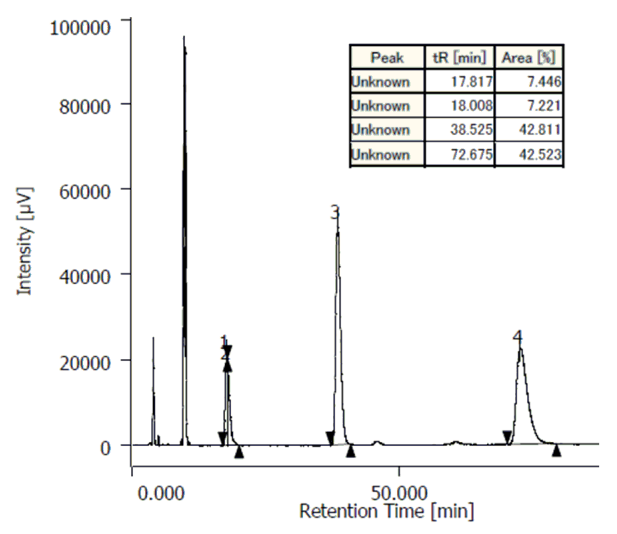

Reaction Sample

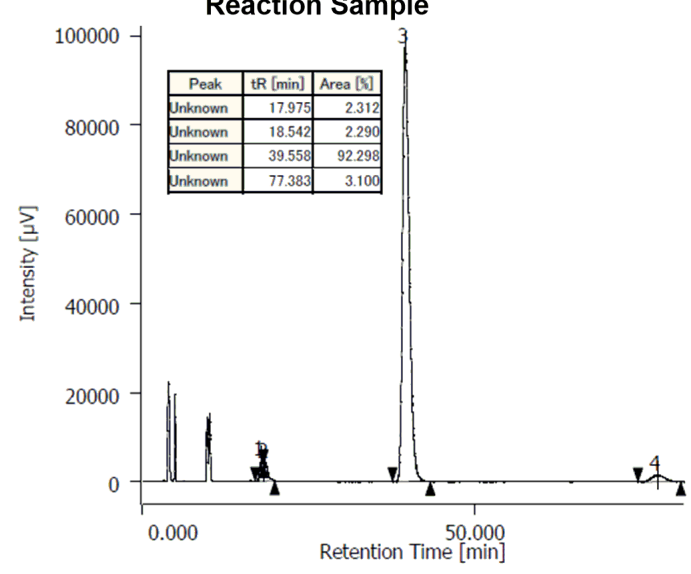

tert-Butyl ((1R,2R)-2-(2,3-dihydro-1H-pyrrolo[2,3-b]pyridine-1-carbonyl)-2,3,3,3-tetrafluoro-1-(3-nitrophenyl)-

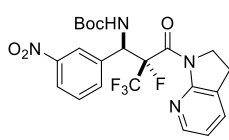
propyl)carbamate $(6 \mathrm{~m})$.

The reaction was performed according to procedure B using $N$-Boc imine $2 \mathrm{~m}(50.1 \mathrm{mg})$ at a reaction temperature of $-40{ }^{\circ} \mathrm{C} .46 .0 \mathrm{mg}(92 \%)$ of the desired pure product $6 \mathrm{~m}$ were obtained after purification by flash column chromatography (hexane/ethyl acetate, 3:2).

White amorphous solid; ${ }^{1} \mathrm{H}$ NMR (400 MHz, $343 \mathrm{~K}$, DMSO- $\left.\mathrm{d}_{6}\right): \delta=8.45(\mathrm{~s}, 1 \mathrm{H}), 8.24-8.09(\mathrm{~m}, 3 \mathrm{H}), 7.93(\mathrm{~d}, J=7.6 \mathrm{~Hz}, 1 \mathrm{H})$, 7.64-7.60 (m, 2H), $7.06(\mathrm{dd}, J=7.4,5.0 \mathrm{~Hz}, 1 \mathrm{H}), 6.35(\mathrm{brdd}, J=22.3,9.7 \mathrm{~Hz}, 1 \mathrm{H}), 3.92-3.85(\mathrm{~m}, 1 \mathrm{H}), 3.76-3.68(\mathrm{~m}, 1 \mathrm{H})$, 2.96-2.81 (m, 2H), 1.37 ppm (s, 9H); ${ }^{13} \mathrm{C}$ NMR (125 MHz, $\left.343 \mathrm{~K}, \mathrm{DMSO}-\mathrm{d}_{6}\right): \delta=160.8$ (d, $\left.J=22.1 \mathrm{~Hz}\right), 154.7,154.4$ (br), 147.6, 145.5, 137.2, 134.8, 133.5, 129.5, 125.9, 123.1, 122.9, 121.4 (qd, J = 287.9, 30.0 Hz), 119.7, 95.9 (dq, $J=217.5,27.6 \mathrm{~Hz})$, 79.0, $54.3(\mathrm{brd}, J=17.8 \mathrm{~Hz}), 47.7$ (d, $J=9.3 \mathrm{~Hz}), 27.7,24.9$ ppm; ${ }^{19} \mathrm{~F}$ NMR (376 MHz, $343 \mathrm{~K}$, DMSO-d6): $\delta=-70.4(\mathrm{~s}, 3 \mathrm{~F})$, -176.5 ppm (brs, 1F); IR (film): $\widetilde{v}=3344$ (br), 3073, 2980, 2932, 1711, 1673, 1595, 1532, 1444, 1421, 1392, 1351, 1308, 1254, 1207, 1165, 1050, 1012, 872, $757 \mathrm{~cm}^{-1}$; HRMS (ESI): $\mathrm{m} / z$ calculated for $\mathrm{C}_{22} \mathrm{H}_{23} \mathrm{~F}_{4} \mathrm{~N}_{4} \mathrm{O}_{5}[\mathrm{M}+\mathrm{H}]^{+}:$499.1599, found: 499.1596; $[\alpha]_{D}^{25}=+51.2\left(c=1.10, \mathrm{CHCl}_{3}, 93 \%\right.$ ee sample); HPLC analysis (CHIRALPAK ID $(\phi=0.46 \mathrm{~cm} \times 25 \mathrm{~cm})$, 2-propanol $/ \mathrm{n}$-hexane $=1 / 2$, flow rate $=1.0 \mathrm{~mL} / \mathrm{min}$, detection at $254 \mathrm{~nm}, \mathrm{t}=37.5 \mathrm{~min}$ (major), $77.1 \mathrm{~min}(\operatorname{minor})$ ):
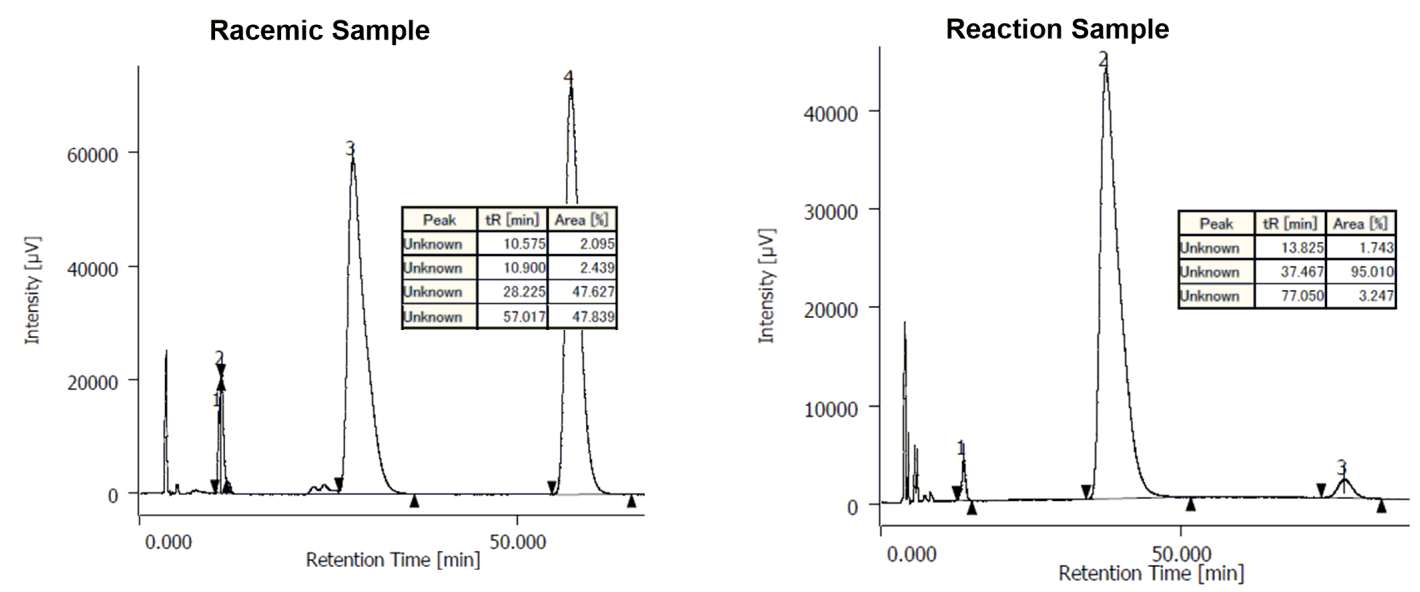

tert-Butyl ((1R,2R)-2-(2,3-dihydro-1H-pyrrolo[2,3-b]pyridine-1-carbonyl)-2,3,3,3-tetrafluoro-1-(furan-2-yl)propyl)-

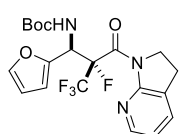
carbamate (6o).

The reaction was performed according to procedure B using $\left[\mathrm{Cu}\left(\mathrm{CH}_{3} \mathrm{CN}\right)_{4}\right] \mathrm{PF}_{6}(1.9 \mathrm{mg}, 0.005 \mathrm{mmol}$, 
(DIPA-MeO-Biphep) (6.5 mg, $0.006 \mathrm{mmol}, 6 \mathrm{~mol} \%)$, anhydrous THF (0.15 mL, $0.5 \mathrm{M})$, N-Boc imine $2 \mathrm{o}$ (39.4 mg), and Barton's base (0.1 M in THF, $50 \mu \mathrm{L}, 0.005 \mathrm{mmol}, 5 \mathrm{~mol} \%) .42 .1 \mathrm{mg}$ (95\%) of the desired pure product $60 \mathrm{were}$ obtained after purification by flash column chromatography (hexane/ethyl acetate, 3:2).

White amorphous solid; ${ }^{1} \mathrm{H}$ NMR (400 MHz, $343 \mathrm{~K}$, DMSO-d6): $\delta=8.16-8.14(\mathrm{~m}, 1 \mathrm{H}), 7.67$ (brs, $\left.1 \mathrm{H}\right), 7.66$ (dq, $J=7.4,1.3$ $\mathrm{Hz}, 1 \mathrm{H}), 7.56(\mathrm{dd}, J=1.8,0.8 \mathrm{~Hz}, 1 \mathrm{H}), 7.07(\mathrm{dd}, J=7.4,5.0 \mathrm{~Hz}, 1 \mathrm{H}), 6.47(\mathrm{~d}, J=3.3 \mathrm{~Hz}, 1 \mathrm{H}), 6.40(\mathrm{dd}, J=3.3,1.8 \mathrm{~Hz}, 1 \mathrm{H})$, 6.22 (brdd, $J=24.3,9.5 \mathrm{~Hz}, 1 \mathrm{H}), 4.02-3.94(\mathrm{~m}, 1 \mathrm{H}), 3.88-3.80(\mathrm{~m}, 1 \mathrm{H}), 3.07-2.92(\mathrm{~m}, 2 \mathrm{H}), 1.39 \mathrm{ppm}(\mathrm{s}, 9 \mathrm{H}) ;{ }^{13} \mathrm{C} \mathrm{NMR}(125$ MHz, $\left.343 \mathrm{~K}, \mathrm{DMSO}-\mathrm{d}_{6}\right): \delta=160.7$ (d, $J=22.0 \mathrm{~Hz}$ ), 155.2, 154.3 (br), 148.3, 145.6, 142.7, 133.4, 125.8, 121.4 (qd, J=287.9, $30.0 \mathrm{~Hz}), 119.6,110.4,108.9,95.5(\mathrm{dq}, J=218.1,27.8 \mathrm{~Hz}), 78.9,50.1$ (brd, $J=16.1 \mathrm{~Hz}), 47.6$ (d, $J=11.0 \mathrm{~Hz}), 27.7,25.2$ ppm; ${ }^{19} \mathrm{~F}$ NMR (376 MHz, $\left.343 \mathrm{~K}, \mathrm{DMSO}-d_{6}\right): \delta=-71.3$ (s, 3F), -176.1 ppm (brs, 1F); IR (film): $\widetilde{v}=3351$ (br), $2980,2928,1716$, 1682, 1596, 1497, 1444, 1421, 1393, 1369, 1345, 1306, 1273, 1242, 1208, 1166, 1009, 763 cm-1; HRMS (ESI): m/z calculated for $\mathrm{C}_{20} \mathrm{H}_{22} \mathrm{~F}_{4} \mathrm{~N}_{3} \mathrm{O}_{4}[\mathrm{M}+\mathrm{H}]^{+}:$444.1541, found: 444.1538; $[\alpha]_{D}^{26}=+21.5$ (c = 1.00, $\mathrm{CHCl}_{3}, 97 \%$ ee sample); HPLC analysis $($ CHIRALPAK ID $(\phi=0.46 \mathrm{~cm} \times 25 \mathrm{~cm}), 2$-propanol $/ n$-hexane $=1 / 2$, flow rate $=1.0 \mathrm{~mL} / \mathrm{min}$, detection at $254 \mathrm{~nm}$, tR $=$ $22.3 \min$ (major), $28.3 \min$ (minor)):
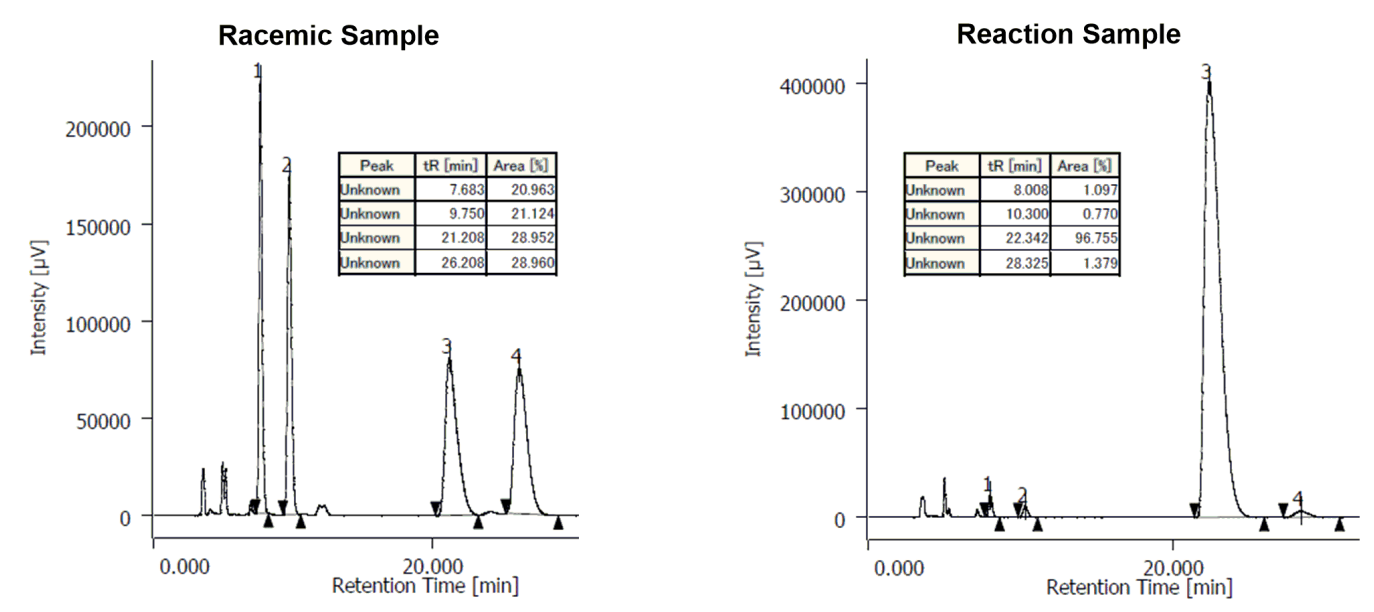

tert-Butyl ((1R,2R)-2-(2,3-dihydro-1H-pyrrolo[2,3-b]pyridine-1-carbonyl)-2,3,3,3-tetrafluoro-1-(furan-3-yl)propyl)-

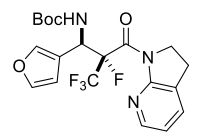
carbamate $(6 \mathrm{p})$.

The reaction was performed according to procedure $\mathrm{B}$ using $\left[\mathrm{Cu}\left(\mathrm{CH}_{3} \mathrm{CN}\right)_{4}\right] \mathrm{PF}_{6}(1.7 \mathrm{mg}, 4.5 \mu \mathrm{mol}$, $1.5 \mathrm{~mol} \%)$, (R)-(-)-2,2'-bis[di(3,5-diisopropyl-4-dimethylaminophenyl)phosphine]-6,6'-dimethoxy-1,1'-biphenyl (DIPAMeO-Biphep) (5.6 mg, $5.1 \mu \mathrm{mol}, 1.7 \mathrm{~mol} \%$ ), anhydrous THF (0.2 mL, $1.2 \mathrm{M})$, 1-(2,3-dihydro-1H-pyrrolo[2,3-b]pyridin-1-yl)-2,3,3,3-tetrafluoropropan-1-one ( $r a c-1 b) ~(74.5 \mathrm{mg}, 0.3 \mathrm{mmol}, 1.0$ equiv.), N-Boc imine 2p (117 mg, 0.6 mmol, 2.0 equiv.), and Barton's base (0.1 M in THF, $45 \mu \mathrm{L}, 4.5 \mu \mathrm{mol}, 1.5 \mathrm{~mol} \%) .125 \mathrm{mg}$ (94\%) of the desired pure product $6 \mathbf{p}$ were obtained after purification by flash column chromatography (hexane/ethyl acetate, 3:2).

White solid, m. p.: 133-135 ${ }^{\circ} \mathrm{C}$; ${ }^{1} \mathrm{H}$ NMR (400 MHz, $\left.343 \mathrm{~K}, \mathrm{DMSO}-d_{6}\right): \delta=8.18-8.16(\mathrm{~m}, 1 \mathrm{H}), 7.66-7.64(\mathrm{~m}, 2 \mathrm{H}), 7.60(\mathrm{brs}$, $1 \mathrm{H}), 7.54(\mathrm{t}, J=1.7 \mathrm{~Hz}, 1 \mathrm{H}), 7.06(\mathrm{dd}, J=7.4,5.0 \mathrm{~Hz}, 1 \mathrm{H}), 6.60(\mathrm{~s}, 1 \mathrm{H}), 6.09(\mathrm{brdd}, J=24.9,9.9 \mathrm{~Hz}, 1 \mathrm{H}), 4.02-3.94(\mathrm{~m}, 1 \mathrm{H})$, 3.90-3.83 (m, 1H), 3.04-2.88 (m, 2H), 1.39 ppm (s, 9H); ${ }^{13} \mathrm{C}$ NMR (125 MHz, $\left.343 \mathrm{~K}, \mathrm{DMSO}-d_{6}\right): \delta=161.1(\mathrm{~d}, J=21.8 \mathrm{~Hz})$, 155.3, 154.3 (br), 145.7, 143.0, 141.1, 133.3, 125.8, 121.5 (qd, $J=287.4,30.0 \mathrm{~Hz}), 119.7,119.6,110.1,96.2$ (dq, $J=218.8,26.3$ $\mathrm{Hz}), 78.6,48.2(\mathrm{brd}, J=18.2 \mathrm{~Hz}), 47.5(\mathrm{~d}, J=11.6 \mathrm{~Hz}), 27.8,25.2 \mathrm{ppm} ;{ }^{19} \mathrm{~F}$ NMR $\left(376 \mathrm{MHz}, 343 \mathrm{~K}, \mathrm{DMSO}-d_{6}\right): \delta=-70.8(\mathrm{~s}$, 

3F), -177.3 ppm (brs, 1F); IR (film): $\widetilde{v}=3330$ (br), 2980, 2928, 1709, 1682, 1596, 1507, 1420, 1393, 1368, 1345, 1309, 1275, 1248, 1207, 1165, 1007, 875, $767 \mathrm{~cm}^{-1}$; HRMS (ESI): m/z calculated for $\mathrm{C}_{20} \mathrm{H}_{22} \mathrm{~F}_{4} \mathrm{~N}_{3} \mathrm{O}_{4}[\mathrm{M}+\mathrm{H}]^{+}:$444.1541, found: 444.1539; $[\alpha]_{D}^{26}=+46.0\left(c=0.85, \mathrm{CHCl}_{3}, 94 \%\right.$ ee sample); HPLC analysis (CHIRALPAK ID $(\phi=0.46 \mathrm{~cm} \times 25 \mathrm{~cm})$, 2-propanol $/ n$-hexane $=1 / 2$, flow rate $=1.0 \mathrm{~mL} / \mathrm{min}$, detection at $254 \mathrm{~nm}, \mathrm{t}_{\mathrm{R}}=20.6 \mathrm{~min}$ (major), $26.7 \mathrm{~min}(\mathrm{minor})$ ):
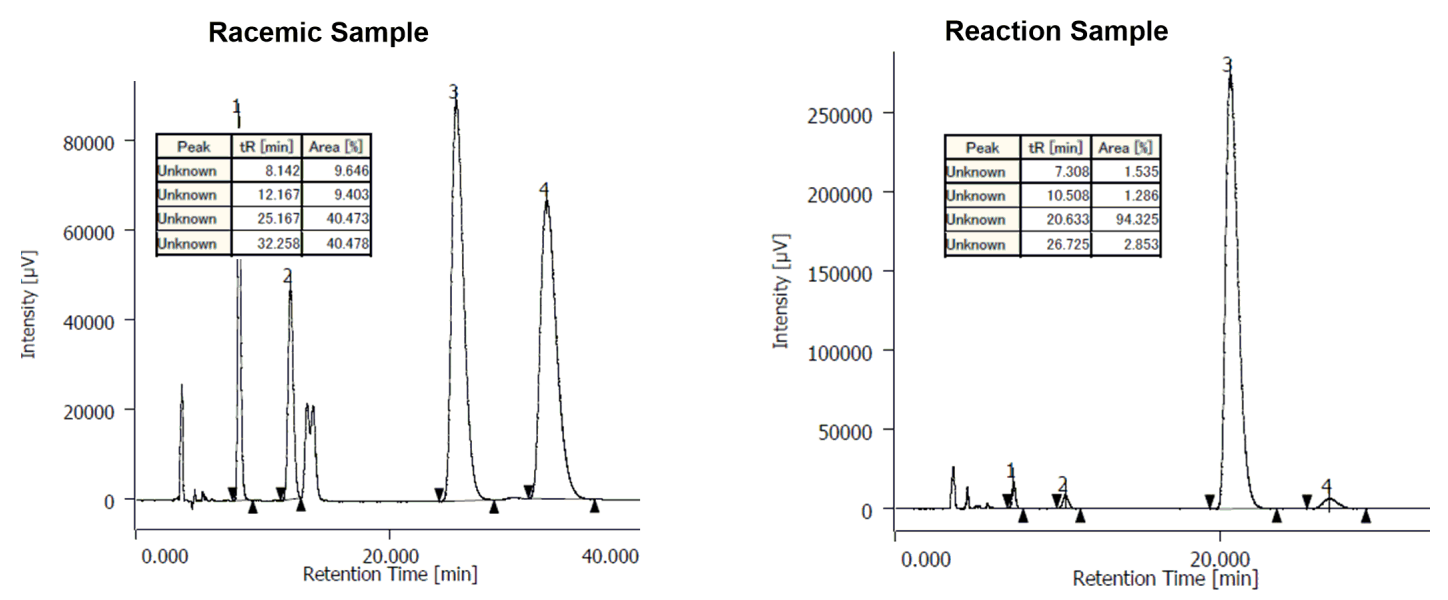

tert-Butyl ((1R,2R)-2-(2,3-dihydro-1H-pyrrolo[2,3-b]pyridine-1-carbonyl)-2,3,3,3-tetrafluoro-1-(thiophen-2-yl)-

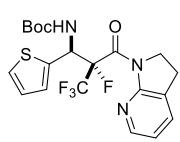
propyl)carbamate $(6 q)$.

The reaction was performed according to procedure B using N-Boc imine $2 \mathbf{q}(42.3 \mathrm{mg}$ ) to afford $41.9 \mathrm{mg}$ $(91 \%)$ of the desired pure product $\mathbf{6 q}$ after purification by flash column chromatography (hexane/ethyl acetate, 3:2).

White solid, m. p.: 140-141 ${ }^{\circ} \mathrm{C}$; ${ }^{1} \mathrm{H}$ NMR (400 MHz, $343 \mathrm{~K}$, DMSO-d6): $\delta=8.17-8.15(\mathrm{~m}, 1 \mathrm{H}), 7.87$ (brs, $\left.1 \mathrm{H}\right), 7.65-7.62$ (m, $1 \mathrm{H}), 7.44(\mathrm{dd}, J=5.1,1.2 \mathrm{~Hz}, 1 \mathrm{H}), 7.22(\mathrm{~d}, J=3.0 \mathrm{~Hz}, 1 \mathrm{H}), 7.06(\mathrm{dd}, J=7.4,5.0 \mathrm{~Hz}, 1 \mathrm{H}), 6.79(\mathrm{dd}, J=5.1,3.6 \mathrm{~Hz}, 1 \mathrm{H}), 6.37$ (brd, $J=16.9 \mathrm{~Hz}, 1 \mathrm{H}), 3.97-3.89(\mathrm{~m}, 1 \mathrm{H}), 3.80-3.72(\mathrm{~m}, 1 \mathrm{H}), 3.00-2.83(\mathrm{~m}, 2 \mathrm{H}), 1.39 \mathrm{ppm}(\mathrm{s}, 9 \mathrm{H}) ;{ }^{13} \mathrm{C} \mathrm{NMR}(125 \mathrm{MHz}, 343$ K, DMSO-d $): \delta=160.8(\mathrm{~d}, J=21.7 \mathrm{~Hz}), 155.0,154.3(\mathrm{br}), 145.6,136.9,133.4,127.3,126.5,126.0,125.8,121.4$ (qd, $J=288.1$, $30.1 \mathrm{~Hz}), 119.7,96.3(\mathrm{dq}, J=217.4,27.0 \mathrm{~Hz}), 78.8,51.2(\mathrm{brd}, J=13.9 \mathrm{~Hz}), 47.6(\mathrm{~d}, J=10.5 \mathrm{~Hz}), 27.8,25.1 \mathrm{ppm} ;{ }^{19} \mathrm{~F} \mathrm{NMR}$ (376 MHz, $\left.343 \mathrm{~K}, \mathrm{DMSO}-d_{6}\right): \delta=-70.6$ (s, 3F), -176.2 ppm (brs, 1F); IR (film): $\widetilde{v}=3337$ (br), 2980, 2932, 1715, 1682, 1506, $1420,1392,1368,1345,1304,1244,1206,1164,1002,760 \mathrm{~cm}^{-1}$; HRMS (ESI): $\mathrm{m} / z$ calculated for $\mathrm{C}_{20} \mathrm{H}_{22} \mathrm{~F}_{4} \mathrm{~N}_{3} \mathrm{O}_{3} S$ [M+H] $]^{+}$ 460.1313, found: 460.1308; $[\alpha]_{D}^{26}=+65.7\left(c=0.80, \mathrm{CHCl}_{3}, 97 \%\right.$ ee sample); HPLC analysis (CHIRALPAK ID $(\phi=0.46 \mathrm{~cm}$ x $25 \mathrm{~cm}$ ), 2-propanol $/ n$-hexane $=1 / 2$, flow rate $=1.0 \mathrm{~mL} / \mathrm{min}$, detection at $254 \mathrm{~nm}, \mathrm{t}_{\mathrm{R}}=22.4 \mathrm{~min}(\mathrm{major}), 32.2 \mathrm{~min}$ (minor)): 

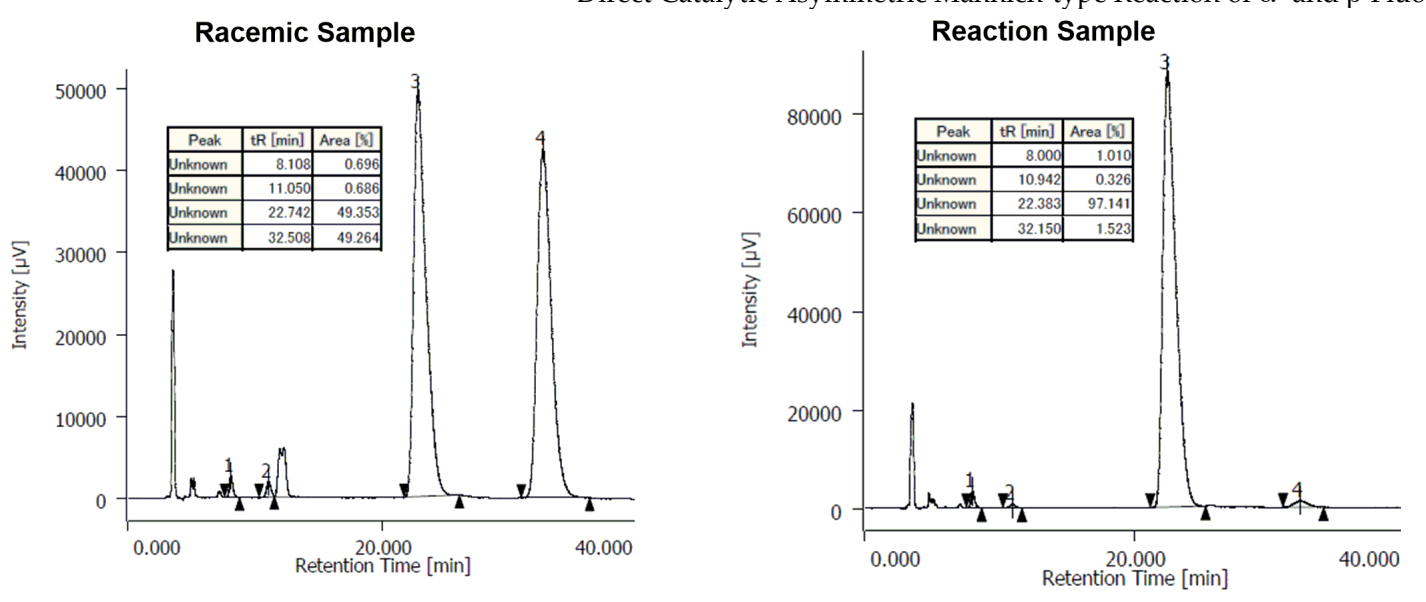

4-5. Direct catalytic asymmetric Mannich-type reaction of $\alpha-\mathrm{CF}_{2} \mathrm{CF}_{3}$ amide $1 \mathrm{c}$ and $\alpha-\mathrm{CF}_{2} \mathrm{CF}_{2} \mathrm{Br}_{\text {amide }} 1 \mathrm{~d}$.

Procedure C:

A flame-dried $20 \mathrm{~mL}$ test tube equipped with a magnetic stirring bar and a 3-way glass stopcock was charged with $\left[\mathrm{Cu}\left(\mathrm{CH}_{3} \mathrm{CN}\right)_{4}\right] \mathrm{PF}_{6}(7.5 \mathrm{mg}, 0.02 \mathrm{mmol}, 20 \mathrm{~mol} \%)$ and $(R)-(+)-5,5$ '-bis[di(3,5-xylyl)phosphino]-4,4'-bi-1,3-benzodioxole (DM-SEGPHOS) (15.9 mg, $0.022 \mathrm{mmol}, 22 \mathrm{~mol} \%)$ in a glove box under Ar atmosphere. Afterwards, the test tube was removed from the glove box and evacuated for $15 \mathrm{~min}$. Then, it was backfilled with Ar and anhydrous THF $(0.10 \mathrm{~mL}$, $0.3 \mathrm{M}$ ) was added at room temperature. The resulting clear colorless solution was stirred for $1 \mathrm{~h}$ before 1-(2,3-dihydro-1H-pyrrolo[2,3-b]pyridin-1-yl)-3,3,4,4,4-pentafluorobutan-1-one (1c) (28.0 mg, $0.1 \mathrm{mmol}, 1.0$ equiv.) and the corresponding $N$-Boc or $N-C b z$ imine $(\mathbf{2 a - 2 c}, \mathbf{2 e}, \mathbf{2} \mathbf{g}, \mathbf{2 k}, \mathbf{2 m}, \mathbf{2 0}, \mathbf{2 s})$ (0.2 mmol, 2.0 equiv.) were added. The reaction mixture was then cooled to $0{ }^{\circ} \mathrm{C}$ and finally Barton's base (a $0.1 \mathrm{M}$ stock solution of Barton's base was prepared by diluting the commercial pure amine with the appropriate amount of freshly dried anhydrous THF and used for a maximum time period of 2 weeks before it was discarded; $0.1 \mathrm{M}$ in THF, $0.2 \mathrm{~mL}, 0.02 \mathrm{mmol}, 20 \mathrm{~mol} \%$ ) was added. The reaction mixture was stirred at $0^{\circ} \mathrm{C}$ until the complete consumption of the amide starting material was observed by TLC analysis $(<24 \mathrm{~h})$. The diastereomeric ratio and the enantiomeric excess of the target material were determined by HPLC analysis of an aliquot, which was taken from the reaction mixture and filtered through a short pad of silica; the diastereomeric ratio is reported if it is worse than 20:1. Then, the reaction mixture was loaded directly onto a silica gel column and eluted with a suitable solution of $n$-hexane and ethyl acetate. Evaporation of solvent afforded the pure product.

Note that the NMR spectra of the Mannich products were recorded at elevated temperature (usually $\left.70{ }^{\circ} \mathrm{C}\right)$ in DMSO-d6, as rotamers were observed in $\mathrm{CDCl}_{3}$ at room temperature. ${ }^{10}$

\section{tert-Butyl ((1S,2S)-2-(2,3-dihydro-1H-pyrrolo[2,3-b]pyridine-1-carbonyl)-3,3,4,4,4-pentafluoro-1-phenylbutyl)-}

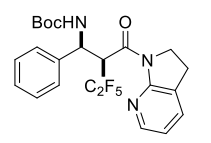
carbamate (12a).

The reaction was performed according to procedure C using N-Boc imine $2 \mathbf{a}(41.0 \mathrm{mg})$ to afford $42.0 \mathrm{mg}$ $(87 \%)$ of the desired pure product 12a after purification by flash column chromatography (hexane/ethyl acetate, $2: 1)$.

White solid, m. p.: $172-174{ }^{\circ} \mathrm{C} ;{ }^{1} \mathrm{H}$ NMR (400 MHz, $\left.343 \mathrm{~K}, \mathrm{DMSO}-d_{6}\right): \delta=8.16-8.13(\mathrm{~m}, 1 \mathrm{H}), 7.58(\mathrm{brs}, 1 \mathrm{H}), 7.56(\mathrm{ddd}, J=$ 

7.4, 2.8, 1.3 Hz, 1H), 7.25-7.22 (m, 2H), 7.11-7.07 (m, 1H), 7.05-7.01 (m, 3H), $6.50(\mathrm{dt}, J=19.7,10.3 \mathrm{~Hz}, 1 \mathrm{H}), 5.33(\mathrm{t}, J=$ $10.2 \mathrm{~Hz}, 1 \mathrm{H}), 3.70(\mathrm{ddd}, J=12.1,10.5,7.0 \mathrm{~Hz}, 1 \mathrm{H}), 3.33(\mathrm{ddd}, J=12.1,10.7,5.5 \mathrm{~Hz}, 1 \mathrm{H}), 2.86-2.78(\mathrm{~m}, 1 \mathrm{H}), 2.57-2.51(\mathrm{~m}$, 1H), 1.35 (s, 9H); ${ }^{13} \mathrm{C}$ NMR (125 MHz, $\left.343 \mathrm{~K}, \mathrm{DMSO}-\mathrm{d}_{6}\right): \delta=162.9$ (d, J = 9.2 Hz), 154.0 (br), 153.7, 144.9, 139.1, 134.0, 127.4, 127.1, 127.0, 126.0, 118.7, 118.5 (qt, $J=287.8,36.8 \mathrm{~Hz}), 114.4(\mathrm{~m}), 77.8,53.1$ (br), 48.1 (t, J = 18.6 Hz), 45.4, $27.9,22.8$ ppm; ${ }^{19} \mathrm{~F}$ NMR (376 MHz, $\left.343 \mathrm{~K}, \mathrm{DMSO}-\mathrm{d}_{6}\right): \delta=-81.3(\mathrm{~s}, 3 \mathrm{~F}),-110.0$ (brd, $\left.J=273.5 \mathrm{~Hz}, 1 \mathrm{~F}\right),-114.6 \mathrm{ppm}(\mathrm{dd}, J=273.7$, $18.8 \mathrm{~Hz}, 1 \mathrm{~F})$; IR (film): $\widetilde{v}=3297$ (br), 3032, 3007, 2973, 1711, 1647, 1595, 1524, 1431, 1365, 1278, 1241, 1213, 1193, 1115, 1042, 1022, 861, $772 \mathrm{~cm}^{-1}$; HRMS (ESI): $m / z$ calculated for $\mathrm{C}_{23} \mathrm{H}_{24} \mathrm{~F}_{5} \mathrm{~N}_{3} \mathrm{O}_{3} \mathrm{Na}[\mathrm{M}+\mathrm{Na}]^{+}: 508.1630$, found: $508.1629 ;[\alpha]_{D}^{25}=-66.7$ ( $c=0.30, \mathrm{CHCl}_{3}, 97 \%$ ee sample); HPLC analysis (CHIRALPAK IA $(\phi=0.46 \mathrm{~cm} \times 25 \mathrm{~cm}), 2$-propanol $/ n$-hexane $=1 / 20$, flow rate $=1.0 \mathrm{~mL} / \mathrm{min}$, detection at $254 \mathrm{~nm}, \mathrm{t}_{\mathrm{R}}=17.6 \mathrm{~min}$ (major), $19.4 \mathrm{~min}$ (minor)):
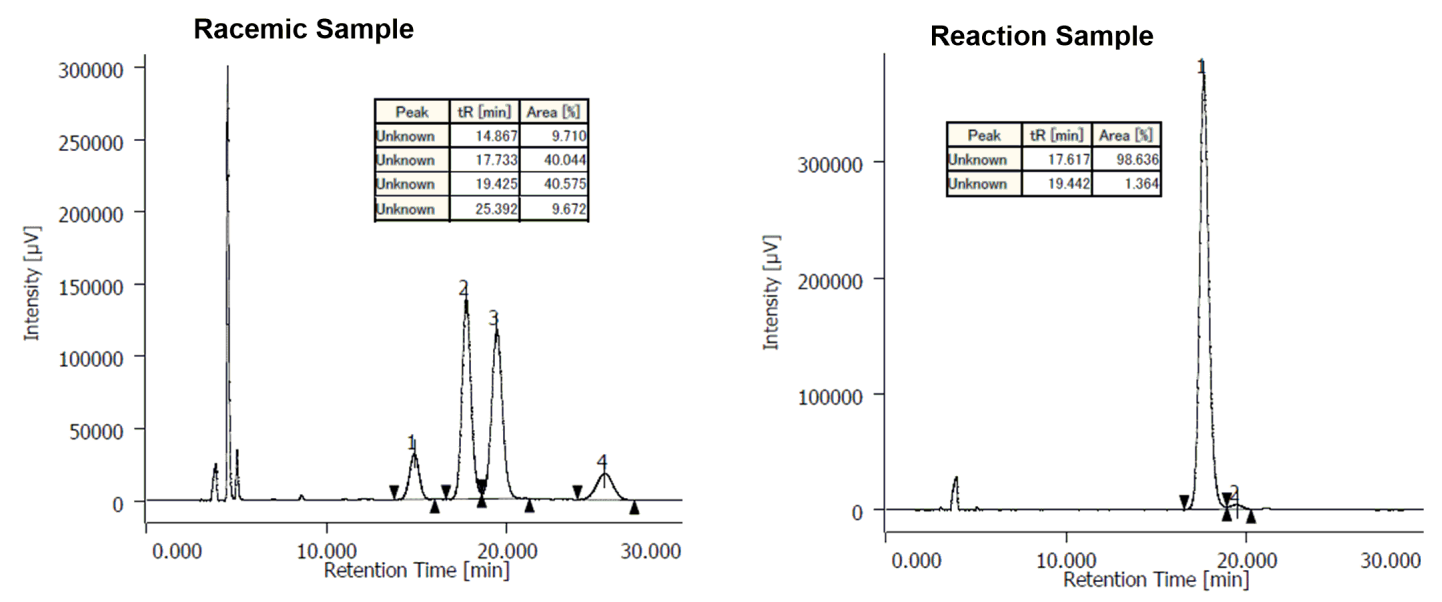

Benzyl ((1S,2S)-2-(2,3-dihydro-1H-pyrrolo[2,3-b]pyridine-1-carbonyl)-3,3,4,4,4-pentafluoro-1-phenylbutyl)carbamate

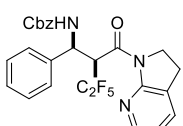
(12b).

The reaction was performed according to procedure $\mathrm{C}$ using $\mathrm{N}-\mathrm{Cbz}$ imine $\mathbf{2 b}(47.9 \mathrm{mg})$ to afford $44.1 \mathrm{mg}$ $(84 \%)$ of the desired pure product $\mathbf{1 2 b}$ after purification by flash column chromatography (hexane/ethyl acetate, $2: 1)$.

White solid, m. p.: 159-160 ${ }^{\circ} \mathrm{C} ;{ }^{1} \mathrm{H}$ NMR (400 MHz, $343 \mathrm{~K}$, DMSO-d6): $\delta=8.16-8.09$ (m, 2H), 7.57-7.55 (m, 1H), 7.34-7.24 $(\mathrm{m}, 7 \mathrm{H}), 7.12-7.08(\mathrm{~m}, 1 \mathrm{H}), 7.06-7.01(\mathrm{~m}, 3 \mathrm{H}), 6.56(\mathrm{dt}, J=20.6,9.9 \mathrm{~Hz}, 1 \mathrm{H}), 5.39(\mathrm{t}, J=10.2 \mathrm{~Hz}, 1 \mathrm{H}), 5.05(\mathrm{~d}, J=12.7 \mathrm{~Hz}$, $1 \mathrm{H}), 4.98(\mathrm{~d}, J=12.7 \mathrm{~Hz}, 1 \mathrm{H}), 3.71(\mathrm{ddd}, J=12.1,10.6,7.0 \mathrm{~Hz}, 1 \mathrm{H}), 3.35(\mathrm{ddd}, J=12.1,10.8,5.5 \mathrm{~Hz}, 1 \mathrm{H}), 2.87-2.79(\mathrm{~m}, 1 \mathrm{H})$, 2.58-2.51 ppm (m, 1H); ${ }^{13} \mathrm{C}$ NMR (125 MHz, $\left.343 \mathrm{~K}, \mathrm{DMSO}-\mathrm{d}_{6}\right): \delta=162.7$ (d, J = 9.2 Hz), 154.7 (br), $153.6,144.9,138.8$, 136.7, 134.0, 127.9 (2C), 127.4 (2C), 127.3, 127.2 (4C), 127.1, 126.0, 118.8, 118.5 (qt, J = 287.9, 36.7 Hz), 114.3 (m), $65.2,53.7$ (br), $48.0(\mathrm{t}, J=18.6 \mathrm{~Hz}), 45.4,22.8$ ppm; ${ }^{19} \mathrm{~F}$ NMR (376 MHz, $\left.343 \mathrm{~K}, \mathrm{DMSO}-d_{6}\right): \delta=-81.3$ (s, 3F), -109.5 (brd, $J=272.7 \mathrm{~Hz}$, 1F), -114.8 ppm (dd, $J=273.9,18.9 \mathrm{~Hz}, 1 \mathrm{~F})$; IR (film): $\widetilde{v}=3320$ (br), 3062, 3032, 2956, 1716, 1653, 1594, 1541, 1425, 1335, 1241, 1218, 1192, 1114, 1040, $772 \mathrm{~cm}^{-1}$; HRMS (ESI): $\mathrm{m} / z$ calculated for $\mathrm{C}_{26} \mathrm{H}_{22} \mathrm{~F}_{5} \mathrm{~N}_{3} \mathrm{O}_{3} \mathrm{Na}[\mathrm{M}+\mathrm{Na}]^{+}: 542.1474$, found: 542.1470; $[\alpha]_{D}^{25}=-84.9\left(c=0.30, \mathrm{CHCl}_{3}, 97 \%\right.$ ee sample); HPLC analysis (CHIRALPAK IF $(\phi=0.46 \mathrm{~cm} \times 25 \mathrm{~cm})$, 2-propanol $/ n$-hexane $=1 / 9$, flow rate $=1.0 \mathrm{~mL} / \mathrm{min}$, detection at $254 \mathrm{~nm}, \mathrm{t}_{\mathrm{R}}=30.2 \mathrm{~min}$ (major), $34.0 \mathrm{~min}(\mathrm{minor})$ ): 

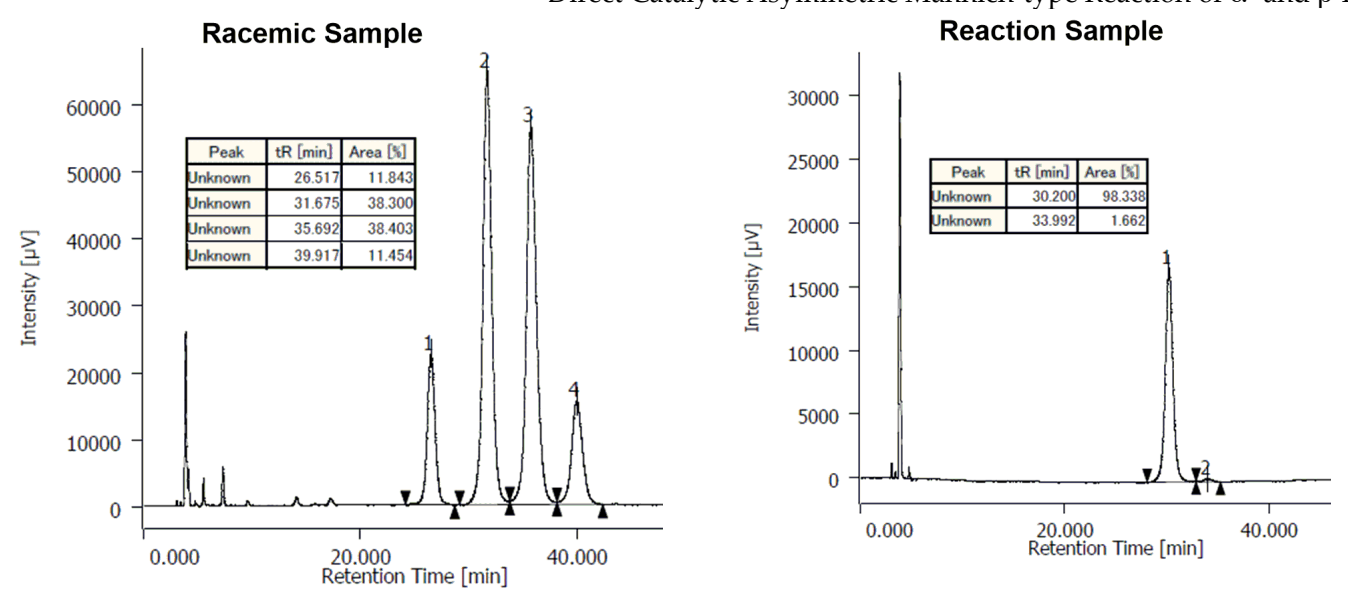

tert-Butyl ((1S,2S)-2-(2,3-dihydro-1H-pyrrolo[2,3-b]pyridine-1-carbonyl)-3,3,4,4,4-pentafluoro-1-(naphthalen-2-yl)-

\section{BochN ${ }^{\circ}$ butyl)carbamate (12c).}

The reaction was performed according to procedure $C$ using $N$-Boc imine $2 \mathrm{c}(51.1 \mathrm{mg})$ to afford 39.1 mg $(73 \%)$ of the desired pure product $12 \mathrm{c}$ after purification by flash column chromatography (hexane/ethyl acetate, 2:1).

White solid, m. p.: $191-193{ }^{\circ} \mathrm{C}$; ${ }^{1} \mathrm{H}$ NMR $\left(400 \mathrm{MHz}, 343 \mathrm{~K}\right.$, DMSO- $\left.\mathrm{d}_{6}\right): \delta=8.20(\mathrm{~d}, J=5.0 \mathrm{~Hz}, 1 \mathrm{H}), 7.78-7.72(\mathrm{~m}, 3 \mathrm{H})$, 7.58-7.54 (m, 2H), 7.43-7.37 (m, 4H), $7.01(\mathrm{dd}, J=7.4,5.1 \mathrm{~Hz}, 1 \mathrm{H}), 6.61(\mathrm{dt}, J=20.2,10.1 \mathrm{~Hz}, 1 \mathrm{H}), 5.52(\mathrm{brt}, J=10.1 \mathrm{~Hz}$, 1H), $3.60(\mathrm{ddd}, J=12.1,10.5,7.1 \mathrm{~Hz}, 1 \mathrm{H}), 3.18(\mathrm{ddd}, J=12.0,10.7,5.3 \mathrm{~Hz}, 1 \mathrm{H}), 2.65(\mathrm{ddd}, J=16.6,10.6,5.4 \mathrm{~Hz}, 1 \mathrm{H}), 2.04$ $(\mathrm{ddd}, J=17.1,10.1,6.9 \mathrm{~Hz}, 1 \mathrm{H}), 1.35 \mathrm{ppm}(\mathrm{s}, 9 \mathrm{H}) ;{ }^{13} \mathrm{C}$ NMR $\left(125 \mathrm{MHz}, 343 \mathrm{~K}, \mathrm{DMSO}-d_{6}\right): \delta=162.0(\mathrm{~d}, J=9.2 \mathrm{~Hz}), 154.0$ (br), 153.6, 144.9, 136.6, 133.9, 131.9(4), 131.9, 127.1, 126.9, 126.6, 126.3, 125.9, 125.6 (2C), 125.5, 118.8, 118.5 (qt, $J=288.2$, $36.7 \mathrm{~Hz}), 114.4(\mathrm{~m}), 77.9,53.2(\mathrm{br}), 48.1(\mathrm{t}, J=18.6 \mathrm{~Hz}), 45.3,27.8,22.6$ ppm; ${ }^{19} \mathrm{~F}$ NMR $\left(376 \mathrm{MHz}, 343 \mathrm{~K}, \mathrm{DMSO}-d_{6}\right): \delta=$ $-81.3(\mathrm{~s}, 3 \mathrm{~F}),-110.0(\mathrm{brd}, J=272.5 \mathrm{~Hz}, 1 \mathrm{~F}),-114.6 \mathrm{ppm}(\mathrm{dd}, J=274.1,19.2 \mathrm{~Hz}, 1 \mathrm{~F}) ; \mathrm{IR}($ film): $\widetilde{v}=3295,3038,2970,2928$, 1712, 1645, 1594, 1530, 1429, 1411, 1366, 1336, 1278, 1241, 1212, 1192, 1117, 1042, 1025, 862, $793 \mathrm{~cm}^{-1}$; HRMS (ESI): m/z calculated for $\mathrm{C}_{27} \mathrm{H}_{26} \mathrm{~F}_{5} \mathrm{~N}_{3} \mathrm{O}_{3} \mathrm{Na}[\mathrm{M}+\mathrm{Na}]^{+}: 558.1787$, found: 558.1785; $[\alpha]_{D}^{25}=-158.2\left(c=1.15, \mathrm{CHCl}_{3}, 95 \%\right.$ ee sample); HPLC analysis (CHIRALPAK IF $(\phi=0.46 \mathrm{~cm} \times 25 \mathrm{~cm}), 2$-propanol $/ n$-hexane $=1 / 13$, flow rate $=1.0 \mathrm{~mL} / \mathrm{min}$, detection at $254 \mathrm{~nm}, \mathrm{t}_{\mathrm{R}}=17.7 \mathrm{~min}$ (major), $25.8 \mathrm{~min}$ (minor)):
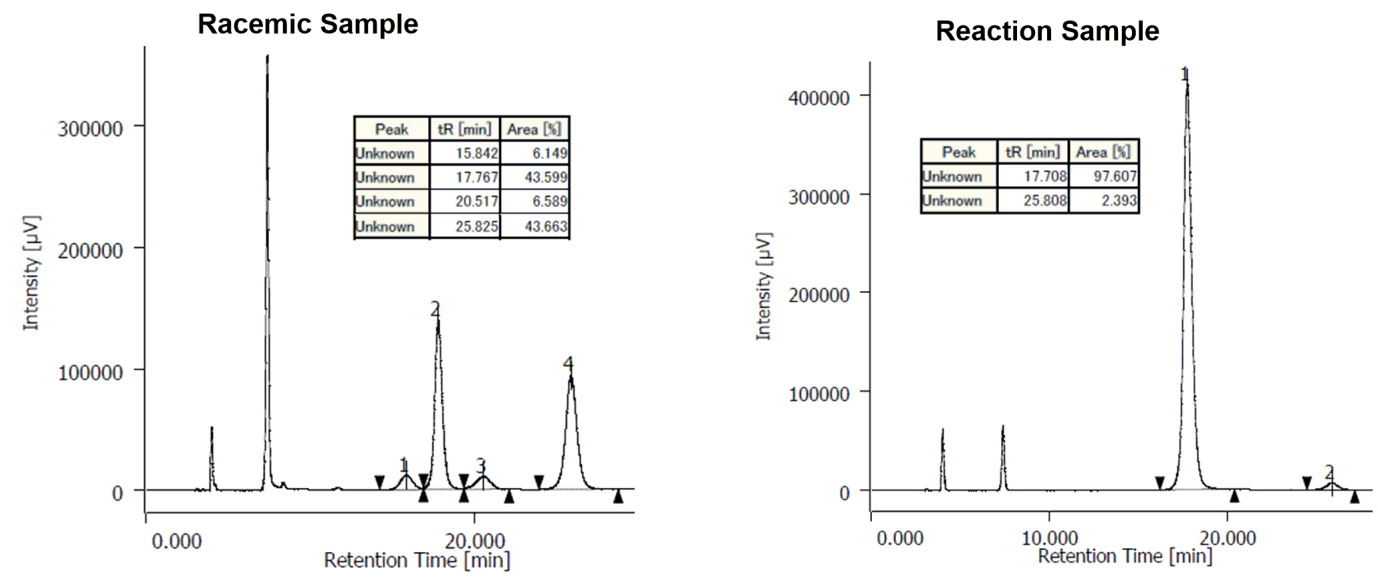

tert-Butyl ((1S,2S)-2-(2,3-dihydro-1H-pyrrolo[2,3-b]pyridine-1-carbonyl)-3,3,4,4,4-pentafluoro-1-(para-tolyl)butyl)-

BochN $O$ carbamate (12e).

The reaction was performed according to procedure C using N-Boc imine $2 \mathrm{e}(43.9 \mathrm{mg})$ to afford $42.3 \mathrm{mg}$ $(84 \%)$ of the desired pure product 12e after purification by flash column chromatography (hexane/ethyl acetate, 2:1).

White solid, m. p.: $167-170{ }^{\circ} \mathrm{C} ;{ }^{1} \mathrm{H}$ NMR (400 MHz, $\left.343 \mathrm{~K}, \mathrm{DMSO}-d_{6}\right): \delta=8.17-8.14$ (m, 1H), 7.58 (ddd, J = 7.4, $2.8,1.3 \mathrm{~Hz}$, 1H), 7.55 (brs, 1H), 7.13-7.10 (m, 2H), $7.04(\mathrm{dd}, J=7.4,5.0 \mathrm{~Hz}, 1 \mathrm{H}), 6.84(\mathrm{~d}, J=7.8 \mathrm{~Hz}, 2 \mathrm{H}), 6.47$ (dt, $J=20.8,9.8 \mathrm{~Hz}, 1 \mathrm{H})$, $5.30(\mathrm{t}, J=10.2 \mathrm{~Hz}, 1 \mathrm{H}), 3.71(\mathrm{ddd}, J=12.1,10.5,7.1 \mathrm{~Hz}, 1 \mathrm{H}), 3.40(\mathrm{ddd}, J=12.1,10.7,5.4 \mathrm{~Hz}, 1 \mathrm{H}), 2.88-2.80(\mathrm{~m}, 1 \mathrm{H})$, 2.59-2.52 (m, 1H), $2.16(\mathrm{~s}, 3 \mathrm{H}), 1.37 \mathrm{ppm}(\mathrm{s}, 9 \mathrm{H}) ;{ }^{13} \mathrm{C}$ NMR (125 MHz, $\left.343 \mathrm{~K}, \mathrm{DMSO}-d_{6}\right): \delta=163.0(\mathrm{~d}, J=9.1 \mathrm{~Hz}), 153.9$ (br), 153.7, 144.9, 136.2(3), 136.2, 133.9, 127.6, 127.3, 126.0, 118.7, 118.5 (qt, J = 287.6, 36.9 Hz), 114.4 (m), $77.7,52.8$ (br), $48.2(\mathrm{t}, J=18.6 \mathrm{~Hz}), 45.4,27.9,22.8,20.1 \mathrm{ppm}$; ${ }^{19} \mathrm{~F}$ NMR $\left(376 \mathrm{MHz}, 343 \mathrm{~K}, \mathrm{DMSO}-d_{6}\right): \delta=-81.3(\mathrm{~s}, 3 \mathrm{~F}),-110.0(\mathrm{brd}, J=$ 275.1 Hz, 1F), -114.5 ppm (dd, $J=273.4,18.8 \mathrm{~Hz}, 1 \mathrm{~F})$; IR (film): $\widetilde{v}=3300,3032,3007,2973,2918,1712,1647,1595,1530$, $1429,1366,1277,1241,1212,1193,1116,1042,1024,772 \mathrm{~cm}^{-1}$; HRMS (ESI): $\mathrm{m} / z$ calculated for $\mathrm{C}_{24} \mathrm{H}_{26} \mathrm{~F}_{5} \mathrm{~N}_{3} \mathrm{O}_{3} \mathrm{Na}_{[\mathrm{M}+\mathrm{Na}}{ }^{+}$: 522.1787, found: 522.1785; $[\alpha]_{D}^{25}=-124.2\left(c=0.25, \mathrm{CHCl}_{3}, 93 \%\right.$ ee sample); HPLC analysis (CHIRALPAK IA $(\phi=0.46$ $\mathrm{cm} \times 25 \mathrm{~cm}$ ), 2-propanol $/ n$-hexane $=1 / 20$, flow rate $=1.0 \mathrm{~mL} / \mathrm{min}$, detection at $254 \mathrm{~nm}, \mathrm{t}=16.3 \mathrm{~min}(\mathrm{major}), 20.3 \mathrm{~min}$ (minor)):
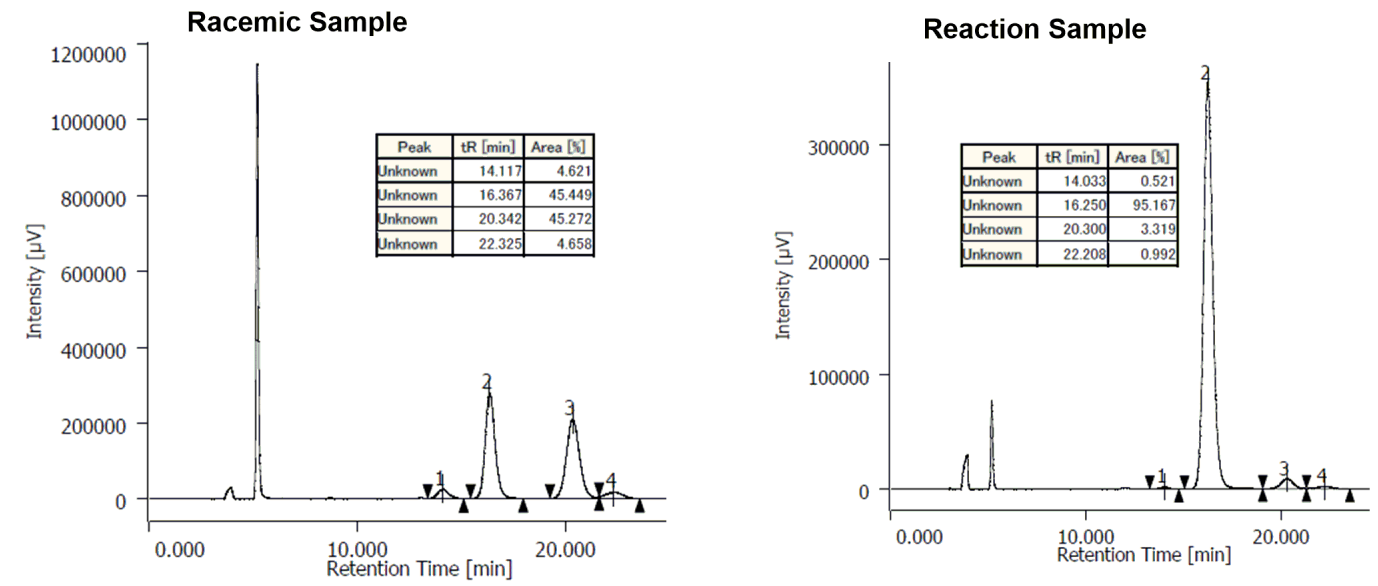

tert-Butyl ((1S,2S)-2-(2,3-dihydro-1H-pyrrolo[2,3-b]pyridine-1-carbonyl)-3,3,4,4,4-pentafluoro-1-(3-methoxyphenyl)-

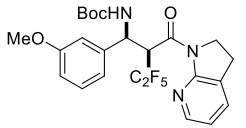
butyl)carbamate (12g).

The reaction was performed according to procedure C using N-Boc imine $2 \mathrm{~g}(47.1 \mathrm{mg})$ to afford 41.9 mg $(82 \%)$ of the desired pure product $\mathbf{1 2} \mathrm{g}$ after purification by flash column chromatography (hexane/ethyl acetate, $2: 1)$.

White solid, m. p.: $162-164{ }^{\circ} \mathrm{C} ;{ }^{1} \mathrm{H}$ NMR (400 MHz, $\left.343 \mathrm{~K}, \mathrm{DMSO}-d_{6}\right): \delta=8.16-8.14(\mathrm{~m}, 1 \mathrm{H}), 7.59-7.56$ (m, $\left.2 \mathrm{H}\right), 7.02(\mathrm{dd}$, $J=7.4,5.1 \mathrm{~Hz}, 1 \mathrm{H}), 6.98(\mathrm{t}, J=7.9 \mathrm{~Hz}, 1 \mathrm{H}), 6.91(\mathrm{dd}, J=2.1,1.9 \mathrm{~Hz}, 1 \mathrm{H}), 6.77-6.74(\mathrm{~m}, 1 \mathrm{H}), 6.65(\mathrm{ddd}, J=8.2,2.6,0.9 \mathrm{~Hz}$, $1 \mathrm{H}), 6.49(\mathrm{dt}, J=21.1,9.6 \mathrm{~Hz}, 1 \mathrm{H}), 5.31(\mathrm{t}, J=10.3 \mathrm{~Hz}, 1 \mathrm{H}), 3.72(\mathrm{ddd}, J=12.1,10.5,7.1 \mathrm{~Hz}, 1 \mathrm{H}), 3.44(\mathrm{~s}, 3 \mathrm{H}), 3.36(\mathrm{ddd}, J=$ $12.1,10.7,5.4 \mathrm{~Hz}, 1 \mathrm{H}), 2.88-2.80(\mathrm{~m}, 1 \mathrm{H}), 2.61-2.52(\mathrm{~m}, 1 \mathrm{H}), 1.36 \mathrm{ppm}(\mathrm{s}, 9 \mathrm{H}) ;{ }^{13} \mathrm{C}$ NMR $\left(125 \mathrm{MHz}, 343 \mathrm{~K}, \mathrm{DMSO}-d_{6}\right): \delta=$ $162.8(\mathrm{~d}, J=9.0 \mathrm{~Hz}), 158.6,153.9(\mathrm{br}), 153.7,144.9,140.6,134.0,128.1,126.0,120.0,118.7,118.5$ (qt, $J=287.7,36.8 \mathrm{~Hz})$, $114.4(\mathrm{~m}), 113.5,112.3,77.8,54.4,53.1(\mathrm{br}), 48.1(\mathrm{t}, J=18.6 \mathrm{~Hz}), 45.5,27.9,22.8$ ppm; ${ }^{19} \mathrm{~F} \mathrm{NMR}(376 \mathrm{MHz}, 343 \mathrm{~K}$, DMSO-d $): \delta=-81.3(\mathrm{~s}, 3 \mathrm{~F}),-110.3$ (brd, $J=272.5 \mathrm{~Hz}, 1 \mathrm{~F}),-114.5 \mathrm{ppm}(\mathrm{dd}, J=274.2,18.7 \mathrm{~Hz}, 1 \mathrm{~F})$; IR (film): $\widetilde{v}=3344,3059$, 

3011, 2970, 1703, 1667, 1595, 1507, 1490, 1424, 1242, 1218, 1191, 1117, 1043, $772 \mathrm{~cm}^{-1}$; HRMS (ESI): m/z calculated for $\mathrm{C}_{24} \mathrm{H}_{26} \mathrm{~F}_{5} \mathrm{~N}_{3} \mathrm{O}_{4} \mathrm{Na}[\mathrm{M}+\mathrm{Na}]^{+}: 538.1736$, found: $538.1738 ;[\alpha]_{D}^{25}=-97.6\left(c=0.55, \mathrm{CHCl}_{3}, 95 \%\right.$ ee sample); HPLC analysis $($ CHIRALPAK IA $(\phi=0.46 \mathrm{~cm} \times 25 \mathrm{~cm}), 2$-propanol $/ n$-hexane $=1 / 20$, flow rate $=1.0 \mathrm{~mL} / \mathrm{min}$, detection at $254 \mathrm{~nm}$, tR $=$ $22.3 \mathrm{~min}$ (major), $35.8 \mathrm{~min}$ (minor)):
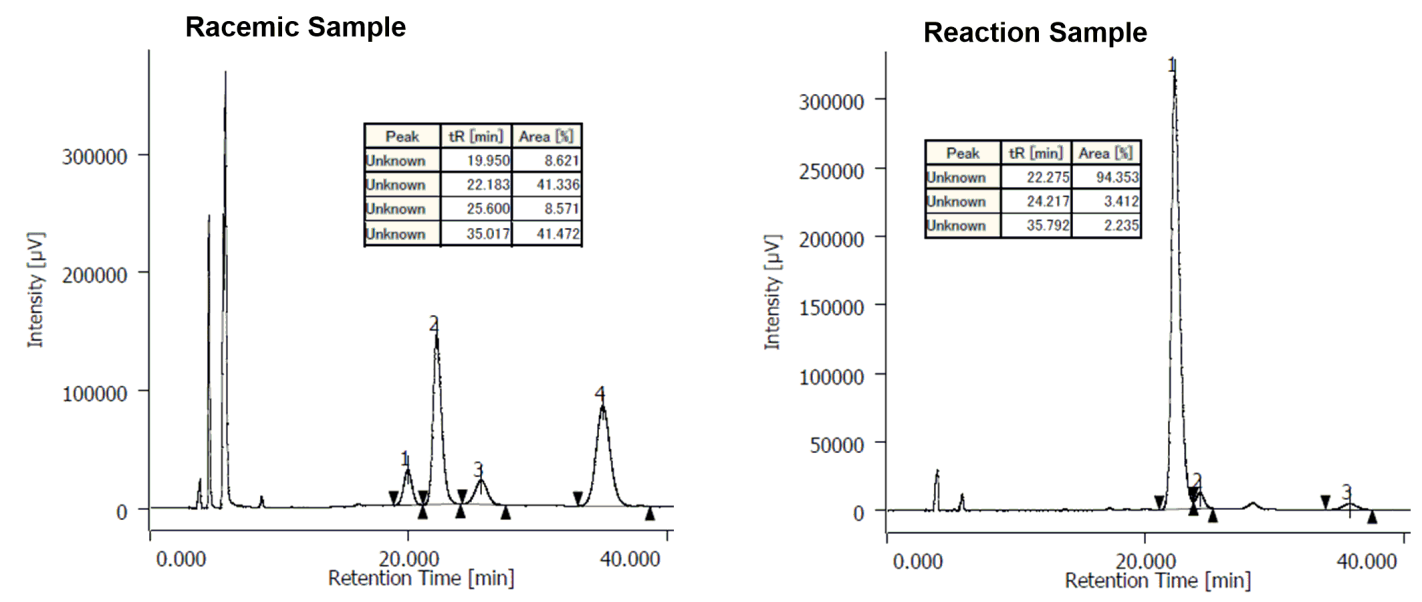

tert-Butyl ((1S,2S)-2-(2,3-dihydro-1H-pyrrolo[2,3-b]pyridine-1-carbonyl)-3,3,4,4,4-pentafluoro-1-(4-fluorophenyl)-

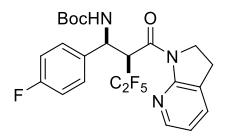
butyl)carbamate (12k).

The reaction was performed according to procedure C using N-Boc imine $2 \mathbf{k}(44.7 \mathrm{mg})$ to afford 40.4 mg $(79 \%)$ of the desired pure product 12k after purification by flash column chromatography (hexane/ethyl acetate, $3: 1)$.

White solid, m. p.: $165-167^{\circ} \mathrm{C}$; ${ }^{1} \mathrm{H}$ NMR (400 MHz, $343 \mathrm{~K}$, DMSO-d6): $\delta=8.15-8.13$ (m, 1H), 7.64 (brs, $\left.1 \mathrm{H}\right), 7.59(\mathrm{ddd}, J=$ 7.4, 2.7, $1.3 \mathrm{~Hz}, 1 \mathrm{H}), 7.31-7.26(\mathrm{~m}, 2 \mathrm{H}), 7.04(\mathrm{dd}, J=7.4,5.1 \mathrm{~Hz}, 1 \mathrm{H}), 6.87-6.81(\mathrm{~m}, 2 \mathrm{H}), 6.50(\mathrm{dt}, J=20.7,9.8 \mathrm{~Hz}, 1 \mathrm{H}), 5.33$ $(\mathrm{t}, J=10.2 \mathrm{~Hz}, 1 \mathrm{H}), 3.74(\mathrm{ddd}, J=12.2,10.6,6.8 \mathrm{~Hz}, 1 \mathrm{H}), 3.42(\mathrm{ddd}, J=12.1,10.7,5.6 \mathrm{~Hz}, 1 \mathrm{H}), 2.91-2.82(\mathrm{~m}, 1 \mathrm{H}), 2.66-2.57$ (m, 1H), 1.35 ppm (s, 9H); ${ }^{13} \mathrm{C}$ NMR (125 MHz, $\left.343 \mathrm{~K}, \mathrm{DMSO}-\mathrm{d}_{6}\right): \delta=162.8(\mathrm{~d}, J=9.0 \mathrm{~Hz}), 161.1(\mathrm{~d}, J=244.1 \mathrm{~Hz}), 153.9$ (br), 153.6, 144.9, 135.5, 134.1, $129.5(\mathrm{~d}, J=8.1 \mathrm{~Hz}), 126.0,118.9,118.5(\mathrm{qt}, J=287.7,36.8 \mathrm{~Hz}), 114.3(\mathrm{~m}), 113.8(\mathrm{~d}, J=21.3$ $\mathrm{Hz}), 77.9,52.4$ (br), 48.1 (t, $J=18.6 \mathrm{~Hz}), 45.4,27.8,22.8$ ppm; ${ }^{19} \mathrm{~F}$ NMR $\left(376 \mathrm{MHz}, 343 \mathrm{~K}, \mathrm{DMSO}-d_{6}\right): \delta=-81.3(\mathrm{~s}, 3 \mathrm{~F})$, -110.0 (brd, $J=270.3 \mathrm{~Hz}, 1 \mathrm{~F}),-114.6$ (dd, $J=274.0,19.1 \mathrm{~Hz}, 1 \mathrm{~F}),-114.9$ ppm (s, 1F); IR (film): $\widetilde{v}=3300$ (br), 3004,2973 , 2925, 1712, 1646, 1595, 1509, 1433, 1366, 1278, 1210, 1192, 1116, 1042, 1022, 843, $772 \mathrm{~cm}^{-1}$; HRMS (ESI): m/z calculated for $\mathrm{C}_{23} \mathrm{H}_{23} \mathrm{~F}_{6} \mathrm{~N}_{3} \mathrm{O}_{3} \mathrm{Na}[\mathrm{M}+\mathrm{Na}]^{+}: 526.1536$, found: $526.1531 ;[\alpha]_{D}^{26}=-81.9\left(c=0.40, \mathrm{CHCl}_{3}, 98 \%\right.$ ee sample); HPLC analysis (CHIRALPAK IA-3 $(\phi=0.46 \mathrm{~cm} \times 25 \mathrm{~cm}), 2$-propanol $/ n$-hexane $=1 / 12$, flow rate $=0.5 \mathrm{~mL} / \mathrm{min}$, detection at $254 \mathrm{~nm}$, tR $=$ $21.9 \mathrm{~min}$ (major), $25.5 \mathrm{~min}$ (minor)): 

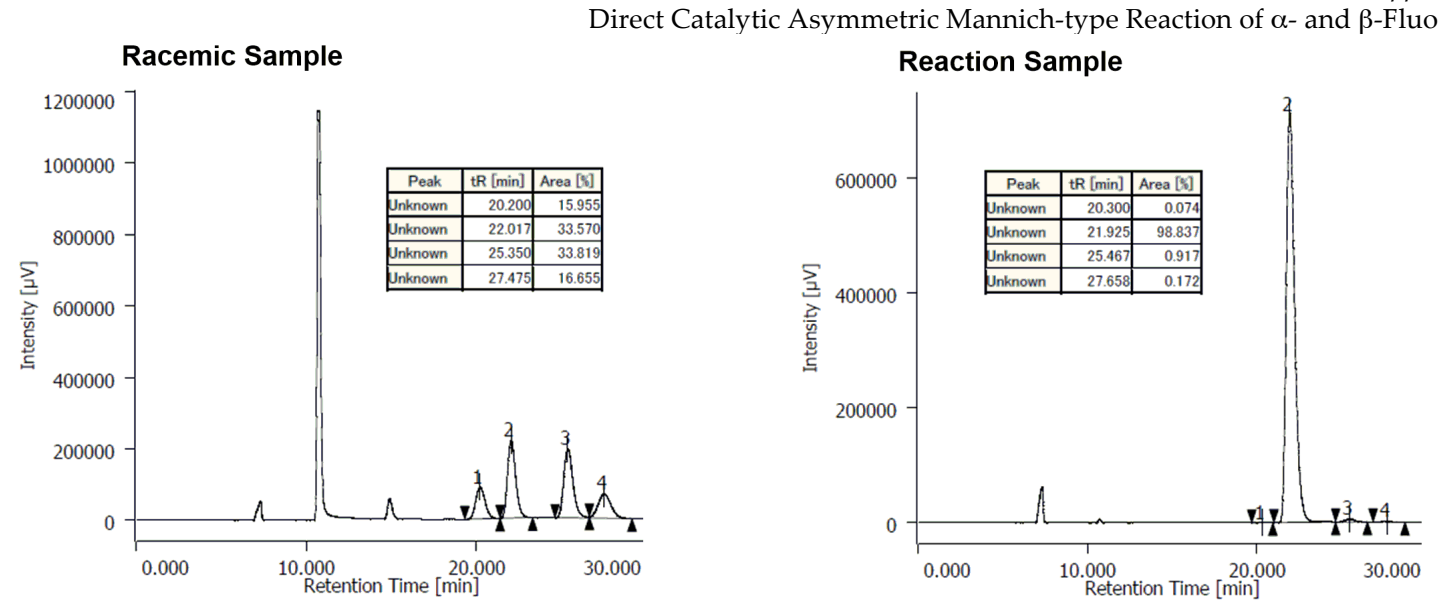

tert-Butyl ((1S,2S)-2-(2,3-dihydro-1H-pyrrolo[2,3-b]pyridine-1-carbonyl)-3,3,4,4,4-pentafluoro-1-(3-nitrophenyl)-

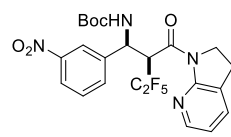

butyl)carbamate $(\mathbf{1 2 m})$.

The reaction was performed according to procedure C using N-Boc imine $2 \mathrm{~m}$ (50.1 $\mathrm{mg})$ to afford 46.0 $\mathrm{mg}(87 \%)$ of the desired product $\mathbf{1 2 m}$ as a $13.5: 1$ mixture of diastereomers after purification by flash column chromatography (hexane/ethyl acetate, 2:1). Analytical data for the major diastereomer are presented below:

White solid, m. p.: $178-180{ }^{\circ} \mathrm{C} ;{ }^{1} \mathrm{H}$ NMR (400 MHz, $\left.343 \mathrm{~K}, \mathrm{DMSO}-d_{6}\right): \delta=8.25(\mathrm{t}, J=1.9 \mathrm{~Hz}, 1 \mathrm{H}), 8.16-8.14(\mathrm{~m}, 1 \mathrm{H})$, 7.97-7.94 (m, 2H), 7.67-7.64 (m, 1H), 7.57-7.54 (m, 1H), $7.43(\mathrm{t}, J=7.9 \mathrm{~Hz}, 1 \mathrm{H}), 7.02(\mathrm{dd}, J=7.4,5.1 \mathrm{~Hz}, 1 \mathrm{H}), 6.63(\mathrm{dt}, J=$ 19.3, 10.3 Hz, 1H), 5.46 (brt, $J=10.1 \mathrm{~Hz}, 1 \mathrm{H}), 3.74(\mathrm{ddd}, J=12.2,10.6,6.6 \mathrm{~Hz}, 1 \mathrm{H}), 3.39$ (ddd, $J=12.2,10.7,5.8 \mathrm{~Hz}, 1 \mathrm{H})$, 2.89-2.81 (m, 1H), 2.61-2.53 (m, 1H), $1.36 \mathrm{ppm}(\mathrm{s}, 9 \mathrm{H}) ;{ }^{13} \mathrm{C}$ NMR (125 MHz, $\left.343 \mathrm{~K}, \mathrm{DMSO}-d_{6}\right): \delta=162.5(\mathrm{~d}, J=9.1 \mathrm{~Hz})$, 154.0 (br), 153.3, 147.1, 145.0, 141.2, 134.2, 134.1, 128.9, 125.9, 122.4, 122.1, 119.0, 118.4 (qt, J= 287.9, 36.6 Hz), 114.2 (m), 78.2, 52.7 (br), $47.7(\mathrm{t}, J=18.7 \mathrm{~Hz}), 45.4,27.8,22.8$ ppm; ${ }^{19} \mathrm{~F}$ NMR (376 MHz, $\left.343 \mathrm{~K}, \mathrm{DMSO}-d_{6}\right): \delta=-81.4(\mathrm{~s}, 3 \mathrm{~F}),-109.8$ (brd, $J=276.5 \mathrm{~Hz}, 1 \mathrm{~F}),-114.7$ ppm (dd, $J=274.4,19.1 \mathrm{~Hz}, 1 \mathrm{~F})$; IR (film): $\widetilde{v}=3344$ (br), 3069, 2977, 1704, 1662, 1595, 1532,

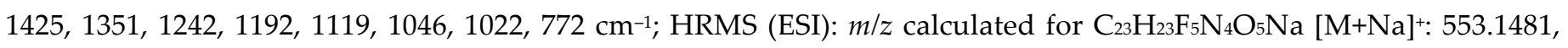
found: 553.1479; $[\alpha]_{D}^{25}=-53.3\left(c=0.25, \mathrm{CHCl}_{3}, 92 \%\right.$ ee sample); HPLC analysis (CHIRALPAK IC-3 $(\phi=0.46 \mathrm{~cm} \times 25$ $\mathrm{cm}$ ), 2-propanol $/ n$-hexane $=1 / 9$, flow rate $=1.0 \mathrm{~mL} / \mathrm{min}$, detection at $254 \mathrm{~nm}, \mathrm{t}_{\mathrm{R}}=29.9 \mathrm{~min}($ minor $), 33.6 \mathrm{~min}(\mathrm{major})$ ):
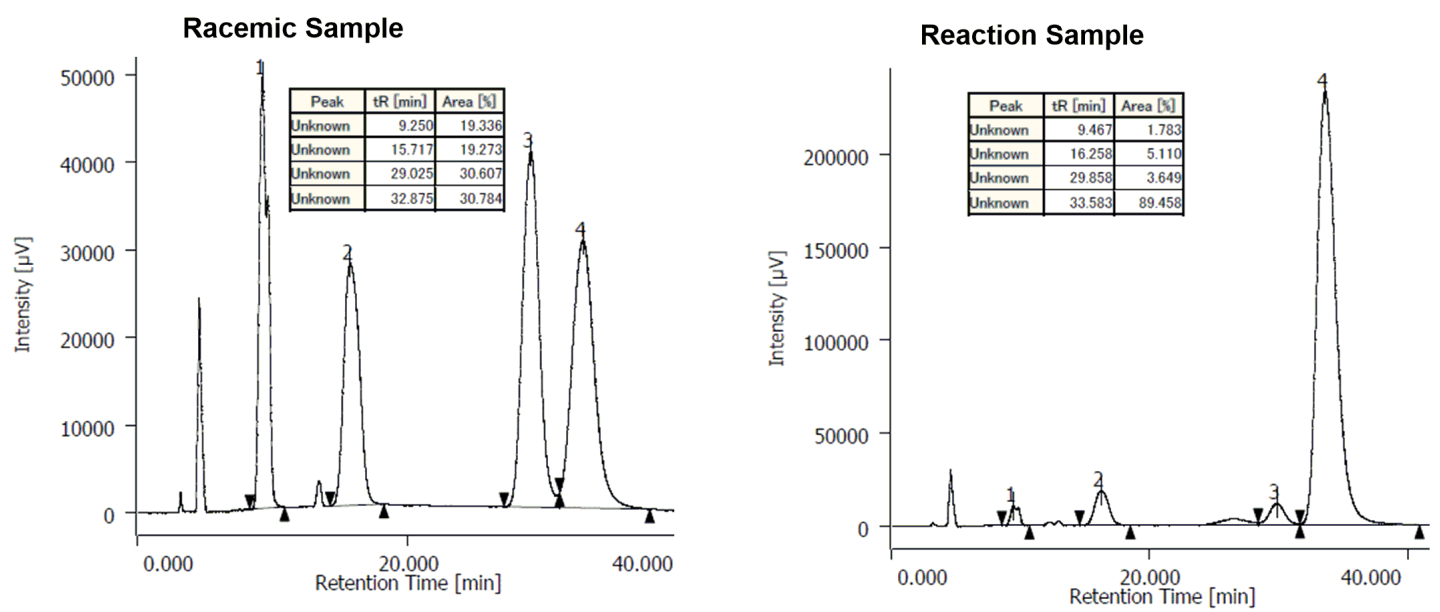

tert-Butyl ((1S,2S)-2-(2,3-dihydro-1H-pyrrolo[2,3-b]pyridine-1-carbonyl)-3,3,4,4,4-pentafluoro-1-(furan-2-yl)butyl)-

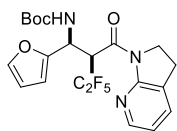
carbamate (120).

The reaction was performed according to procedure $\mathrm{C}$ at a reaction temperature of $-40{ }^{\circ} \mathrm{C}$, using $\left[\mathrm{Cu}\left(\mathrm{CH}_{3} \mathrm{CN}\right)_{4}\right] \mathrm{PF}_{6} \quad(3.7 \mathrm{mg}, \quad 0.01 \mathrm{mmol}, 10 \mathrm{~mol} \%), \quad(R)-(+)-5,5^{\prime}$-bis[di(3,5-xylyl)phosphino]-4,4'-bi-1,3-benzodioxole (DM-SEGPHOS) (8.7 mg, $0.012 \mathrm{mmol}, 12 \mathrm{~mol} \%$ ), anhydrous THF (0.10 mL, $0.5 \mathrm{M}), \mathrm{N}$-Boc imine 20 (39.4 mg), and Barton's base (0.1 M in THF, $0.1 \mathrm{~mL}, 0.01 \mathrm{mmol}, 10 \mathrm{~mol} \%)$. The diastereomeric ratio of the target material was initially identified as 13.5:1; after purification by flash column chromatography (hexane/ethyl acetate, $2: 1), 41.7 \mathrm{mg}(88 \%)$ of the desired pure diastereomer 120 were obtained.

White solid, m. p.: $96-98{ }^{\circ} \mathrm{C} ;{ }^{1} \mathrm{H}$ NMR $\left(400 \mathrm{MHz}, 343 \mathrm{~K}, \mathrm{DMSO}-\mathrm{d}_{6}\right): \delta=8.14-8.12(\mathrm{~m}, 1 \mathrm{H}), 7.65(\mathrm{dq}, J=7.3,1.3 \mathrm{~Hz}, 1 \mathrm{H})$, 7.44 (brs, 1H), 7.31 (dd, $J=1.7,0.8 \mathrm{~Hz}, 1 \mathrm{H}), 7.05(\mathrm{dd}, J=7.4,5.1 \mathrm{~Hz}, 1 \mathrm{H}), 6.54(\mathrm{dt}, J=18.5,10.9 \mathrm{~Hz}, 1 \mathrm{H}), 6.20(\mathrm{dd}, J=3.2$, $1.8 \mathrm{~Hz}, 1 \mathrm{H}), 6.17(\mathrm{~d}, J=3.2 \mathrm{~Hz}, 1 \mathrm{H}), 5.50$ (brt, $J=9.4 \mathrm{~Hz}, 1 \mathrm{H}), 3.88(\mathrm{ddd}, J=12.1,10.4,6.8 \mathrm{~Hz}, 1 \mathrm{H}), 3.72(\mathrm{ddd}, J=12.1$, 10.5, 6.2 Hz, 1H), 3.03-2.85 (m, 2H), 1.37 ppm (s, 9H); ${ }^{13} \mathrm{C}$ NMR (125 MHz, $\left.343 \mathrm{~K}, \mathrm{DMSO}-d 6\right): \delta=162.5(\mathrm{~d}, J=8.3 \mathrm{~Hz})$, 154.0 (br), 153.9, 152.3, 145.0, 141.4, 134.2, 126.2, 118.8, 118.4 (qt, J = 288.0, $36.7 \mathrm{~Hz}), 114.1$ (m), $109.9,106.6,78.1,46.9$ (t, $J$ = 18.7 Hz), 46.7 (br), 45.7, 27.8, 23.0 ppm; ${ }^{19} \mathrm{~F}$ NMR (376 MHz, $\left.343 \mathrm{~K}, \mathrm{DMSO}-d_{6}\right): \delta=-81.4$ (s, 3F), -110.8 (brd, J = 275.1 Hz, 1F), -114.4 ppm (dd, $J=275.1,18.0 \mathrm{~Hz}, 1 \mathrm{~F})$; IR (film): $\widetilde{v}=3344$ (br), 3059, 2979, 1712, 1666, 1595, 1501, 1478, 1426,

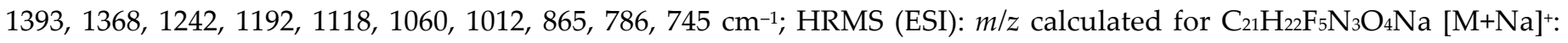
498.1423, found: 498.1422; $[\alpha]_{D}^{25}=-74.3\left(c=1.10, \mathrm{CHCl}_{3}, 99 \%\right.$ ee sample); HPLC analysis (CHIRALPAK IC $(\phi=0.46 \mathrm{~cm}$ x $25 \mathrm{~cm}$ ), 2-propanol $/ n$-hexane $=1 / 8$, flow rate $=1.0 \mathrm{~mL} / \mathrm{min}$, detection at $254 \mathrm{~nm}, \mathrm{tR}=72.0 \mathrm{~min}(\mathrm{major}), 102.4 \mathrm{~min}$ (minor)):
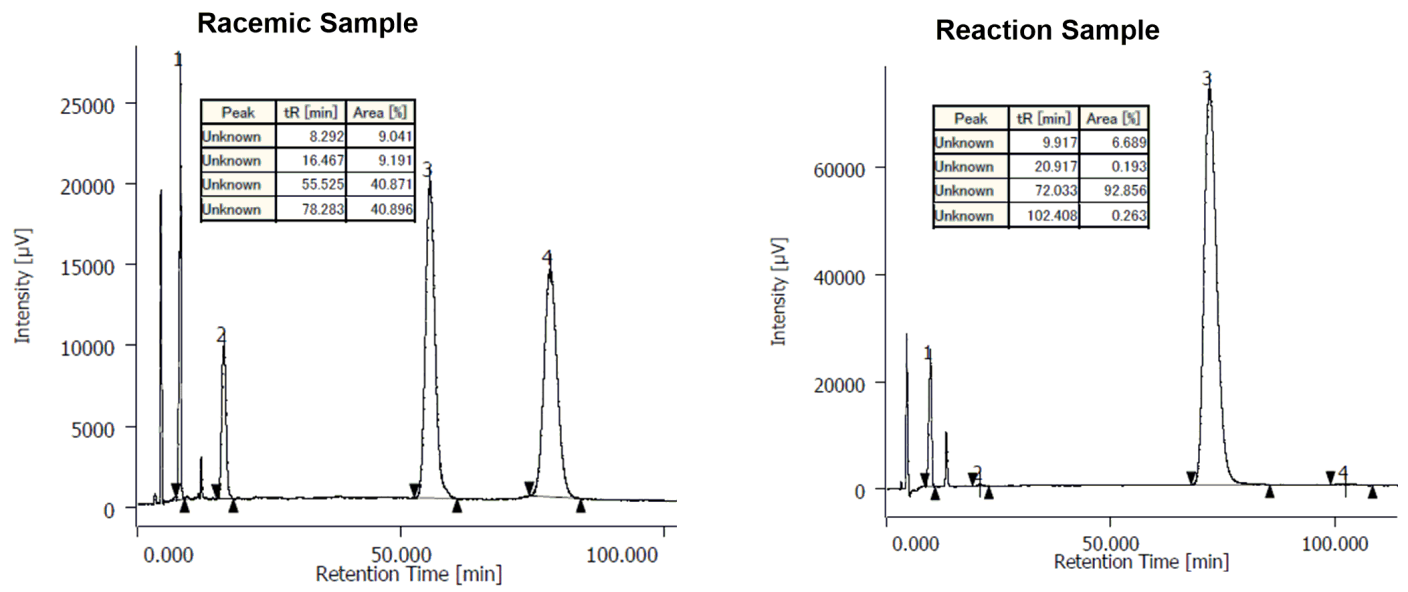

tert-Butyl ((3S,4R)-3-(2,3-dihydro-1H-pyrrolo[2,3-b]pyridine-1-carbonyl)-1,1,1,2,2-pentafluorononan-4-yl)carbamate (N) (12s).

The reaction was performed according to procedure $\mathrm{C}$ at a reaction temperature of $-20{ }^{\circ} \mathrm{C}$, using $\left[\mathrm{Cu}\left(\mathrm{CH}_{3} \mathrm{CN}\right)_{4}\right] \mathrm{PF}_{6} \quad(3.7 \mathrm{mg}, 0.01 \mathrm{mmol}, 10 \mathrm{~mol} \%), \quad(R)-(+)-5,5^{\prime}$-bis[di(3,5-xylyl)phosphino]-4,4'-bi-1,3-benzodioxole (DM-SEGPHOS) (8.7 mg, $0.012 \mathrm{mmol}, 12 \mathrm{~mol} \%)$, anhydrous THF (0.15 mL, $0.4 \mathrm{M})$, N-Boc imine $2 \mathrm{~s}$ (99 mg, 5.0 equiv.), and Barton's base $(0.1 \mathrm{M}$ in THF, $0.1 \mathrm{~mL}, 0.01 \mathrm{mmol}, 10 \mathrm{~mol} \%)$ to afford $39.9 \mathrm{mg}(83 \%)$ of the desired product $12 \mathrm{~s}$ as an 8:1 mixture of diastereomers after purification by flash column chromatography (hexane/ethyl acetate, 2:1). Analytical 
data for the major diastereomer are listed below:

Clear colorless oil; ${ }^{1} \mathrm{H}$ NMR $\left(400 \mathrm{MHz}, 343 \mathrm{~K}, \mathrm{DMSO}-\mathrm{d}_{6}\right): \delta=8.05$ (d, J=5.0 Hz, 1H), 7.68-7.65 (m, $\left.1 \mathrm{H}\right), 7.01(\mathrm{dd}, J=7.4$, $5.1 \mathrm{~Hz}, 1 \mathrm{H}), 6.69$ (brs, 1H), $6.19(\mathrm{ddd}, J=20.1,12.8,6.8 \mathrm{~Hz}, 1 \mathrm{H}), 4.18$ (brs, 1H), 4.10-3.94 (m, 2H), 3.07-3.03 (m, $2 \mathrm{H})$, 1.60-1.46 (m, 2H), $1.31(\mathrm{~s}, 9 \mathrm{H}), 1.26-1.11(\mathrm{~m}, 6 \mathrm{H}), 0.82 \mathrm{ppm}(\mathrm{t}, J=6.9 \mathrm{~Hz}, 3 \mathrm{H}) ;{ }^{13} \mathrm{C}$ NMR $\left(125 \mathrm{MHz}, 343 \mathrm{~K}, \mathrm{DMSO}-d_{6}\right): \delta=$ $163.2(\mathrm{~d}, J=8.4 \mathrm{~Hz}), 154.6$ (br), 154.3, 145.0, 134.1, 126.4, 118.5(3), 118.5 (qt, J = 287.5, $36.8 \mathrm{~Hz}), 114.4$ (m), $77.2,48.6$ (br), $47.0(\mathrm{t}, J=18.3 \mathrm{~Hz}), 45.8,30.8,30.2,27.9,24.7,23.0,21.5,13.3 \mathrm{ppm} ;{ }^{19} \mathrm{~F}$ NMR $\left(376 \mathrm{MHz}, 343 \mathrm{~K}, \mathrm{DMSO}-d_{6}\right): \delta=-82.0(\mathrm{~s}, 3 \mathrm{~F})$, -111.7 (brd, $J=272.7 \mathrm{~Hz}, 1 \mathrm{~F}),-114.3$ ppm (dd, $J=272.9,19.5 \mathrm{~Hz}, 1 \mathrm{~F})$; IR (film): $\widetilde{v}=3355$ (br), 3062, 2959, 2931, 2860, 1708,

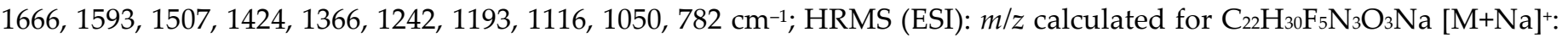
502.2100, found: 502.2096; $[\alpha]_{D}^{25}=-33.3\left(c=0.70, \mathrm{CHCl}_{3}, 99 \%\right.$ ee sample); HPLC analysis (CHIRALPAK IE $(\phi=0.46 \mathrm{~cm}$ x $25 \mathrm{~cm}$ ), 2-propanol $/ n$-hexane $=1 / 13$, flow rate $=1.0 \mathrm{~mL} / \mathrm{min}$, detection at $254 \mathrm{~nm}, \mathrm{t}=14.1 \mathrm{~min}(\mathrm{minor}), 16.9 \mathrm{~min}$ (major)):
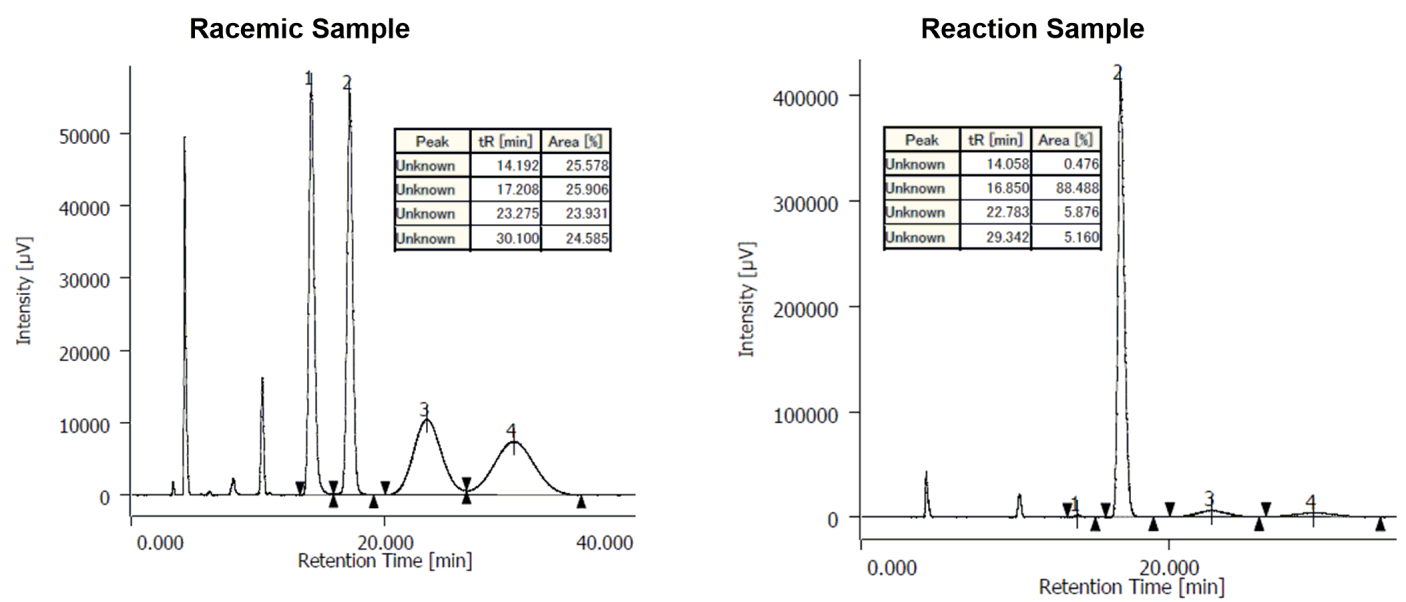

tert-Butyl ((1S,2S)-4-bromo-2-(2,3-dihydro-1H-pyrrolo[2,3-b]pyridine-1-carbonyl)-3,3,4,4-tetrafluoro-1-phenylbutyl)BrF carbamate (14a).

The reaction was performed according to procedure C using N-Boc imine 2a (123.2 mg, 6.0 equiv.), anhydrous THF (0.15 mL, $0.3 \quad \mathrm{M})$, and 4-Bromo-1-(2,3-dihydro-1H-pyrrolo[2,3-b]pyridin-1-yl)3,3,4,4-tetrafluorobutan-1-one (1d) (34.1 mg, 1.0 equiv.) instead of 1-(2,3-dihydro-1H-pyrrolo[2,3-b]pyridin-1-yl)3,3,4,4,4-pentafluorobutan-1-one (1c) to afford $53.0 \mathrm{mg}(97 \%)$ of the desired pure product 14a after purification by flash column chromatography (hexane/ethyl acetate, 2:1).

White solid, m. p.: $149-150{ }^{\circ} \mathrm{C} ;{ }^{1} \mathrm{H}$ NMR (400 MHz, $343 \mathrm{~K}$, DMSO-d6): $\delta=8.15-8.13$ (m, 1H), 7.55-7.52 (m, 2H), 7.26-7.23 (m, 2H), 7.10-7.06 (m, 1H), 7.03-6.99 (m, 3H), 6.54-6.44 (m, 1H), $5.33(\mathrm{t}, J=10.0 \mathrm{~Hz}, 1 \mathrm{H}), 3.69$ (ddd, J = 12.1, 10.6, 6.7 Hz, $1 \mathrm{H}), 3.32(\mathrm{ddd}, J=12.0,10.8,5.8 \mathrm{~Hz}, 1 \mathrm{H}), 2.85-2.77(\mathrm{~m}, 1 \mathrm{H}), 2.58-2.51(\mathrm{~m}, 1 \mathrm{H}), 1.36 \mathrm{ppm}(\mathrm{s}, 9 \mathrm{H}) ;{ }^{13} \mathrm{C} \mathrm{NMR}(125 \mathrm{MHz}$, 343 K, DMSO-d6): $\delta=163.0$ (d, J = 10.2 Hz), 154.0 (br), 153.6, 144.8, 139.2, 133.9, 127.6, 127.0, 126.9, 125.9, $118.6,117.6$ (tt, J $=312.5,42.3 \mathrm{~Hz}), 115.6(\mathrm{~m}), 77.8,53.6$ (br), $47.5(\mathrm{t}, J=19.0 \mathrm{~Hz}), 45.3,27.9,22.7 \mathrm{ppm} ;{ }^{19} \mathrm{~F} \mathrm{NMR}(376 \mathrm{MHz}, 343 \mathrm{~K}$, DMSO-d $): \delta=-61.7(\mathrm{~m}, 2 \mathrm{~F}),-101.4$ (brd, $J=266.6 \mathrm{~Hz}, 1 \mathrm{~F}),-108.3$ ppm $(\mathrm{dd}, J=266.5,19.5 \mathrm{~Hz}, 1 \mathrm{~F})$; IR (film): $\tilde{v}=3300$ (br), 3062, 3035, 3004, 2976, 2932, 1709, 1645, 1594, 1532, 1426, 1366, 1242, 1162, 1118, 1076, 761, 701 cm-1; HRMS (ESI): m/z calculated for $\mathrm{C}_{23} \mathrm{H}_{24} \mathrm{BrF}_{4} \mathrm{~N}_{3} \mathrm{O}_{3} \mathrm{Na}[\mathrm{M}+\mathrm{Na}]^{+}$: 568.0829, found: $568.0824 ;[\alpha]_{D}^{26}=-52.1\left(c=0.45, \mathrm{CHCl}_{3}, 97 \%\right.$ ee sample); 

HPLC analysis (CHIRALPAK IE $(\phi=0.46 \mathrm{~cm} \times 25 \mathrm{~cm}), 2$-propanol $/ n$-hexane $=1 / 9$, flow rate $=1.0 \mathrm{~mL} / \mathrm{min}$, detection at $254 \mathrm{~nm}, \mathrm{tR}=42.2 \mathrm{~min}$ (major), $48.0 \mathrm{~min}($ minor) $)$ :
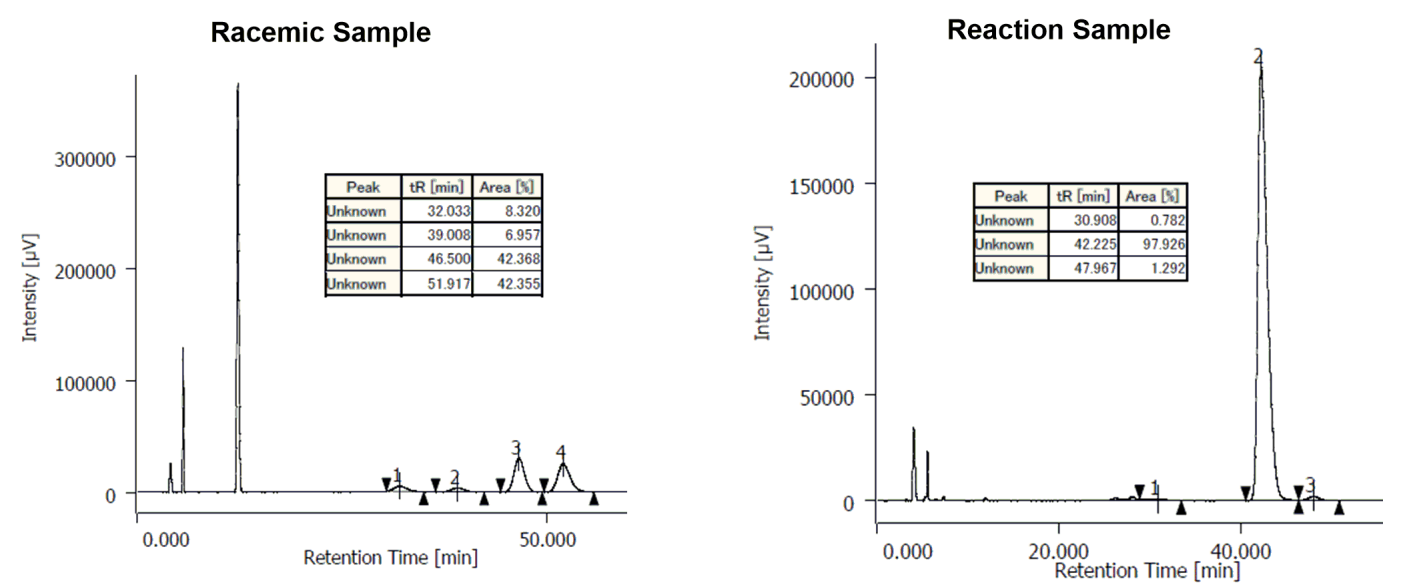

Benzyl ((1S,2S)-4-bromo-2-(2,3-dihydro-1H-pyrrolo[2,3-b]pyridine-1-carbonyl)-3,3,4,4-tetrafluoro-1-phenylbutyl)CbzHN $\mathrm{O}$ carbamate (14b).

The reaction was performed according to procedure $\mathrm{C}$ using $\mathrm{N}$-Cbz imine $2 \mathrm{~b}$ (143.6 mg, 6.0 equiv.) and 4-Bromo-1-(2,3-dihydro-1H-pyrrolo[2,3-b]pyridin-1-yl)-3,3,4,4-tetrafluorobutan-1-one (1d) (34.1 mg, 1.0 equiv.) instead of 1-(2,3-dihydro-1H-pyrrolo[2,3-b]pyridin-1-yl)-3,3,4,4,4-pentafluorobutan-1-one (1c) to afford $53.0 \mathrm{mg}$ (91\%) of the desired pure product $\mathbf{1 4 b}$ after purification by flash column chromatography (hexane/ethyl acetate, 2:1).

White solid, m. p.: $126-128{ }^{\circ} \mathrm{C}$; ${ }^{1} \mathrm{H}$ NMR (400 MHz, $343 \mathrm{~K}$, DMSO-d6): $\delta=8.14-8.11$ (m, 1H), 8.09 (brs, $\left.1 \mathrm{H}\right), 7.56-7.53$ (m, 1H), 7.35-7.25 (m, 7H), 7.11-6.99 (m, 4H), 6.60-6.50 (m, 1H), $5.40(\mathrm{t}, J=10.1 \mathrm{~Hz}, 1 \mathrm{H}), 5.05(\mathrm{~d}, J=12.7 \mathrm{~Hz}, 1 \mathrm{H}), 4.98(\mathrm{~d}, J=$ 12.7 Hz, 1H), 3.70 (ddd, $J=12.1,10.6,6.7 \mathrm{~Hz}, 1 \mathrm{H}), 3.34(\mathrm{ddd}, J=11.9,10.9,5.8 \mathrm{~Hz}, 1 \mathrm{H}), 2.86-2.78$ (m, 1H), $2.59-2.52 \mathrm{ppm}$ (m, 1H); ${ }^{13} \mathrm{C}$ NMR (125 MHz, $\left.343 \mathrm{~K}, \mathrm{DMSO}-d_{6}\right): \delta=162.8$ (d, J = 10.4 Hz), 154.7 (br), 153.6, 144.8, 138.8, 136.7, 133.9, 127.9, $127.5,127.3,127.1(3), 127.1,127.0(8), 125.9,118.6,117.5(\mathrm{tt}, J=312.4,42.1 \mathrm{~Hz}), 115.6(\mathrm{~m}), 65.2,54.1(\mathrm{br}), 47.4(\mathrm{t}, J=18.9$ $\mathrm{Hz}), 45.3,22.7 \mathrm{ppm} ;{ }^{19} \mathrm{~F}$ NMR (376 MHz, $\left.343 \mathrm{~K}, \mathrm{DMSO}-\mathrm{d}_{6}\right): \delta=-61.8(\mathrm{~m}, 2 \mathrm{~F}),-100.8(\mathrm{~d}, J=265.5 \mathrm{~Hz}, 1 \mathrm{~F}),-108.5 \mathrm{ppm}(\mathrm{dd}$, $J=266.2,18.0 \mathrm{~Hz}, 1 \mathrm{~F})$; IR (film): $\widetilde{v}=3324$ (br), 3062, 3032, 2956, 1716, 1652, 1594, 1540, 1423, 1241, 1204, 1148, 1115, 1077, $751 \mathrm{~cm}^{-1}$; HRMS (ESI): $m / z$ calculated for $\mathrm{C}_{26} \mathrm{H}_{22} \mathrm{BrF}_{4} \mathrm{~N}_{3} \mathrm{O}_{3} \mathrm{Na}[\mathrm{M}+\mathrm{Na}]^{+}: 602.0673$, found: $602.0662 ;[\alpha]_{D}^{26}=-52.5(c=0.30$, $\mathrm{CHCl}_{3}, 98 \%$ ee sample); HPLC analysis (CHIRALPAK IB-3 $(\phi=0.46 \mathrm{~cm} \times 25 \mathrm{~cm}$ ), 2-propanol $/ n$-hexane $=1 / 13$, flow rate $=1.0 \mathrm{~mL} / \mathrm{min}$, detection at $254 \mathrm{~nm}, \mathrm{t}_{\mathrm{R}}=20.8 \mathrm{~min}$ (minor), $25.5 \mathrm{~min}$ (major)):
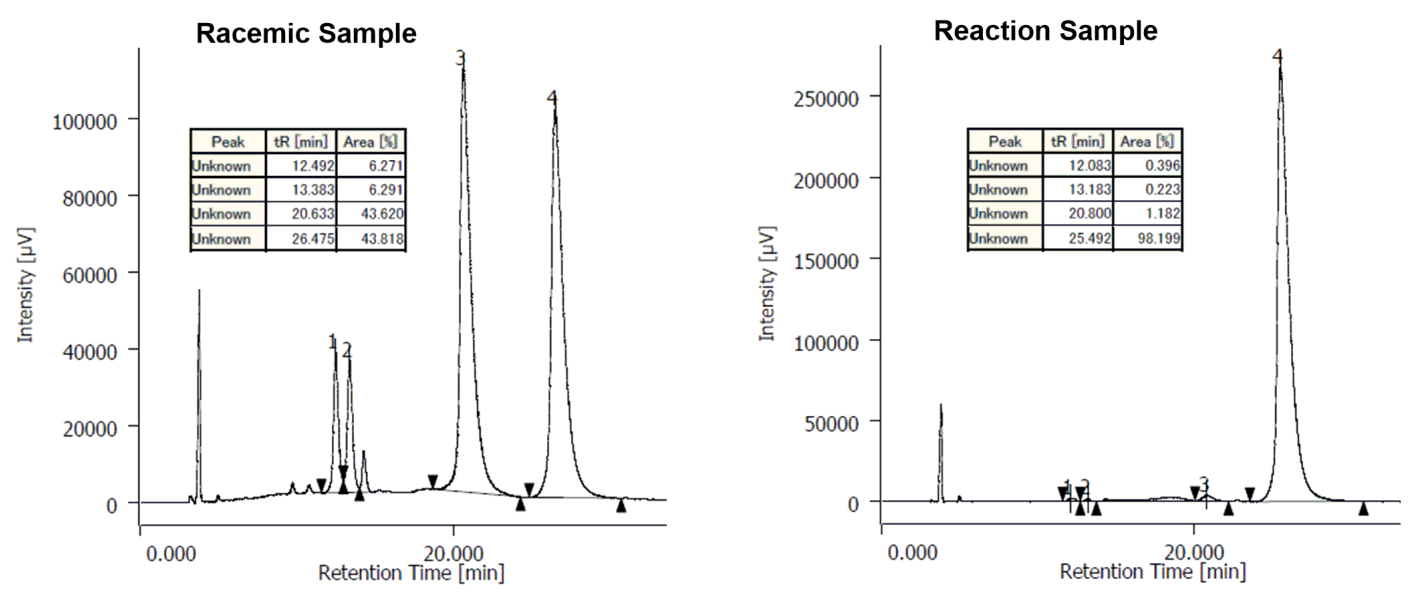


\section{1-(2,3-Dihydro-1H-pyrrolo[2,3-b]pyridin-1-yl)-4,4,4-trifluoro-3-(trifluoromethyl)but-2-en-1-one (13).}

A flame-dried $20 \mathrm{~mL}$ test tube equipped with a magnetic stirring bar and a 3-way glass stopcock was charged with $\quad\left[\mathrm{Cu}\left(\mathrm{CH}_{3} \mathrm{CN}\right)_{4}\right] \mathrm{PF}_{6} \quad(1.9 \quad \mathrm{mg}, \quad 0.005 \quad \mathrm{mmol}, \quad 10 \quad \mathrm{~mol} \%) \quad$ and (R)-(+)-2,2'-bis(di-para-tolylphosphino)-1,1'-binaphthyl (tol-BINAP) (4.1 mg, $0.006 \mathrm{mmol}, 12 \mathrm{~mol} \%)$ in a glove box under Ar atmosphere. Afterwards, the test tube was removed from the glove box and evacuated for 15 min. Then, it was backfilled with Ar and anhydrous THF $(0.15 \mathrm{~mL}, 0.3 \mathrm{M})$ was added at room temperature. The resulting clear colorless solution was stirred for $1 \mathrm{~h}$, before 1-(2,3-Dihydro-1H-pyrrolo[2,3- $b$ ]pyridin-1-yl)-3,4,4,4-tetrafluoro-3-(trifluoromethyl)butan-1-one (1f) (16.5 mg, $0.05 \mathrm{mmol}, 1.0$ equiv.), N-Cbz imine (2b) (23.9 mg, $0.1 \mathrm{mmol}, 2.0$ equiv.) and Barton's base (a $0.1 \mathrm{M}$ stock solution of Barton's base was prepared by diluting the commercial pure amine with the appropriate amount of freshly dried anhydrous THF and used for a maximum time period of 2 weeks before it was discarded; $0.1 \mathrm{M}$ in THF, $50 \mu \mathrm{L}, 0.005 \mathrm{mmol}, 10 \mathrm{~mol} \%$ ) were subsequently added. The reaction mixture was stirred for $22 \mathrm{~h}$ at ambient temperature before it was directly purified by flash column chromatography (hexane/ethyl acetate, 2:1) to yield amide 13 in quantitative yield.

White solid, m. p.: $86-88^{\circ} \mathrm{C} ;{ }^{1} \mathrm{H}$ NMR $\left(400 \mathrm{MHz}, 300 \mathrm{~K}, \mathrm{CDCl}_{3}\right): \delta=8.08(\mathrm{~d}, J=4.4 \mathrm{~Hz}, 1 \mathrm{H}), 7.61(\mathrm{~s}, 1 \mathrm{H}), 7.53(\mathrm{dq}, J=7.3$, $1.3 \mathrm{~Hz}, 1 \mathrm{H}), 6.97(\mathrm{dd}, J=7.4,5.1 \mathrm{~Hz}, 1 \mathrm{H}), 4.19(\mathrm{t}, J=8.4 \mathrm{~Hz}, 2 \mathrm{H}), 3.21 \mathrm{ppm}(\mathrm{t}, J=8.4 \mathrm{~Hz}, 2 \mathrm{H}) ;{ }^{13} \mathrm{C} \mathrm{NMR}(125 \mathrm{MHz}, 298 \mathrm{~K}$, $\left.\mathrm{CDCl}_{3}\right): \delta=160.0,154.7,146.6,137.8(\mathrm{~m}), 134.0,125.0,121.8$ (hept., $\left.J=33.5 \mathrm{~Hz}\right), 120.9(\mathrm{qq}, J=273.6,2.1 \mathrm{~Hz}), 120.3(\mathrm{q}, J=$ 275.0 Hz), 119.4, 44.8, 24.8 ppm; ${ }^{19} \mathrm{~F}$ NMR (376 MHz, $\left.300 \mathrm{~K}, \mathrm{CDCl}_{3}\right): \delta=-61.0$ (s, 3F), -64.9 ppm (s, 3F); IR (film): $\widetilde{v}=3045$, 2918, 1637, 1588, 1477, 1445, 1426, 1401, 1381, 1355, 1291, 1220, 1150, 986, $793 \mathrm{~cm}^{-1}$; HRMS (ESI): m/z calculated for $\mathrm{C}_{12} \mathrm{H}_{9} \mathrm{~F}_{6} \mathrm{~N}_{2} \mathrm{O}[\mathrm{M}+\mathrm{H}]^{+}:$311.0614, found: 311.0610 .

\section{4-7. Direct catalytic asymmetric Mannich-type reaction of $\alpha$-F amide 1e.}

\section{Procedure D:}

A flame-dried $20 \mathrm{~mL}$ test tube equipped with a magnetic stirring bar and a 3-way glass stopcock was charged with $\left[\mathrm{Cu}\left(\mathrm{CH}_{3} \mathrm{CN}\right)_{4}\right] \mathrm{PF}_{6}(3.7 \mathrm{mg}, 0.01 \mathrm{mmol}, 10 \mathrm{~mol} \%)$ and $\left(R_{P}\right)$-1-dicyclohexylphosphino-2-[(R)- $\alpha-(N, N-\operatorname{dimethylamino})-$ 2-(dicyclohexylphosphino)benzyl]ferrocene (Taniaphos-2) (8.5 mg, $0.012 \mathrm{mmol}, 12 \mathrm{~mol} \%$ ) in a glove box under Ar atmosphere. Afterwards, the test tube was removed from the glove box and evacuated for 15 min. Then, it was backfilled with Ar and freshly dried anhydrous THF $(0.20 \mathrm{~mL})$ was added at room temperature. The resulting clear orange solution was stirred for $1 \mathrm{~h}$.

Another flame-dried $20 \mathrm{~mL}$ test tube equipped with a magnetic stirring bar and a 3-way glass stopcock was charged with 1-(2,3-dihydro-1H-pyrrolo[2,3-b]pyridin-1-yl)-2-fluoroethan-1-one (1e) (18.0 mg, $0.1 \mathrm{mmol}, 1.0$ equiv.) and the corresponding freshly prepared $N-C b z$ imine $(\mathbf{2 b}, \mathbf{2 u - 2 z a})(0.2 \mathrm{mmol}, 2.0$ equiv.). The test tube was evacuated for 15 min, backfilled with Ar and freshly dried anhydrous THF $(0.20 \mathrm{~mL}, 0.2 \mathrm{M})$ was added at room temperature. Then, the separately prepared catalyst solution was added to the reaction mixture via a gas-tight syringe equipped with a 

stainless-steel needle. The resulting clear orange solution was cooled to $0{ }^{\circ} \mathrm{C}$ and finally Barton's base (a $0.1 \mathrm{M}$ solution of Barton's base was prepared immediately before use by diluting the commercial pure amine with the appropriate amount of freshly dried anhydrous THF; $0.1 \mathrm{M}$ in THF, $0.1 \mathrm{~mL}, 0.01 \mathrm{mmol}, 10 \mathrm{~mol} \%$ ) was added. The reaction mixture was stirred at $0{ }^{\circ} \mathrm{C}$ for $48 \mathrm{~h}$ before it was quenched by the addition of saturated aqueous $\mathrm{NH}_{4} \mathrm{Cl}$ solution and extracted three times with ethyl acetate. The combined extracts were dried over $\mathrm{Na}_{2} \mathrm{SO}_{4}$ and filtered. The diastereomeric ratio and the enantiomeric excess of the target material were determined by HPLC analysis of an aliquot, which was taken from the crude extracts and filtered through a short pad of silica. Then, the extracts were evaporated and analyzed by ${ }^{1} \mathrm{H}$ NMR to determine the conversion and to confirm the diastereomeric ratio (amide 1e could be recovered during the purification process; prolonged reaction times did not lead to enhanced conversions). Finally the crude reaction mixture was loaded directly onto a silica gel column and eluted with a suitable solution of $n$-hexane and ethyl acetate. Evaporation of solvent afforded the pure product.

Note that the NMR spectra of the Mannich products were recorded at elevated temperature (usually $50{ }^{\circ} \mathrm{C}$ ) in $\mathrm{MeCN}-\mathrm{d}_{3}$, as rotamers were observed in $\mathrm{CDCl}_{3}$ at room temperature. ${ }^{10}$

\section{Benzyl ((1S,2S)-3-(2,3-dihydro-1H-pyrrolo[2,3-b]pyridin-1-yl)2-fluoro-3-oxo-1-phenylpropyl)carbamate (15b).}

The reaction was performed according to procedure D using $N$-Cbz imine $2 \mathrm{~b}$ (47.8 $\mathrm{mg}$ ). After $48 \mathrm{~h}$ the conversion was determined to be $91 \%$ and the initial diastereomeric ratio of the product to be $8.9: 1$ (anti:syn). $32.9 \mathrm{mg}$ (79\%) of the desired pure diastereomer $\mathbf{1 5 b}$ were obtained after purification by flash column chromatography (hexane/ethyl acetate, 2:3).

White solid, m. p.: 147-149 ${ }^{\circ} \mathrm{C} ;{ }^{1} \mathrm{H}$ NMR (400 MHz, $\left.323 \mathrm{~K}, \mathrm{MeCN}-d_{3}\right): \delta=8.11-8.09$ (m, 1H), 7.59 (ddd, J = 7.4, $2.7,1.3 \mathrm{~Hz}$, 1H), 7.41-7.39 (m, 2H), 7.35-7.24 (m, 8H), $7.00(\mathrm{dd}, J=7.4,5.1 \mathrm{~Hz}, 1 \mathrm{H}), 6.79(\mathrm{dd}, J=48.4,6.5 \mathrm{~Hz}, 1 \mathrm{H}), 6.73$ (brs, $1 \mathrm{H}), 5.28$ $(\mathrm{ddd}, J=16.4,9.3,6.6 \mathrm{~Hz}, 1 \mathrm{H}), 5.04(\mathrm{~d}, J=12.6 \mathrm{~Hz}, 1 \mathrm{H}), 4.98(\mathrm{~d}, J=12.6 \mathrm{~Hz}, 1 \mathrm{H}), 4.08(\mathrm{ddd}, J=12.0,10.4,6.4 \mathrm{~Hz}, 1 \mathrm{H})$, $3.87(\mathrm{ddd}, J=12.0,10.4,6.5 \mathrm{~Hz}, 1 \mathrm{H}), 3.12-2.94 \mathrm{ppm}(\mathrm{m}, 2 \mathrm{H}) ;{ }^{13} \mathrm{C}$ NMR $\left(100 \mathrm{MHz}, 323 \mathrm{~K}, \mathrm{MeCN}-d_{3}\right): \delta=167.3(\mathrm{~d}, J=21.9$ Hz), 156.6 (br), 156.3, 146.8, 139.4, 138.3, 135.6, 129.6, 129.5, 129.1, 129.0, 128.8, 128.7, 128.2, $120.3,89.9$ (d, J = 180.3 Hz), 67.4, $58.0(\mathrm{~d}, J=24.3 \mathrm{~Hz}), 47.0,25.3 \mathrm{ppm} ;{ }^{19} \mathrm{~F} \mathrm{NMR}\left(376 \mathrm{MHz}, 323 \mathrm{~K}, \mathrm{MeCN}-d_{3}\right): \delta=-193.7 \mathrm{ppm}(\mathrm{d}, J=40.3 \mathrm{~Hz})$; IR (film): $\widetilde{v}=3330$ (br), 3062, 3032, 2956, 1719, 1671, 1594, 1508, 1474, 1427, 1308, 1240, 1043, 1026 cm-1 HRMS (ESI): m/z calculated for $\mathrm{C}_{24} \mathrm{H}_{23} \mathrm{FN}_{3} \mathrm{O}_{3}[\mathrm{M}+\mathrm{H}]^{+}: 420.1718$, found: $420.1713 ;[\alpha]_{D}^{25}=-56.5\left(c=1.00, \mathrm{CHCl}_{3}, 91 \%\right.$ ee sample); HPLC analysis (CHIRALPAK IA-3 $(\phi=0.46 \mathrm{~cm} \times 25 \mathrm{~cm}), 2$-propanol $/ n$-hexane $=1 / 4$, flow rate $=1.0 \mathrm{~mL} / \mathrm{min}$, detection at 254 $\mathrm{nm}, \mathrm{t} \mathrm{R}=23.2 \mathrm{~min}$ (major), $38.4 \mathrm{~min}($ minor) $)$ : 

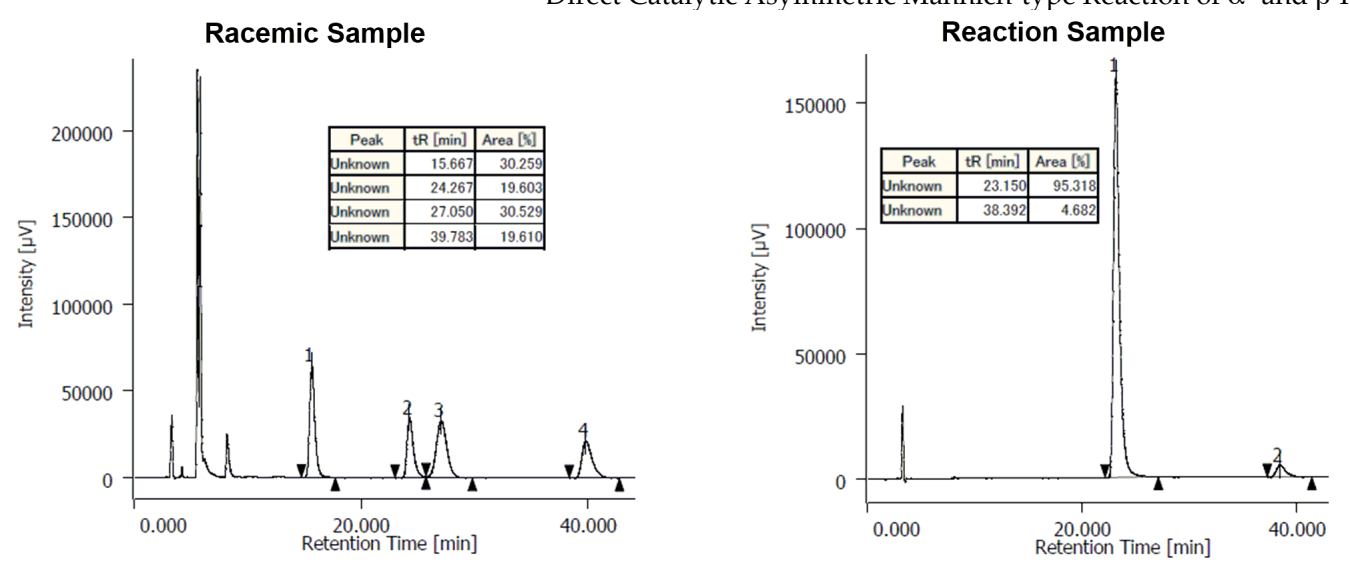

Benzyl ((1S,2S)-3-(2,3-dihydro-1H-pyrrolo[2,3-b]pyridin-1-yl)2-fluoro-3-oxo-1-(meta-tolyl)propyl)carbamate (15u).

CbzrN The reaction was performed according to procedure D using $\mathrm{N}$-Cbz imine $2 \mathbf{u}(50.6 \mathrm{mg})$. After $72 \mathrm{~h}$ the conversion was determined to be $90 \%$ and the initial diastereomeric ratio of the product to be 10.1:1 (anti:syn). $33.0 \mathrm{mg}(76 \%)$ of the desired pure diastereomer $\mathbf{1 5 u}$ were obtained after purification by flash column chromatography (hexane/ethyl acetate, 2:3).

White solid, m. p.: $123-125^{\circ} \mathrm{C}$; ${ }^{1} \mathrm{H}$ NMR (400 MHz, $\left.323 \mathrm{~K}, \mathrm{MeCN}-d_{3}\right): \delta=8.11-8.09(\mathrm{~m}, 1 \mathrm{H}), 7.58$ (ddd, J = 7.4, 2.8, 1.3 Hz, 1H), 7.36-7.16 (m, 8H), 7.09-7.07 (m, 1H), $7.00(\mathrm{dd}, J=7.4,5.1 \mathrm{~Hz}, 1 \mathrm{H}), 6.81(\mathrm{dd}, J=48.3,6.5 \mathrm{~Hz}, 1 \mathrm{H}), 6.67(\mathrm{brs}, 1 \mathrm{H}), 5.24$ $(\mathrm{ddd}, J=16.0,9.1,6.6 \mathrm{~Hz}, 1 \mathrm{H}), 5.04(\mathrm{~d}, J=12.6 \mathrm{~Hz}, 1 \mathrm{H}), 4.98(\mathrm{~d}, J=12.6 \mathrm{~Hz}, 1 \mathrm{H}), 4.07(\mathrm{ddd}, J=12.0,10.4,6.5 \mathrm{~Hz}, 1 \mathrm{H})$, $3.87(\mathrm{ddd}, J=12.0,10.4,6.4 \mathrm{~Hz}, 1 \mathrm{H}), 3.11-2.93(\mathrm{~m}, 2 \mathrm{H}), 2.30 \mathrm{ppm}(\mathrm{s}, 3 \mathrm{H}) ;{ }^{13} \mathrm{C}$ NMR $\left(100 \mathrm{MHz}, 323 \mathrm{~K}, \mathrm{MeCN}-d_{3}\right): \delta=167.4$ $(\mathrm{d}, J=22.0 \mathrm{~Hz}), 156.6$ (br), 156.3, 146.8, 139.3, 139.2, 138.4, 135.6, 129.8, 129.6, 129.4(4), 129.4, 129.1, 128.8, 128.2, 125.6, 120.3, $89.8(\mathrm{~d}, J=180.5 \mathrm{~Hz}), 67.4,58.0(\mathrm{~d}, J=25.1 \mathrm{~Hz}), 47.0,25.3,21.6 \mathrm{ppm} ;{ }^{19} \mathrm{~F}$ NMR $\left(376 \mathrm{MHz}, 323 \mathrm{~K}, \mathrm{MeCN}-d_{3}\right): \delta=$ -193.5 ppm (d, J = 42.7 Hz); IR (film): $\widetilde{v}=3333$ (br), 3059, 3025, 2952, 2915, 1724, 1672, 1594, 1507, 1475, 1428, 1307, 1281, 1239, 1045, 789, $753 \mathrm{~cm}^{-1}$; HRMS (ESI): $m / z$ calculated for $\mathrm{C}_{25} \mathrm{H}_{25} \mathrm{FN}_{3} \mathrm{O}_{3}[\mathrm{M}+\mathrm{H}]^{+}: 434.1874$, found: 434.1872; $[\alpha]_{D}^{26}=-68.8$ $\left(c=0.75, \mathrm{CHCl}_{3}, 93 \%\right.$ ee sample); HPLC analysis (CHIRALPAK IA $(\phi=0.46 \mathrm{~cm} \times 25 \mathrm{~cm}), 2$-propanol $/ n$-hexane $=1 / 9$, flow rate $=1.0 \mathrm{~mL} / \mathrm{min}$, detection at $254 \mathrm{~nm}, \mathrm{tr}=47.0 \mathrm{~min}$ (major), $73.7 \mathrm{~min}(\mathrm{minor})$ ):
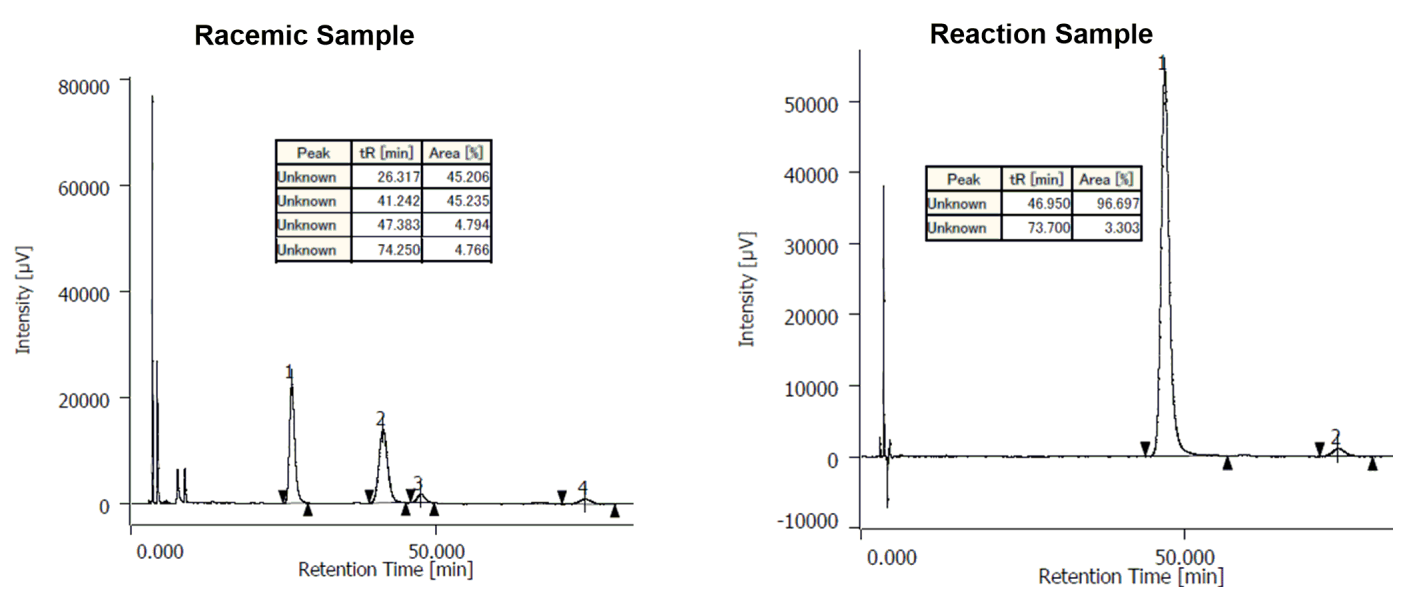

Benzyl ((1S,2S)-3-(2,3-dihydro-1H-pyrrolo[2,3-b]pyridin-1-yl)2-fluoro-3-oxo-1-(para-tolyl)propyl)carbamate (15v).

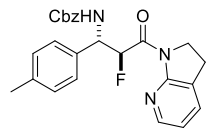

The reaction was performed according to procedure D using $\mathrm{N}-\mathrm{Cbz}$ imine $2 \mathbf{v}$ (50.6 mg). After $48 \mathrm{~h}$ the conversion was determined to be $89 \%$ and the initial diastereomeric ratio of the product to be $9.4: 1$ (anti:syn). $30.0 \mathrm{mg}(70 \%)$ of the desired pure diastereomer $15 \mathbf{v}$ were obtained after purification by flash column 
chromatography (hexane/ethyl acetate, 2:3).

White solid, m. p.: $129-130{ }^{\circ} \mathrm{C}$; ${ }^{1} \mathrm{H}$ NMR $\left(400 \mathrm{MHz}, 323 \mathrm{~K}, \mathrm{MeCN}-d_{3}\right): \delta=8.10-8.08(\mathrm{~m}, 1 \mathrm{H}), 7.58(\mathrm{ddd}, J=7.4,2.7,1.3 \mathrm{~Hz}$, $1 \mathrm{H}), 7.35-7.30(\mathrm{~m}, 3 \mathrm{H}), 7.27-7.23(\mathrm{~m}, 4 \mathrm{H}), 7.13(\mathrm{~d}, J=7.9 \mathrm{~Hz}, 2 \mathrm{H}), 7.00(\mathrm{dd}, J=7.4,5.1 \mathrm{~Hz}, 1 \mathrm{H}), 6.77(\mathrm{dd}, J=48.4,6.5 \mathrm{~Hz}$, 1H), 6.66 (brs, 1H), $5.23(\mathrm{ddd}, J=16.4,9.1,6.6 \mathrm{~Hz}, 1 \mathrm{H}), 5.04(\mathrm{~d}, J=12.6 \mathrm{~Hz}, 1 \mathrm{H}), 4.98(\mathrm{~d}, J=12.6 \mathrm{~Hz}, 1 \mathrm{H}), 4.07(\mathrm{ddd}, J=$ 12.0, 10.3, $6.5 \mathrm{~Hz}, 1 \mathrm{H}), 3.87(\mathrm{ddd}, J=12.0,10.3,6.5 \mathrm{~Hz}, 1 \mathrm{H}), 3.11-2.93(\mathrm{~m}, 2 \mathrm{H}), 2.29 \mathrm{ppm}(\mathrm{s}, 3 \mathrm{H}){ }^{13} \mathrm{C} \mathrm{NMR}(100 \mathrm{MHz}$, $\left.323 \mathrm{~K}, \mathrm{MeCN}-d_{3}\right): \delta=167.4(\mathrm{~d}, J=21.9 \mathrm{~Hz}), 156.6(\mathrm{br}), 156.3,146.8,139.0,138.3,136.3,135.6,130.1,129.6,129.0,128.8$, 128.6, 128.2, 120.2, $89.9(\mathrm{~d}, J=180.4 \mathrm{~Hz}), 67.4,57.7(\mathrm{~d}, J=25.5 \mathrm{~Hz}), 47.0,25.3,21.2 \mathrm{ppm} ;{ }^{19} \mathrm{~F} \mathrm{NMR}(376 \mathrm{MHz}, 323 \mathrm{~K}$, MeCN-d3): $\delta=-193.7$ ppm (d, J = 41.0 Hz); IR (film): $\widetilde{v}=3330$ (br), 3062, 3028, 3011, 2956, 2922, 1723, 1672, 1594, 1509, 1475, 1428, 1307, 1282, 1240, 1043, 1026, 774, $751 \mathrm{~cm}^{-1}$; HRMS (ESI): m/z calculated for $\mathrm{C}_{25} \mathrm{H}_{25} \mathrm{FN}_{3} \mathrm{O}_{3}\left[\mathrm{M}+\mathrm{H}^{+}: 434.1874\right.$, found: $434.1869 ;[\alpha]_{D}^{26}=-66.3\left(c=1.05, \mathrm{CHCl}_{3}, 93 \%\right.$ ee sample); HPLC analysis (CHIRALPAK IA $(\phi=0.46 \mathrm{~cm} \times 25 \mathrm{~cm})$, 2-propanol $/ n$-hexane $=1 / 4$, flow rate $=1.0 \mathrm{~mL} / \mathrm{min}$, detection at $254 \mathrm{~nm}, \mathrm{t}_{\mathrm{R}}=23.7 \mathrm{~min}$ (major), $43.2 \mathrm{~min}(\mathrm{minor})$ ):
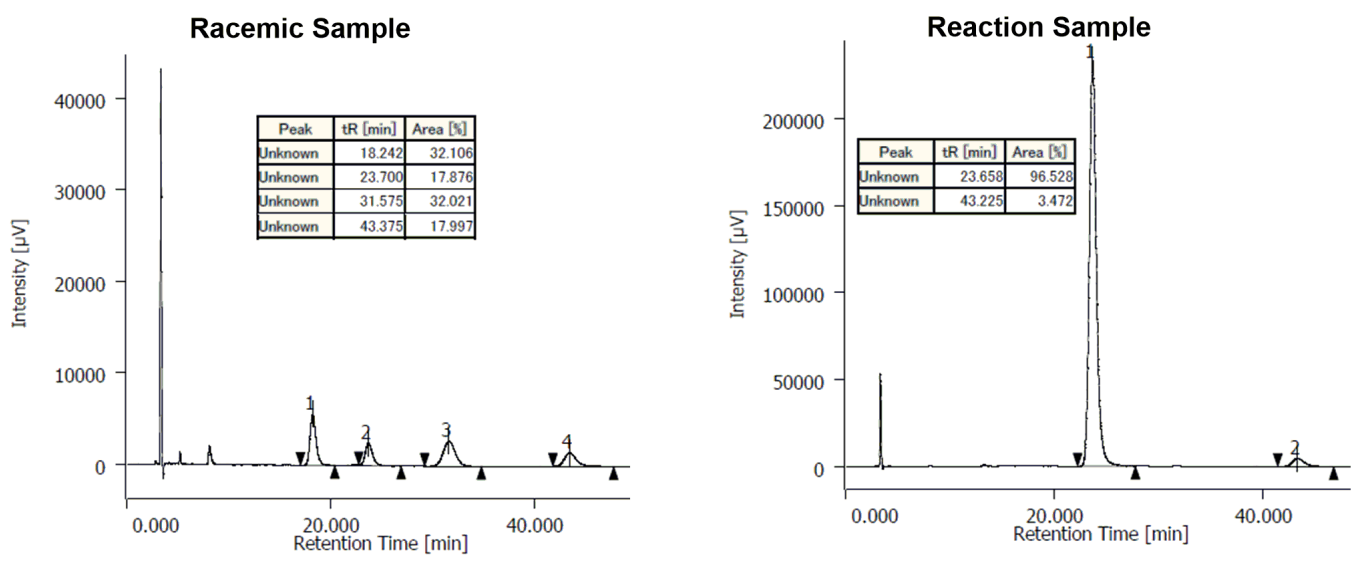

Benzyl ((1S,2S)-3-(2,3-dihydro-1H-pyrrolo[2,3-b]pyridin-1-yl)2-fluoro-1-(3-methoxyphenyl)-3-oxopropyl)carbamate

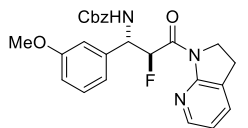
(15w).

The reaction was performed according to procedure D using $\mathrm{N}-\mathrm{Cbz}$ imine $2 \mathbf{w}$ (53.8 mg). After $48 \mathrm{~h}$ the conversion was determined to be $75 \%$ and the initial diastereomeric ratio of the product to be $8.9: 1$ (anti:syn). 25.0 $\mathrm{mg}(56 \%)$ of the desired pure diastereomer $15 \mathrm{w}$ were obtained after purification by flash column chromatography (hexane/ethyl acetate, 2:3).

White solid, m. p.: $143-145{ }^{\circ} \mathrm{C} ;{ }^{1} \mathrm{H}$ NMR $\left(400 \mathrm{MHz}, 323 \mathrm{~K}, \mathrm{MeCN}-d_{3}\right): \delta=8.09(\mathrm{dd}, J=5.1,0.7 \mathrm{~Hz}, 1 \mathrm{H}), 7.60-7.57(\mathrm{~m}, 1 \mathrm{H})$, 7.36-7.21 (m, 6H), $7.00(\mathrm{dd}, J=7.4,5.1 \mathrm{~Hz}, 1 \mathrm{H}), 6.97-6.95(\mathrm{~m}, 2 \mathrm{H}), 6.83-6.80(\mathrm{~m}, 1 \mathrm{H}), 6.81(\mathrm{dd}, J=48.3,6.4 \mathrm{~Hz}, 1 \mathrm{H}), 6.68$ (brs, 1H), $5.26(\mathrm{ddd}, J=16.0,9.3,6.5 \mathrm{~Hz}, 1 \mathrm{H}), 5.05(\mathrm{~d}, J=12.6 \mathrm{~Hz}, 1 \mathrm{H}), 4.99$ (d, J = 12.6 Hz, 1H), $4.08(\mathrm{ddd}, J=12.2,10.2$, $6.5 \mathrm{~Hz}, 1 \mathrm{H}), 3.87(\mathrm{ddd}, J=12.0,10.5,6.4 \mathrm{~Hz}, 1 \mathrm{H}), 3.77$ (s, 3H), 3.12-2.93 ppm (m, 2H); ${ }^{13} \mathrm{C} \mathrm{NMR}(125 \mathrm{MHz}, 323 \mathrm{~K}$, MeCN-d3): $\delta=167.2(\mathrm{~d}, J=21.9 \mathrm{~Hz}), 161.0,156.6$ (br), 156.3, 146.8, 140.9, 138.3, 135.6, 130.6, 129.5, 129.0, 128.8, 128.2, 120.8, 120.2, 114.7, 114.4, 89.8 (d, $J=180.6 \mathrm{~Hz}), 67.4,57.9$ (brd, $J=24.0 \mathrm{~Hz}), 56.1,47.0,25.2$ ppm; ${ }^{19} \mathrm{~F} \mathrm{NMR}(376 \mathrm{MHz}, 323$ K, MeCN-d3): $\delta=-193.6$ ppm (d, J = 42.1 Hz); IR (film): $\widetilde{v}=3333$ (br), 3062, 3014, 2959, 1724, 1673, 1595, 1495, 1428, 1308, 1264, 1240, 1167, 1040, $754 \mathrm{~cm}^{-1}$; HRMS (ESI): $m / z$ calculated for $\mathrm{C}_{25} \mathrm{H}_{25} \mathrm{FN}_{3} \mathrm{O}_{4}[\mathrm{M}+\mathrm{H}]^{+}: 450.1824$, found: 450.1820 ; $[\alpha]_{D}^{26}=$ -64.7 $\left(c=0.90, \mathrm{CHCl}_{3}, 91 \%\right.$ ee sample); HPLC analysis (CHIRALPAK IA $(\phi=0.46 \mathrm{~cm} \times 25 \mathrm{~cm}), 2$-propanol $/ n$-hexane $=$ $1 / 6$, flow rate $=1.0 \mathrm{~mL} / \mathrm{min}$, detection at $254 \mathrm{~nm}, \mathrm{t}_{\mathrm{R}}=44.9 \mathrm{~min}$ (major), $63.8 \mathrm{~min}$ (minor)): 

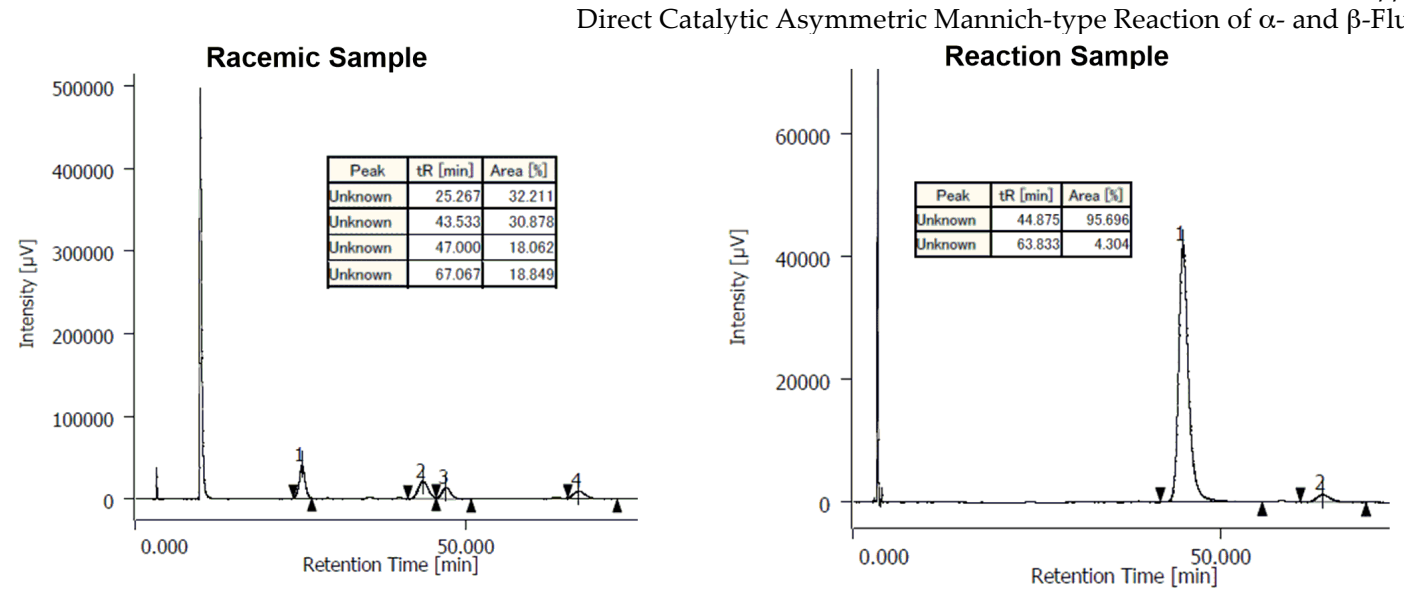

Benzyl ((1S,2S)-3-(2,3-dihydro-1H-pyrrolo[2,3-b]pyridin-1-yl)2-fluoro-1-(4-methoxyphenyl)-3-oxopropyl)carbamate

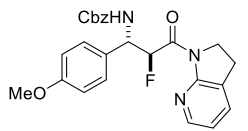
(15x).

The reaction was performed according to procedure D using $N$-Cbz imine $2 \mathbf{x}(53.8 \mathrm{mg})$. After $48 \mathrm{~h}$ the conversion was determined to be $80 \%$ and the initial diastereomeric ratio of the product to be $9.5: 1$ (anti:syn). $29.0 \mathrm{mg}$ $(65 \%)$ of the desired pure diastereomer $15 x$ were obtained after purification by flash column chromatography (hexane/ethyl acetate, 2:3).

White solid, m. p.: $158-160{ }^{\circ} \mathrm{C}$; ${ }^{1} \mathrm{H}$ NMR $\left(400 \mathrm{MHz}, 323 \mathrm{~K}, \mathrm{MeCN}-d_{3}\right): \delta=8.10-8.08(\mathrm{~m}, 1 \mathrm{H}), 7.58(\mathrm{ddd}, J=7.4,2.7,1.3 \mathrm{~Hz}$, 1H), 7.36-7.24 (m, 7H), $7.00(\mathrm{dd}, J=7.4,5.1 \mathrm{~Hz}, 1 \mathrm{H}), 6.88-6.85(\mathrm{~m}, 2 \mathrm{H}), 6.77(\mathrm{dd}, J=48.5,6.5 \mathrm{~Hz}, 1 \mathrm{H}), 6.63(\mathrm{brs}, 1 \mathrm{H}), 5.22$ $(\mathrm{ddd}, J=16.1,9.2,6.4 \mathrm{~Hz}, 1 \mathrm{H}), 5.04(\mathrm{~d}, J=12.6 \mathrm{~Hz}, 1 \mathrm{H}), 4.98(\mathrm{~d}, J=12.6 \mathrm{~Hz}, 1 \mathrm{H}), 4.07(\mathrm{ddd}, J=12.0,10.4,6.5 \mathrm{~Hz}, 1 \mathrm{H})$, $3.87(\mathrm{ddd}, J=12.2,10.2,6.4 \mathrm{~Hz}, 1 \mathrm{H}), 3.76(\mathrm{~s}, 3 \mathrm{H}), 3.11-2.94 \mathrm{ppm}(\mathrm{m}, 2 \mathrm{H}) ;{ }^{13} \mathrm{C}$ NMR $\left(100 \mathrm{MHz}, 323 \mathrm{~K}, \mathrm{MeCN}-d_{3}\right): \delta=167.4$ $(\mathrm{d}, J=21.8 \mathrm{~Hz}), 160.7,156.6$ (br), 156.3, 146.8, 138.4, 135.6, 131.3, 129.9, 129.6, 129.0, 128.8, 128.2, 120.2, 115.0, 89.9 (d, J = $180.4 \mathrm{~Hz}), 67.4,57.4(\mathrm{~d}, J=25.1 \mathrm{~Hz}), 56.1,47.0,25.3 \mathrm{ppm} ;{ }^{19} \mathrm{~F}$ NMR $\left(376 \mathrm{MHz}, 323 \mathrm{~K}, \mathrm{MeCN}-d_{3}\right): \delta=-193.5 \mathrm{ppm}(\mathrm{d}, J=$ 39.8 Hz); IR (film): $\widetilde{v}=3333$ (br), 3062, 3028, 3011, 2956, 2932, 1718, 1671, 1594, 1513, 1428, 1307, 1241, 1179, 1030, 771, 753 $\mathrm{cm}^{-1}$; HRMS (ESI): $\mathrm{m} / z$ calculated for $\mathrm{C}_{25} \mathrm{H}_{25} \mathrm{FN}_{3} \mathrm{O}_{4}[\mathrm{M}+\mathrm{H}]^{+}: 450.1824$, found: 450.1822; $[\alpha]_{D}^{26}=-70.6\left(c=0.55, \mathrm{CHCl}_{3}\right.$, 90\% ee sample); HPLC analysis (CHIRALPAK IA $(\phi=0.46 \mathrm{~cm} \times 25 \mathrm{~cm})$, 2-propanol $/ n$-hexane $=1 / 4$, flow rate $=1.0$ $\mathrm{mL} / \mathrm{min}$, detection at $254 \mathrm{~nm}, \mathrm{tr}=31.7 \mathrm{~min}$ (major), $63.4 \mathrm{~min}$ (minor)):
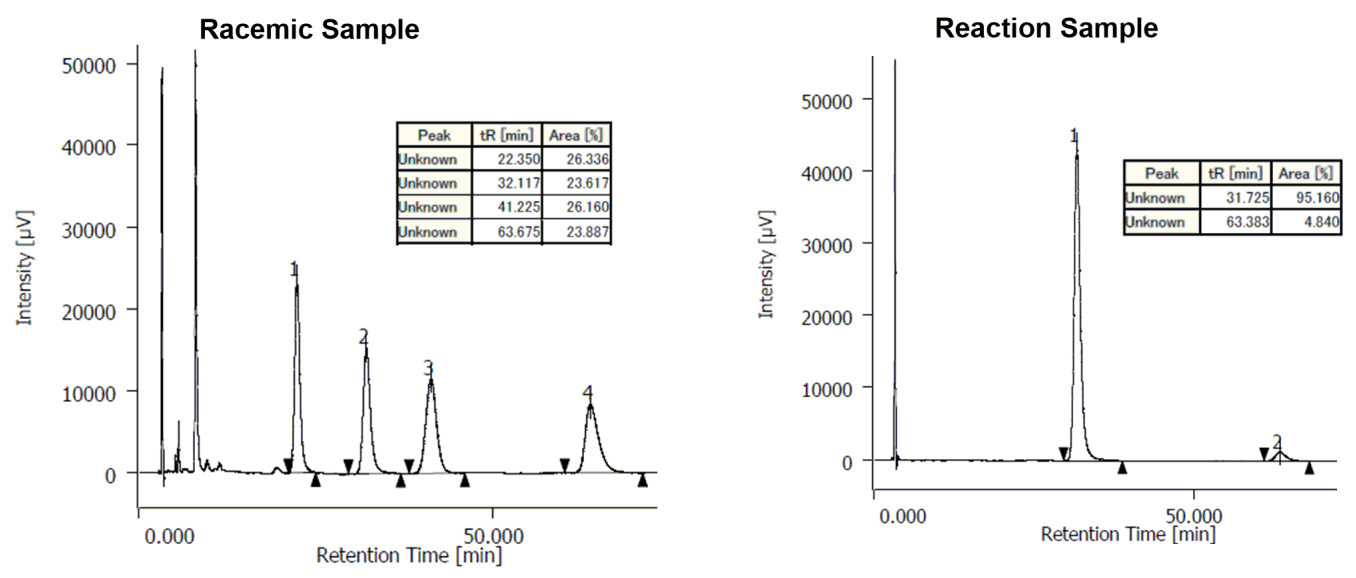

Benzyl ((1S,2S)-3-(2,3-dihydro-1H-pyrrolo[2,3-b]pyridin-1-yl)2-fluoro-1-(4-chlorophenyl)-3-oxopropyl)carbamate

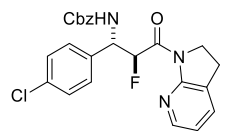

(15y).

The reaction was performed according to procedure D using $\mathrm{N}-\mathrm{Cbz}$ imine $2 \mathrm{y}(54.7 \mathrm{mg})$. After $48 \mathrm{~h}$ the conversion was determined to be $70 \%$ and the initial diastereomeric ratio of the product to be 6.0:1 (anti:syn). $25.0 \mathrm{mg}$ $(55 \%)$ of the desired pure diastereomer $15 y$ were obtained after purification by careful flash column chromatography (hexane/ethyl acetate, 3:2).

White solid, m. p.: $177-179{ }^{\circ} \mathrm{C}$; ${ }^{1} \mathrm{H}$ NMR $\left(400 \mathrm{MHz}, 323 \mathrm{~K}, \mathrm{MeCN}-d_{3}\right): \delta=8.09-8.07$ (m, $\left.1 \mathrm{H}\right), 7.60$ (ddd, J = 7.4, 2.7, $1.3 \mathrm{~Hz}$, 1H), 7.38-7.25 (m, 9H), $7.00(\mathrm{dd}, J=7.4,5.1 \mathrm{~Hz}, 1 \mathrm{H}), 6.77(\mathrm{dd}, J=48.4,6.1 \mathrm{~Hz}, 1 \mathrm{H}), 6.71$ (brs, $1 \mathrm{H}), 5.29$ (ddd, $J=17.2,8.9$, $6.1 \mathrm{~Hz}, 1 \mathrm{H}), 5.05(\mathrm{~d}, J=12.6 \mathrm{~Hz}, 1 \mathrm{H}), 4.99(\mathrm{~d}, J=12.6 \mathrm{~Hz}, 1 \mathrm{H}), 4.07(\mathrm{ddd}, J=12.0,10.3,6.5 \mathrm{~Hz}, 1 \mathrm{H}), 3.88(\mathrm{ddd}, J=12.0$, 10.4, $6.5 \mathrm{~Hz}, 1 \mathrm{H}), 3.13-2.95$ ppm (m, 2H); ${ }^{13} \mathrm{C}$ NMR (125 MHz, $\left.323 \mathrm{~K}, \mathrm{MeCN}-d_{3}\right): \delta=166.9$ (d, J = $\left.21.8 \mathrm{~Hz}\right), 156.6$ (br), $156.2,146.8,138.2,138.1,135.6,134.5,130.5,129.6,129.5,129.1,128.8,128.2,120.3,89.8(\mathrm{~d}, J=181.0 \mathrm{~Hz}), 67.5,57.3(\mathrm{~d}, J=$ $24.8 \mathrm{~Hz}), 46.9,25.3 \mathrm{ppm} ;{ }^{19} \mathrm{~F}$ NMR $\left(376 \mathrm{MHz}, 323 \mathrm{~K}, \mathrm{MeCN}-d_{3}\right): \delta=-194.4 \mathrm{ppm}(\mathrm{d}, J=36.3 \mathrm{~Hz})$; IR (film): $\widetilde{v}=3329$ (br), 3059, 3028, 2959, 2918, 1721, 1671, 1594, 1510, 1493, 1427, 1308, 1240, 1043, 1014, $772 \mathrm{~cm}^{-1}$; HRMS (ESI): m/z calculated for $\mathrm{C}_{24} \mathrm{H}_{22} \mathrm{ClFN}_{3} \mathrm{O}_{3}[\mathrm{M}+\mathrm{H}]^{+}: 454.1328$ found: $454.1326 ;[\alpha]_{D}^{25}=-86.6\left(c=0.40, \mathrm{CHCl}_{3}, 92 \%\right.$ ee sample); HPLC analysis (CHIRALPAK IA $(\phi=0.46 \mathrm{~cm} \times 25 \mathrm{~cm}), 2$-propanol $/ n$-hexane $=1 / 4$, flow rate $=1.0 \mathrm{~mL} / \mathrm{min}$, detection at $254 \mathrm{~nm}$, $\mathrm{tR}=$ $24.9 \min$ (major), $66.6 \mathrm{~min}$ (minor)):
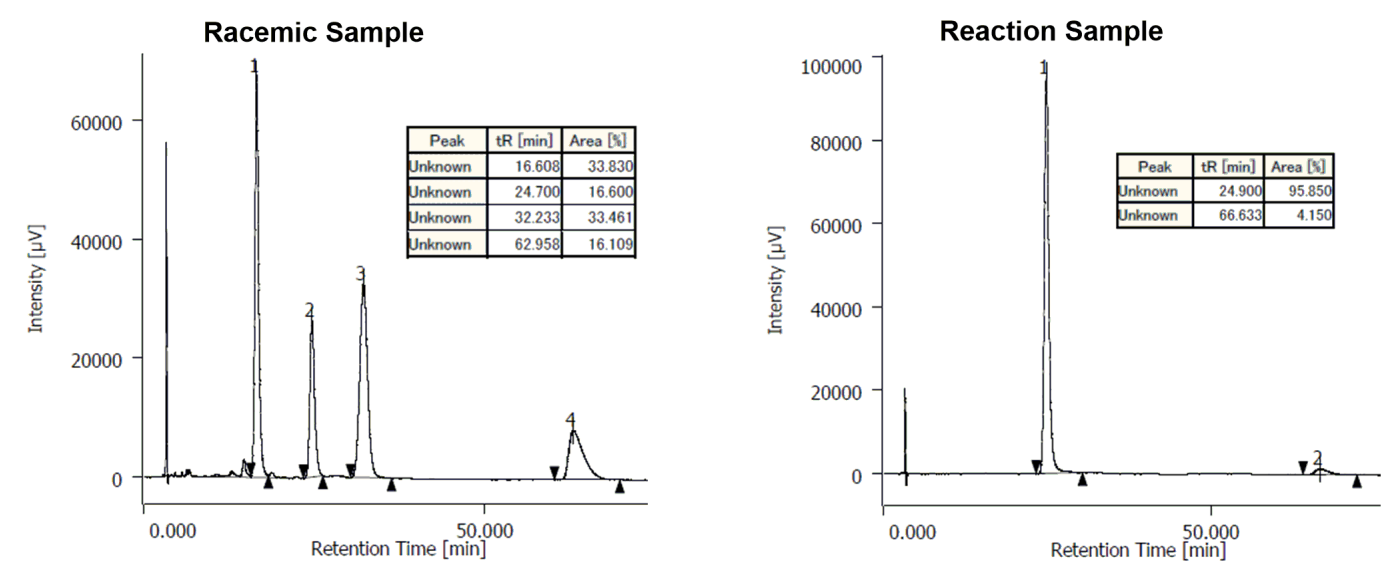

Benzyl ((1S,2S)-3-(2,3-dihydro-1H-pyrrolo[2,3-b]pyridin-1-yl)2-fluoro-1-(4-(methylthio)phenyl)-3-oxopropyl)-

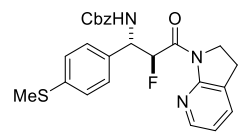
carbamate (15z).

The reaction was performed according to procedure D using $\mathrm{N}-\mathrm{Cbz}$ imine $2 \mathrm{z}(57.1 \mathrm{mg})$. After $48 \mathrm{~h}$ the conversion was determined to be $70 \%$ and the initial diastereomeric ratio of the product to be 5.7:1 (anti:syn). $23.6 \mathrm{mg}$ (51\%) of the desired pure diastereomer $\mathbf{1 5 z}$ were obtained after purification by flash column chromatography (hexane/ethyl acetate, 3:5).

White solid, m. p.: $174-177^{\circ} \mathrm{C}$; ${ }^{1} \mathrm{H}$ NMR $\left(400 \mathrm{MHz}, 323 \mathrm{~K}, \mathrm{MeCN}-d_{3}\right): \delta=8.09-8.07$ (m, 1H), 7.58 (ddd, J = 7.4, 2.7, 1.3 Hz, 1H), 7.36-7.19 (m, 9H), $7.00(\mathrm{dd}, J=7.4,5.1 \mathrm{~Hz}, 1 \mathrm{H}), 6.78(\mathrm{dd}, J=48.4,6.3 \mathrm{~Hz}, 1 \mathrm{H}), 6.68(\mathrm{brs}, 1 \mathrm{H}), 5.25$ (ddd, J = 16.4, 9.0, $6.2 \mathrm{~Hz}, 1 \mathrm{H}), 5.05(\mathrm{~d}, J=12.6 \mathrm{~Hz}, 1 \mathrm{H}), 4.99(\mathrm{~d}, J=12.6 \mathrm{~Hz}, 1 \mathrm{H}), 4.07(\mathrm{ddd}, J=12.0,10.4,6.6 \mathrm{~Hz}, 1 \mathrm{H}), 3.88(\mathrm{ddd}, J=12.0$, 10.4, $6.4 \mathrm{~Hz}, 1 \mathrm{H}), 3.12-2.94(\mathrm{~m}, 2 \mathrm{H}), 2.45 \mathrm{ppm}(\mathrm{s}, 3 \mathrm{H}) ;{ }^{13} \mathrm{C} \mathrm{NMR}\left(125 \mathrm{MHz}, 323 \mathrm{~K}, \mathrm{MeCN}-d_{3}\right): \delta=167.2(\mathrm{~d}, J=21.9 \mathrm{~Hz})$, 156.7 (br), 156.3, 146.8, 139.7, 138.3, 136.0, 135.6, 129.5, 129.2, 129.0, 128.8 (br), 128.2, 127.3, 120.2, 89.8 (d, J = 180.8 Hz), 

67.4, $57.5(\mathrm{brd}, J=22.9 \mathrm{~Hz}), 46.9,25.3,15.9$ ppm; ${ }^{19} \mathrm{~F}$ NMR (376 MHz, $\left.323 \mathrm{~K}, \mathrm{MeCN}-d_{3}\right): \delta=-194.0 \mathrm{ppm}(\mathrm{d}, J=33.9 \mathrm{~Hz})$; IR (film): $\widetilde{v}=3327$ (br), 3062, 3028, 2922, 1719, 1672, 1594, 1496, 1427, 1308, 1276, 1240, 1043, 773 cm-1; HRMS (ESI): m/z calculated for $\mathrm{C}_{25} \mathrm{H}_{25} \mathrm{FN}_{3} \mathrm{O}_{3} \mathrm{~S}[\mathrm{M}+\mathrm{H}]^{+}: 466.1595$ found: $466.1590 ;[\alpha]_{D}^{26}=-25.6$ (c=0.80, $\mathrm{CHCl}_{3}, 90 \%$ ee sample); HPLC analysis (CHIRALPAK IA $(\phi=0.46 \mathrm{~cm} \times 25 \mathrm{~cm}), 2$-propanol $/ n$-hexane $=1 / 4$, flow rate $=1.0 \mathrm{~mL} / \mathrm{min}$, detection at 254 $\mathrm{nm}, \mathrm{t}=33.3 \mathrm{~min}$ (major), $71.7 \mathrm{~min}($ minor)):
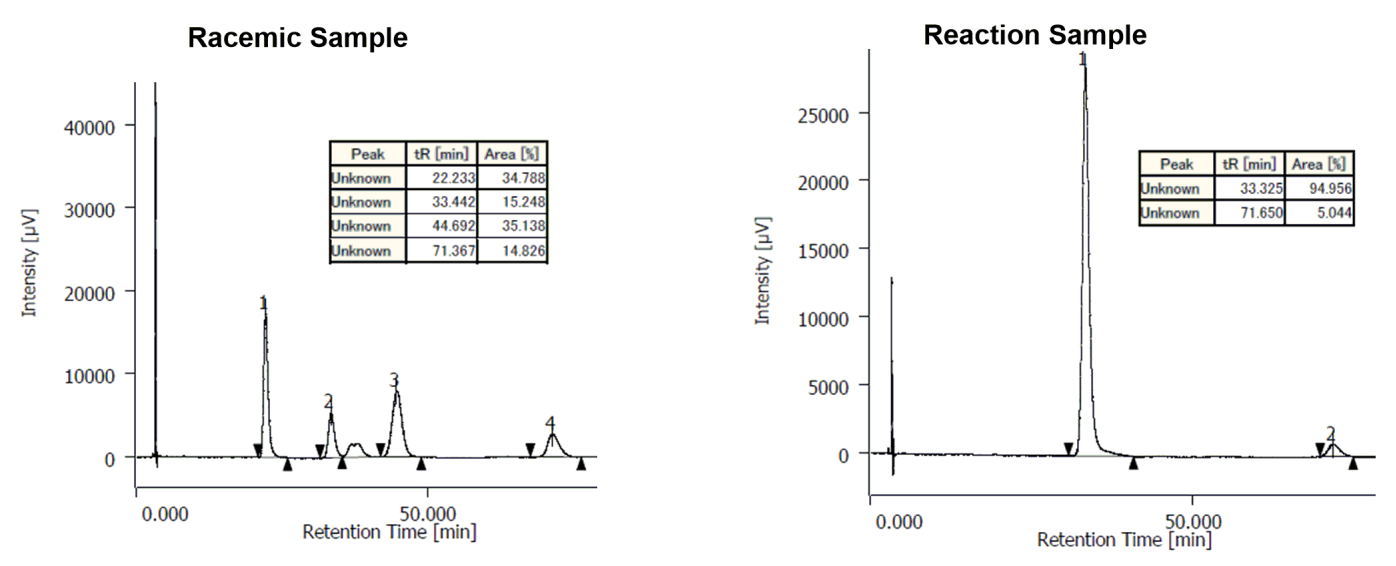

\section{Benzyl ((1S,2S)-3-(2,3-dihydro-1H-pyrrolo[2,3-b]pyridin-1-yl)2-fluoro-3-oxo-1-(thiophen-3-yl)propyl)carbamate}

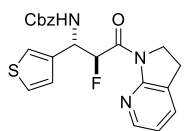
(15za).

The reaction was performed according to procedure D using $\mathrm{N}-\mathrm{Cbz}$ imine $2 \mathrm{za}$ (49.1 $\mathrm{mg}$ ). After $48 \mathrm{~h}$ the conversion was determined to be $80 \%$ and the initial diastereomeric ratio of the product to be 9:1 (anti:syn). 28.0 mg $(66 \%)$ of the desired pure diastereomer 15za were obtained after purification by flash column chromatography (hexane/ethyl acetate, 1:2).

White solid, m. p.: $145-147^{\circ} \mathrm{C} ;{ }^{1} \mathrm{H}$ NMR $\left(400 \mathrm{MHz}, 323 \mathrm{~K}, \mathrm{MeCN}-d_{3}\right): \delta=8.08(\mathrm{~d}, J=5.0 \mathrm{~Hz}, 1 \mathrm{H}), 7.59$ (ddd, $J=7.4,2.7$, $1.3 \mathrm{~Hz}, 1 \mathrm{H}), 7.37-7.26(\mathrm{~m}, 7 \mathrm{H}), 7.14-7.13(\mathrm{~m}, 1 \mathrm{H}), 7.00(\mathrm{dd}, J=7.4,5.1 \mathrm{~Hz}, 1 \mathrm{H}), 6.76(\mathrm{dd}, J=48.5,6.1 \mathrm{~Hz}, 1 \mathrm{H}), 6.56(\mathrm{brs}$, $1 \mathrm{H}), 5.44(\mathrm{ddd}, J=17.6,9.4,6.1 \mathrm{~Hz}, 1 \mathrm{H}), 5.07(\mathrm{~d}, J=12.6 \mathrm{~Hz}, 1 \mathrm{H}), 5.01(\mathrm{~d}, J=12.6 \mathrm{~Hz}, 1 \mathrm{H}), 4.08(\mathrm{ddd}, J=12.0,10.3,6.6 \mathrm{~Hz}$, 1H), $3.87(\mathrm{ddd}, J=12.0,10.4,6.5 \mathrm{~Hz}, 1 \mathrm{H}), 3.13-2.96 \mathrm{ppm}(\mathrm{m}, 2 \mathrm{H}) ;{ }^{13} \mathrm{C} \mathrm{NMR}\left(125 \mathrm{MHz}, 323 \mathrm{~K}, \mathrm{MeCN}-d_{3}\right): \delta=167.1(\mathrm{~d}, J=$ $21.8 \mathrm{~Hz}), 156.6$ (br), 156.3, 146.9, 140.2, 138.3, 135.6, 129.6, 129.0, 128.8, 128.1, 128.0(5), 127.1, 124.2 (d, J= $1.1 \mathrm{~Hz}), 120.2$, $90.0(\mathrm{~d}, J=180.7 \mathrm{~Hz}), 67.4,53.8$ (d, $J=24.3 \mathrm{~Hz}), 46.9,25.2$ ppm; ${ }^{19} \mathrm{~F}$ NMR (376 MHz, $\left.323 \mathrm{~K}, \mathrm{MeCN}-d_{3}\right): \delta=-194.0$ ppm $(\mathrm{d}$, $J=37.6 \mathrm{~Hz}$ ); IR (film): $\widetilde{v}=3324$ (br), 3093, 3062, 3032, 2952, 2918, 1718, 1671, 1594, 1508, 1428, 1308, 1239, 1039, 774 cm-1; HRMS (ESI): $m / z$ calculated for $\mathrm{C}_{22} \mathrm{H}_{21} \mathrm{FN}_{3} \mathrm{O}_{3} \mathrm{~S}[\mathrm{M}+\mathrm{H}]^{+}: 426.1282$, found: $426.1277 ;[\alpha]_{D}^{26}=-69.5\left(c=0.50, \mathrm{CHCl}_{3}, 92 \%\right.$ ee sample); HPLC analysis (CHIRALPAK IA $(\phi=0.46 \mathrm{~cm} \times 25 \mathrm{~cm}), 2$-propanol $/ n$-hexane $=1 / 4$, flow rate $=1.0 \mathrm{~mL} / \mathrm{min}$, detection at $254 \mathrm{~nm}, \mathrm{t}_{\mathrm{R}}=30.8 \mathrm{~min}$ (major), $47.1 \mathrm{~min}$ (minor)): 

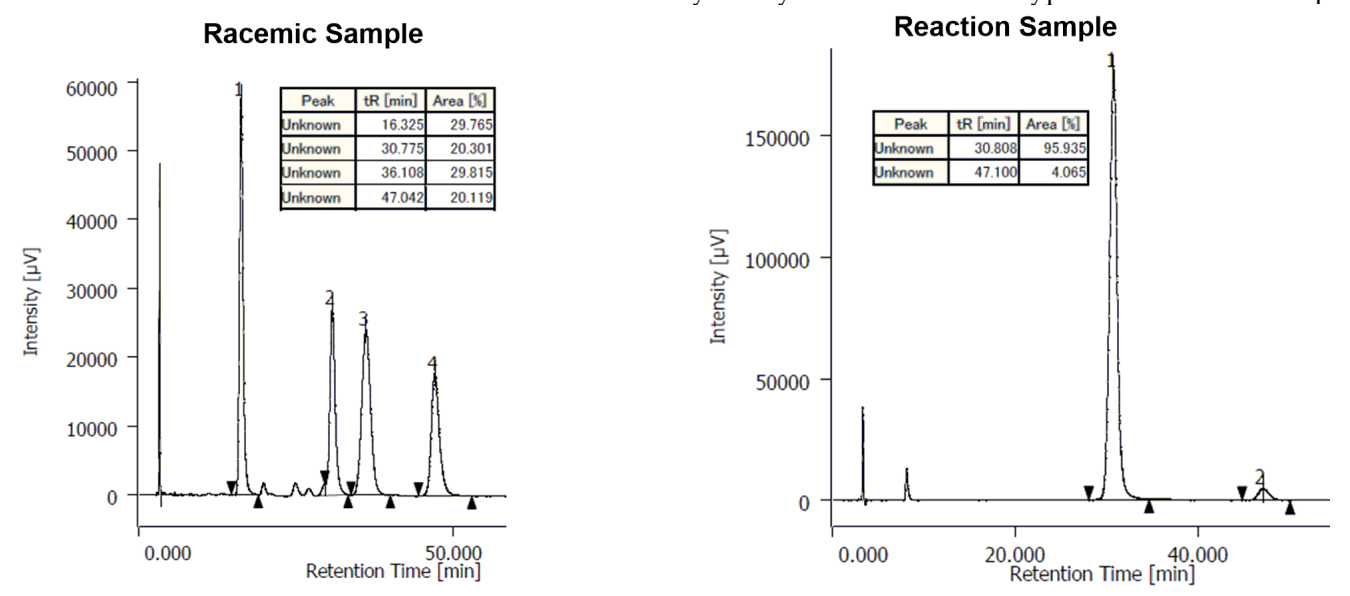

\section{4-8. Transformation of the Mannich products.}

tert-Butyl ((1S,2S)-3,3,3-trifluoro-2-(hydroxymethyl)-1-(4-methoxyphenyl)propyl)carbamate (4).<smiles>COc1ccc(C(N)C(C)CO)c(O)c1</smiles>

A flame-dried $50 \mathrm{~mL}$ flask was charged with amide $3 \mathrm{~h}$ (46.5 mg, $0.1 \mathrm{mmol}, 1.0$ equiv.). After evacuating for $15 \mathrm{~min}$, the tube was backfilled with Ar and anhydrous diethyl ether (3 mL) was added.

The resulting clear colorless solution was cooled to $-84^{\circ} \mathrm{C}$ using an ethyl acetate/liquid $\mathrm{N}_{2}$ cooling bath and a solution of $\mathrm{LiAlH}_{4}(1.0 \mathrm{M}$ in Et2 $\mathrm{O}, 0.3 \mathrm{~mL}, 0.3 \mathrm{mmol}, 3.0$ equiv.) was added. The reaction mixture was gradually warmed to $-45{ }^{\circ} \mathrm{C}$ over $90 \mathrm{~min}$ before it was cooled to $-84^{\circ} \mathrm{C}$, diluted with anhydrous diethyl ether $(10 \mathrm{~mL})$ and carefully quenched under vigorous stirring by the addition of citric acid (1.0 M in THF, $1 \mathrm{~mL})$. Caution: To suppress substantial epimerization of the aldehyde, the temperature inside the flask must be kept constant during the quenching process. The mixture was poured on aqueous citric acid $(0.5 \mathrm{M})$ and extracted three times with diethyl ether. The combined organic extracts were dried over $\mathrm{Na}_{2} \mathrm{SO}_{4}$, filtered and evaporated to give the crude aldehyde, which was directly used in the following step. ${ }^{1} \mathrm{H}$ NMR analysis of the aldehyde indicated typically a diastereomeric ratio of $>20: 1$.

The crude aldehyde was dissolved in anhydrous diethyl ether $(2 \mathrm{~mL})$ and anhydrous methanol $(4 \mathrm{~mL})$. At $-20{ }^{\circ} \mathrm{C}$ $\mathrm{NaBH}_{4}(37.4 \mathrm{mg}, 1.0 \mathrm{mmol}, 10$ equiv.) was added portionwise to the clear solution, and the reaction mixture was stirred at $0^{\circ} \mathrm{C}$ for 90 min before it was carefully quenched with aqueous $\mathrm{HCl}(1 \mathrm{M})$ and extracted three times with ethyl acetate. The combined organic extracts were dried over $\mathrm{Na}_{2} \mathrm{SO}_{4}$, filtered and evaporated to afford $26 \mathrm{mg}$ (74 \%) of the desired product as a single diastereomer after purification by flash column chromatography (hexane/ethyl acetate, 3:1). White solid, m. p.: $131-133{ }^{\circ} \mathrm{C}$; ${ }^{1} \mathrm{H}$ NMR (400 MHz, $\left.300 \mathrm{~K}, \mathrm{MeCN}-d_{3}\right): \delta=7.29-7.25$ (m, 2H), 6.93-6.89 (m, $\left.2 \mathrm{H}\right), 6.07$ (brd, $J=7.8 \mathrm{~Hz}, 1 \mathrm{H}), 5.06$ (brt, $J=8.2 \mathrm{~Hz}, 1 \mathrm{H}), 3.78(\mathrm{~s}, 3 \mathrm{H}), 3.71-3.66(\mathrm{~m}, 1 \mathrm{H}), 3.39$ (brd, $J=10.5 \mathrm{~Hz}, 1 \mathrm{H}), 3.09(\mathrm{brs}, 1 \mathrm{H})$, 2.83-2.72 (m, 1H), 1.36 ppm (s, 9H); ${ }^{13} \mathrm{C}$ NMR (100 MHz, $\left.300 \mathrm{~K}, \mathrm{MeCN}-d_{3}\right): \delta=160.1,155.8$ (br), 133.1, 129.5, 128.0 (q, $J=$ 281.2 Hz), 114.7, 79.8 (br), 58.2 (q, J = 3.5 Hz), 55.9, 52.0 (br), 50.4 (q, J = 22.7 Hz), 28.5 ppm; ${ }^{19} \mathrm{~F} \mathrm{NMR} \mathrm{(376} \mathrm{MHz,} 300 \mathrm{~K}$, MeCN-d3): $\delta=-64.3$ ppm (m); IR (film): $\widetilde{v}=3489$ (br), 3370 (br), 3007, 2978, 2932, 2904, 1685, 1613, 1530, 1515, 1390, 1368, 1347, 1298, 1243, 1159, 1120, 1110, 1088, 1032, 1016, $834 \mathrm{~cm}^{-1} ;$ HRMS (ESI): $m / z$ calculated for $\mathrm{C}_{16} \mathrm{H}_{22} \mathrm{~F}_{3} \mathrm{NO}_{4} \mathrm{Na}_{[\mathrm{M}+\mathrm{Na}}{ }^{+}$: 372.1393, found: 372.1386; $[\alpha]_{D}^{24}=-31.9\left(c=1.20, \mathrm{CHCl}_{3}, 98 \%\right.$ ee sample). 

(S)-2-((S)-((tert-Butoxycarbonyl)amino)(4-methoxyphenyl)methyl)-3,3,3-trifluoropropanoic acid (5).<smiles>COc1ccc(C(NC(=O)C(C)C(F)(F)F)C(=O)O)cc1</smiles>

A flame-dried $50 \mathrm{~mL}$ flask was charged with amide $3 \mathrm{~h}(46.5 \mathrm{mg}, 0.1 \mathrm{mmol}, 1.0$ equiv.). After evacuating for $15 \mathrm{~min}$, the tube was backfilled with Ar and anhydrous diethyl ether ( $3 \mathrm{~mL})$ was added. of $\mathrm{LiAlH}_{4}\left(1.0 \mathrm{M}\right.$ in $\mathrm{Et}_{2} \mathrm{O}, 0.3 \mathrm{~mL}, 0.3 \mathrm{mmol}, 3.0$ equiv.) was added. The reaction mixture was gradually warmed to $-45{ }^{\circ} \mathrm{C}$ over $90 \mathrm{~min}$ before it was cooled to $-84{ }^{\circ} \mathrm{C}$, diluted with anhydrous diethyl ether $(10 \mathrm{~mL})$ and carefully quenched under vigorous stirring by the addition of citric acid (1.0 M in THF, $1 \mathrm{~mL})$. Caution: To suppress substantial epimerization of the a-stereocenter, the temperature inside the flask must be kept constant during the quenching process. The mixture was poured on aqueous citric acid $(0.5 \mathrm{M})$ and extracted three times with diethyl ether. The combined organic extracts were dried over $\mathrm{Na}_{2} \mathrm{SO}_{4}$, filtered and evaporated to give the crude aldehyde, which was directly used in the following step. ${ }^{1} \mathrm{H}$ NMR analysis of the aldehyde indicated typically a diastereomeric ratio of $>20: 1$. The crude aldehyde was dissolved at $0{ }^{\circ} \mathrm{C}$ in water $(0.8 \mathrm{~mL})$ and tert-butanol $(1.0 \mathrm{~mL}$, reagent grade) under an atmosphere of air. $\mathrm{NaH}_{2} \mathrm{PO}_{4} \cdot 2 \mathrm{H}_{2} \mathrm{O}(46.8 \mathrm{mg}, 0.3 \mathrm{mmol}$, 3.0 equiv.), 2-methylbut-2-ene (0.2 mL, $1.9 \mathrm{mmol}, 19$ equiv.), and $\mathrm{NaClO}_{2}$ (31.7 mg, $0.35 \mathrm{mmol}, 3.5$ equiv.) were subsequently added to the clear solution. After stirring for $30 \mathrm{~min}$ at the same temperature, the reaction mixture was stirred for a further $5 \mathrm{~h}$ at ambient temperature. Aqueous $\mathrm{NaOH}(1 \mathrm{M})$ was added and the mixture was extracted five times with dichloromethane. The aqueous phase was acidified with aqueous $\mathrm{HCl}(6 \mathrm{M})$ and extracted five times with dichloromethane. These combined organic extracts were washed with saturated aqueous $\mathrm{KHSO}_{4}$ solution, dried over $\mathrm{Na}_{2} \mathrm{SO}_{4}$, filtered and evaporated to afford $24 \mathrm{mg}(66 \%)$ of the desired product as an inseparable mixture of diastereomers ( $\mathrm{dr}>10: 1)$. All NMR data are given for the major diastereomer.

White solid, m. p.: $169-171{ }^{\circ} \mathrm{C}$; ${ }^{1} \mathrm{H}$ NMR $\left(400 \mathrm{MHz}, 300 \mathrm{~K}, \mathrm{MeCN}-d_{3}\right): \delta=7.30-7.25(\mathrm{~m}, 2 \mathrm{H}), 6.91-6.88(\mathrm{~m}, 2 \mathrm{H}), 5.98$ (brd, $J=7.1 \mathrm{~Hz}, 1 \mathrm{H}), 5.34(\mathrm{brs}, 1 \mathrm{H}$ and residual water), $5.14(\mathrm{brt}, J=9.8 \mathrm{~Hz}, 1 \mathrm{H}), 3.78(\mathrm{~s}, 3 \mathrm{H}), 3.82-3.74(\mathrm{~m}, 1 \mathrm{H}), 1.36 \mathrm{ppm}(\mathrm{s}$, 9H); ${ }^{13} \mathrm{C}$ NMR (100 MHz, $\left.300 \mathrm{~K}, \mathrm{MeCN}-d_{3}\right): \delta=166.9$ (q, J = $\left.2.9 \mathrm{~Hz}\right), 160.5,155.5(\mathrm{br}), 131.9,129.4,125.2(\mathrm{q}, J=278.3 \mathrm{~Hz})$, 114.9, 80.1 (br), 55.9, 55.6 (q, J = 25.2 Hz), 52.8 (br), 28.4 ppm; ${ }^{19} \mathrm{~F}$ NMR (376 MHz, $\left.300 \mathrm{~K}, \mathrm{MeCN}-d_{3}\right): \delta=-65.0 \mathrm{ppm}(\mathrm{s}) ; \mathrm{IR}$ (film): $\widetilde{v}=3355$ (br), 2979, 2935, 1724, 1680, 1614, 1514, 1426, 1371, 1249, 1158, 1111, 1027, 832, $768 \mathrm{~cm}^{-1}$; HRMS (ESI): m/z calculated for $\mathrm{C}_{16} \mathrm{H}_{20} \mathrm{~F}_{3} \mathrm{NO}_{5} \mathrm{Na}[\mathrm{M}+\mathrm{Na}]^{+}: 386.1159$, found: 386.1176; $[\alpha]_{D}^{24}=-89.5$ ( $c=0.85, \mathrm{MeOH}, \mathrm{dr}>10: 1$ sample).

\section{tert-Butyl ((1R,2R)-2-benzoyl-2,3,3,3-tetrafluoro-1-(furan-3-yl)propyl)carbamate (7).}

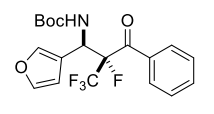

A flame-dried $20 \mathrm{~mL}$ test tube was charged with amide $6 \mathrm{p}$ (44.3 mg, $0.1 \mathrm{mmol}, 1.0$ equiv.). After evacuating for $15 \mathrm{~min}$, the tube was backfilled with Ar and anhydrous diethyl ether $(1.0 \mathrm{~mL})$ was added.

Subsequently, phenylmagnesium bromide ( $3.0 \mathrm{M}$ in Et2 $\mathrm{O}, 0.17 \mathrm{~mL}, 0.5 \mathrm{mmol}, 5.0$ equiv.) was added at $0{ }^{\circ} \mathrm{C}$. The reaction mixture was stirred at ambient temperature for $150 \mathrm{~min}$ before it was cooled to $0{ }^{\circ} \mathrm{C}$ and quenched by the addition of saturated aqueous $\mathrm{NH}_{4} \mathrm{Cl}$ solution. The mixture was diluted with water and extracted three times with ethyl acetate; the combined organic extracts were dried over $\mathrm{Na}_{2} \mathrm{SO}_{4}$, filtered and evaporated to afford $35 \mathrm{mg}(88 \%)$ of the pure desired product 7 after purification by flash column chromatography (hexane/ethyl acetate, 10:1).

Clear colorless oil; ${ }^{1} \mathrm{H}$ NMR (400 MHz, $343 \mathrm{~K}$, DMSO- $\left.d_{6}\right): \delta=7.77$ (brs, 1H), 7.64-7.54 (m, 4H), 7.48-7.44 (m, 3H), 6.53 (s, $1 \mathrm{H}), 5.74(\mathrm{dd}, J=30.5,10.2 \mathrm{~Hz}, 1 \mathrm{H}), 1.41 \mathrm{ppm}(\mathrm{s}, 9 \mathrm{H}) ;{ }^{13} \mathrm{C}$ NMR $\left(125 \mathrm{MHz}, 343 \mathrm{~K}, \mathrm{DMSO}-d_{6}\right): \delta=193.5(\mathrm{~d}, J=24.6 \mathrm{~Hz})$, 

154.2 (br), 143.2, 141.4, 134.1, 133.7, 128.3, 128.2(8), 121.1 (qd, J = 287.8, 30.2 Hz), 119.2, 110.3, 99.8 (dq, J = 211.1, $27.6 \mathrm{~Hz}$ ), 78.8, 48.3 (brd, $J=15.9 \mathrm{~Hz}), 27.8$ ppm; ${ }^{19} \mathrm{~F}$ NMR (376 MHz, $\left.343 \mathrm{~K}, \mathrm{DMSO}-d_{6}\right): \delta=-71.7$ (s, 3F), -181.2 ppm (s, 1F); IR (film): $\widetilde{v}=3350$ (br), 3147, 2980, 2931, 1709, 1598, 1504, 1369, 1313, 1266, 1209, 1164, 1023, 876 cm-1; HRMS (ESI): m/z calculated for $\mathrm{C}_{19} \mathrm{H}_{19} \mathrm{~F}_{4} \mathrm{NO}_{4} \mathrm{Na}[\mathrm{M}+\mathrm{Na}]^{+}: 424.1148$, found: $424.1141 ;[\alpha]_{D}^{25}=-17.6\left(c=0.85, \mathrm{CHCl}_{3}\right)$.

\section{tert-Butyl ((1R,2R)-2,3,3,3-tetrafluoro-2-formyl-1-(furan-3-yl)propyl)carbamate (8).}

BochN A flame-dried $20 \mathrm{~mL}$ flask was charged with amide 6 p (50.0 mg, $0.11 \mathrm{mmol}, 1.0$ equiv.). After evacuating for $15 \mathrm{~min}$, the tube was backfilled with Ar and anhydrous diethyl ether $(3.0 \mathrm{~mL})$ was added. The resulting clear colorless solution was cooled to $-70{ }^{\circ} \mathrm{C}$ and a solution of $\mathrm{LiAlH}_{4}(1.0 \mathrm{M}$ in Et $2 \mathrm{O}, 0.25 \mathrm{~mL}, 0.25 \mathrm{mmol}, 2.2$ equiv.) was slowly added. The reaction mixture was stirred for $15 \mathrm{~min}$ before it was cooled to $-84{ }^{\circ} \mathrm{C}$ using an ethyl acetate/liquid $\mathrm{N}_{2}$ cooling bath, diluted with anhydrous diethyl ether $(5 \mathrm{~mL})$ and carefully quenched under vigorous stirring by the addition of citric acid (1.0 M in THF, $1 \mathrm{~mL}$ ). Afterwards, the mixture was poured on aqueous citric acid $(0.5 \mathrm{M})$ and extracted three times with diethyl ether. The combined organic extracts were dried over $\mathrm{Na}_{2} \mathrm{SO}_{4}$, filtered and evaporated to give the crude aldehyde, which was directly purified by quickly passing through a short pad of silica between two thin layers of Celite ${ }^{\circledR}$ (hexane/ethyl acetate, 5:1). After evaporation, $27.0 \mathrm{mg}$ (74\%) of the pure desired product 8 were isolated as a single diastereomer.

White solid, m. p.: $97-99{ }^{\circ} \mathrm{C} ;{ }^{1} \mathrm{H}$ NMR $\left(500 \mathrm{MHz}, 300 \mathrm{~K}, \mathrm{MeCN}-d_{3}\right): \delta=9.46(\mathrm{dq}, J=7.1,1.7 \mathrm{~Hz}, 1 \mathrm{H}), 7.54$ (s, $\left.1 \mathrm{H}\right), 7.49$ (t, $J$ $=1.6 \mathrm{~Hz}, 1 \mathrm{H}), 6.47(\mathrm{~s}, 1 \mathrm{H}), 6.24(\mathrm{brd}, J=6.9 \mathrm{~Hz}, 1 \mathrm{H}), 5.65(\mathrm{brdd}, J=29.8,9.7 \mathrm{~Hz}, 1 \mathrm{H}), 1.41 \mathrm{ppm}(\mathrm{s}, 9 \mathrm{H})$; ${ }^{13} \mathrm{C} \mathrm{NMR}(125$ $\left.\mathrm{MHz}, 300 \mathrm{~K}, \mathrm{MeCN}-d_{3}\right): \delta=192.6$ (d, $\left.J=30.9 \mathrm{~Hz}\right), 155.5(\mathrm{br}), 145.3,142.5$ (d, J = 1.3 Hz), 121.9 (qd, J = 286.1, 29.3 Hz), 120.1, 110.7, $97.6(\mathrm{dq}, J=200.8,28.7 \mathrm{~Hz}), 80.8,47.3(\mathrm{brd}, J=18.4 \mathrm{~Hz}), 28.3$ ppm; ${ }^{19} \mathrm{~F}$ NMR $\left(376 \mathrm{MHz}, 300 \mathrm{~K}, \mathrm{MeCN}-d_{3}\right): \delta=$ -74.0 (s, 3F), -193.9 ppm (s, 1F); IR (KBr): $\widetilde{v}=3372,2983,2939,2877,1750,1697,1523,1394,1369,1326,1280,1253,1205$, 1172, 1054, 1024, 875, $798 \mathrm{~cm}^{-1}$; HRMS (ESI): $m / z$ calculated for $\mathrm{C}_{13} \mathrm{H}_{15} \mathrm{~F}_{4} \mathrm{NO}_{4} \mathrm{Na}[\mathrm{M}+\mathrm{Na}]^{+}: 348.0829$, found: 348.0828; $[\alpha]_{D}^{26}=-13.6\left(c=0.30, \mathrm{CHCl}_{3}\right)$.

\section{(R)-2-((R)-amino(furan-3-yl)methyl)-2,3,3,3-tetrafluoropropanoic acid hydrochloride (9).}

A $20 \mathrm{~mL}$ test tube was charged with amide $6 \mathrm{p}$ (50.0 mg, $0.11 \mathrm{mmol}, 1.0$ equiv.) under air atmosphere. THF $\left(2.0 \mathrm{~mL}\right.$, reagent grade) and aqueous $\mathrm{HCl}$ solution $\left(2.0 \mathrm{~mL}, 6 \mathrm{M}\right.$ in $\left.\mathrm{H}_{2} \mathrm{O}\right)$ were subsequently added. The reaction mixture was stirred at $60{ }^{\circ} \mathrm{C}$ for $9 \mathrm{~h}$ before it was cooled to ambient temperature, evaporated and directly purified by flash column chromatography using basic Wakogel ${ }^{\circledR 5} \mathrm{NH}_{2}$ (dichloromethane/methanol, 4:1; then: $2 \mathrm{M} \mathrm{HCl}$ solution in methanol) to afford $30 \mathrm{mg}(97 \%)$ of the pure desired product 9.

White solid, decomposes above $227^{\circ} \mathrm{C} ;{ }^{1} \mathrm{H}$ NMR (600 MHz, $\left.298 \mathrm{~K}, \mathrm{D}_{2} \mathrm{O}\right): \delta=7.68(\mathrm{~s}, 1 \mathrm{H}), 7.55$ (s, $\left.1 \mathrm{H}\right), 6.55$ (s, $\left.1 \mathrm{H}\right), 5.29$ $\operatorname{ppm}(\mathrm{d}, J=26.5 \mathrm{~Hz}, 1 \mathrm{H}) ;{ }^{13} \mathrm{C}$ NMR $\left(150 \mathrm{MHz}, 298 \mathrm{~K}, \mathrm{D}_{2} \mathrm{O}\right): \delta=166.0(\mathrm{~d}, J=20.2 \mathrm{~Hz}), 145.3,143.6,122.1$ (qd, $J=285.2,30.3$ $\mathrm{Hz}), 116.0,109.3(\mathrm{~d}, J=2.9 \mathrm{~Hz}), 93.8(\mathrm{dq}, J=207.9,29.4 \mathrm{~Hz}), 48.2$ ppm (d, $J=20.2 \mathrm{~Hz}) ;{ }^{19} \mathrm{~F} \mathrm{NMR}(376 \mathrm{MHz}, 300 \mathrm{~K}, \mathrm{D} 2 \mathrm{O}): \delta$ $=-74.2(\mathrm{~s}, 3 \mathrm{~F}),-178.5$ ppm (s, 1F); IR (KBr): $\widetilde{v}=3000$ (br), 2844 (br), 1770, 1670, 1595, 1564, 1517, 1391, 1281, 1244, 1223, 1194, 1176, 1105, 1083, 1015, $876 \mathrm{~cm}^{-1}$; HRMS (ESI): $m / z$ calculated for $\mathrm{C}_{8} \mathrm{H}_{8} \mathrm{~F}_{4} \mathrm{NO}_{3}[\mathrm{M}-\mathrm{HCl}+\mathrm{H}]^{+}: 242.0435$, found: 242.0431; $[\alpha]_{D}^{25}=+10.1(c=0.70, \mathrm{MeOH})$. 

tert-Butyl ((1R,2R)-2,3,3,3-tetrafluoro-1-(furan-3-yl)-2-(hydroxymethyl)propyl)carbamate (10).

(O)

To a solution of lithium diisopropylamide (1.13 M in n-hexane/THF (5.4:1), $0.345 \mathrm{~mL}, 0.39 \mathrm{mmol}, 3.9$ equiv.) in anhydrous THF ( $0.5 \mathrm{~mL})$ was added borane-ammonia complex (12.3 mg, $0.40 \mathrm{mmol}, 4.0$ equiv.)

at $0{ }^{\circ} \mathrm{C}$. After stirring for $10 \mathrm{~min}$ at the same temperature, the reaction mixture was stirred at room temperature for another $10 \mathrm{~min}$ before it was cooled back to $0{ }^{\circ} \mathrm{C}$. A solution of amide $6 \mathbf{p}(44.3 \mathrm{mg}, 0.1 \mathrm{mmol}, 1.0 \mathrm{equiv}$.) in anhydrous THF (0.5 mL) was subsequently added. After stirring for $30 \mathrm{~min}$, the reaction mixture was warmed to ambient temperature and stirred for another $30 \mathrm{~min}$ before it was finally quenched by the addition of saturated aqueous $\mathrm{NH}_{4} \mathrm{Cl}$ solution. The mixture was diluted with water and extracted three times with ethyl acetate; the combined organic extracts were treated with aqueous $\mathrm{NaOH}(1 \mathrm{M})$ solution and stirred for $30 \mathrm{~min}$. Then, the layers were separated and the aqueous layer was extracted twice with ethyl acetate; the combined organic extracts were dried over $\mathrm{Na}_{2} \mathrm{SO}_{4}$, filtered and evaporated to afford $26 \mathrm{mg}(81 \%)$ of the pure desired product 10 after purification by flash column chromatography (hexane/ethyl acetate, 5:1).

White solid, m. p.: $116-118{ }^{\circ} \mathrm{C} ;{ }^{1} \mathrm{H}$ NMR $\left(400 \mathrm{MHz}, 323 \mathrm{~K}, \mathrm{MeCN}-d_{3}\right): \delta=7.56-7.55(\mathrm{~m}, 1 \mathrm{H}), 7.49(\mathrm{t}, J=1.7 \mathrm{~Hz}, 1 \mathrm{H})$, 6.53-6.51 (m, 1H), 6.00 (brs, 1H), 5.40 (dd, J = 20.3, $10.2 \mathrm{~Hz}, 1 \mathrm{H}), 3.89$ (ddd, J = 17.2, 12.1, $5.5 \mathrm{~Hz}, 1 \mathrm{H}), 3.71-3.63(\mathrm{~m}, 1 \mathrm{H})$, $3.57(\mathrm{t}, J=5.8 \mathrm{~Hz}, 1 \mathrm{H}), 1.41 \mathrm{ppm}(\mathrm{s}, 9 \mathrm{H}) ;{ }^{13} \mathrm{C}$ NMR $\left(125 \mathrm{MHz}, 323 \mathrm{~K}, \mathrm{MeCN}-d_{3}\right): \delta=156.2(\mathrm{br}), 144.8,142.6,124.6$ (qd, $J=$ 286.7, $28.3 \mathrm{~Hz}), 122.1,111.0$ (d, J = 1.6 Hz), 96.3 (dq, $J=192.0,27.0 \mathrm{~Hz}), 80.7,61.1$ (dq, $J=26.7,1.6 \mathrm{~Hz}), 48.0$ (brd, $J=15.7$ Hz), 28.7 ppm; ${ }^{19} \mathrm{~F}$ NMR (376 MHz, $\left.323 \mathrm{~K}, \mathrm{MeCN}-d_{3}\right): \delta=-74.9$ (s, 3F), -183.5 ppm (s, 1F); IR (film): $\widetilde{v}=3309$ (br), 3149 , 2982, 2941, 1677, 1548, 1507, 1369, 1332, 1288, 1258, 1206, 1171, 1111, 1110, 1026, 1007, 876, $787 \mathrm{~cm}^{-1}$; HRMS (ESI): m/z calculated for $\mathrm{C}_{13} \mathrm{H}_{17} \mathrm{~F}_{4} \mathrm{NO}_{4} \mathrm{Na}[\mathrm{M}+\mathrm{Na}]^{+}: 350.0986$, found: 350.0988; $[\alpha]_{D}^{26}=-4.7\left(c=1.00, \mathrm{CHCl}_{3}\right)$.

\section{tert-Butyl ((1R-2R)-2-(benzylcarbamoyl)-2,3,3,3-tetrafluoro-1-(furan-3-yl)propyl)carbamate (11).}

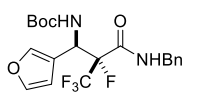

A $20 \mathrm{~mL}$ test tube was charged with lanthanum(III) trifluoromethanesulfonate $(58.6 \mathrm{mg}, 0.1 \mathrm{mmol}, 1.0$ equiv.) and flame dried under vacuum for $10 \mathrm{~min}$. After the flask cooled down to ambient temperature, it was backfilled with Ar and subsequently charged with amide $6 \mathbf{p}(44.3 \mathrm{mg}, 0.1 \mathrm{mmol}, 1.0$ equiv.) and anhydrous THF $(2.0 \mathrm{~mL}$ ). After stirring for $10 \mathrm{~min}$ at ambient temperature, benzylamine ( $43.6 \mu \mathrm{L}, 0.4 \mathrm{mmol}, 4.0$ equiv.) was added to the reaction mixture. The reaction mixture was stirred for $90 \mathrm{~min}$ before it was quenched by the addition of saturated aqueous $\mathrm{NH}_{4} \mathrm{Cl}$ solution and extracted three times with ethyl acetate. The combined organic extracts were dried over $\mathrm{Na}_{2} \mathrm{SO}_{4}$, filtered and evaporated to afford $36 \mathrm{mg}(84 \%)$ of the pure desired product 11 after purification by flash column chromatography (hexane/ethyl acetate, 5:1).

White solid, m. p.: $213-215^{\circ} \mathrm{C}$; ${ }^{1} \mathrm{H}$ NMR $\left(400 \mathrm{MHz}, 343 \mathrm{~K}, \mathrm{DMSO}-d_{6}\right): \delta=8.89(\mathrm{t}, J=5.6 \mathrm{~Hz}, 1 \mathrm{H}), 7.55-7.50(\mathrm{~m}, 3 \mathrm{H})$, 7.25-7.17 (m, 3H), 6.97-6.95 (m, 2H), $6.50(\mathrm{t}, J=1.3 \mathrm{~Hz}, 1 \mathrm{H}), 5.60(\mathrm{dd}, J=31.7,10.2 \mathrm{~Hz}, 1 \mathrm{H}), 4.26(\mathrm{dd}, J=15.0,6.5 \mathrm{~Hz}, 1 \mathrm{H})$, $4.13(\mathrm{dd}, J=15.1,5.7 \mathrm{~Hz}, 1 \mathrm{H}), 1.39 \mathrm{ppm}(\mathrm{s}, 9 \mathrm{H}) ;{ }^{13} \mathrm{C}$ NMR $\left(125 \mathrm{MHz}, 343 \mathrm{~K}, \mathrm{DMSO}-d_{6}\right): \delta=162.2(\mathrm{~d}, J=20.0 \mathrm{~Hz}), 154.2$ (br), 142.6, 141.0, 137.7, 127.7, 126.5, 126.4, 121.3 (qd, $J=286.8,29.8 \mathrm{~Hz}), 119.5,110.3,95.6$ (dq, $J=212.0,27.9 \mathrm{~Hz}), 78.5$, 47.1 (brd, $J=15.5 \mathrm{~Hz}), 41.8,27.8$ ppm; ${ }^{19} \mathrm{~F}$ NMR (376 MHz, $\left.343 \mathrm{~K}, \mathrm{DMSO}-d_{6}\right): \delta=-72.6$ (s, 3F), $-185.4 \mathrm{ppm}(\mathrm{s}, 1 \mathrm{~F}) ; \mathrm{IR}$ (film): $\widetilde{v}=3359,2983,2925,2852,1693,1675,1524,1368,1318,1279,1252,1202,1157,1141,1013,874 \mathrm{~cm}^{-1}$; HRMS (ESI): $m / z$ calculated for $\mathrm{C}_{20} \mathrm{H}_{22} \mathrm{~F}_{4} \mathrm{~N}_{2} \mathrm{O}_{4} \mathrm{Na}[\mathrm{M}+\mathrm{Na}]^{+}: 453.1413$, found: $453.1403 ;[\alpha]_{D}^{p 5}=-9.9\left(c=0.75, \mathrm{CHCl}_{3}\right)$. 

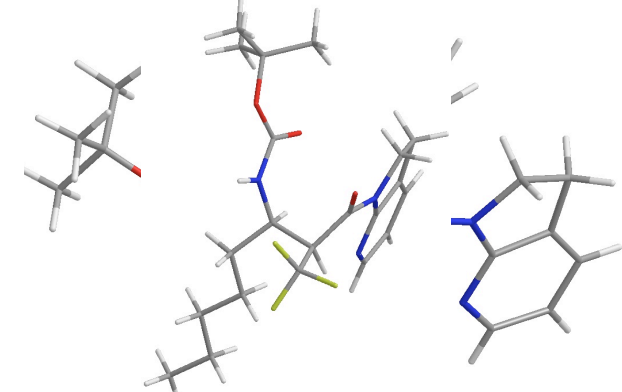
and Relative Configuration of the Mannich Products

The ausulute anu relative configurations of the other Mannich products $\mathbf{3} \mathbf{b}-\mathbf{3} \mathbf{q}$ and $\mathbf{3 r}$, $\mathbf{3} \mathbf{t}$ were deduced by analogy.

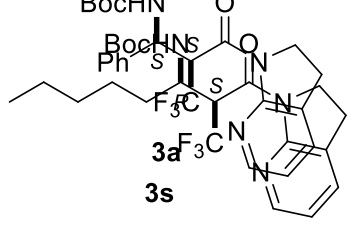

igure S4. The structure of $3 \mathbf{a}$ in the

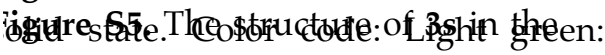

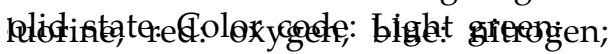

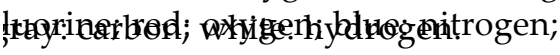
;ray: carbon; white: hydrogen.
Table S4. Selected Crystallographic Data of 3a

\begin{tabular}{c|c}
\hline & 3a \\
\hline molecular formula & $\mathrm{C}_{22} \mathrm{H}_{24} \mathrm{~F}_{3} \mathrm{~N}_{3} \mathrm{O}_{3}$ \\
formula weight & 435.45 \\
crystal color, habit & colorless, needle \\
crystal system & orthorhombic \\
space group & $P 2{ }_{12121}$ \\
cell constants: & \\
$a(\AA)$ & $10.1405(2)$ \\
$b(\AA)$ & $11.5825(2)$ \\
$c(\AA)$ & $18.6098(4)$ \\
$V(\AA)$ & $2185.78(7)$ \\
$Z$ & 4 \\
$\rho_{\text {calcd. }}\left(\mathrm{g} \cdot \mathrm{cm}^{-3}\right)$ & 1.323 \\
$R_{1}$ & 0.0280 \\
$w R_{2}$ & 0.0597 \\
$F(000)$ & 912.00 \\
Flack parameter & $-0.03(10)$ \\
\hline &
\end{tabular}

Table S5. Selected Crystallographic Data of 3s

\begin{tabular}{c|c}
\hline & $3 \mathrm{~s}$ \\
\hline molecular formula & $\mathrm{C}_{21} \mathrm{H}_{30} \mathrm{~F}_{3} \mathrm{~N}_{3} \mathrm{O}_{3}$ \\
formula weight & 429.48 \\
crystal color, habit & colorless, platelet \\
crystal system & monoclinic \\
space group & $P 21$ \\
cell constants & \\
$a(\AA)$ & $10.6993(4)$ \\
$b(\AA)$ & $9.3222(3)$ \\
$c(\AA)$ & $11.5320(4)$ \\
$\beta(\mathrm{deg})$ & $108.387(8)$ \\
$V\left(\AA \AA^{3}\right)$ & $1091.50(8)$ \\
$Z$ & 2 \\
$\rho_{\text {calcd. }}\left(\mathrm{g} \cdot \mathrm{cm}^{-3}\right)$ & 1.307 \\
$R 1$ & 0.0396 \\
$w R_{2}$ & 0.1053 \\
$F(000)$ & 456.00 \\
Flack parameter & $0.11(10)$ \\
&
\end{tabular}

In order to determine the absolute and relative configuration of the Mannich product 6a, a single-crystal was grown by leaving a concentrated solution of $6 \mathbf{a}$ in $n$-hexane/ethyl acetate at room temperature. Single-crystal X-ray data were 

II imaging plate area detector with graphite-monochromated $\mathrm{Cu}-\mathrm{K} \alpha$ radiation.

K. All structures were solved by direct methods and refined by full matrix ions. All non-hydrogen atoms were refined anisotropically. All hydrogen atoms were pracea in stanaara caıcuratea positions, and were refined using the riding model. Refined structure and crystallographic parameters are summarized in Figure S6 and Table S6. The absolute and relative configuration of 6a was determined to be as depicted below by Flack parameter. ${ }^{11}$ CCDC 1430617 contains the supplementary crystallograpjác Fetata for 6 a.

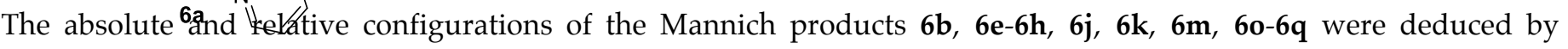
igure analog. $^{\text {qhe }}$ structure of $\mathbf{6 a}$ in the solid tate. Color code: Light green: fluorine; red: ixygen; blue: nitrogen; gray: carbon; white: ıydrogen.

Table S6. Selected Crystallographic Data of 6a

\begin{tabular}{c|c}
\hline & $\mathbf{6 a}$ \\
\hline molecular formula & $\mathrm{C}_{22} \mathrm{H}_{23} \mathrm{~F}_{4} \mathrm{~N}_{3} \mathrm{O}_{3}$ \\
formula weight & 453.44 \\
crystal color, habit & colorless, block \\
crystal system & monoclinic \\
space group & $P 21$ \\
cell constants: & \\
$a(\AA)$ & $5.36319(14)$ \\
$b(\AA)$ & $20.3350(5)$ \\
$c(\AA)$ & $9.7083(3)$ \\
$\beta($ deg $)$ & $91.135(6)$ \\
$V\left(\AA^{3}\right)$ & $1058.58(5)$ \\
$Z$ & 2 \\
$\rho_{\text {calcd. }}\left(\mathrm{g} \cdot \mathrm{cm}^{-3}\right)$ & 1.422 \\
$R 1$ & 0.0412 \\
$w R_{2}$ & 0.1191 \\
$F(000)$ & 472.00 \\
Flack parameter & $0.03(8)$
\end{tabular}

In order to determine the absolute and relative configuration of the Mannich product 12a, a single-crystal was grown by leaving a concentrated solution of 12a in $n$-hexane/ethyl acetate at room temperature. Single-crystal X-ray data were collected on a Rigaku R-AXIS RAPID II imaging plate area detector with graphite-monochromated Cu-K $\alpha$ radiation. Data collection was conducted at $93 \mathrm{~K}$. All structures were solved by direct methods and refined by full matrix least-squares against $F^{2}$ with all reflections. All non-hydrogen atoms were refined anisotropically. All hydrogen atoms were placed in standard calculated positions, and were refined using the riding model. Refined structure and crystallographic parameters are summarized in Figure S7 and Table S7. The absolute and relative configuration of 12a was determined to be as depicted below by Flack parameter. ${ }^{11}$ CCDC 1430725 contains the supplementary crystallographic data for 12a.

The absolute and relative configurations of the Mannich products $\mathbf{1 2 b}, \mathbf{1 2 c}, \mathbf{1 2 e}, \mathbf{1 2} \mathrm{g}, \mathbf{1 2 k}, \mathbf{1 2 m}, \mathbf{1 2 0}, \mathbf{1 2 s}$ as well as of 14a and $14 \mathbf{b}$ were deduced by analogy. 


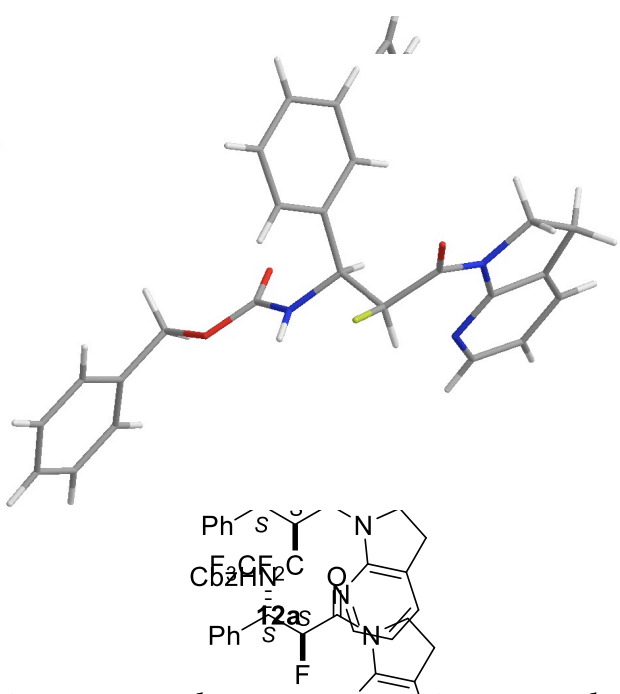

Figure S7. The st5ucture of $\mathbf{1 2 a}$ in the solid state. Color code: Light green: flugrine; redine oxygen blue: ${ }^{\text {phitrogen; }}$ gralyd carbon; white: hydrogen. color grat green: fluorine; red: oxygen; blue: nitrogen;
Supporting Information

Direct Catalytic Asymmetric Mannich-type Reaction of $\alpha$ - and $\beta$-Fluorinated Amides Table S7. Selected Crystallographic Data of 12a

\begin{tabular}{c|c}
\hline & 12a \\
\hline molecular formula & $\mathrm{C}_{23} \mathrm{H}_{24} \mathrm{~F}_{5} \mathrm{~N}_{3} \mathrm{O}_{3}$ \\
formula weight & 485.45 \\
crystal system & orthorhombic \\
space group & $P 22_{212}$ \\
cell constants: & \\
$a(\AA)$ & 11.45781 \\
$b(\AA)$ & 11.60882 \\
$c(\AA)$ & 18.71450 \\
$V\left(\AA \AA^{3}\right)$ & 2489.24660 \\
$Z$ & 4 \\
$\rho_{\text {calcd. }}\left(\mathrm{g} \cdot \mathrm{cm}^{-3}\right)$ & 1.295 \\
$R_{1}$ & 0.0549 \\
$w R_{2}$ & 0.1742 \\
$F(000)$ & 1008.00 \\
Flack parameter & $-0.10(6)$
\end{tabular}

In order to determine the absolute and relative configuration of the Mannich product 15b, a single-crystal was grown by leaving a concentrated solution of $\mathbf{1 5 b}$ in $n$-hexane/dichloromethane at room temperature. Single-crystal X-ray data were collected on a Rigaku R-AXIS RAPID II imaging plate area detector with graphite-monochromated $\mathrm{Cu}-\mathrm{K} \alpha$ radiation. Data collection was conducted at $93 \mathrm{~K}$. All structures were solved by direct methods and refined by full matrix least-squares against $F^{2}$ with all reflections. All non-hydrogen atoms were refined anisotropically. All hydrogen atoms were placed in standard calculated positions, and were refined using the riding model. Refined structure and crystallographic parameters are summarized in Figure S8 and Table S8. The absolute and relative configuration of $\mathbf{1 5 b}$ was determined to be as depicted below by Flack parameter. ${ }^{11}$ CCDC 1432081 contains the supplementary crystallographic data for $\mathbf{1 5 b}$.

The absolute and relative configurations of the Mannich products $\mathbf{1 5 u - 1 5 z a}$ were deduced by analogy.

Table S8. Selected Crystallographic Data of $\mathbf{1 5 b}$

\begin{tabular}{c|c}
\hline & $\mathbf{1 5 b}$ \\
\hline molecular formula & $\mathrm{C}_{24} \mathrm{H}_{22} \mathrm{FN}_{3} \mathrm{O}_{3} \cdot \mathrm{CH}_{2} \mathrm{Cl}_{2}$ \\
formula weight & 504.39 \\
crystal color, habit & colorless, needle \\
crystal system & monoclinic \\
space group & $\mathrm{C} 2$ \\
cell constants: & \\
$a(\AA)$ & 28.55192 \\
$b(\AA)$ & 5.29437 \\
$c(\AA)$ & 17.37094 \\
$\beta($ deg $)$ & 113.52900 \\
$V\left(\AA \AA^{3}\right)$ & 2407.54857 \\
$Z$ & 4 \\
$\rho_{\text {calcd. }\left(\mathrm{g} \cdot \mathrm{cm}^{-3}\right)}$ & 1.391 \\
$R_{1}$ & 0.0968 \\
$w R_{2}$ & 0.2830 \\
$F(000)$ & 1048.00 \\
Flack parameter & $0.03(3)$ \\
&
\end{tabular}




\section{Chemical Shifts of Fluorinated Azaindoline Amides and Related Compounds}

${ }^{1} \mathrm{H}$ NMR spectra were recorded on a Bruker AVANCE III HD 400 machine. Chemical shifts are reported in parts per million (ppm) downfield from tetramethylsilane and referenced to residual protium in the NMR solvent $\left(\mathrm{CDCl}_{3}: \delta=\right.$ $7.28 \mathrm{ppm}$ ); NMR data are reported as follows: chemical shift, multiplicity (d: doublet, $\mathrm{t}$ : triplet, q: quartet), and coupling constant $(\mathrm{Hz})$. The concentration of the individual samples were kept constant $\left(0.2 \mathrm{M}\right.$ in $\left.\mathrm{CDCl}_{3}\right)$; and the measurement temperatures were also constant $(300 \mathrm{~K})$ to assure comparability.

The synthesis of amides $\mathbf{1 a - 1 e}, \mathbf{1 a g}$, and $\mathbf{1 b g}$ is reported in section 4.1. Esters $\mathbf{1 a h}$ (Matrix Scientific Ltd.) and $\mathbf{1 b h}$ (Apollo Scientific Ltd.), as well as nitriles 1ai (Apollo Scientific Ltd.) and 1bi (SynQuest Laboratories, Inc.) were obtained from the indicated commercial suppliers.

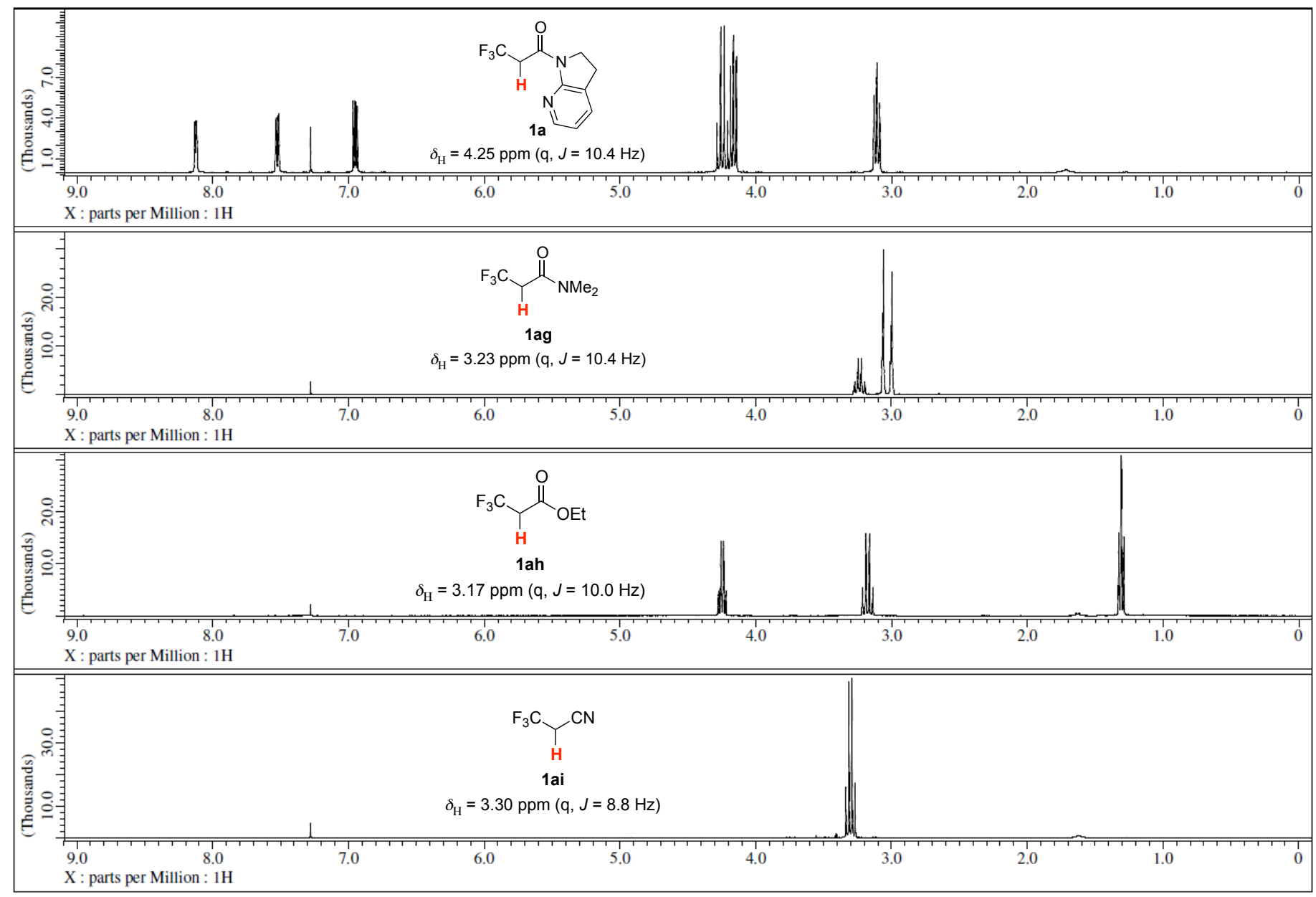

Chart S1. ${ }^{1} \mathrm{H}$ NMR spectra of amide 1a and related compounds. 

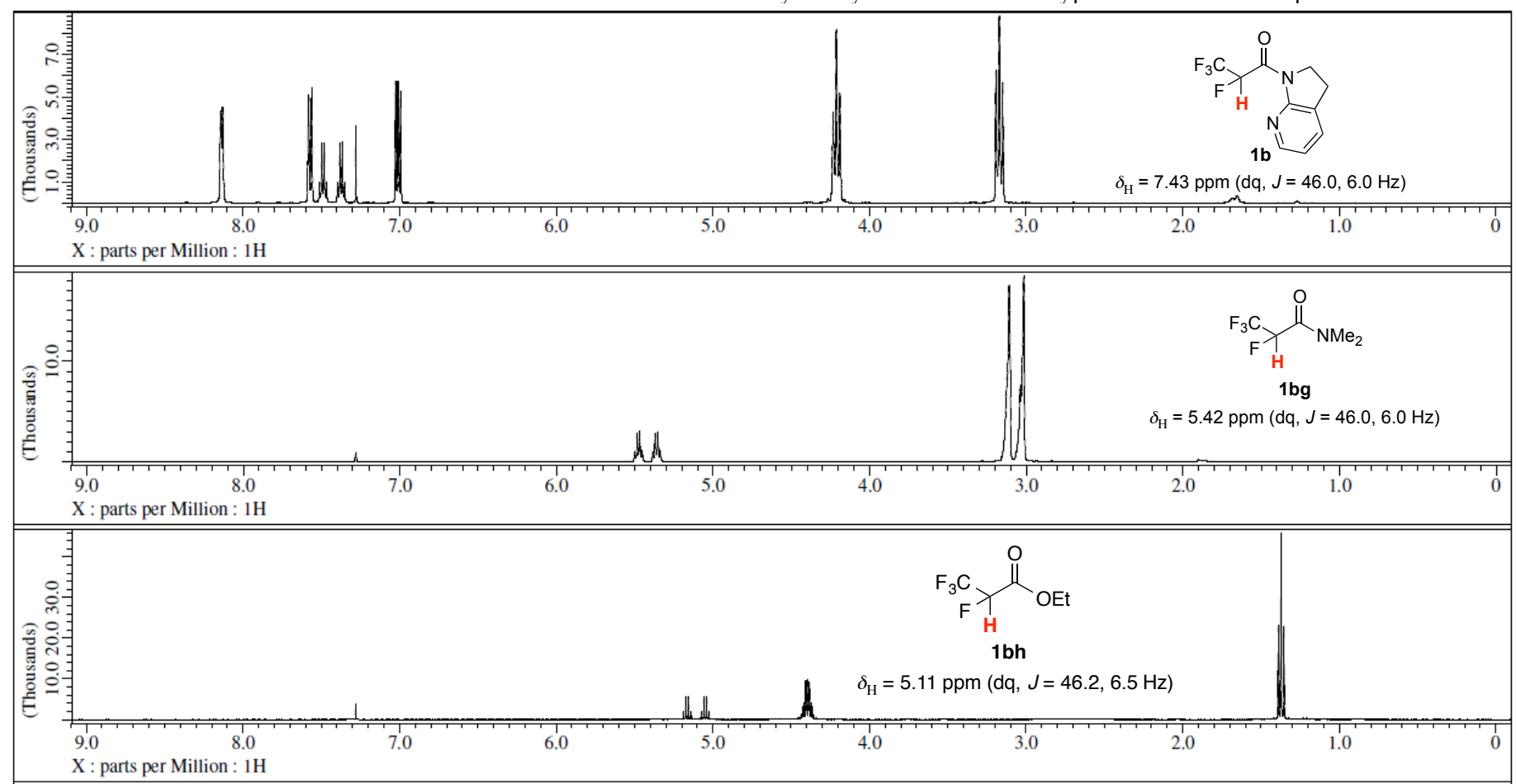

\begin{tabular}{|c|c|c|c|}
\hline \\
\hline
\end{tabular}

Chart S2. ${ }^{1} \mathrm{H}$ NMR spectra of amide $\mathbf{1 b}$ and related compounds. 


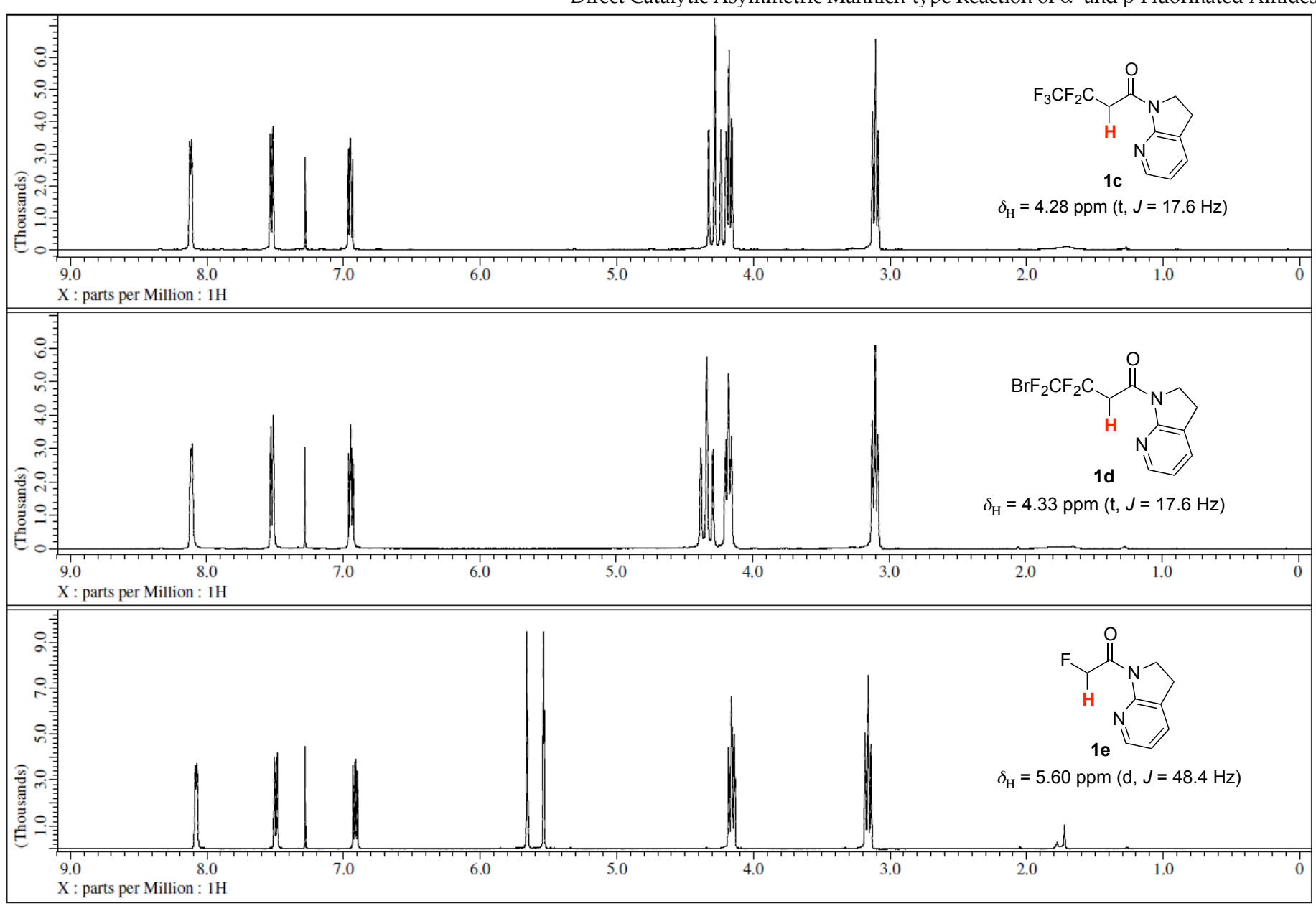

Chart S3. ${ }^{1} \mathrm{H}$ NMR spectra of amides $1 \mathrm{c}, \mathbf{1 d}$, and $\mathbf{1 e}$.

\section{Conformational Analysis of Amide}

Tertiary amides bearing an $\mathrm{N}$-alkyl and an $\mathrm{N}$-aryl group generally favor the conformation with the $\mathrm{N}$-aryl group at the opposite position to the carbonyl group. Amide 1a favors the E-conformation in solid state (see page S4). In the solution phase $\left(\mathrm{CDCl}_{3}\right), \mathrm{NOE}$ was not observed between the $\alpha$-protons and the protons at the indolinyl group, which implied 1a has E-conformation although it is not conclusive. Consistent ${ }^{1} \mathrm{H}$ NMR spectra of 1a at a range of temperatures suggest the predominant existence of only one conformer (likely the E-conformer) (Chart S4). 
(A) ${ }^{1} \mathrm{H}$ NMR of amide $1 \mathrm{a}$ in $\mathrm{CDCl}_{3}$ at $25^{\circ} \mathrm{C}$.

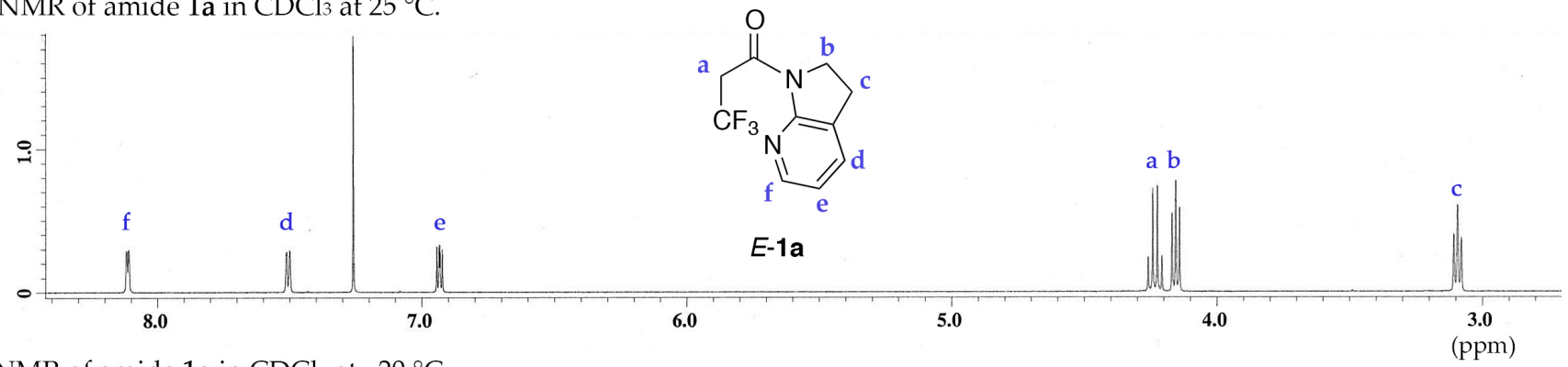

(B) ${ }^{1} \mathrm{H}$ NMR of amide $1 \mathrm{a}$ in $\mathrm{CDCl}_{3}$ at $-20{ }^{\circ} \mathrm{C}$.

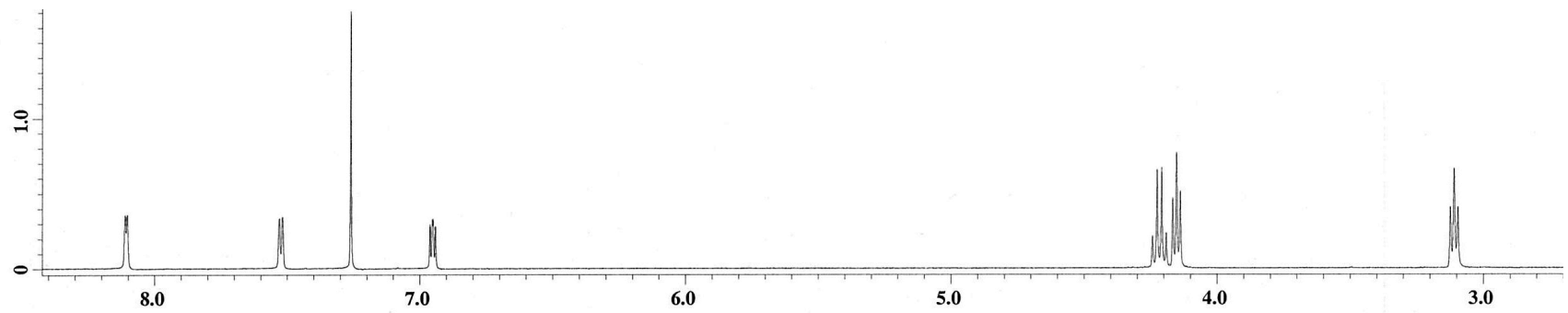

(C) ${ }^{1} \mathrm{H}$ NMR of amide $1 \mathrm{a}$ in $\mathrm{CDCl}_{3}$ at $-40{ }^{\circ} \mathrm{C}$.

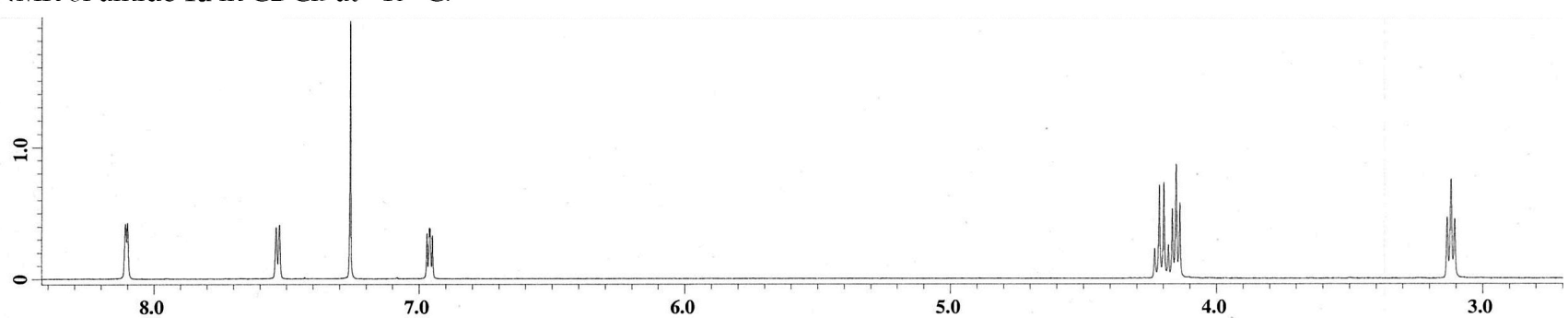

(D) ${ }^{1} \mathrm{H}$ NMR of amide $1 \mathrm{a}$ in $\mathrm{CDCl}_{3}$ at $-60^{\circ} \mathrm{C}$.

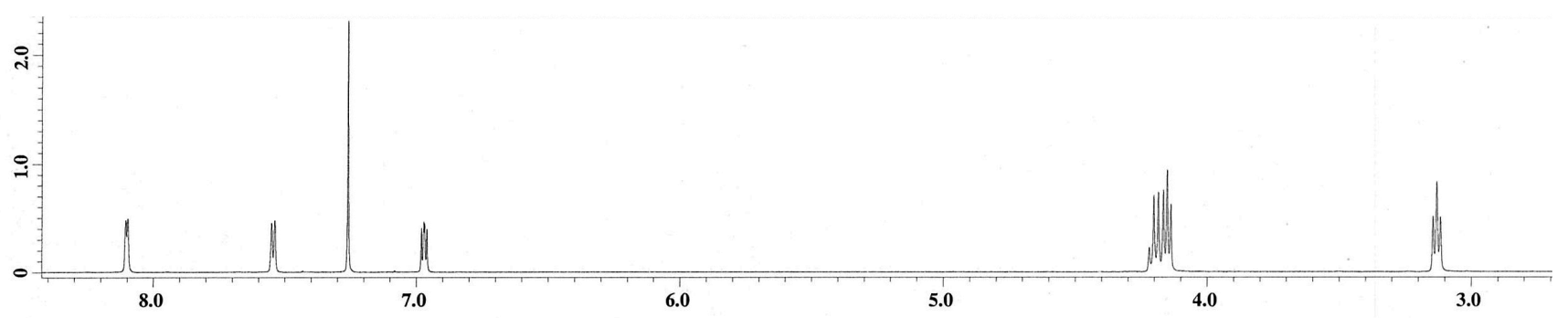

Chart S4. ${ }^{1} \mathrm{H}$ NMR spectra of amide $1 \mathrm{a}$ at variable temperatures.

${ }^{1} \mathrm{H}$ NMR spectra in the absence and presence of Brønsted acid showed clear differences (Chart S5). In the absence of acid, NOE was not observed between the $\alpha$-proton of $\mathbf{1 a}$ (protons a) and the indolinyl protons (protons $\mathbf{b}$ ) (Chart S5A,B). In sharp contrast, addition of TFA-d induced a clear change in the chemical shifts of 1a and NOE was observed between those protons, indicating that the Z-conformation was preferred upon coordination of the pyridyl group to deuterium (Chart S5C-E). Each peak was assigned based on COSY and HMQC analysis. A similar conformational change of an amide was reported in the literature. ${ }^{12}$ In the present case, chelation with the neighboring carbonyl group may contribute in addition for conformational change. 
(A) Differential NOE spectrum of amide $1 \mathrm{a}$ in $\mathrm{CD}_{2} \mathrm{Cl}_{2}$ (proton ' $\mathrm{a}$ ' was irradiated).
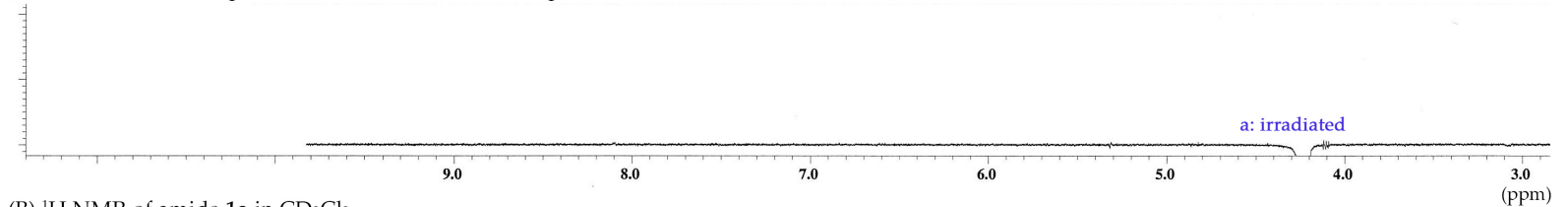

(B) ${ }^{1} \mathrm{H}$ NMR of amide $1 \mathrm{a}$ in $\mathrm{CD}_{2} \mathrm{Cl}_{2}$.

ppm)
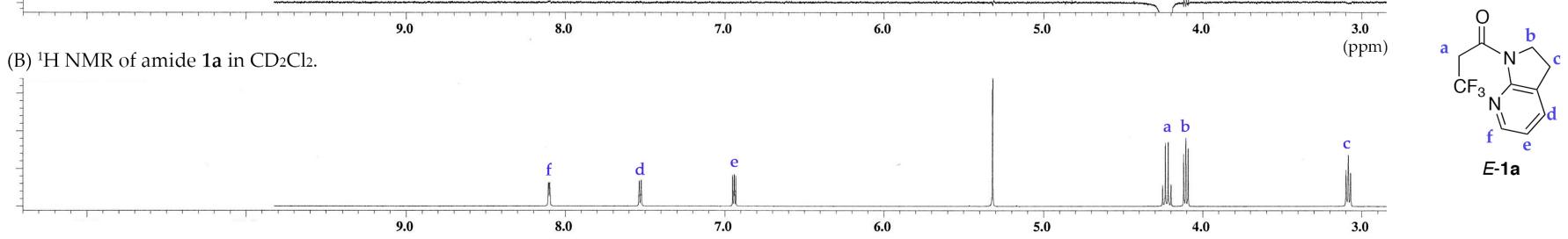

(C) ${ }^{1} \mathrm{H}$ NMR of amide $1 \mathrm{a}$ in $\mathrm{CD}_{2} \mathrm{Cl}_{2}$ in the presence of TFA- $d_{1}$.

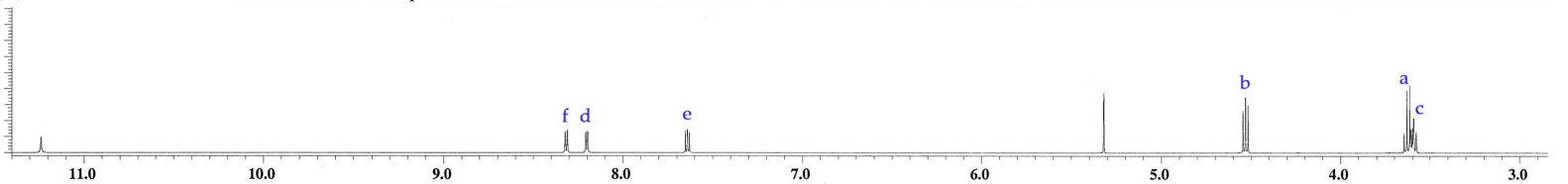

(D) Differential NOE spectrum of amide $1 \mathrm{a}$ in $\mathrm{CD}_{2} \mathrm{Cl}_{2}$ in the presence of TFA- $d_{1}$ (proton ' $b$ ' was irradiated).

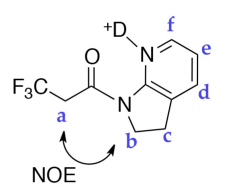

protonated $Z$-1a

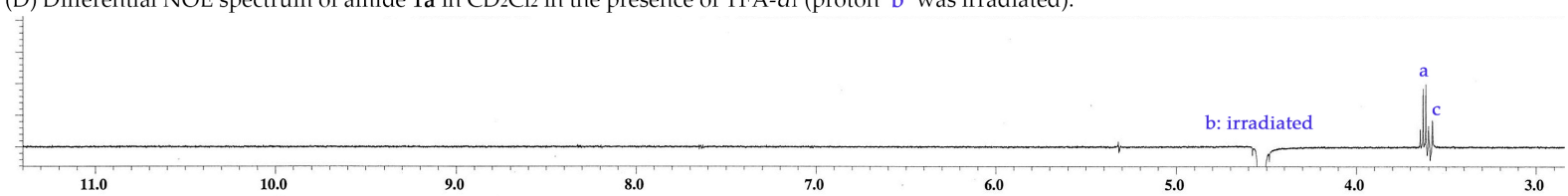

(E) Differential NOE spectrum of amide $1 \mathrm{a}$ in $\mathrm{CD}_{2} \mathrm{Cl}_{2}$ in the presence of TFA- $d_{1}$ (proton ' $a$ ' was irradiated).

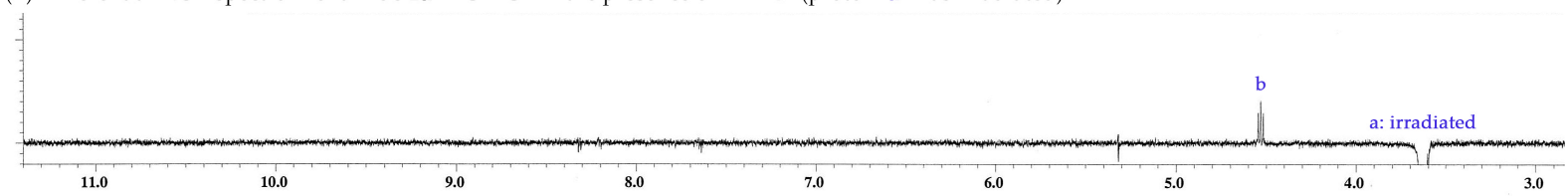

Chart S5. ${ }^{1} \mathrm{H}$ NMR and differential NOE spectra of amide 1a in the absence and presence of TFA.

A similar tendency was observed for $\alpha-\mathrm{F}-\alpha-\mathrm{CF}_{3}$ amide $\mathbf{1 b}$ (Chart S6). 


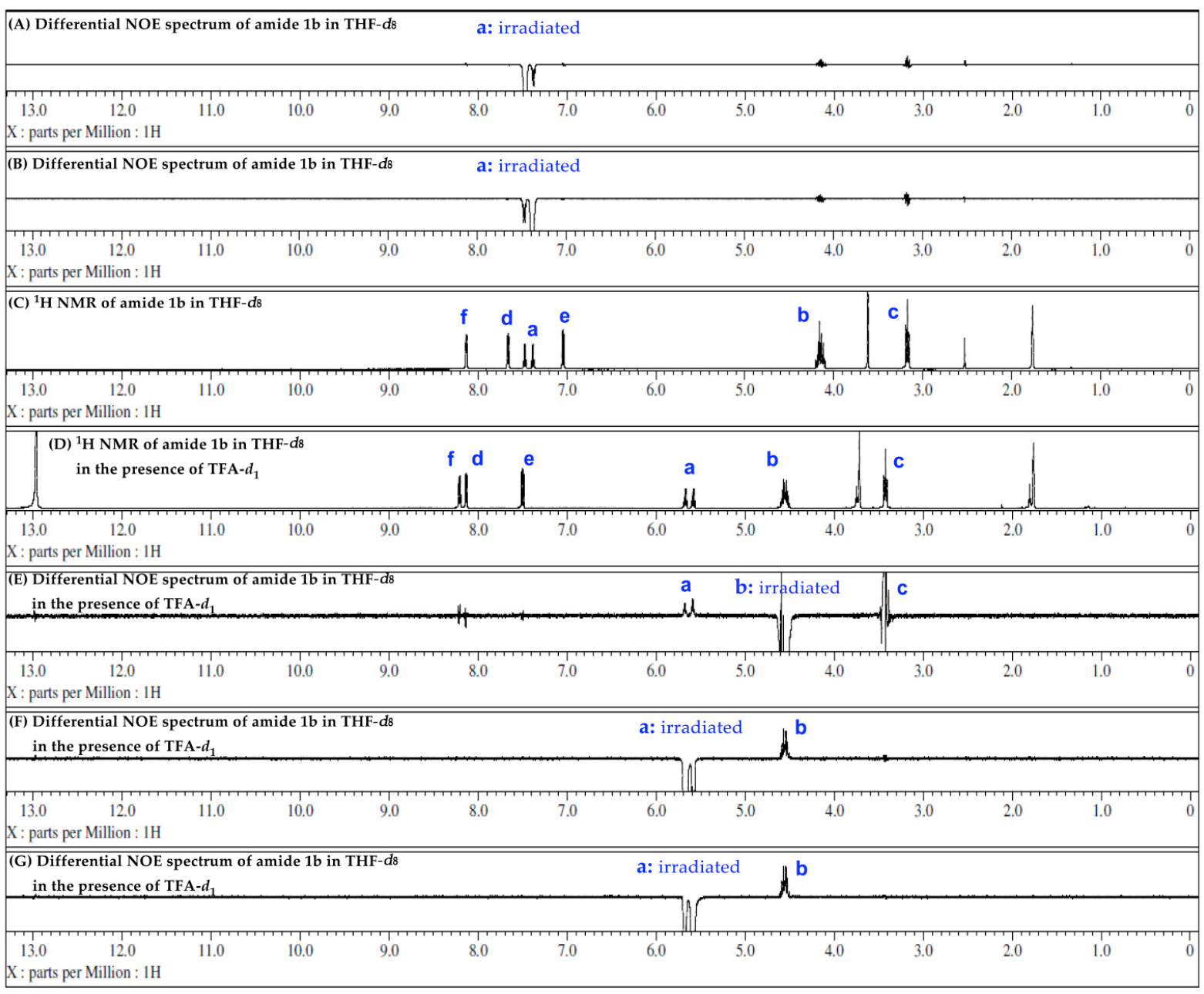

Chart S6. ${ }^{1} \mathrm{H}$ NMR and differential NOE spectra of $\mathbf{1 b}$ in the absence and presence of TFA

\section{Preparation and Characterization of $\mathrm{Cu}(\mathrm{I}) /$ rac-BINAP/1a Complex}

A flame-dried $20 \mathrm{~mL}$ test tube equipped with a magnetic stirring bar and 3-way glass stopcock was charged with $\left[\mathrm{Cu}\left(\mathrm{CH}_{3} \mathrm{CN}\right)_{4}\right] \mathrm{PF}_{6}(37.3 \mathrm{mg}, 0.10 \mathrm{mmol})$, rac-BINAP $(62.3 \mathrm{mg}, 0.10 \mathrm{mmol})$, and THF $(5.0 \mathrm{~mL})$. After the mixture was stirred for $30 \mathrm{~min}$ at room temperature, amide $1 \mathrm{a}(0.1 \mathrm{mmol}, 23.0 \mathrm{mg})$ was added to give a yellow solution, which was stirred for $2 \mathrm{~h}$ at room temperature. The mixture was transferred in glass vials to slowly grow crystals via vapor diffusion with $n$-hexane. Typically, the crystals were obtained in approximately $60 \%$ yield, and were collected by filtration washing with $n$-hexane.

Single-crystal X-ray data were collected on a Rigaku R-AXIS RAPID II imaging plate area detector with graphite-monochromated $\mathrm{Cu}-\mathrm{Ka}$ radiation. Data collection was conducted at $93 \mathrm{~K}$. All structures were solved by direct methods and refined by full matrix least-squares against $F^{2}$ with all reflections. All non-hydrogen atoms were refined anisotropically. All hydrogen atoms were placed in standard calculated positions, and were refined using the riding model. Refined structure and crystallographic parameters are summarized in Figure S9 and Table S9. CCDC 1032007 contains the supplementary crystallographic data for the complex. 


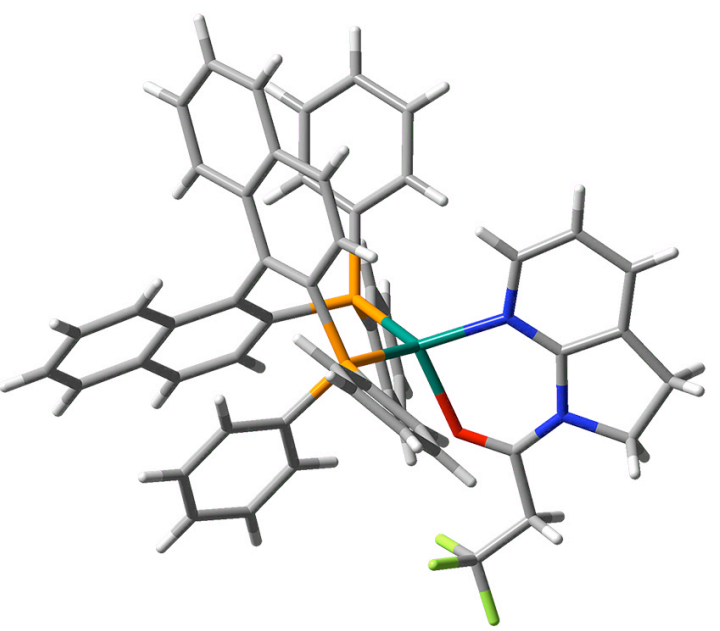

Figure S9. The structure of $\mathrm{Cu}(\mathrm{I}) / \mathrm{rac}-\mathrm{BINAP} / \mathbf{1 a}$ Complex. Dark green: copper, orange: phosphorous, lightgreen: fluorine, red: oxygen, blue: nitrogen, gray: carbon, white: hydrogen.
Table S9. Selected Crystallographic Data of $\mathrm{Cu}(\mathrm{I}) / r a c-B I N A P / \mathbf{1 a}$ Complex

\begin{tabular}{c|c}
\hline & $\mathrm{Cu}(\mathrm{I}) /$ rac-BINAP/1a Complex \\
\hline molecular formula & $\mathrm{C}_{59.88} \mathrm{H}_{52.33} \mathrm{CuF}_{9} \mathrm{~N}_{2} \mathrm{O}_{2.38} \mathrm{P}_{3}$ \\
formula weight & 1165.51 \\
crystal color, habit & yellow, chip \\
crystal system & monoclinic \\
space group & $P 21 / n$ \\
cell constants & \\
$a(\AA)$ & $18.2815(3)$ \\
$b(\AA)$ & $18.38753)$ \\
$c(\AA)$ & $33.1185(6)$ \\
$\alpha(\mathrm{deg})$ & 90.0000 \\
$\beta(\mathrm{deg})$ & $96.477(7)$ \\
$\gamma(\mathrm{deg})$ & 90.0000 \\
$V\left(\AA^{3}\right)$ & $1006.5(3)$ \\
$Z$ & 8 \\
$\rho_{\text {calcd }}\left(\mathrm{g} \mathrm{cm}{ }^{-3}\right)$ & 1.400 \\
$R_{1}$ & 0.0747 \\
$w R_{2}$ & 0.2349 \\
$F(000)$ & 4797.2 \\
\hline
\end{tabular}

The similar structure was expected in solution phase. Chart S7 depicts ${ }^{1} \mathrm{H}$ NMR spectra of $1 \mathrm{a}, \mathrm{Cu}(\mathrm{I}) / \mathrm{rac}-\mathrm{BINAP}$

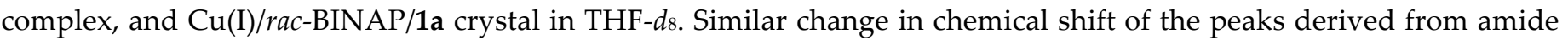
1a as observed in Chart S1 is indicative of the coordination of amide 1a to $\mathrm{Cu}(\mathrm{I}) / \mathrm{rac}$-BINAP complex. Each peak was assigned by COSY analysis. NOE analysis of the dissolved crystal confirmed the Z-conformation of the coordinated amide (Chart S8). For observation of clear NOE signals under concentrated conditions, the crystal was dissolved in $\mathrm{CD}_{2} \mathrm{Cl}_{2}$ (residual THF was also observed). 
(A) ${ }^{1} \mathrm{H}$ NMR spectrum of amide $1 \mathrm{a}$ in THF- $d 8$.

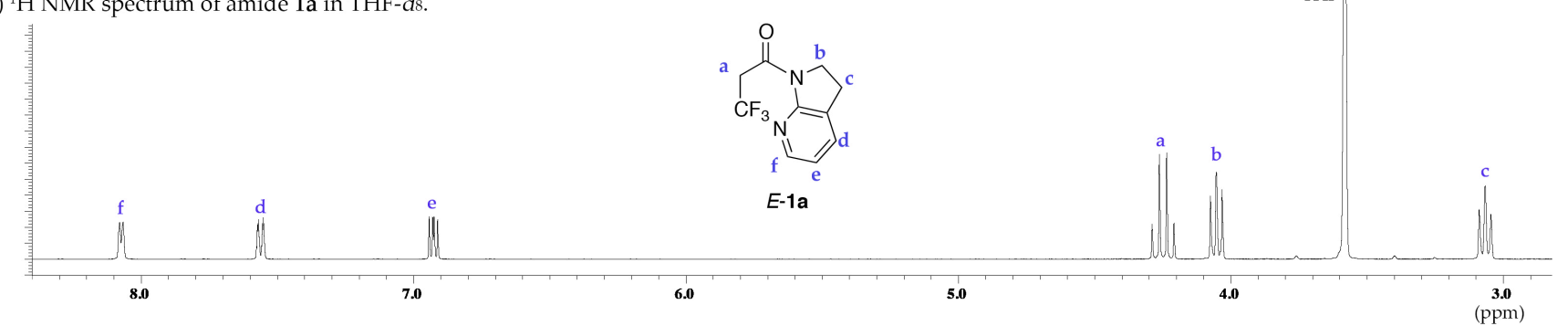

(B) ${ }^{1} \mathrm{H}$ NMR spectrum of the mixture of rac-BINAP and $\left[\mathrm{Cu}\left(\mathrm{CH}_{3} \mathrm{CN}\right) 4\right] \mathrm{PF} 6$ in THF- $d 8$.

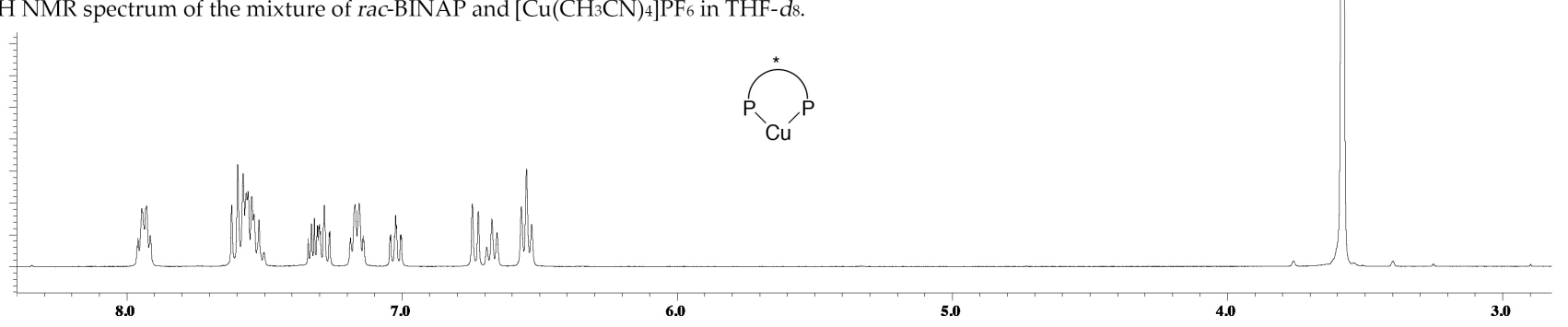

8.0

7.0

THF

(C) ${ }^{1} \mathrm{H}$ NMR spectrum of the crystal $(\mathrm{Cu}(\mathrm{I}) / \mathrm{rac}-\mathrm{BINAP} / 1 \mathrm{a})$ in THF-ds.

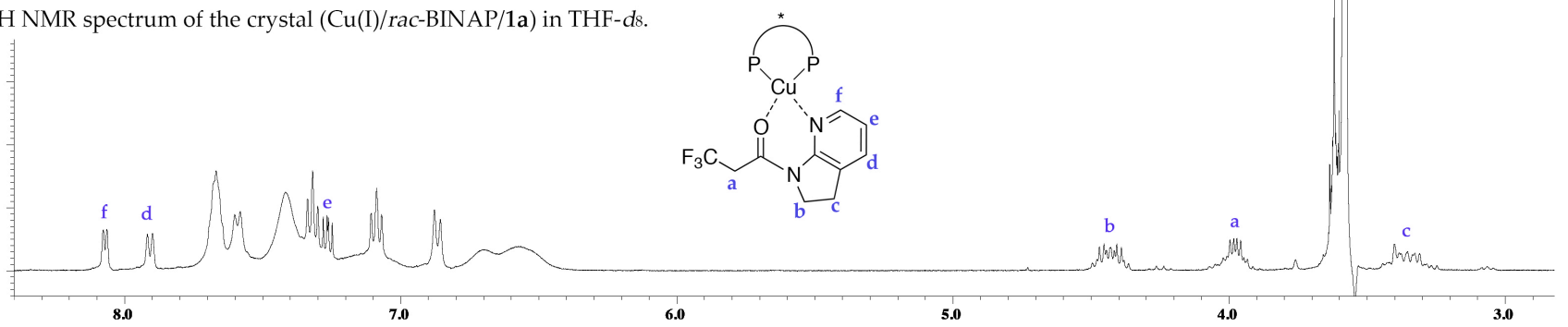

Chart S7. ${ }^{1} \mathrm{H}$ NMR of 1a (A), Cu(I)/rac-BINAP complex (B), and $\mathrm{Cu}(\mathrm{I}) /$ rac-BINAP/1a crystal (C).

(A) ${ }^{1} \mathrm{H}$ NMR spectrum of the crystal (Cu(I)/rac-BINAP/1a) in $\mathrm{CD}_{2} \mathrm{Cl}_{2}$.

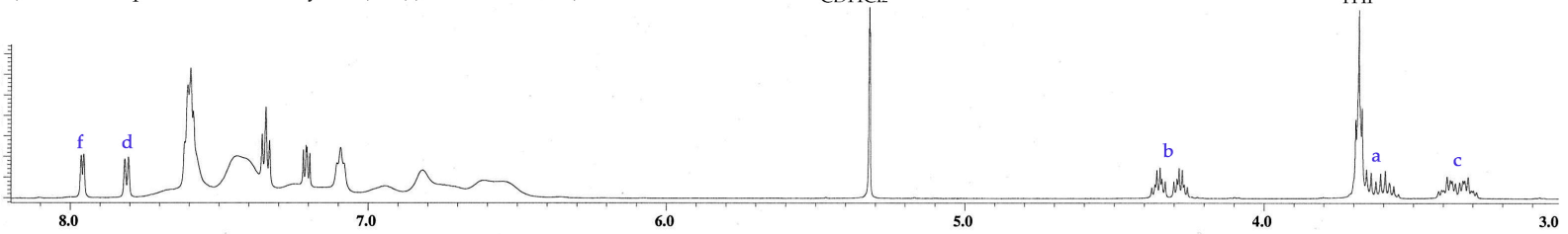

(B) Differential NOE spectrum of the crystal ( $\mathrm{Cu}(\mathrm{I}) /$ rac-BINAP/1a) in $\mathrm{CD}_{2} \mathrm{Cl}_{2}$ (proton ' a' was irradiated).
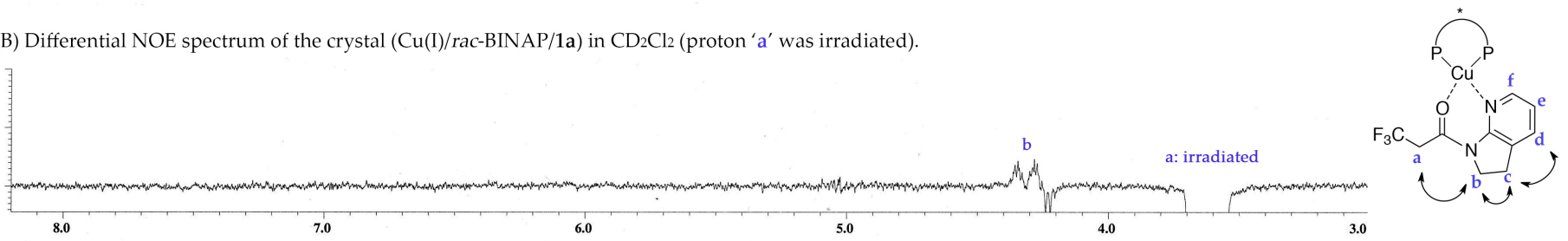

(C) Differential NOE spectrum of the crystal ( $\mathrm{Cu}(\mathrm{I}) / \mathrm{rac}-\mathrm{BINAP} / 1 \mathrm{a})$ in $\mathrm{CD}_{2} \mathrm{Cl}_{2}$ (proton ' $\mathrm{b}$ ' was irradiated).

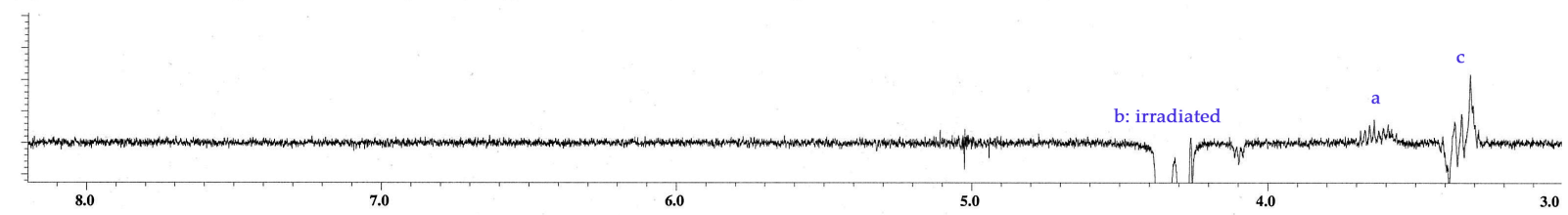

(D) Differential NOE spectrum of the crystal ( $\mathrm{Cu}(\mathrm{I}) / \mathrm{rac}-\mathrm{BINAP} / 1 \mathrm{a})$ in $\mathrm{CD}_{2} \mathrm{Cl}_{2}$ (proton ' $\mathrm{c}$ ' was irradiated).

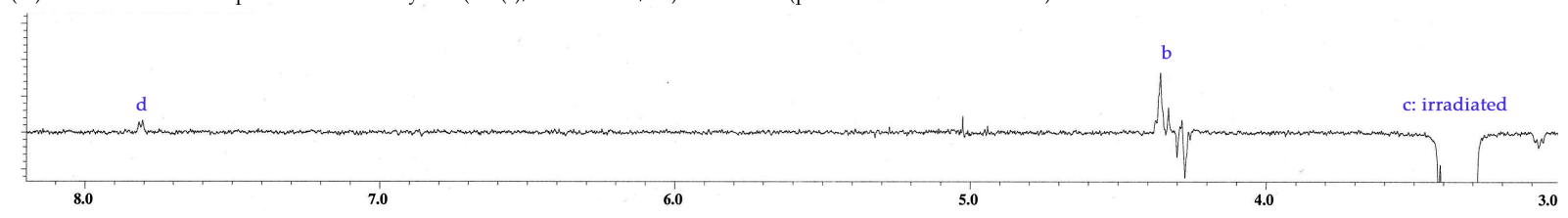

Chart S8. Differential NOE spectra of the $\mathrm{Cu}(\mathrm{I}) /$ rac-BINAP/1a crystal. 


\section{Separation of Amide (R)-1b and (S)-1b and Determination of the Absolute Configuration}

The enantiomers of the racemic amide rac-1b were separated using a CHIRALPAK IC $(\phi=2.0 \mathrm{~cm} \times 25 \mathrm{~cm})$ column with a solvent mixture of 2-propanol $/ n$-hexane $=1 / 2$ and a flow rate of $20.0 \mathrm{~mL} / \mathrm{min}$. The detection was performed at a wavelenght of $254 \mathrm{~nm}$. The retention times (tr) of the enantiomers were: $7.4 \mathrm{~min}((R)$-enantiomer) and $9.5 \mathrm{~min}$ ((S)-enantiomer). The racemic amide was separated in batches of $50 \mathrm{mg}$ (injected as a solution in $1.0 \mathrm{~mL} \mathrm{CHCl}_{3}$ ). The absolute configuration of the (S)-enantiomer was determined by X-ray analysis of a suitable single-crystal. Analytical data for the two enantiomers are shown below:

\section{(R)-Enantiomer:}

$[\alpha]_{D}^{26}=-15.0\left(c=1.00, \mathrm{CHCl}_{3},>99 \%\right.$ ee sample); HPLC analysis (CHIRALPAK IC $(\phi=0.46 \mathrm{~cm} \times 25 \mathrm{~cm})$, 2-propanol $/ n$-hexane $=1 / 2$, flow rate $=1.0 \mathrm{~mL} / \mathrm{min}$, detection at $254 \mathrm{~nm}, \mathrm{tR}=7.4 \mathrm{~min}$ :
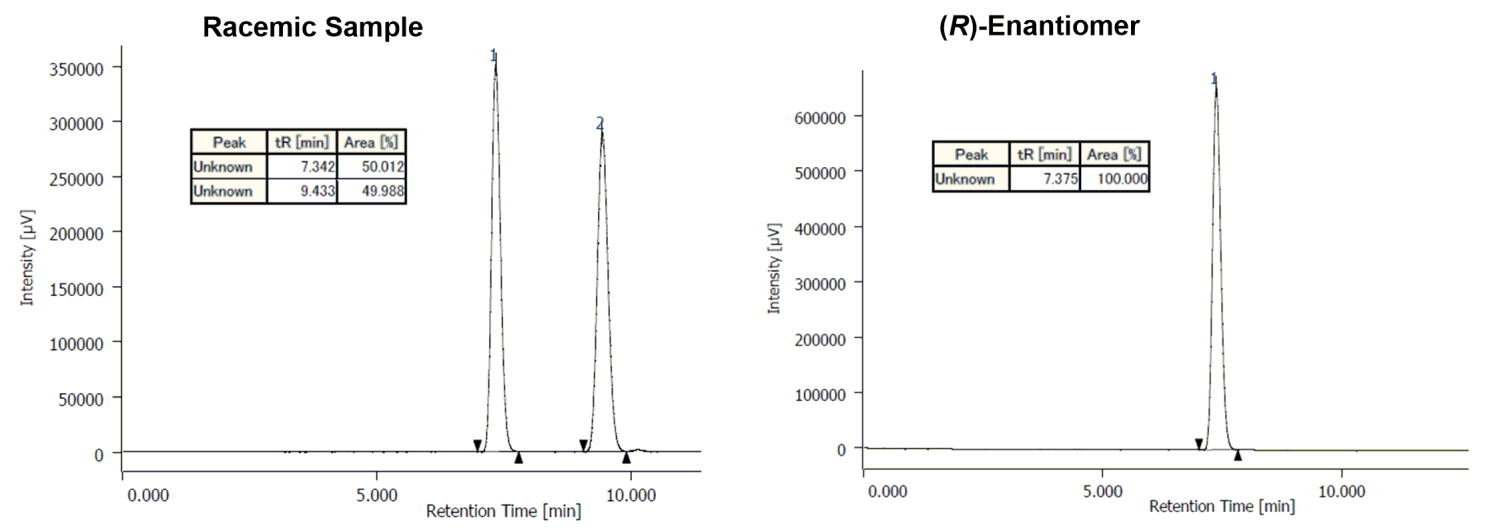

(S)-Enantiomer:

$[\alpha]_{D}^{26}=+13.5\left(c=1.00, \mathrm{CHCl}_{3},>99 \%\right.$ ee sample); HPLC analysis (CHIRALPAK IC $(\phi=0.46 \mathrm{~cm} \times 25 \mathrm{~cm})$, 2-propanol $/ n$-hexane $=1 / 2$, flow rate $=1.0 \mathrm{~mL} / \mathrm{min}$, detection at $254 \mathrm{~nm}, \mathrm{t} R=9.5 \mathrm{~min}$ :
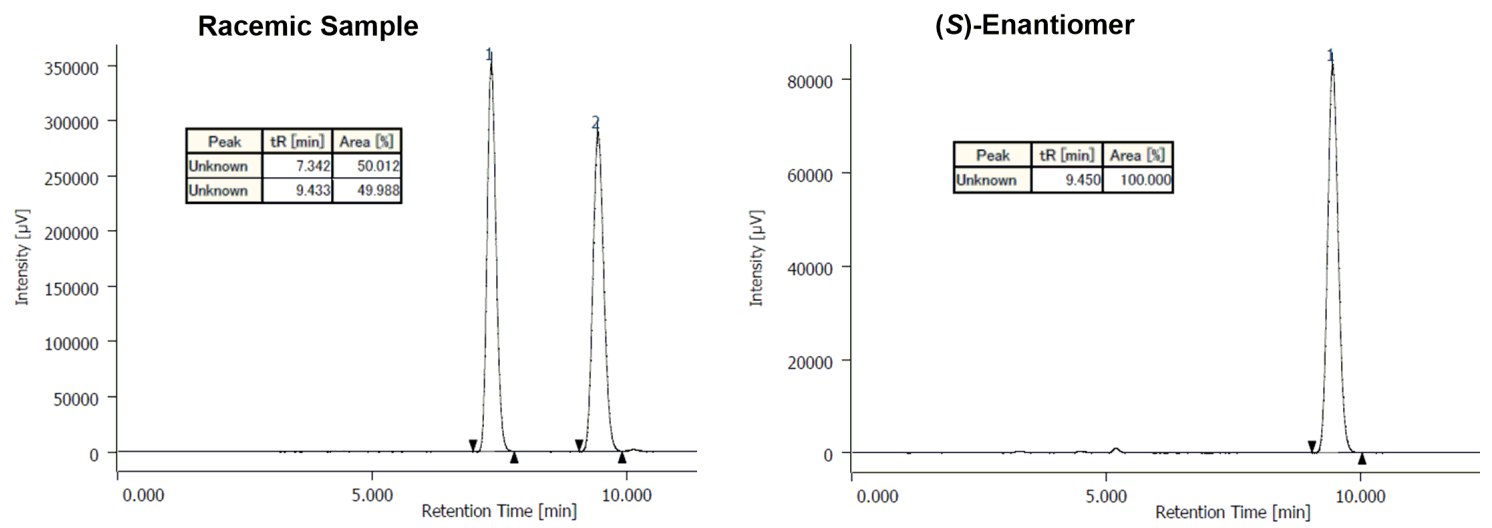

In order to determine the absolute configuration of the amide (S)-1 $\mathbf{b}$, a single-crystal was grown by leaving a concentrated solution of (S)-1b in $n$-hexane/diethylether at room temperature. Single-crystal X-ray data were collected on a Rigaku R-AXIS RAPID II imaging plate area detector with graphite-monochromated $\mathrm{Cu}-\mathrm{K} \alpha$ radiation. Data collection was conducted at $93 \mathrm{~K}$. All structures were solved by direct methods and refined by full matrix least-squares against $F^{2}$ with all reflections. All non-hydrogen atoms were refined anisotropically. All hydrogen atoms were placed 

positions, and were refined using the riding model. Refined structure and crystallographic

pa1 rized in Figure S10 and Table S10. CCDC 1430612 contains the supplementary crystallographic

data ive (u)

The abšlofue canfiguration of $(S)-\mathbf{1 b}$ was determined to be as depicted below by Flack parameter. ${ }^{11}$<smiles>CC(F)CC1N[Si]c2cccnc21</smiles>

Figure S10. The structure of $(S)-\mathbf{1 b}$ in the solid state. Color code: Light green: fluorine; red: oxygen; blue: nitrogen; gray: carbon; white: hydrogen.
Table S10. Selected Crystallographic Data of (S)-1b

\begin{tabular}{c|c}
\hline & $(S)-\mathbf{1 b}$ \\
\hline molecular formula & $\mathrm{C}_{10} \mathrm{H}_{8} \mathrm{~F}_{4} \mathrm{~N}_{2} \mathrm{O}$ \\
formula weight & 248.18 \\
crystal color, habit & colorless, prism \\
crystal system & orthorhombic \\
space group & $P 212121$ \\
cell constants: & \\
$a(\AA)$ & $4.95050(11)$ \\
$b(\AA)$ & $9.1116(2)$ \\
$c(\AA)$ & $22.1601(5)$ \\
$V\left(\AA^{3}\right)$ & $999.57(4)$ \\
$Z$ & 4 \\
$\rho_{\text {calcd. }}(\mathrm{g} \cdot \mathrm{cm}-3)$ & 1.649 \\
$R_{1}$ & 0.0509 \\
$w R_{2}$ & 0.1027 \\
F(000) & 504.00 \\
Flack parameter & $0.19(17)$ \\
\end{tabular}




\section{HOESY Analysis of $\mathrm{Cu}(\mathrm{I}) /(R)-x y l-B I N A P /(S)-1 b$ Complex}

A ${ }^{1} \mathrm{H}-{ }^{19} \mathrm{~F}$ HOESY experiment was performed on a $1 / 1$ mixture of $\mathrm{Cu}(\mathrm{I}) /(R)-\mathrm{L} 6$ complex and $\alpha-\mathrm{F}-\alpha-\mathrm{CF}_{3}$ amide $(S)-1 \mathbf{b}$ (Chart S9). Signal A indicated the Z-conformer of the amide $\mathbf{1 b}$ upon complexation with the $\mathrm{Cu}(\mathrm{I})$ complex. Unexpected signals B-D were attributed to the fast equilibrium between the free amide and the complex. The required mixing time for HOESY experiment was slower than the association/dissociation kinetics of the complex.<smiles>CC1Cc2cccnc2N1C(=O)C(F)(F)C(F)(F)F</smiles>

$(S)-1 b$

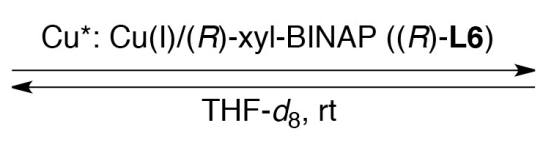

${ }^{1} \mathrm{H}-19 \mathrm{~F}$ HOESY

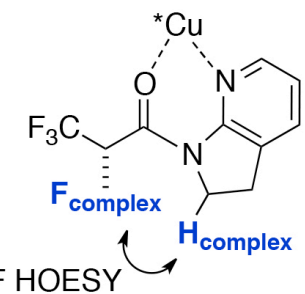

$(S)-1 \mathrm{~b} / \mathrm{Cu}(\mathrm{I}) /(R)-\mathrm{xyl}$-BINAP $((R)-\mathrm{L} 6)$

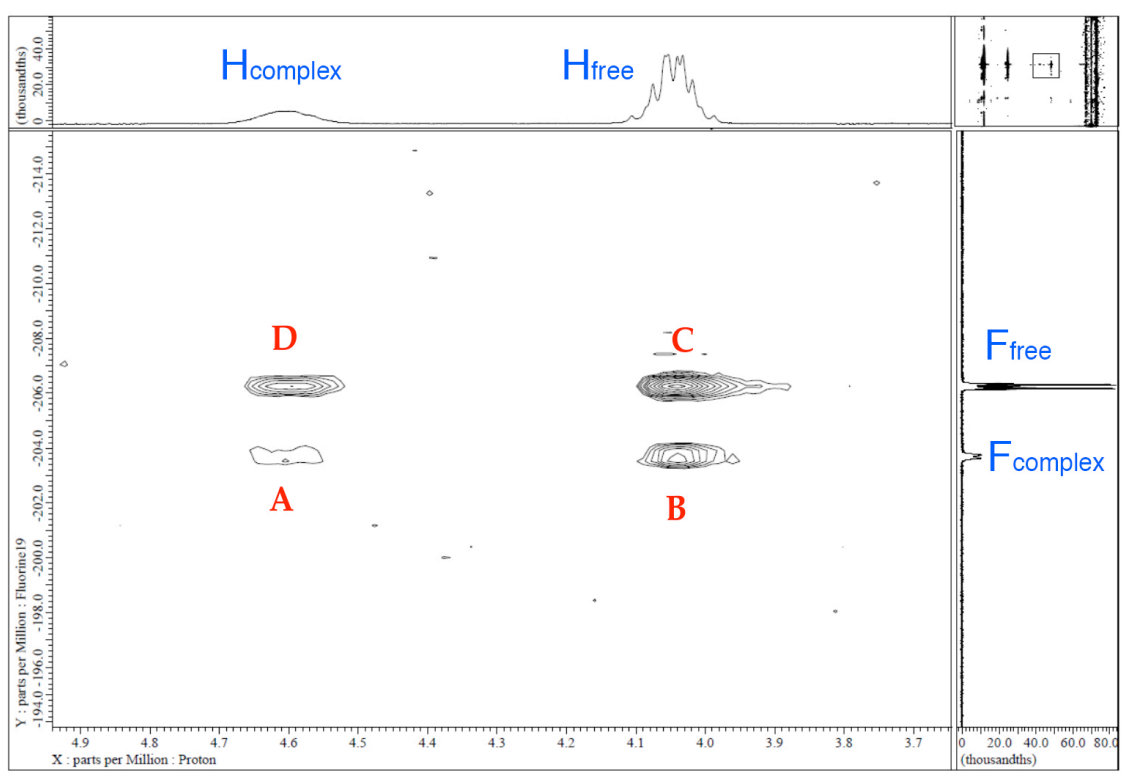

Chart S9. ${ }^{1} \mathrm{H}-19 \mathrm{~F}$ HOESY Spectrum of a 1/1 mixture of $\mathrm{Cu}(\mathrm{I}) /(R)-\mathrm{L} 6$ complex and $(S)-\mathbf{1 b}$.

\section{Ligand Screening for the Mannich-type Reaction of $\alpha$-F Amide 1e}

The ligand screening for the optimization of the Mannich-type reaction of amide 1e is shown in Table S11. Procedure $\mathrm{D}$ (see section 4.7) was followed using the different ligands shown. $\left(R, R_{P}\right)$-Cy-Taniaphos was identified as the best ligand affording product $\mathbf{1 5}$ in good diastereoselectivity and excellent enationselectivity. In order to improve the conversion and selectivities of this reaction, different solvents, concentrations, reaction temperatures and times were examined (not shown). However, the result presented in entry 25 could not be improved. 
Table S11. Ligand Screening for the Mannich-type reaction of $\alpha$-F amide 1e

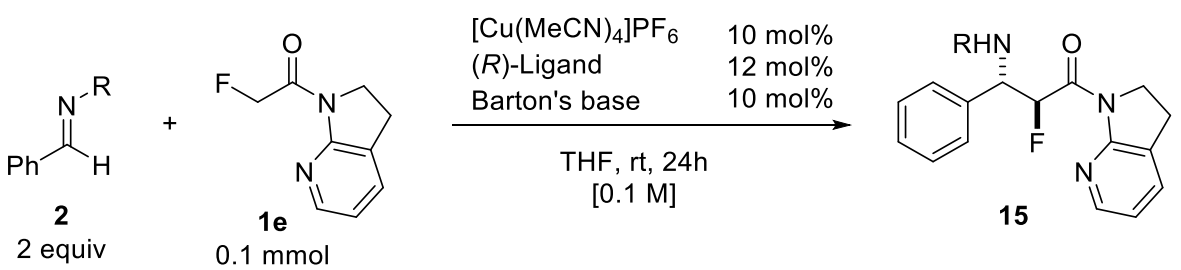

\begin{tabular}{|c|c|c|c|c|c|}
\hline Entry & $\mathbf{R}$ & $(R)$-Ligand & Conversion $[\%]^{a}$ & $\operatorname{dr}(a n t i / s y n)^{b}$ & ee $(a n t i, s y n)^{b}$ \\
\hline 1 & $\operatorname{Boc}(2 a)$ & tol-BINAP & 83 & $0.6 / 1$ & 21,22 \\
\hline 2 & $\operatorname{Boc}(2 a)$ & SEGPHOS & 83 & $0.6 / 1$ & 37,40 \\
\hline 3 & $\operatorname{Boc}(2 a)$ & Ph-BPE & 78 & $0.7 / 1$ & $-72,-85$ \\
\hline 4 & $\operatorname{Boc}(2 \mathbf{a})$ & Josiphos & 55 & $0.8 / 1$ & 39,54 \\
\hline 5 & $\operatorname{Boc}(\mathbf{2 a})$ & DTBM-Garphos & 40 & $1.1 / 1$ & 67,16 \\
\hline 6 & $\operatorname{Boc}(2 a)$ & DIPA-MeO-BIPHEP & 44 & $0.7 / 1$ & 35,4 \\
\hline 7 & $\operatorname{Boc}(2 a)$ & $(R, R P)$-Taniaphos-1 & 82 & $2.6 / 1$ & $-27,11$ \\
\hline 8 & $\operatorname{Boc}(\mathbf{2 a})$ & $(R, R P)$-Taniaphos-2 & 70 & $1.2 / 1$ & $-60,-16$ \\
\hline 9 & $\mathrm{Cbz}(2 \mathbf{b})$ & tol-BINAP & 43 & $0.9 / 1$ & $12, \mathrm{rac}$ \\
\hline 10 & $\mathrm{Cbz}(\mathbf{2 b})$ & xyl-BINAP & 72 & $0.7 / 1$ & $-13,4$ \\
\hline 11 & $\mathrm{Cbz}(\mathbf{2 b})$ & SEGPHOS & 50 & $0.8 / 1$ & $-23,14$ \\
\hline 12 & $\mathrm{Cbz}(2 \mathbf{b})$ & DTBM-SEGPHOS & 60 & $1.0 / 1$ & $-64,20$ \\
\hline 13 & $\mathrm{Cbz}(2 \mathbf{b})$ & Ph-BPE & 83 & $1.6 / 1$ & $79,-85$ \\
\hline 14 & $\mathrm{Cbz}(2 \mathbf{b})$ & Walphos-2 & 70 & $0.5 / 1$ & $-35,60$ \\
\hline 15 & $\mathrm{Cbz}(\mathbf{2 b})$ & Josiphos-2 & 72 & $0.5 / 1$ & $-33,59$ \\
\hline 16 & $\mathrm{Cbz}(2 \mathrm{~b})$ & Ph-Garphos & 67 & $0.9 / 1$ & 18,11 \\
\hline 17 & $\mathrm{Cbz}(\mathbf{2 b})$ & DTBM-Garphos & 70 & $1.3 / 1$ & $70, \mathrm{rac}$ \\
\hline 18 & $\mathrm{Cbz}(\mathbf{2 b})$ & 3,4,5-MeO-MeO-BIPHEP & 66 & $0.6 / 1$ & $-79,88$ \\
\hline 19 & $\mathrm{Cbz}(\mathbf{2 b})$ & DIPA-MeO-BIPHEP & 54 & $0.8 / 1$ & 50,3 \\
\hline 20 & $\mathrm{Cbz}(\mathbf{2 b})$ & DTBM-MeO-BIPHEP & 71 & $1.1 / 1$ & 66,5 \\
\hline 21 & $\mathrm{Cbz}(2 \mathbf{b})$ & $\left(R, R_{P}\right)$-Ph-Taniaphos & 80 & $8.1 / 1$ & $70,-11$ \\
\hline $22^{c, d}$ & $\mathrm{Cbz}(2 \mathrm{~b})$ & $\left(R, R_{P}\right)$-Ph-Taniaphos & 70 & $15.7 / 1$ & 78, nd \\
\hline 23 & $\mathrm{Cbz}(\mathbf{2 b})$ & $\left(R, R_{P}\right)-\mathrm{Cy}$-Taniaphos & 90 & $8.1 / 1$ & $87,-11$ \\
\hline $24^{c, e}$ & $\mathrm{Cbz}(2 \mathbf{b})$ & $(R, R P)-C y-T a n i a p h o s$ & 90 & $10.1 / 1$ & $91, \mathrm{nd}$ \\
\hline $25^{c, d, f}$ & $\mathrm{Cbz}(2 \mathrm{~b})$ & $\left(R, R_{P}\right)$-Cy-Taniaphos & 91 & $8.9 / 1$ & $91, \mathrm{nd}$ \\
\hline
\end{tabular}

${ }^{a}$ Determined by ${ }^{1} \mathrm{H}$ NMR. ${ }^{b}$ Determined by chiral stationary HPLC. ${ }^{c}$ At $0{ }^{\circ} \mathrm{C} .{ }^{d}$ After $48 \mathrm{~h} .{ }^{e}$ After 72 h. $f 0.2 \mathrm{M}$ on 1 e.

\section{Acute Toxicity of $\alpha-F$ Amide 1e}

Due to the severe toxicity of monofluoroacetic acid (LD50 $=0.1 \mathrm{mg} / \mathrm{kg}$ ), the acute toxicity of structurally related amide 1e was investigated using ICR female mice. No fatality was observed up to $12.5 \mathrm{mg} / \mathrm{kg}$ by intravenous administration, but mice died at $25 \mathrm{mg} / \mathrm{kg}$ and more. Although the toxicity of $\mathbf{1 e}$ was significantly lower compared to monofluoroacetic acid, appropriate safety measures (fume hood, mask, gloves) must be followed to avoid direct contact. 
(1) (a) Pearson, S. E.; Nandan, S. Synthesis 2005, 2503; (b) Darout, E. et al. WO2010/128414 A1, 2010.

(2) Yin, L.; Brewitz, L.; Kumagai, N.; Shibasaki, M. J. Am. Chem. Soc. 2014, 136, 17958.

(3) (a) Pederson, B. F.; Pederson, B. Tetrahedron Lett. 1965, 2995; (b) Nanjan, M. J.; Kannappan, V.; Ganesan, R. Indian J. Chem. 1979, 18B, 461; (c) Itai, A.; Toriumi, Y.; Tomioka, N.; Kagechika, H.; Azumaya, I.; Shudo, K. Tetrahedron Lett. 1989, 30, 6177; (d) Saito, S.; Toriumi, Y.; Tomioka, N.; Itai, A. J. Org. Chem. 1995, 60, 4715; (e) Bartuschat, A. L.; Wicht, K.; Heinrich, M. R. Angew. Chem., Int. Ed. 2015, 54, 10294.

(4) (a) Weidner, K.; Kumagai, N.; Shibasaki, M. Angew. Chem., Int. Ed. 2014, 53, 6150; (b) Weidner, K.; Sun, Z.; Kumagai, N.; Shibasaki, M. Angew. Chem., Int. Ed. 2015, 54, 6236.

(5) Aqad, E.; Li, M.; Xu, C.-B. EP2372455 A2, 2011.

(6) Mantani, T.; Shiomi, K.; Konno, T.; Ishihara, T.; Yamanaka, H. J. Org. Chem. 2001, 66, 3442.

(7) (a) Kanazawa, A. M.; Denis, J.-N.; Greene, A. E. J. Org. Chem. 1994, 59, 1238; (b) Love, B. E.; Raje, P. S.; Williams II, T. C. Synlett 1994, 493; (c) Wenzel, A. G.; Jacobsen, E. N. J. Am. Chem. Soc. 2002, 124, 12964; (d) Mbofana, C. T.; Miller, S. J. J. Am. Chem. Soc. 2014, 136, 3285.

(8) (a) Song, J.; Wang, Y.; Deng, L. J. Am. Chem. Soc. 2006, 128, 6048; (b) Handa, S.; Gnanadesikan, V.; Matsunaga, S.; Shibasaki, M. J. Am. Chem. Soc. 2010, 132, 4925; (c) Neuvonen, A. J.; Pihko, P. M. Org. Lett. 2014, 16, 5152.

(9) (a) Tillman, A. L.; Ye, J.; Dixon, D. J. Chem. Commun. 2006, 1191; (b) Mbofana, C. T.; Miller, S. J. J. Am. Chem. Soc. $2014,136,3285$.

(10) The occurance of rotamers is well documented for similar compounds, see for example: Peddie, V.; Abell, A. D. Helv. Chim. Acta 2012, 95, 2460.

(11) Flack, H. D. Acta Cryst. 1983, A39, 876.

(12) Yamasaki, R.; Tanatani, A.; Azumaya, I.; Saito, S.; Yamaguchi, K.; Kagechika, H. Org. Lett. 2003, 5, 1265.

\section{4. ${ }^{1} \mathrm{H},{ }^{13} \mathrm{C}$, and ${ }^{19} \mathrm{~F}$ NMR Spectra of the New Compounds (see separate file)}

The ${ }^{1} \mathrm{H},{ }^{13} \mathrm{C}$, and ${ }^{19} \mathrm{~F}$ NMR spectra of the new compounds are shown in a separated pdf file of this supporting information (p. 74-240) and can be downloaded free of charge from the ACS/JACS internet page.

The ${ }^{1} \mathrm{H},{ }^{13} \mathrm{C}$, and ${ }^{19} \mathrm{~F}$ NMR spectra of the compounds which have already been published in a previous communication of this work (1ab-1ad, 2a, 2c-2h, 2k, 21, 2n-2t, 4, 5) can be downloaded free of charge from the ACS/JACS internet page under the following link:

http://pubs.acs.org/doi/suppl/10.1021/ja511458k 